LBL -32218

DE92 0.16928

Signal and Noise Analysis of a-Si:H Radiation Detector-Amplifier System

\author{
Gyuseong Cho \\ Ph.D. Thesis \\ Department of Nuclear Engineering \\ University of California \\ and \\ Physics Division \\ Lawrence Berkeley Laboratory \\ University of California \\ Berkeley, CA 94720
}

April 1992

\title{
MASTER
}

This work was supported by the Director, Office of Energy Research, Office of High Energy and Nuclear Physics, Division of High Energy Physics of the U.S. Department of Energy under Contract No. DE-AC03-76SF00098. 


\title{
Signal and Noise Analysis of \\ a-Si:H Radiation Detector-Amplifier System
}

\author{
by \\ Gyuseong Cho
}

\begin{abstract}
Hydrogenated amorphous silicon (a-Si:H) has potential advantages in making radiation detectors for many applications because of its deposition capability on a large-area substrate and its high radiation resistance. Position-sensitive radiation detectors can be made out of a 1-d strip or a 2-d pixel array of a-Si:H pin diodes. In addition, signal processing electronics can be made by thin -film transistors (amorphous silicon or polysilicon TFTs) on the same substrate.

The calculated radiation signal, based on a simple charge collection model agreed well with results from various wave-length light sources and $1 \mathrm{MeV}$ beta particles on sample diodes. The total noise of the detection system was analyzed into (a) shot noise and (b) $1 / f$ noise from a detector diode, and (c) thermal noise and (d) $1 / f$ noise from the frontend TFT of a charge-sensirive preamplifier. The effechive noise charge calculated by convoluting these noise power spectra with the transfer function of a CR-RC shaping amplifier showed a good agreement with the direct measurements of noise charge. The derived equations of signal and noise charge can be used to design an a-Si:H pixel detectoramplifier system optimally.

Signals from a pixel can be readout using switching TFTs, or diodes. Prototype tests of a double-diode readout scheme showed that the storage time and the readout time are limited by the resistances of the reverse-biased pixel diode and the forward-biased switching diodes respectively. A prototype charge-sensitive amplifier was made using


poly-Si TFTs to test the feasibility of making pixel-level amplifiers which would be required in small-signal detection. The measured overall gain-bandwidth product was $\sim 400$ $\mathrm{MHz}$ and the noise charge was $\sim 1000$ electrons at a $1 \mu \mathrm{sec}$ shaping time. When the amplifier is connected to a pixel detector of capacitance $0.2 \mathrm{pF}$, it would give a charge-tovoltage gain of $\sim 0.02 \mathrm{mV} / \mathrm{electron}$ with a pulse rise time less than $100 \mathrm{nsec}$ and a dynamic range of $48 \mathrm{~dB}$. 


\section{Signal and Noise Analysis of a-Si:H Radiation Detector-Amplifier System}

Copyright (c) 1991

by

Gyuseong Cho

The United States Department of Energy has the right to use this thesis for any purpose whatsoever including the right to reproduce all or any part thereof. 
To my family 
Table of Contents

List of Figures

List of Tables

List of Symbols -

Acknowledgement -

Chapter 1 Introduction

Chapter 2 Background

2.1 Position-Sensitive Radiation Detectors $\quad$.................................. 3

2.1.1 Silicon Pixel Detectors

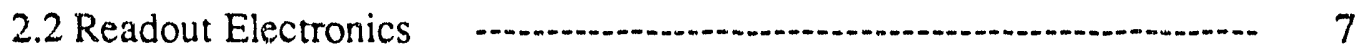

2.2.1 Readout Schemes for 1-D Detector Ariay

2.2.2 Readout Schemes for 2-D Detector Array

2.3 Hydrogenated Amorphous Silicon (a-Si:H) …_..................... 12

2.3.1 Production method

2.3.2 Basic Properties

2.4 a-Si:H Pixel Detector Structures

19

2.4.1 Thin Detector Coupled to Scintillating CsI(Tl) Layer

2.4.2 Thick Detector for Direct Detection of Ionizing Radiation

References

Chapter 3 Charge Collection and Signal Formation $\ldots$

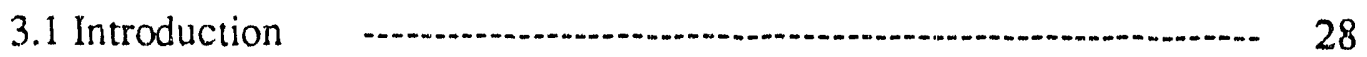

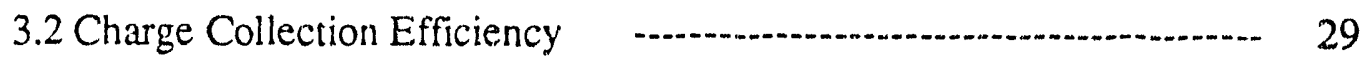

3.2.1 Case 1: Uniform Generation

3.2.2 Case 2 : P-side Surface Generation

3.2.3 Case $3: \mathrm{N}$-side Surface Generation

3.3 Signal Formation

3.3.1 Output Signal of Charge Sensitive Preamplifier

3.3.2 Output Signal of CR-(RC) ${ }^{\mathrm{n}}$ Shaping Amplifier

3.4 Input Parameters 
3.4.1 Average Ionization Energy

3.4.2 lonized Dangling Bond Density

3.4.3 Drift Mobility and Lifetime

3.5 Calculation Results and Measurements

3.5.1 Calculation Results

3.5.2 Signal Measurements

3.6 Discussion

References

Chapter 4 Electronic Noise in Detector-Amplifier System

4.1 Introduction 62

4.2 Noise of a-Si:H Detectors

4.2.1 Reverse Current in a-Si:H pin Diode

4.2.2 Noise Generation in a-Si:H pin Diode

4.3 Noise of Thin-Film-Transistor Amplifiers

4.3.1 a-Si:H and Poly-Si TFTs

4.3.2 Noise Sources in TFT's

4.4 Optimization of Noise by Pulse Shaping

4.4.1 Conversion of NPS to ENC

4.4.2 CR-(RC) ${ }^{\mathrm{n}}$ Pulse Shaping

4.4.3 Time-Variant Filter

4.5 Measurements and Calculation Results

4.5.1 Reverse Current and Noise of a-Si:H Detectors

4.5.2 I-V and Noise Power Spectrum of TFTs

4.6 Discussion

References

Chapter 5 Pixel Detector Readout Electronics

5.1 Introduction

5.2 a-Si:H Pixel (2-D) Detector Elecronics

5.2.1 Transistor-Switch Readout

5.2.2 Diode-Switch Readout

5.2.3 Pixel-Level Signal Amplification 
5.3 An a-Si:H Diode-Switch Readout

5.3.1 Design

5.3.2 Fabrication

5.3.3 Test and Results

5.4 A Poly-Si TFT Pixel Amplifier

123

5.4.1 Design

5.4.2 Fabrication

5.4.3 Test and Results

5.5 Discussion 136

References

Chapter 6 Conclusion

139

\section{Appendix}

A. A Program for Charge Collection and Signal Formation $\quad \ldots-\ldots 141$

B. Charge Collection Efficiency in a Multi-Buried-Layer Detector 146

C. A Summary of Thermal and Field-Enhanced Emission Rates - 151

D. Derivations of Noise Power Spectra

E. Evaluation of ENC for a CR-(RC) ${ }^{n}$ Filter

F. Time Domain Analysis of a Gated-Integrator Filter …-...-... 160 


\section{List of Figures}

\section{Chapter 2}

Fig. 2.1 Top views of a linear and a pixel array of a-Si:H radiation detectors.

Fig. 2.2 A schematic diagram of indium bump technique.

Fig. 2.3 A schematic diagram of signal pulse processing electronics for single detectors.

Fig. 2.4 Schematics of readout schemes from 1-dimensional detector array (a) delay line or resistive iinie readout scheme using two amplifiers (b) readout of signals from every detector strip or wire using the same number of amplifiers.

Fig. 2.5 Schematic diagrams of readout methods for a 2-dimensional pixel detector array (a) $x-y$ readout method (b) line scanning method .

Fig. 2.6 A schemaatic diagram of LBL PECVD system.

Fig. 2.7 A schematic structure of a-Si:H showing dangling bonds and a void.

Fig. 2.8 Schematic band structures of (a) crystalline and (b) amorphous silicon.

Fig. 2.9 Cross-sectional view of a single pixel ; (a) indirect detection scheme using a scintillating layer coupled to a thin a-Si:H photodiode and (b) direct detection scheme using a thick a-Si:H diode.

Fig. 2.10 Scintillation light spectra of pure $\mathrm{CsI}, \mathrm{CsI}(\mathrm{N} \varepsilon)$ and $\mathrm{CsI}(\mathrm{Tl})$ and detection efficiency of a-Si:H photodiodes.

\section{Chapter 3}

Fig. 3.1 A schematic geometry of an a-Si:H pin detector and the density distribution of generated charges; (a) surface generation at p-side and n-side, (b) uniform generation.

Fig. 3.2 A schematic diagram of space charge and electric field distribution in a partially depleted a-Si:H pin diode depicted by (a) two-region model and (b) one-region model.

Fig. 3.3 A schematic diagram of radiation detection system.

Fig. 3.4 A schematic diagram of experimental setup for time-of-flight measurement. Dye laser is pumped by $\mathrm{N}_{2}$ laser and produces light with the wave length $500 \sim 600$ $\mathrm{nm}$. 
Fig. 3.5 Electron time-of-flight data for a $27 \mu \mathrm{m}$ thick a-Si:H pin diode.

Fig. 3.6 Collected electron charges versus detector bias for a $27 \mu \mathrm{m}$ thick pin diode.

Fig. 3.7 Hole time-of-flight data for a $27 \mu \mathrm{m}$ thick a-Si:H pin diode.

Fig. 3.8 Collected hole charges versus detector bias for a $27 \mu \mathrm{m}$ thick a-Si:H pin diode.

Fig. 3.9 Electric field in i-layer of a-Si:H pin diode calculated for two different bias and densities of ionized dangling bonds.

Fig. 3.10 Calculated charge collection efficiency in detectors of the thickness up to $50 \mu \mathrm{m}$ at a fixed shaping time of $1 \mu \mathrm{sec}$.

Fig. 3.11 Calculated charge collection efficiency in a-Si:H pin detectors as a function of shaping time for three different biases. $\mathrm{n}=1$ was used.

Fig. 3.12 Calculated output signal pulse shapes of $C R-(R C)^{n}$ shaping amplifier with $n=1$ $\sim 4$ at $1 \mu \mathrm{sec}$ shaping time and a full depletion bias $(\mathrm{Y}=1)$.

Fig. 3.13 Calculated electric field of $50 \mu \mathrm{m}$ pin and buried pipin detectors under the reverse bias of $1400 \mathrm{~V}$ and $700 \mathrm{~V}$ respectively. Insertion is a schematic of pipin detector structure. Assumed ionized dangling bond density is $7 \times 10^{14} / \mathrm{cm}^{3}$.

Fig. 3.14 Calculated electron collection efficiency as a function of the number of the buried p-layers in a $50 \mu \mathrm{m}$ thick detector at a fixed bias of $675 \mathrm{~V}$. The number above the bar is the transit time.

Fig. 3.15 Collection efficiency vs bias of a $27 \mu \mathrm{m}$ thick sample for $760 \mathrm{~nm}$ light. Dots are measured data and lines are calculation results. Shaping time used is $9 \mu \mathrm{sec}$.

Fig. 3.16 Collection efficiency vs shaping time of a $27 \mu \mathrm{m}$ thick sample for $760 \mathrm{~nm}$ light. Dots are measured data and lines are calculation results. Applied bias is $600 \mathrm{~V}$.

Fig. 3.17 Collection efficiency of electrons and holes in the cases of surface generation for a $27 \mu \mathrm{m}$ thick sample. Dots are measured data by $510 \mathrm{~nm}$ laser light and lines are calculation results.

Fig. 3.18 Signal collection efficiency produced by $1 \mathrm{MeV}$ betas from $\mathrm{St}^{90}$ incident on a diode of thickness $27 \mu \mathrm{m}$. Shaping time used was $2.5 \mu \mathrm{sec}$.

\section{Chapter 4}

Fig. 4.1 Two sources of current in reverse-biased a-Si:H pin diodes. (1) Electron-hole pair generation through a mid-gap state and (2) electron tunneling injection from the $\mathrm{p}$-i interface through multistep hopping. 
Fig. 4.2 Mechanisms of electron generation from recombination centers (a) Simple thermal generation mechanism, (b) Three effects of strong electric field effects; (1) Poole-Frenkel barrier lowering, (2) phonon assisted tunneling and (3) direct tunneling.

Fig. 4.3 Calculated emission rates of Poole-Frenkel emission and tunneling from a Coulomb potential well with a $0.9 \mathrm{eV}$ barrier height at room temperature.

Fig. 4.4 A schematic diagram of the band structure near the conduction band mobility edge of the i-layer under reverse bias, showing the noise generation process due to trapping and detrapping of free electrons by shallow band-tail states.

Fig. 4.5 Schematic cross-sectional views of (a) a-Si:H and (b) poly-Si TFTs.

Fig. 4.6 Parallel and series noise sources in detector-amplifier system. (shot $=$ shot noise, $\mathrm{df}=$ detector $1 / \mathrm{f}$ noise, $\mathrm{tt}=$ transistor thermal noise and $\mathrm{tf}=$ transistor $1 / \mathrm{f}$ noise).

Fig. 4.7 Schematic representation of equivalent noise charges from various sources as a function of the shaping time; (a) detector shot noise $\sim \tau$, (b) detector $1 /$ f noise $\tau^{2}$, (c) series contact noise $\sim \tau^{-1}$, (d) amplifier thermal noise $\sim \tau^{-1}$, (e) amplifier $1 / \mathrm{f}$ noise, independent of $\tau$ and (f) total system noise.

Fig. 4.8 A schematic diagram of an gated-integrator and the time-variant pulse shaping.

Fig. 4.9 A schematic diagram of the correlated double sampling scheme.

Fig. 4.10 A schematic diagram of a-Si:H detector diode noise measurement setup of (A) noise power spectrum measurement and (B) equivalent noise charge measurement.

Fig. 4.11 Reverse current of a $26 \mu \mathrm{m}$ thick a-Si:H pin diode. To fit the measured data, we used $\beta_{P F}=0.066, \omega_{0}=10^{12}, E_{i}=0.9$ and $N_{t}=1.4 \times 10^{15}$.

Fig. 4.12 Activation energy of reverse biased a-Si:H pin diode $26 \mu \mathrm{m}$ thick.

Fig. 4.13 Measured output noise power spectrum of a $26 \mu \mathrm{m}$ thick a-Si:H pin diode. $1 / \mathrm{f}^{3}$ region corresponds to $1 / \mathrm{f}$ noise of at the input stage, and $1 / \mathrm{f}^{2}$ region is shot noise and the frequency independent noise is considered as a thermal noise generated at the contacts.

Fig. 4.14 (a) A schematic cross-sectional diagram of sample a-Si:H pin diode and (b) three noise sources in an equivalent circuit. Noise sources are A: 1/f noise, B: shot noise and $C$ : thermal noise from contact resistance. Large value of bulk resistance doesn't contribute significant noise.

Fig. 4.15 A plot of calculated noise current power coefficient $K_{0}$ for a $26 \mu \mathrm{m}$ thick pin dicde as a function of calculated Poole-Frenkel current. The calculation gives $\beta$ of 1.2 . 
Fig. 4.16 Measured $\mathrm{K}_{\mathrm{f}}$ for a-Si:H pin diodes of various detector thickness and calculated $\mathrm{K}_{\mathrm{f}}$ with the assumption of $\beta=1.2$.

Fig. 4.17 Reverse current and equivalent noise charge measurement of a reverse biased 26 $\mu \mathrm{m}$ thick a-Si:H pin diode and calculated ENC of (a) $1 / \mathrm{f}$ noise, (b) shot noise, (c) contaci noise, (d) amplifier noise and the total noise(solid line). Shaping time of $2.5 \mu \mathrm{sec}$ is used.

Fig. 4.18 Measure noise of a $26 \mu \mathrm{m}$ a-Si:H pin diode as a function of CR-RC shaping time. Detector $1 / \mathrm{f}$ noise is shown as a linearly increasing part of the total noise at high bias.

Fig. 4.19 A schematic diagram of TFT Noise measurement system. (Courtesy of Hung)

Fig. 4.20 Typical I-V curves of a-Si:H TFT made at Xerox. $W / L=128 / 16 \mu \mathrm{m}$.

Fig. 4.21 Measured 1/f noise spectrum of a-Si:H TFT made at Xerox. W/L $=128 / 16 \mu \mathrm{m}$ and the applied drain-to-source bias is $20 \mathrm{~V}$. Thermal noise is estimated from the measured values of transconductance $\mathrm{gm}$.

Fig. 4.22 Magnitude of $1 / \mathrm{f}$ noise power at $1 \mathrm{kHz}$ and drain-to-source current of a-Si:H TFT's as a function of channel dimension W/L.

Fig. $4.23 \mathrm{I}-\mathrm{V}$ curves of $900^{\circ} \mathrm{C}$ annealed $\mathrm{n}$-channel poly-Si TFT made at Xerox. W/L = $50 / 5 \mu \mathrm{m}$.

Fig. 4.24 Measured 1/f noise spectrum of $900^{\circ} \mathrm{C}$ annealed n-channel poly-Si TFT. W/L = $50 / 5 \mu \mathrm{m}$ and the applied drain-to-source bias is $10 \mathrm{~V}$. Thermal noise is estimated by the measured transconductance $\mathrm{gm}_{\mathrm{m}}$.

\section{Chapter 5}

Fig. 5.1 A-Si:H pin photodiode pixel array with a-Si:H TFT switching TFTs for readout data line by line. (Courtesy of Xerox PARC)

Fig. 5.2 A schematic diagram of 2-d pixel signal readout using a single diode.

Fig. 5.3 A Schematic diagram of an unit pixel detector with back-to-back readout diodes and a timing diagram showing the integration time $T_{i}$ and the readout time $T_{r}$.

Fig. 5.4 A schematic diagram of pixel detector and pixel level electronics consisting of an amplifier, signal hold and readout switch.

Fig. 5.5 A cross-sectional view of a two-diodes switching readout made at LBL.

Fig. 5.6 Measurement of forward and reverse current density of $2 \mu \mathrm{m}$ thick pin diode used for switching diodes. 
Fig. 5.7 A measurement setup for the signal charge transfer and loss from a pixel diode using a two-diodes switching readout method.

Fig. 5.8 Measurement of light signal as a function of delay time between the time of incidence of light and the time of measurement. (Measured RC time was 17 msec.)

Fig. 5.9 Measurement of light signal as a function of shaping time of CR-RC shaping amplifier. (RC time measured was $0.7 \mu \mathrm{sec}$.)

Fig. 5.10 The schematic circuit diagram of the prototype poly-Si CMOS charge-sensitive pixel amplifiers for a-Si:H pixel detectors. Each square with node numbers represents a test pad. $\mathrm{N}$ and $\mathrm{P}$ stand for $\mathrm{n}$-channel and p-channel TFTs, and $\mathrm{C}$ stands for capacitors.

Fig. 5.11 I-V Characteristics of n-channel poly-Si TFT. $W / L=50 / 5 \mu \mathrm{m}$.

Fig. 5.12 I-V Characteristics of $n$-channel poly-Si TFT. $W / L=30 / 5 \mu \mathrm{m}$.

Fig. 5.13 I-V Characteristics of $n$-channel poly-Si TFT. $W / L=50 / 10 \mu \mathrm{m}$.

Fig. 5.14 I-V Characteristics of p-channel poly-Si TFT. W/L=20/15 $\mu \mathrm{m}$.

Fig. 5.15 DC characteristics of the first stage with and without cascode configuration. $V_{d d}$ $=10 \mathrm{~V}, \mathrm{~V}_{\mathrm{p}}=5 \mathrm{~V}$ and $\mathrm{V}_{\mathrm{c}}=5 \mathrm{~V}$.

Fig. 5.16 Gain and frequency curves of the first stage with and without cascode configuration. $\mathrm{V}_{\mathrm{dd}}=10 \mathrm{~V}, \mathrm{~V}_{\mathrm{p}}=5 \mathrm{~V}$ and $\mathrm{V}_{\mathrm{c}}=5 \mathrm{~V}$.

Fig. 5.17 Measured noise power spectrum of the front-end n-channel poly-Si TFT. W/L $=50 / 5 \mu \mathrm{m}$ at $\mathrm{V}_{\mathrm{gs}}=1.8 \mathrm{~V}, \mathrm{~V}_{\mathrm{ds}}=4 \mathrm{~V}$. (operation bias level). $\mathrm{gm}_{\mathrm{m}}=\sim 20 \mu \mathrm{A} / \mathrm{V}$.

Fig. 5.18 Calculated equivalent noise charge of the front-end n-channel poly-Si TFT as a function of the shaping time for $\mathrm{CR}-(\mathrm{RC})^{\mathrm{n}}$ shaping amplifier with $\mathrm{n}=1$ and $\mathrm{n}=$ 4. $W / L=50 / 5 \mu \mathrm{m}$ and $V_{g s}=1.8 \mathrm{~V}, V_{d s}=4 \mathrm{~V}$. (operation bias level). $\mathrm{g}_{\mathrm{m}}=\sim$ $20 \mu \mathrm{A} / \mathrm{N}$.

Fig. 5.19 DC characteristics of 2 nd stage with and without the cascode configuration.

Fig. 5.20 Total voltage gain of the complete amplifier with and without the cascode configuration. 


\section{List of Tables}

Table 2.1 Deposition parameters of LBL PECVD system

Table 2.2 Material properties of crystalline and hydrogenated amorphous silicon

Table 3.1 Material parameters of a-Si:H pin diodes

Table 3.2 Relative collection efficiency in a-Si:H as compared with crystal $\mathrm{Si}$

Table 4.1 The coefficient $\alpha$ and $\beta$ for various amorphous silicon devices

Table 4.2 Equivalent noise charges (electrons in $\mathrm{mm}$ ) from detector-amplifier system

Table 4.3 A summary of basic features of a-Si:H and poly-Si TFTs

Table 5.1 Integration process for a two-diodes switch coupled to pixel diodes

Table 5.2 Input parameters of n-channel and p-channel poly-Si TFT for PSPICE

Table 5.3 A list of components and their dimensions

Table $5.4 \quad$ Nodes

Table 5.5 Measured circuit parameters of poly-Si TFTs 


\section{List of Symbols}

\section{Chapter 3}

Symbol : Meaning ordefinition Unit

Ac : Closed-loop gain of charge sensitive amplifier

Ao : Open-loop gain of charge sensitive amplifier

a : Absorption coefficient of the incident radiation or light

$[/ \mathrm{cm}]$

$\alpha \quad:$ Dispersion Coefficient

$\alpha_{e} \quad:$ Parameter defined as $\left(\tau_{n}+\tau_{c}\right) /\left(\tau_{n} \tau_{e}\right)$

[1/sec]

$\alpha_{h} \quad:$ Parameter defined as $\left(\tau_{p}+\tau_{h}\right) /\left(\tau_{p} \tau_{h}\right)$

$[1 / \mathrm{sec}]$

$\beta_{e} \quad:$ Parameter defined as $\left(\tau_{n}-\tau_{e}\right) /\left(\tau_{n} \tau_{e}\right)$

$[1 / \mathrm{sec}]$

$\beta_{h} \quad:$ Parameter defined as $\left(\tau_{p}-\tau_{h}\right) /\left(\tau_{p} \tau_{h}\right)$

$[1 / \mathrm{sec}]$

C : Capacitance of CR-RC shaping amplifier

[Farad]

$\mathrm{C}_{\mathrm{d}}:$ Detector capacitance [Farad]

$\mathrm{C}_{\mathrm{f}}:$ Feedback crpacitance of charge sensitive preamplifier [Farad]

$\mathrm{C}_{\mathrm{i}} \quad$ : Input capacitance of charge sensitive preamplifier [Farad]

$\mathrm{C}_{\mathrm{tot}}$ : Sum of detector capacitance and input capacitance of preamplifier [Farad]

d : Thickuess of the i-layer in pin detectors

$[\mu \mathrm{m}]$

$\delta(\mathrm{x}) \quad$ : Delta function

$\mathrm{E}_{\mathrm{g}} \quad: \quad$ Bandgap energy of semiconductor

$\Delta \mathrm{E}(\mathrm{x}) \quad$ : Energy deposited at position $\mathrm{x}$ by an incident raćsation $=\mathrm{dE} / \mathrm{dx}$

$\varepsilon_{\text {asi }} \quad: \quad$ Relative dielectric constant of amorphous silicon $=11.8$

$\varepsilon_{0} \quad:$ Dielectric constant of vacuum $=8.854 \times 10^{-14}$

$[\mathrm{F} / \mathrm{cm}]$

$\mathrm{F}(\mathrm{x}) \quad$ : Electric field strength at position $\mathrm{x}$ in i layer of pin diode $\quad[\mathrm{V} / \mathrm{cm}]$

$\mathrm{F} \quad:$ Uniform electric field in the time-of-flight measurement $\quad[\mathrm{V} / \mathrm{cm}]$

$\Phi(\mathrm{x})$ : Electric field distribution in the i-layer [V]

$\Phi_{\mathrm{c}} \quad$ : Critical potential where the deep depletion ends in the i-layer

$[\mathrm{V}]$

$\eta \quad$ : Total collection efficiency

$\eta_{\mathrm{e}} \quad$ : Electron collection efficiency

$\eta_{\mathrm{h}} \quad$ : Hole collection efficiency

$\mathrm{I}_{\mathrm{e}} \quad$ : Induced photocurrent due to electron drift motion

Iind : Induced current from a-Si:H pin detector [Amp]

i : Induced current due to motion of a single charge 
$\Delta \mathrm{I}_{\mathrm{e}} \quad$ : Induced current due to the drift of electrons in the i-layer

[Amp/cm]

$\Delta \mathrm{I}_{\mathrm{h}} \quad$ : Induced current due to the drift of signal holes in the i-layer

[Amp/cm]

the : Mobility of electrons in i layer of a-Si:H

$\left[\mathrm{cm}^{2} / \mathrm{Vsec}\right]$

$\mu_{\mathrm{h}} \quad$ : Mobility of holes in i layer of a- $\mathrm{Si}: \mathrm{H}$

$\left[\mathrm{cm}^{2} / \mathrm{Vsec}\right]$

$\mathrm{N} \quad$ : Number of moving charge

[\#]

No : Total number of generated electron-hole pairs

$\mathrm{N}_{\mathrm{d}}{ }^{*} \quad$ : Ionized dangling bond density of the i-layer

$\left[\mathrm{cm}^{-3}\right]$

$\mathrm{n} \quad:$ Number of differential stage in $\mathrm{CR}-(\mathrm{RC})^{\mathrm{n}}$ shaping amplifier

$n_{0} \quad$ : Density of uniformly generated electron-hole pairs [ $\left.\mathrm{cm}^{-1}\right]$

$\mathrm{n}_{\mathrm{O}}(\mathrm{x})$ : Density of generated electron-hole pairs per unit thickness at $\mathrm{x}$ [ $\left.\mathrm{cm}^{-1}\right]$

Qcoi : Total collected charge

[Coul]

Qgen : Total generated charge by an incident radiation

[Coul]

Qe : Induced charge due to the collection of signal electrons

[Coul]

Qh : Induced charge due to the collection of signal holes

[Coul]

Qo : Total generated charges $=\mathrm{q} \mathrm{N}_{\mathrm{o}}$

[Coul]

$\Delta Q_{e}\left(x_{0}\right)$ : Induced charge due to the collection of electrons generated at $x_{0}$

[Coul]

$\Delta Q_{h}\left(x_{0}\right):$ Induced charge due to the collection of holes generated at $x_{0}$

[Coul]

$\mathrm{q} \quad$ : Electronic charge $=1.602 \times 10^{-19}$

[Coul]

R : Resistance of CR-RC shaping amplifier

[Ohm]

$R_{f} \quad:$ Feedback resistance of charge sensitive preamplifier

[Ohm]

$\rho$

: Slope the electric field in the deep depletion region of the i-layer

$\left[\mathrm{V} / \mathrm{cm}^{2}\right]$

T : Temperature in Kelvin

$T_{i} \quad$ : Integration time of the induced current $=$ the lesser between $\tau$ and $t_{t}$

To : Width of valence band tail states

$\mathrm{t} \quad$ : Time variable

[sec]

$\mathrm{t}_{\mathrm{n}} \quad$ : Electron transit time from $\mathrm{x}=0$ to $\mathrm{x}=\mathrm{d}$

$t_{p}$ : Hole transit time from $x=x_{0}$ to $x=0$

$t_{t} \quad:$ Transit time of charge carriers under the uniform field

$\tau$ : Shaping time of CR-RC pulse shaping amplifier

$\tau_{f} \quad:$ Pulse decay time of charge sensitive preamplifier $=R_{f} \times C_{f}$

$\tau_{e} \quad:$ Electron Lifetime in the i-layer of a-Si:H

$\tau_{n} \quad:$ Electron characteristic time $=1 / p \mu_{e}$

$\tau_{h} \quad:$ Hole Lifetime in the i-layer of a-Si:H

$\tau_{p} \quad$ : Hole characteristic time $=1 / \rho \mu_{\mathrm{h}}$

$\mathrm{V}_{\mathrm{a}} \quad$ : Reverse bias of pin diode

$V_{f} \quad:$ Full depletion bias of the i-layer $=7 d^{2} / 2$ 


$\begin{array}{lllr}\mathrm{V}_{\mathrm{i}} & : \text { Input signal of the preamplifier without a feedback loop } & \text { [Volt] } \\ \mathrm{V}_{\mathrm{csa}} & : \text { Output signal of charge sensitive preamplifier } & \text { [Volt] } \\ \mathrm{V}_{\text {sha }} & : \text { Output pulse of shaping amplifier } & \text { [Volt] } \\ \mathrm{v}(\mathrm{x}) & : \text { Drift velocity of charge carriers at position } \mathrm{x} & {[\mathrm{cm} / \mathrm{sec}]} \\ \mathrm{v}_{\mathrm{e}} & : \text { Drift velocity of electron in the i-layer } & {[\mathrm{cm} / \mathrm{sec}]} \\ \mathrm{v}_{\mathrm{h}} & : \text { Drift velocity of hole in the i-layer } & {[\mathrm{cm} / \mathrm{sec}]} \\ \mathrm{W} & : \text { Average ionization energy to produce an electron-hole pair } & {[\mathrm{eV}]} \\ \mathrm{w} & : \text { Depletion thickness of the i-layer in pin diode }=\left(2 \mathrm{~V}_{\mathrm{a}} / \mathrm{p}\right)^{1 / 2} & {[\mu \mathrm{m}]} \\ \mathrm{x} & : \text { Spatial variable of the i-layer measured from the p-i contact } & {[\mu \mathrm{m}]} \\ \mathrm{x}_{\mathrm{O}} & : \text { Position of charge generation in the i-layer from the p-i contact } & {[\mu \mathrm{m}]} \\ \mathrm{Y} & : \text { Depletion parameter }=\mathrm{w} / \mathrm{d}=\left(\mathrm{V}_{\mathrm{a}} / \mathrm{V}_{\mathrm{f}}\right)^{1 / 2} & \end{array}$

\section{Chapter 4}

Symbol : Meaning or definition Unit

A : Cross-sectional area of the pin detector diode $\quad\left[\mathrm{cm}^{2}\right]$

As : Normalization constant of conversion process from NPS to ENC

A sys : Charge-to-voltage conversion gain of the measurement system $\left[\mathrm{V}^{2} / \mathrm{Coul}^{2}\right]$

$A_{w b} \quad:$ Gain of wide-band amplifier used in the measurement

$\mathrm{A}_{\alpha} \quad: \quad$ Normalization constant of $1 / \mathrm{f}^{\alpha}$ noise

a : Inverse of $\mathrm{RC}$ constant of $\mathrm{CR}-(\mathrm{RC})^{\mathrm{n}}$ shaping amplifier

$\left[\sec ^{-1}\right]$

$\alpha \quad: \quad$ Frequency dependency factor of flicker noise of a-Si:H pin diode

$\alpha_{H} \quad:$ Hooge's parameter

$\beta \quad$ : Current dependency factor of flicker noise

$\beta_{\mathrm{d}} \quad:$ Current dependency factor of flicker noise of a-Si:H pin diodes $\quad\left[\mathrm{eV} / \mathrm{F}^{0.5}\right]$

$\beta_{l} \quad:$ Current dependency factor of flicker noise of TFTs $\quad$ [eV/F0.5]

$\beta_{\mathrm{PF}}:$ Poole-Frenkel emission coefficient $\quad[\mathrm{eV} / \mathrm{F} 0.5]$

$\mathrm{C}_{c} \quad:$ Coupling capacitance between a preamp and sample-\&-hold circuit [Farad]

$\mathrm{C}_{\mathrm{d}}:$ Equivalent capacitance of a-Si:H detector [Farad]

$\mathrm{C}_{\mathrm{i}} \quad$ : Input capacitance of the front-end TFT in a preamplifier [Farad]

$\mathrm{C}_{\text {in }}:$ Dynamic input capacitance of the charge sensitive amplifier [Farad]

$\mathrm{C}_{\mathrm{h}} \quad:$ Capacitance signal storing capacitor in sample-\&-hold circuit [Farad]

$\mathrm{C}_{\mathrm{O}}:$ Capacitance of unit area of the gate insulator $\quad\left[\mathrm{F} / \mathrm{cm}^{2}\right]$

$\mathrm{C}_{\text {tot }}:$ Total input capacitance of a detector and amplifier combination [Farad]

d : Thickness of the i-layer in pin detectors $\quad[\mu \mathrm{m}]$

e : Base of natural logarithrns $=2.73$ 


\begin{tabular}{|c|c|c|c|}
\hline$e_{0}$ & $:$ & Thermal emission rate of electrons from deep states & {$\left[\mathrm{sec}^{-1}\right]$} \\
\hline$e_{P F}$ & : & Poole-Frenkel emission rate of electrons from deep states & {$\left[\sec ^{-1}\right]$} \\
\hline ETH & $:$ & Thermal emission rate of electrons for a barrier height $\mathrm{E}_{\mathrm{i}}$ & {$\left[\sec ^{-1}\right]$} \\
\hline$(\mathrm{ENC})_{j}$ & : & Equivalent noise charge due to noise component $\mathrm{j}$ & [electrons] \\
\hline $\mathrm{E}_{\mathrm{a}}$ & : & Activation energy of reverse current of a-Si:H pin diodes & [eV] \\
\hline$E_{c}$ & : & Conduction band mobility edge & {$[\mathrm{eV}]$} \\
\hline $\mathrm{E}_{\mathrm{f}}$ & : & Fermi energy level of electrons, traps or recombination centers & {$[\mathrm{eV}]$} \\
\hline $\mathrm{E}_{\mathrm{i}}$ & : & Thermal emission barrier $=E_{c}-E_{t}$ & {$[\mathrm{eV}]$} \\
\hline $\mathrm{E}_{\mathrm{l}}$ & : & Energy level of deep traps or recombination centers & {$[\mathrm{eV}]$} \\
\hline $\mathrm{E}_{\mathrm{v}}$ & : & Valence band mobility edge & {$[\mathrm{eV}]$} \\
\hline$\Delta \mathrm{E}$ & : & Coulomb Barrier lowering due to the external field & {$[\mathrm{eV}]$} \\
\hline$\varepsilon_{\mathrm{i}}$ & : & Relative dielectric constant of the gate insulator of TFTs & \\
\hline$\varepsilon_{0}$ & : & Dielectric constant of vacuum $=8.854 \times 10^{-14}$ & {$[\mathrm{~F} / \mathrm{cm}]$} \\
\hline $\mathrm{F}$ & : & Fano factor $\approx 0.1$ for $\mathrm{Si}$ and $\approx 0.06$ for $\mathrm{Ge}$ & \\
\hline$F(x)$ & : & Electric field at position $\mathrm{x}$ & {$[\mathrm{V} / \mathrm{cm}]$} \\
\hline $\mathrm{F}_{\mathrm{m}}$ & : & Minimum electric field in a pin detector diode & {$[\mathrm{V} / \mathrm{cm}]$} \\
\hline$F_{0}$ & : & Peak electric field at the $\mathrm{p}-\mathrm{i}$ junction in a pin detector diode & {$[\mathrm{V} / \mathrm{cm}]$} \\
\hline f & : & Frequency & {$[\mathrm{Hz}]$} \\
\hline$f(E)$ & : & Fermi distribution function of a trap at energy $E$ & \\
\hline$f_{\min }$ & : & Minimum frequency of $1 / f$ noise region & {$[\mathrm{Hz}]$} \\
\hline$f_{\max }$ & : & Maximum frequency of $1 / \mathrm{f}$ noise region & {$[\mathrm{Hz}]$} \\
\hline$f_{0}$ & : & Characteristic frequency of generation-recombination noise & {$[\mathrm{Hz}]$} \\
\hline$f_{t}$ & * & Fermi distribution function of traps or recombination centers & \\
\hline$\Delta \mathrm{f}$ & : & Frequency band & [Hz] \\
\hline$G(f)$ & : & Transfer function of shaping amplifier & \\
\hline$G_{n}(f)$ & : & Transfer function of $C R-(R C)^{n}$ shaping amplifier & \\
\hline$g_{m}$ & : & Transconductance of TFTs & [Amp] \\
\hline$\overline{\mathrm{h}}$ & : & Reduced Planck constant $=h / 2 \pi=1.05459 \times 10^{-27}$ & {$[\mathrm{erg} / \mathrm{sec}]$} \\
\hline 1 & : & Current in an ohmic device & [Amp] \\
\hline $\mathrm{I}(\mathrm{V})$ & : & Current of diode at bias V & [Amp] \\
\hline $\mathrm{I}_{\mathrm{O}}$ & : & Reverse saturation current of diodes & [Amp] \\
\hline $\mathrm{I}_{\mathrm{R}}$ & : & Reverse current of pin diode & [Amp] \\
\hline $\mathrm{I}_{\mathrm{BG}}$ & : & Bulk generated current & [Amp] \\
\hline $\mathrm{I}_{\mathrm{nH}}$ & : & Thermal generation current in the bulk i-layer & [Amp] \\
\hline$I_{e}(x)$ & : & Electron current component at position $x$ & [Amp] \\
\hline$I_{h}(x)$ & & Hole current component at position $\mathrm{x}$ & [Amp] \\
\hline
\end{tabular}


$I_{d s} \quad:$ Drain-to-source current of TFTs

[Amp]

$\left\langle i^{2}\right\rangle \quad$ : Parallel current noise source of detector system

[Amp $\left.{ }^{2}\right]$

$\left\langle i^{2}{ }_{\text {in }}\right\rangle$ : Input noise current power spectrum of a detector

$\left[\mathrm{Amp}^{2}\right]$

$<i^{2}$ shot $>$

: Shot noise component of detector

[Amp ${ }^{2}$ ]

$<\mathrm{i}^{2} 1 / \mathrm{f}>$ : Flicker noise component of detector

[Amp ${ }^{2}$ ]

$\Delta \mathrm{I} \quad$ : Fluctuation in the current

[Amp]

$\mathrm{K}_{\mathrm{cv}} \quad$ : Charge-to-voltage conversion gain of the measurement

[V/Coul]

$\mathrm{K}_{\mathrm{f}} \quad$ : Coefficient of $1 / \mathrm{f}$ noise of a-Si:H pin diodes

$\mathrm{K}_{0} \quad: \quad$ Coefficient of $1 / \mathrm{f}$ noise of a-Si:H pin diodes including current term

$\mathrm{K}_{\mathrm{S}} \quad$ : Measured coefficient of shot noise of a-Si:H pin diodes

[Coul]

$\mathrm{K}_{\mathrm{t}} \quad$ : Coefficient of $1 / \mathrm{f}$ noise of a-Si:H TFTs

k : Boltzmann constant $=0.8617 \times 10^{-4}$

$[2 \mathrm{~V} / \mathrm{K}]$

L. : Channel length of TFTs

$m_{*} \quad$ : Effective mass of electrons at a trap

$\mu \quad$ : Mobility of charge carriers

$\left[\mathrm{cm}^{2} / \mathrm{Vsec}\right]$

$\mu_{c} \quad: \quad$ Electron extended mobility

$\mu_{\mathrm{e}} \quad$ : Electron drift mobility

$\left[\mathrm{cm}^{2} / \mathrm{Vsec}\right]$

$\mu_{\mathrm{fe}} \quad:$ Field effect mobility of TFTs

$\left[\mathrm{cm}^{2} / \mathrm{Vsec}\right]$

$\mu_{\mathrm{h}} \quad$ : Hole drift mobility

$\left[\mathrm{cm}^{2} / \mathrm{Vsec}\right]$

$\mu_{\mathrm{v}} \quad$ : Hole extended mobility

$\left[\mathrm{cm}^{2} / \mathrm{Vsec}\right]$

$\Delta \mu \quad: \quad$ Fluctuation in the mobility of charge carriers

$\mathrm{N}$ : Total number of charge carriers in an ohmic device

$\left[\mathrm{cm}^{2} / \mathrm{Vsec}\right]$

$\mathrm{N}_{\mathrm{r}} \quad$ : Number of electrons at recombination centers

[\#]

$\mathrm{N}_{\mathrm{c}} \quad$ : Density of states in the conduction band

$\mathrm{N}_{\mathrm{O}} \quad$ : Density of shallow trap states at energy $\mathrm{E}_{\mathrm{o}}$ below $\mathrm{E}_{\mathrm{c}}$

$\left[\mathrm{cm}^{-3} \mathrm{eV}^{-1}\right]$

$\mathrm{N}_{\mathrm{t}}$

Density of trap states near Fermi energy level

$\left[\mathrm{cm}^{-3} \mathrm{eV}^{-1}\right]$

$N_{1}(E) \quad$ : Density of shallow trap states $E_{o}<E<E_{c}$

$\left[\mathrm{cm}^{-3} \mathrm{eV}^{-1}\right]$

$\mathrm{N}_{2}(\mathrm{E}) \quad$ : Density of shallow trap states $\mathrm{E}<\mathrm{E}_{\mathrm{O}}$

$\left[\mathrm{cm}^{-3} \mathrm{eV}^{-1}\right]$

$N(E) \quad$ : Distribution function of recombination centers in the i-layer

$\left[\mathrm{cm}^{-3} \mathrm{eV}^{-1}\right]$

$\mathrm{N}_{\mathrm{j}} \quad$ : Equivalent noise charge of component $\mathrm{j}$

$\left[\mathrm{cm}^{-3} \mathrm{eV}^{-1}\right]$

$\mathrm{N}_{1 / \mathrm{f}} \quad$ : Equivalent noise charge of $1 / \mathrm{f}$ noise component of detector

[electrons]

$N_{\text {amp }} \quad$ : Electronic noise of the amplifier and pulse processing system

[electrons]

$N_{\text {det }}:$ Electronic noise of a detector

[electrons]

[electrons]

$\mathrm{N}_{\text {shot }}$ : Equivalent noise charge of shot noise component of a detector

[electrons]

$\mathrm{N}_{\mathrm{Sr}} \quad$ : Equivalent noise charge due to series resistance of a detector

[electrons]

$\mathrm{N}_{\text {sta }}$

Statistical fluctuation of radiation signal

[electrons] 


\begin{tabular}{|c|c|c|c|}
\hline $\mathrm{N}_{\text {sys }}$ & & Detector-Amplifier system noise & [electrons] \\
\hline $\mathrm{N}_{\text {tot }}$ & & Total noise of a detection system & [electrons] \\
\hline$(\mathrm{NPS})_{\mathrm{j}}$ & & Noise power spectrum of noise component $j$ & {$\left[\right.$ Volt $\left.^{2}\right]$} \\
\hline $\mathrm{n}(\mathrm{E})$ & & Density of trapped electrons at energy $E$ & {$\left[/ \mathrm{cm}^{3}\right]$} \\
\hline $\mathrm{n}_{1}$ & & Density of shallow trapped electrons $E_{0}<E<E_{c}$ & {$\left[/ \mathrm{cm}^{3}\right]$} \\
\hline $\mathrm{n}_{2}$ & & Density of shallow trapped electrons $E<E_{0}$ & {$\left[/ \mathrm{cm}^{3}\right]$} \\
\hline $\mathrm{n}_{\mathrm{f}}$ & & Density of free electrons in the i-layer of pin diode & {$\left[/ \mathrm{cm}^{3}\right]$} \\
\hline $\mathrm{n}_{\mathbf{t}}$ & & Density of shallow trapped electrons in the i-layer of pin diode & {$\left[/ \mathrm{cm}^{3}\right]$} \\
\hline$n_{\text {tot }}$ & & Density of total electrons in the conduction band of the i-layer & {$\left[/ \mathrm{cm}^{3}\right]$} \\
\hline$\Delta \mathrm{N}$ & & Fluctuation in the number of charge carriers & [\#] \\
\hline$\Delta \mathrm{N}_{\mathrm{r}}$ & & Fluctuation in the number of electrons in recombination centers & [\#] \\
\hline$\overline{\Delta n(E)^{2}}$ & : & Variation of $n(E)$ & {$\left[/ \mathrm{cm}^{3}\right]$} \\
\hline$q$ & & Electronic charge $=1.602 \times 10^{-19}$ & [Coul] \\
\hline Qcol & : & Total collected charge & [Coul] \\
\hline$\theta$ & : & Coefficient of thermal noise of TFTs & \\
\hline$\theta_{1}$ & & Parameter defined as $\theta_{1}=T_{1} /\left(T_{1}-T\right)$ & \\
\hline$\theta_{2}$ & & Parameter defined as $\theta_{2}=\mathrm{T}_{2} /\left(\mathrm{T}-\mathrm{T}_{2}\right)$ & \\
\hline $\mathrm{R}_{\mathrm{e}}$ & & Ratio of shallow trapped electron density to free electron density = & $=\mathrm{n}_{\mathrm{V}} / \mathrm{n}_{\mathrm{f}}$ \\
\hline $\mathrm{R}_{\mathrm{h}}$ & : & Ratio of shallow trapped hole density to free hole density $=\mathrm{p} / \mathrm{pf}$ & \\
\hline $\mathrm{R}_{\mathrm{S}}$ & & Series contact resistance of a-Si:H pin diode & [Ohm] \\
\hline$\rho$ & & Slope the electric field in the deep depletion region of the i-layer & {$\left[\mathrm{V} / \mathrm{cm}^{2}\right]$} \\
\hline$S_{1 / \mathrm{r}}(\mathrm{f})$ & & 1/f noise spectral power of a-Si:H pin diode $=\left\langle\mathrm{i}^{2} 1 / \mathrm{f}\right\rangle / \Delta \mathrm{f}$ & {$\left[\mathrm{Amp}^{2} / \mathrm{Hz}\right]$} \\
\hline$S_{\text {det }}(f)$ & & Detector noise current spectral power & {$\left[\mathrm{Amp}^{2} / \mathrm{Hz}\right]$} \\
\hline$S_{g r}(f)$ & & Generation-recombination noise spectral power & {$\left[\mathrm{Amp}^{2} / \mathrm{Hz}\right]$} \\
\hline$S_{l}(f)$ & & Spectral power of the noise current & {$\left[\mathrm{Amp}^{2} / \mathrm{Hz}\right]$} \\
\hline$S_{I-e}(f)$ & & Spectral power of the noise current due to electron fluctuation & {$\left[\mathrm{Amp}^{2} / \mathrm{Hz}\right]$} \\
\hline$S_{I-h}(f)$ & & Spectral power of the noise current due to hole fluctuation & {$\left[\mathrm{Amp}{ }^{2} / \mathrm{Hz}\right]$} \\
\hline$S_{\mu}(f)$ & & Spectral power of the fluctuation in the mobility $\left[\mathrm{cm}^{4} /\right.$ & $\left.4 / V^{2} \sec ^{2} H z\right]$ \\
\hline$S_{N}(f)$ & : & Spectral power of the fluctuation in the number of charge carriers & {$[\# / H z]$} \\
\hline$S_{n f}(f)$ & & Spectral power of the fluctuation in $n_{f}$ & {$[\# / \mathrm{Hz}]$} \\
\hline$S_{n_{l}}(f)$ & & Spectral power of the fluctuation in $n_{t}$ & {$[\# / \mathrm{Hz}]$} \\
\hline$S_{\text {sig }}$ & & Detector signal charge or collected charge & [electrons] \\
\hline$S_{\text {shot }}(f)$ & & Shot noise spectral power $=\left\langle\mathrm{i}^{2}\right.$ shot $\rangle / \Delta \mathrm{f}$ & {$\left[\mathrm{Amp} \mathrm{P}^{2} / \mathrm{Hz}\right]$} \\
\hline$S_{s r}(f)$ & & Thermal noise spectral power of series resistance & {$\left[\mathrm{Volt}^{2} / \mathrm{Hz}\right]$} \\
\hline $\mathrm{S}_{\text {sys }}(\mathrm{f})$ & & Detector-amplifier system noise spectral power & {$\left[\mathrm{Volt}^{2} / \mathrm{Hz}\right]$} \\
\hline
\end{tabular}




\begin{tabular}{|c|c|c|c|}
\hline$s_{\mathrm{ru}}(f)$ & & $1 / \mathrm{f}$ noise spectral power of TFTs & {$[$ Volt $2 / \mathrm{Hz}]$} \\
\hline$S_{\text {TFT }}(f)$ & & TFT noise spectral power $=\left\langle\mathrm{v}^{2} \mathrm{TFT}>/ \Delta \mathrm{f}\right.$ & {$\left[\mathrm{Voltt}^{2} / \mathrm{Hz}\right]$} \\
\hline$S_{\text {th }}(f)$ & & Thermal noise spectral power of TFTs & {$\left[\right.$ Volt $\left.^{2} / \mathrm{Hz}\right]$} \\
\hline$S_{v}(f)$ & & Spectral power of the noise voltage & {$[\mathrm{Amp} 2 / \mathrm{Hz}]$} \\
\hline$\Delta S_{\mathrm{i}-\mathrm{e}}(\mathrm{x})$ & & Fluctuation of electron current component at position $\mathrm{x}$ & [Amp] \\
\hline$\Delta \mathrm{S}_{\mathrm{i}-\mathrm{h}}(\mathrm{x})$ & & Fluctuation of hole current component at position $\mathrm{x}$ & [Amp] \\
\hline$\Delta S_{n(E)}$ & & Spectral power of fluctuation in $n(E)$ & {$[\# / \mathrm{Hz}]$} \\
\hline $\mathbf{s}$ & & Average distance between two neighboring recombination centers & [cm] \\
\hline $\mathrm{T}$ & & Temperature & {$\left[{ }^{\circ} \mathrm{K}\right]$} \\
\hline $\mathrm{T}_{1}$ & & Characteristic constant to describe the slope of shallow states $N_{1}$ & {$\left[{ }^{\circ} \mathrm{K}\right]$} \\
\hline $\mathrm{T}_{2}$ & & Characteristic constant to describe the slope of shallow states $\mathrm{N}_{2}$ & {$\left[{ }^{\circ} \mathrm{K}\right]$} \\
\hline $\mathrm{T}_{\mathrm{i}}$ & & Integration time of a gated integrator & [sec] \\
\hline $\mathrm{T}_{\mathrm{i} 1}$ & & First integration time of correlated double sampling & [sec] \\
\hline $\mathrm{T}_{\mathrm{i} 2}$ & & Second integration time of correlated double sampling & [sec] \\
\hline$\Delta \mathrm{T}$ & 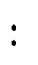 & Time interval between two sampling in correlated double sampling & [sec] \\
\hline $\mathrm{t}$ & $:$ & Time variable & [sec] \\
\hline$t_{i}$ & : & Thickness of the gate insulator & [cm] \\
\hline$\tau$ & $:$ & Shaping time of pulse shaping amplifier & [sec] \\
\hline$\tau$ & : & Characteristic emission time constant $=1 / \mathrm{e}_{0}=\exp \left[\left(\mathrm{E}_{\mathrm{c}}-\mathrm{E}\right) / \mathrm{kT}\right] / \omega_{0}$ & [sec] \\
\hline$\tau_{\mathrm{c}}$ & : & $=1 / \omega_{0}$ & [sec] \\
\hline$\tau_{\mathrm{f}}$ & : & $=\exp \left[\left(E_{c}-E_{f}\right) / k T\right] / \omega_{0}$ & [sec] \\
\hline$\tau_{0}$ & $:$ & $=\exp \left[\left(E_{c}-E_{0}\right) / k T\right] / \omega_{0}$ & [sec] \\
\hline $\mathrm{U}$ & : & Characteristic energy for tunneling from a trap to conduction band & {$[\mathrm{eV}]$} \\
\hline $\mathrm{V}_{\mathrm{a}}$ & : & Applied reverse bias of pin diode & [Volt] \\
\hline$V_{\text {clamp }}$ & : & Reference voltage of correlated double sampling circuit & [Volt] \\
\hline $\mathrm{V}_{\mathrm{gs}}$ & : & Gate-to-source bias of 'TFTs & [Volt] \\
\hline $\mathrm{V}_{\mathrm{T}}$ & : & Threshold voltage of TFTs & [Volt] \\
\hline$\left\langle v^{2}\right\rangle$ & : & Series voltage noise source of detector system & [Volt $\left.{ }^{2}\right]$ \\
\hline$\left\langle v^{2}\right.$ out $\rangle$ & : & Measured output noise power spectrum & [Volt $\left.{ }^{2}\right]$ \\
\hline$<v^{2}$ sr $>$ & : & Series resistance noise component of a detector & [Volt $\left.{ }^{2}\right]$ \\
\hline$\left\langle v^{2}{ }_{\mathrm{tP}}\right\rangle$ & : & Flicker noise component of a TFT & {$\left[\right.$ Volt $\left.{ }^{2}\right]$} \\
\hline$\left\langle v^{2} t h\right\rangle$ & : & Thermal noise component of a TFT & [Volt $\left.{ }^{2}\right]$ \\
\hline W & : & Channel width of TFTs & [cm] \\
\hline$\omega$ & : & Angular frequency $=2 \pi f$ & [rad] \\
\hline $\min$ & & Minimum angular frequency of $1 / \mathrm{f}$ region in a-Si: $\mathrm{H}$ diodes & [rad] \\
\hline & & Emission prefactor or a trial-and-escape probability & [sec \\
\hline
\end{tabular}


$\mathbf{x} \quad$ : Spatial variable of the i-layer measured from the $\mathrm{p}-\mathrm{i}$ contact

$[\mu \mathrm{m}]$

$\mathrm{Z}_{\mathrm{e}} \quad$ : Ratio of shallow trapped electron density $\mathrm{n}_{2}$ to free electron density $=\mathrm{n}_{2} / \mathrm{n}_{\mathrm{f}}$

$Z_{h} \quad$ : Ratio of shallow trapped holes density $p_{2}$ to free hole density $=p_{2} / p_{f}$

z : Parameter defined as $\mathrm{z}=\omega \tau$

$\mathrm{z}_{\mathrm{c}} \quad: \quad=\omega \tau_{\mathrm{c}}$

$\mathrm{z}_{\mathrm{f}} \quad:=\omega \tau_{\mathrm{f}}$

$z_{0} \quad:=\omega \tau_{0}$

\section{Chapter 5}

Symbol : Meaning or definition Unit

A : Open-loop voltage gain of TFT amplifier

$\alpha \quad:$ Frequency dependence factor of flicker noise of TFTs

$\beta \quad$ : Current dependence factor of flicker noise of TFTs

$\mathrm{C}_{d}:$ Equivalent capacitance of pixel detector [Farad]

$\mathrm{C}_{\mathrm{N} 3}$ : Load capacitance of the TFT N3

$\mathrm{C}_{\mathrm{ov}} \quad:$ Gate-to-drain or source overlapping capacitance per unit width

$\mathrm{C}_{\text {probe }}:$ Capacitance of the test probe

[Farad]

$\varepsilon_{\mathrm{i}} \quad$ : Relative dielectric constant of insulator ( 7 for $\mathrm{Si}_{3} \mathrm{~N}_{4}, 4$ for $\mathrm{SiO}_{2}$ )

$\varepsilon_{0} \quad:$ Dielectric constant of vacuum $=8.854 \times 10^{-14}$

$f_{3 \mathrm{~dB}} \quad:$ Cut-off frequency of amplifiers

[F/crn]

[Farad]

$f_{m 3 d B} \quad: \quad$ Measured cut-off frequency of amplifiers

[F/cm]

$\mathrm{g}_{\mathrm{m}} \quad:$ Transconductance of TFTs

[Hz]

$I_{d s} \quad:$ Drain-to-source current of TFTs

$\mathrm{K}_{\mathrm{p}} \quad:=\mu_{\mathrm{fe}} \varepsilon_{\mathrm{o}} \varepsilon_{\mathrm{i}} / \mathrm{t}_{\mathrm{i}}$

L : Channel length of TFTs

$\lambda \quad:$ Channel length modulation coefficient of TFTs

$\mu_{\mathrm{fe}} \quad: \quad$ Field effect electron mobility of TFTs

$\mathrm{R}_{\mathrm{L}} \quad$ : Load resistance of measurement system for diode readout $\left[\mu \mathrm{A} / \mathrm{V}^{2}\right]$

$\mathrm{T}_{\mathrm{i}} \quad$ : Integration time of radiation signal at a pixel

$\mathrm{T}_{\mathrm{d}} \quad$ : Delay time between the incidence of a radiation and readout

[Volt $\left.{ }^{-1}\right]$

$T_{r} \quad: \quad$ Readout time of signal from a pixel

Trise : Rise time of a TFT amplifier

[Ohm]

$\mathrm{t}_{\mathrm{i}} \quad$ : Thickness of gate insulator of TFTs

$\tau \quad$ : Shaping time of a pulse shaping amplifier

$\mathrm{V}_{\mathrm{c}} \quad$ : Bias of the cascode TFT in the prototype poly-Si amplifier 


\begin{tabular}{|c|c|c|}
\hline$V_{d s}$ & : & Drain-to-source bias of TFTs \\
\hline$V_{\text {dd }}$ & : & Main bias of the prototype poly-Si amplifier \\
\hline$V_{\text {gs }}$ & $:$ & Gate-to-source bias of TFTs \\
\hline $\mathrm{V}_{\mathrm{n}}$ & : & Bias of n-ch current source in the prototype poly-Si amplifier \\
\hline $\mathrm{V}_{\mathrm{p}}$ & $:$ & Bias of $\mathrm{p}$-ch current source in the prototype poly-Si amplifier \\
\hline $\mathrm{V}_{\mathrm{r}}$ & $:$ & Reference bias of two gate pulses in two diode readout method \\
\hline $\mathrm{V}_{\mathrm{S}}$ & : & Onset voltage of short channel effect in TFTs \\
\hline$V_{\text {swing }}$ & $:$ & Input voltage swing of the prototype poly-Si amplifier \\
\hline $\mathrm{V}_{\mathrm{T}}$ & : & Threshold voltage of 'TFTs \\
\hline W & & Channel width of TFTs \\
\hline
\end{tabular}




\section{Acknowledgements}

First, I specially want to thank my parents in Korea and the God who gave me a life and materials to keep it alive.

I am very grateful to Victor Perez-Mendez, a wonderful Staff Scientist at LBL and a Professor at the Department of Radiology in UC San Francisco Medical School, for his advice, encouragement, and good sense of humor. Without his support, this work would not have been possible. I also would like to thank my advisor Selig N. Kaplan, Professor at the Department of Nuclear Engineering at UC Berkeley for his careful guidance of my research and for many excellent ideas. I also owe thanks for valuable knowledge and logical ideas from a group of professors at UC Berkeley; L. M. Grossman, D. R. Olander, T. H. Pigford, C. Hu, and R. Gronsky. Their teaching and guidance were not only very useful in my work, but also brought me the love of science and technology.

I also would like to thank my fellow graduate students for their friendship and useful discussions during this research; Ichiro Fujieda, Shafi Qureshi, Tao Jing, Daniel Wildermuth, Hyungkoo Lee and Wansik Hong. Particular thanks go to Dr. John Drewery for making sample devices and helping me to set-up a noise measurement system. I have benefitted enormously from many fine scientists at LBL; Dr. Ali Mireshghi, Dr. Kaarlo Heiskannen, Kai Lee, Donald Landis, and Norman Madden. Also special thanks go to our research collaborators at Xerox PARC, Dr. R. A. Street, Dr. M. Hack, Dr. A. Lewis, and Dr. I-Wei Wu.

Finally, I deeply appreciate the support of two fine and beautiful ladies; Sunja, whom I have always loved and will love forever in my life and Ina, my beloved daughter and the best gift and joy in my life.

This work was supported by the Director, Office of Energy Research, Office of High Energy and Nuclear Physics, Division of High Energy Physics of the U.S. Department of Energy under Contract No. DE-AC03-76SF00098. 


\section{Chapter 1 Introduction}

Amorphous silicon film made by plasma enhanced chemical vapor deposition (PECVD) is a good candidate material for making large area radiation detectors in various imaging applications such as digital $\mathrm{X}$-ray radiography or high energy particle tracking which require good energy resolution and, or good position resolution respectively.

Over the last few years, there have been efforts to use hydrogenated amorphous silicon (a-Si:H) as a detector for ionizing radiation because of its potential for inexpensive and easy fabrication into large area devices, inherent radiation hardness, together with a detection characteristics similar to crystalline silicon.

The most suitable scheme for making position-sensitive radiation detectors out of aSi:H seems to be a pixel detector configuration, which consists of a 2-dimensional array of small pin diodes, because its small detector capacitance reduces system noise. Such an array can be fabricated using the a-Si:H large area deposition and integration process. Also the currently available amorphous or poly-silicon thin-film-transistor (TFT) deposition technique can be used to make pixel readout electronics as well as pixel level front-end amplifiers which can be integrated together with pixel detector array on the same substrate.

In designing such an integrated detection system, analysis of the signal-to-noise ratio is essential for the optimization of detection efficiency, resolution, and timing. Simple modelling to estimate radiation signal and electronic noise of amorphous silicon detectors is required to save time and effort when designing pixel detector systems for various imaging applications.

This thesis describes basic studies on the signal charge collection in a-Si:H detectors and the noise contribution of detectors and front-end TFT amplifiers to the overall system noise. In addition, a-Si:H and poly-Si thin-film-transistors are assessed in a prototype study of readout electronics and pixel level amplifiers. 
Chapter 2 gives a brief but general review of position sensitive radiation detectors and their readout methods. Some necessary background information on hydrogenated amorphous silicon for making radiation detectors is also described.

In Chapter 3, a simple analytical model is derived to estimate the charge collection efficiency in a reverse biased pin diode for various radiation signal and material parameters. Then simulations with the model of charge collection and signal formation from various radiation sources are discussed.

In Chapter 4, in order to estimate the noise contribution from a-Si:H detectors and TFT amplifiers, noise power spectra in the frequency domain are measured and analyzed into various components. Basic properties of a-Si:H and poly-silicon thin-film-transistors such as structure, I-V characteristics and noise components are discussed. The equivalent noise charge expressions of these noise components are obtained from their spectral densities using transfer functions for a simple $C R-(R C)^{n}$ shaping filter. The noise charge of $\mathrm{a}-\mathrm{Si}: \mathrm{H}$ piı detectors measured in a conventional radiation detection system is compared with the sum of calculated noise from the derived expression for various shaping time and biases.

In Chapter 5, after a brief introduction of general 1-dimensional and 2-dimensional detector readout schemes, thin-film-transistor-switch and dicde-switch readout schemes for a-Si:H pixel detectors are discussed. Prototype design and fabrication of pixel-level frontend amplifiers using high temperature poly-Si TFTs are described as well.

Conclusions and final comments are given in Chapter 6. 


\section{Chapter 2 Background}

\subsection{Position-Sensitive Radiation Detectors}

Position-sensitive radiation detectors have become an essential tool in many fields of scientific research and medical imaging. Some examples are: (a) position monitoring and energy spectroscopy of charged particle beams in high energy accelerators, (b) X-ray flux imaging for digital radiography, and auto-radiography to study DNA crystalline structure of bio-specimen, (c) positron emission tomography and single photon imaging in nuclear medicine to obta 1 the distribution of radio-isotopes in a body, (d) X-ray diffraction study and $X$-ray or synchrotron radiation microscopy, (e) astronomical telescope to measure the intensity and distribution of cosmic radiations, and (f) neutron radiography for nondestructive industrial applications, and so on.

Depending on the specific radiation types and the required detection condition or restrictions such as detector size, fast tirning and real time imaging capability, good spatial or spectral resolution, and radiation hardness, many different types of radiation detectors have been used; $\mathrm{X}$-ray films, multiwire gas detectors, drift chambers, scintillators with photomultiplier tubes, and various semiconductor detectors. Some examples are given below.

(1) X-ray films: They are the most common 2-d imaging devices but limited in application because of riariow range of sensitivity to radiations and moie importantly lack of real-time imaging capability.

(2) Gas detecitors : For the position detection of charged particles, gas chambers with multiwire anode and cathode strips on a pad are used. Two recent examples include a position monitoring system for an anti-proton beam developed at CERN [1] and tracking detectors for many charged particles with multiplicity produced from a heavy ion collision experiment built at BNL.[2] 
(3) Scintillators : Thin plates of various scintillators, organic or inorganic, converts the energetic particles or $\mathrm{X}$-rays into visible light and then an array of photomultiplier tubes or semiconducting photodiodes detects the photons. Over the last decade, a major improvement in spatial resolution in X-ray imaging was achieved by scintillating optical fiber techniques [3] and micro-channel plate technique [4] by narrowing the angle of light emission. X-ray image intensifiers with TV cameras are often used in X-ray digital imaging.[5]

(4) Semiconductor detectors : Rapidly advancing technology of semiconductor microelectronics and the availability of high purity semiconductor wafers are enabling the construction of precision position sensitive radiation detectors. Basically all the semiconductor detectors adapt the same principle that the generated charges are drifted toward the electrodes under the applied electric field in a depleted region and induce an external current pulse.

Three of the most interesting position sensitive silicon detectors are (a) microstrip detectors [6] with charge collection on individual strip electrodes, which are at present the highest spatial resolution detectors and are commercially available, (b) semiconductor drift chambers [7] which employ a principle similar to that of gas drift chambers, and (c) charge coupled devices (CCD) which transfer collected charges from cell to cell by peristaltic potential variation. They have been employed successfully in recent physics experiments with spatial resolution as high as $5 \mu \mathrm{m}$ in one-dimension.[8] Since silicon has a low interaction rate with soft $\mathrm{X}$-rays, its coupling with scintillators enhances the efficiency of medical X-ray imaging system. Other semiconductors like $\mathrm{Ge}, \mathrm{HgI}_{2}, \mathrm{CdTe}$ are also often used to detect $\mathrm{X}$-rays directly. 


\subsubsection{Two-Dimensional Silicon Pixel Detectors}

Fig. 2.1 shows a schematic top-view of a 1-dimensional linear array and of a 2dimensional pixel array of radiation detectors. Sometimes 1-dimensional (linear or strip) arrays of detectors are sufficient but 2-dimensional detectors will be necessary to reduce the image scanning time in most radiological applications and to avoid ambiguities arising from multiple coincident events in particle physics experiments. Though 2-dimensional imaging can be accomplished using double-sided Si strip detectors with crossed or inclined strips, or segmented Si drift chambers,[9] they would not be as efficient as a genuine 2. dimensional array, which we call a pixel detector. The term 'pixel' is an abbreviation of the word 'picture element,' referring to the smallest discernible element in image processing devices such as a CCD camera or a color television set.

Recently new architectural designs of pixel detectors have been strongly encouraged and enabled by improvements in silicon VLSI manufacturing technology. There are two types of pixel detectors, monolithic and hybrid type devices, depending on whether detectors and electronics are integrated in a single chip or made in two separated chips and bonded together later.

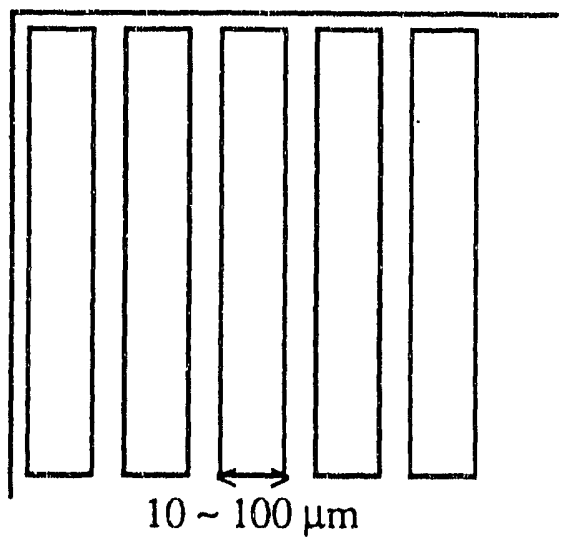

(a)

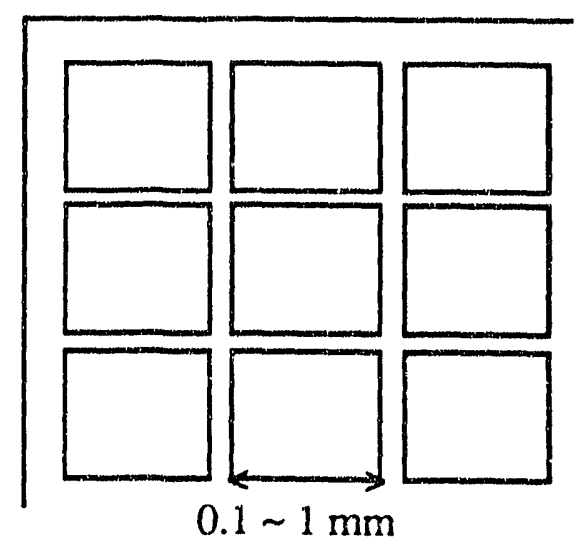

(b)

Fig. 2.1 Top views of a linear and a pixel array of a-Si:H radiation detectors. 
(1) Monolithic detectors:

CCDs are a good example of monolithic pixel devices which have been originally developed for optical imaging in television video cameras and were first used as a radiation detector by Damerell et al.[8] However CCDs have drawbacks, such as a small signal because of the thin sensitive layer thickness (typically $5 \sim 10 \mu \mathrm{m}$ ) and an inherent slow readout speed. Holland et al.[10] have recently suggested and developed monolithic silicon detectors by making a thick pin diode on one side and transistor electronics on the other side of a high-resistivity silicon wafer.

(2) Hybrid detectors:

Hybrid type pixel detectors have been initially employed to image infrared radiation for astronomy using 'flip-chip' technology, which means that the detector chip and electronics chip are fabricated separately and bonded, pixel-to-pixel, together using indium bump bonding [11] or gold ball-conductive epoxy bonding [12] as shown in Fig. 2.2. The hybrid types of detectors have a strong advantage in that detectors and electronics can be separately optimized using appropriate material and processing technology. Therefore they can have nearly $100 \%$ coverage of the sensitive area by detectors and fast readout speeds. However the high fabrication cost and uncertainty of yield in large area devices are major problems.

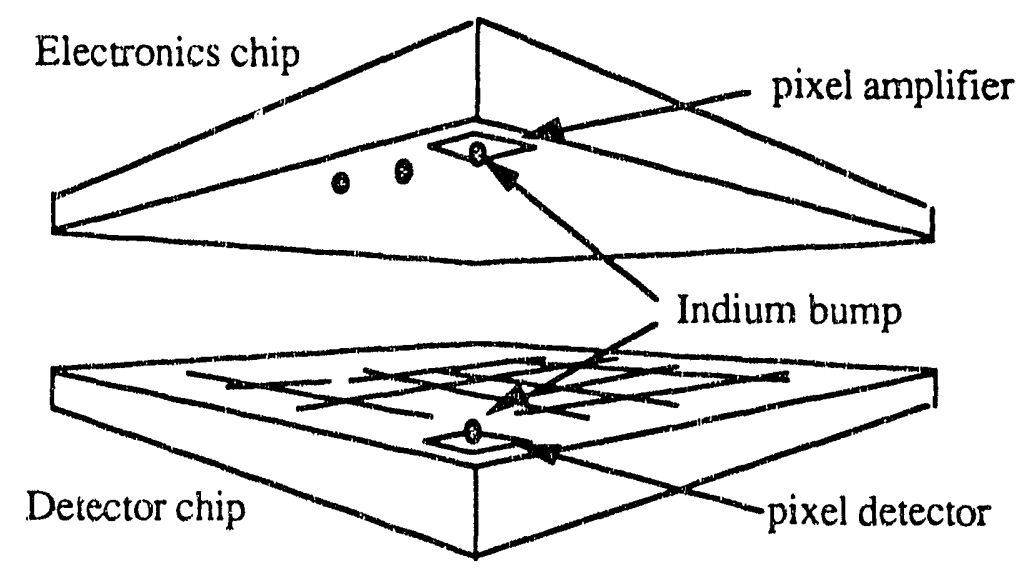

Fig. 2.2 A schematic diagram of indium bump technique. 


\subsection{Readout Electronics}

The signal processing system applied to a single radiation detector usually consists of a preamplifier, a shaping amplifier and a single-channel analyzer (SCA) for counting or multichannel analyzer (MCA) for energy spectroscopy as shown in Fig. 2.3.

The preamplifier amplifies the detector signal, which is usually very small, for further processing. Usually in a semiconductor detector, a charge-sensitive preamplifier is used to yield an output voltage pulse which is not only directly proportional to the signal charge but also insensitive to the total input capacitances from the detector and amplifier input node, including any stray capacitance.[13] The preamplifier should also have a low output impedance to drive subsequent electronic components, and it should be located as close as possible to the detector to minimize noise-producing stray capacitance.

The pulse-shaping amplifier often amplifies the output pulse of a charge-sensitive preamplifier further, but its main function is to shape the output pulse of the preamplifier, which is generally designed as a current integrator producing a step pulse or a long-tailed pulse due to slow decay of the charge in the feedback capacitor through a high resistance ( $100 \mathrm{M} \Omega$ ) in parallel with the capacitor. The shaping amplifier produces a short pulse, avoiding pulse pile-up of a subsequent signal on the tail, especially in the case of high

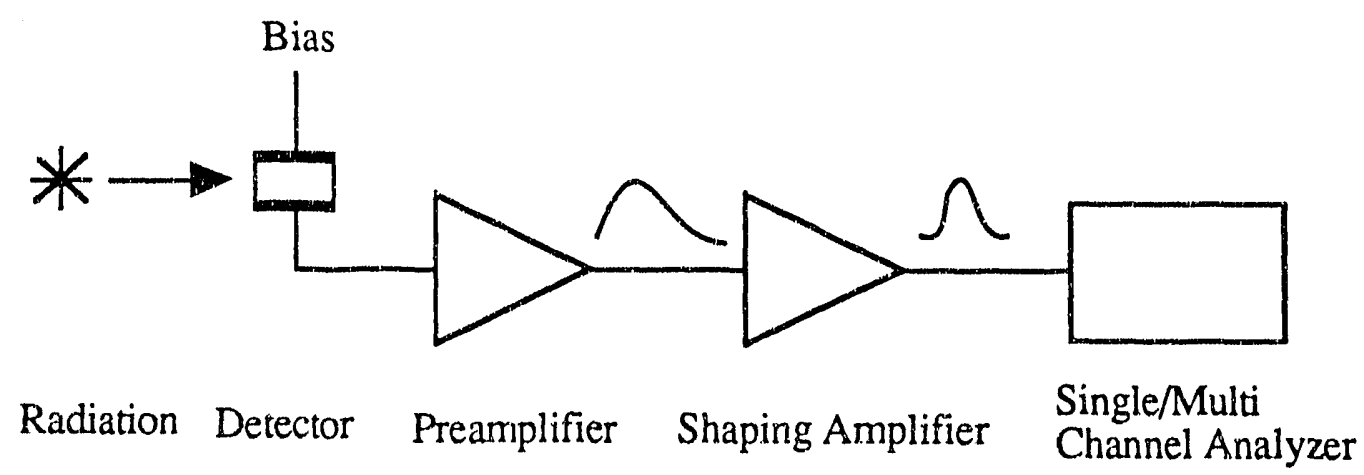

Fig. 2.3 A schematic diagram of signal pulse processing electronics for single detectors. 
detection rates. Shaping also functions to achieve the best signal-to-noise ratio by eliminating electronic noise of low and high frequencies compared to the characteristic frequency of the signal pulse. In its simplest form, it consists of a high pass and low pass filter network such as CR-RC. But there can be many types of pulse shaping depending on the specific requirement. [14]

A single-channel analyzer is composed of a pulse discriminator and a counter. It counts the number of signal pulses which have higher amplitude than a certain discriminating level. A multichannel analyzer is basically a combination of an analog-todigital (ADC) converter and an addressable memory and it displays the distribution of pulse counts in a digitized scale of the pulse height, called channels.

Besides the typical pulse processing electronics mentioned, another important requirement in 1-dimensional and 2-dimensional position sensitive detectors, is the signal readout electronics because there is an array of many detectors. The signal readout is a method for sending detector signals to the pulse processing electronics located externally. It plays an important role in determining the time and position of the radiation pulse.

\subsubsection{Readout Schemes for 1-D Detector Array}

Prior to developments in integrated circuit technology, 1-dimensional detectors such as a multiwire proportional counter or silicon strip detectors used a pair of distributed amplifiers to determine position by several interpolating readout methods.[15] Two typical examples are:

(a) The delay line method: where the cathode wires or strips are connected at uniform spacing to a helical coil of delay line, and the position is determined by the difference of signal arrival times at two amplifiers. 
(b) The resistive electrode method : where the wires or strips are connected to a resistive electrode and the position is determined either by the ratio of charge division or by the difference of pulse rise time measured by two amplifiers.

A schematic of these methods is shown in Fig. 2.4(a).

Another 1-d readout scheme is to use separate amplifiers connected to every strip as seen in Fig. 2.4(b). While requiring many amplifiers, this scheme is much better for readout speed and signal multiplicity because every detector signal can be processed at the same time and independently. Recent developments in silicon analog integrated circuit technology and fabrication process such as wire bonding, enable the making of compact, reliable, low cost, and easily usable readout chips containing many amplifiers.[16] For example a silicon vertex detector (SVX) chip developed at Lawrence Berkeley Laboratory, [17] has low noise signal processing electronics for 128 input channels. It was developed for the hadron collider detector at Fermi Laboratory (CDF) and has been successfully tested in the stationary target experiments.[18]

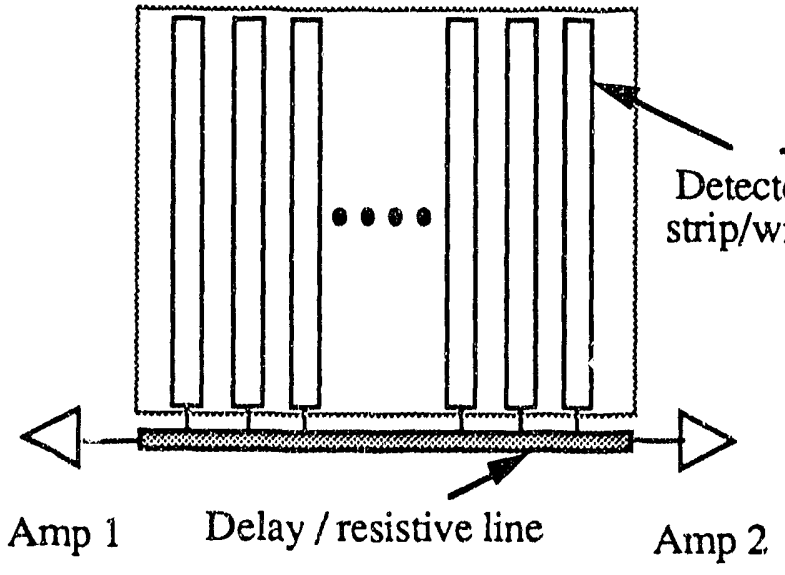

(a)

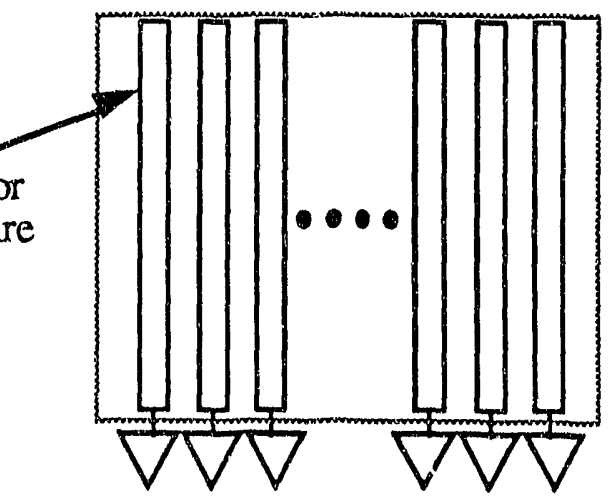

Amplifiers

(b)

Fig. 2.4 Schematics of readout schemes from 1-dimensional detector array (a) delay line or resistive line readout scheme using two amplifiers (b) readout of signals from every detector strip or wire using the same number of amplifiers. 


\subsubsection{Readout Schemes for 2-D Detector Array}

2-dimensional detectors, by nature, require more elaborate readout schemes. Depending on various factors such as detector types (CCD, pixel detector, etc.), position sensing or image sensing, and photo-like or movie-like detection, different schemes should be employed. However decision of a readout scheme is a process of compromise between readout speed and the complexity of the electronics, such as the number of interconnections and the number of signal processing amplifiers. For very large area detectors with a number of pixels $>10^{6}$, the whole detector system can be assembled by putting many sets of individual 2-d detectors side by side. In this case, each set may be processed in parallel in time.

In this section the discussion is limited only to 2 -d pixel detectors because of interest in its application to hydrogenated amorphous silicon.

\section{(1) Every Pixel Readout Method:}

It would be ideal if every pixel were connected to its own pulse processing electronics through its own data routing line and all the 2-d data were read at once.

A detector employing hexagonally-shaped pixels [19] has used this method. Every pixel sends signals through its own data line to a linear array of amplifier chips located either at the top or bottom edge of the detector array. This method gives very fast readout speed but it requires a large number of pulse processing components. Also, since the data lines are numerous and become so closely spaced as the pixel size becomes smaller, it rapidly reaches a practical size limit. Hence a more realistic and smart readout scheme is necessary. 


\section{(2) X-Y Readout Method:}

This methods involves reading the signal from common $\mathrm{x}$ - and $\mathrm{y}$-data lines at the same time as shown in Fig. 2.5(a). Therefore it can give a very high readout speed, but produces an ambiguity in the case of multiple hits during one resolution time.

A detector called the XYW detector, has been designed for a 2- $\mathrm{d}$ pixel array by Diericky [20] to give a very high readout speed. Each pixel consists of a pixel diode, a gated charge-sensitive amplifier, and a two-transistor output stages. The output of the charge sensitive amplifier is connected to a common gate of the two transistors and modulates the drain-to-source current simultaneously. While the drain electrodes of the transistors are connected to a common bias, the source electrodes are connected to a common row $(x)$ and column ( $y$ ) data lines, respectively. Two external signal processing circuits, one for an $\mathrm{x}$ data set and one for a $\mathrm{y}$ data set, compare inputs (sums of signals from pixels in each row and column respectively) with a given threshold level and produce a digital address of the row and column connected to any pixel producing a radiation signal. (3) Line-Scanning Method :

This method involves scanning the data of pixel diodes line by line in sequence so the $\mathrm{x}$ coordinate is determined by the time of the gate pulse and the $\mathrm{y}$ coordinate is given by a column index, as shown in Fig. 2.5(b). Therefore its readout speed is limited by the number of rows. This system often requires temporary data storage at the pixel level due to the relatively long readout time compared to the other methods, above. However, it has no ambiguity in the position of radiation which is important in particle physics applications, and it also produces a real-time imaging which is often required in medical applications.

A line-scanning readout, the Eyeball-array,[21] has been developed at Xerox PARC for an a-Si:H photodiode array. Each pixel consists of a photodiode and a readout transistor made of a-Si:H. Details of its description will be given in the next section. A similar line scanning readout method using two transistors has been suggested by Parker [22] for a proposed silicon pixel detector array. 
Pixel

Gate line

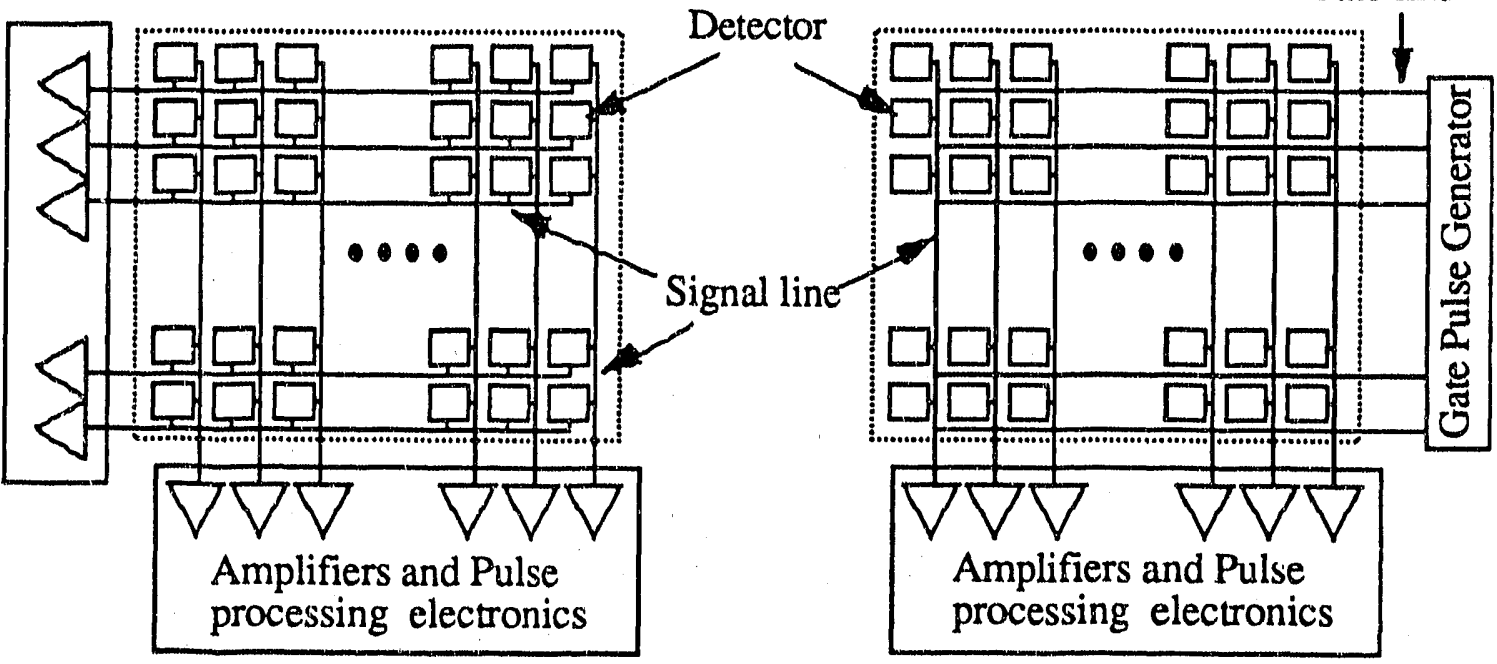

(a)

(b)

Fig. 2.5 Schematic diagrams of readout methods for a 2-dimensional pixel detector array (a) $x-y$ readout method (b) line scanning method.

\subsection{Hydrogenated Amorphous Silicon (a-Si:H)}

Hydrogenated amorphous silicon has a relatively long application history as a solar cell and a photo-sensor for optical imaging such as electron-photography (Xerography) [23] and a facsimile head.[24] Schottky and pin diodes made of this material had been first suggested for use as ionizing radiation detectors by Kaplan et al.[25] Some other groups have tested this material as X-ray sensors with a phosphor such as $\mathrm{CdWO}_{4}$.[26] They have emphasized that this material can be deposited onto large surfaces and produce a relatively high signal from interactions with ionizing radiation. Another potential benefit is its inherenc high radiation resistance because of its noncrystalline structure.[27] 


\subsubsection{Production Methods}

Hydrogenated amorphous silicon (a-Si:H) is an alloy of silicon and hydrogen which is deposited on a substrate (glass, quartz, plastic or metal) in a thin film from the gaseous state. Amorphous silicon has been known for a long time but it has only attracted attention as an electronic material since doping to produce p-type and n-type elements was first demonstrated by Spear and LeComber in 1975,[28] who showed that by adding dopant gases such as diborane $\left(\mathrm{B}_{2} \mathrm{H}_{6}\right)$ or phospine $\left(\mathrm{PH}_{3}\right)$ to silane gas $\left(\mathrm{SiH}_{4}\right), \mathrm{p}$ - and $\mathrm{n}$ type semiconductor material would be produced.

Out of the various methods of production from the precursor-gas state : sputtering, chemical vapor deposition (CVD), PECVD, photo-CVD, and so on, The PECVD, often called rf-glow discharge, is known to produce best quality semiconductor material with lowest defect density.

In the PECVD method, silane gas, sometimes diluted with hydrogen, is fed into a vacuum chamber through a control valve and decomposed in a glow discharge by capacitively or inductively coupled rf power into $\mathrm{SiH}_{\mathrm{n}}{ }^{-}(\mathrm{n}=1 \sim 3)$ and $\mathrm{H}^{+}$and forms a weakly ionized plasma. $\mathrm{SiH}_{\mathrm{n}}{ }^{-}$ions, precursors, diffuse onto a substrate and form a $\mathrm{Si}-\mathrm{Si}$ network by chemical reactions assisted by the heat. Heat is transferred from the filament underneath the substrate by controlling its temperature. During the deposition process hydrogen gas is evolved from the surface and is removed by the vacuum pump together with the excess gas. Fig. 2.6 shows a schematic diagram of LBL PECVD system.

Deposition conditions such as gas flow rate, gas pressure, if power density and substrate temperature, play a critical role in determining the quality of films and growth rate. Optimization of the deposition condition requires long period of trials and errors. A typical deposition condition for LBL PECVD system at $85 \mathrm{MHz}$ of $\mathrm{rf}$ glow discharge frequency is summarized in Table 2.1 where $\mathrm{sccm}$ stands for standard cubic $\mathrm{cm}$. 


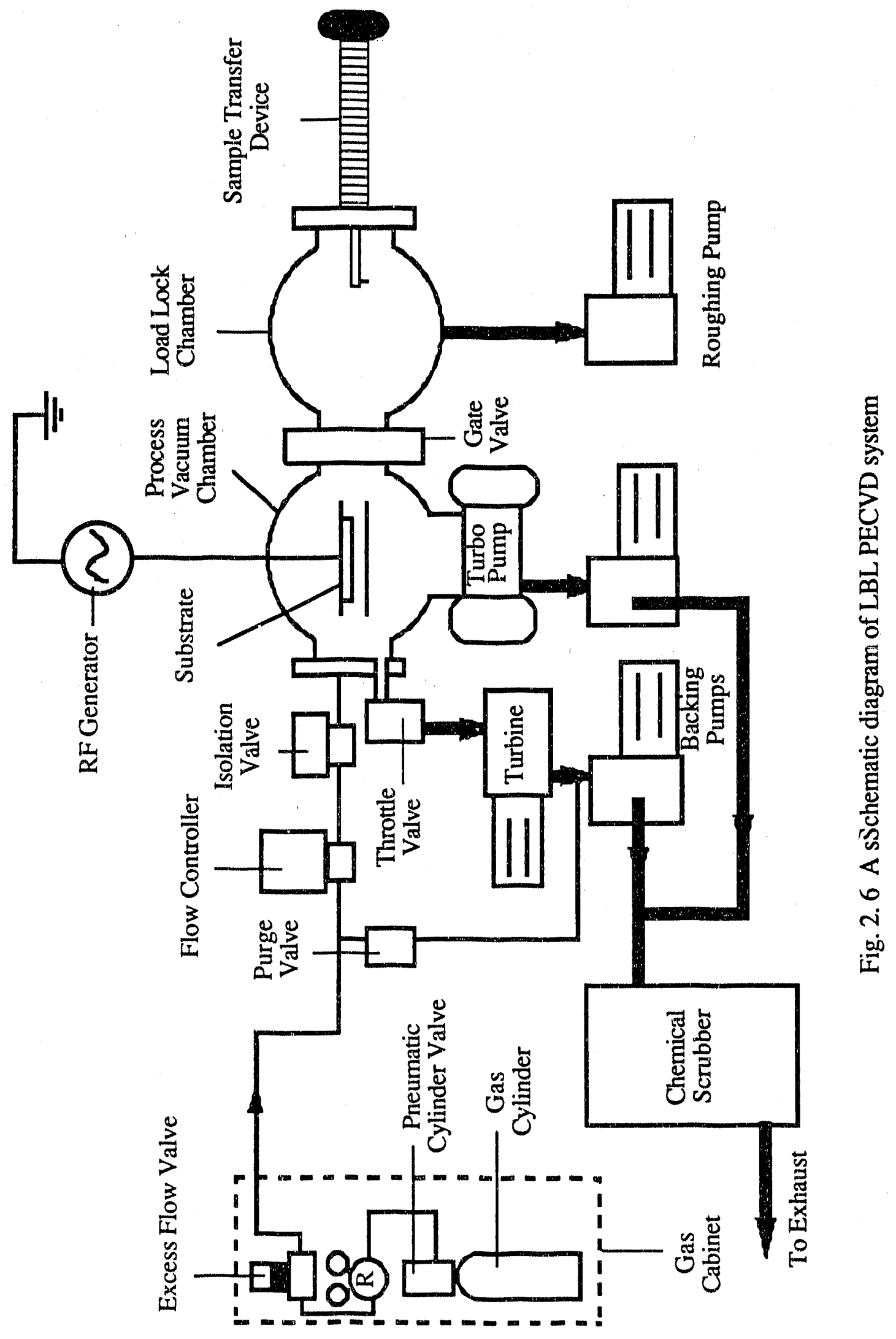


Table. 2.1 Deposition parameters of LBL PECVD system

\begin{tabular}{|c||c|c|c|}
\hline Layer type & n-layer & i-layer & p-layer \\
\hline Gases \& flow rate & $\mathrm{SiH}_{4}: 40 \mathrm{sccr}$ & $\mathrm{SiH}_{4}: 40 \mathrm{sccm}$ & $\mathrm{SiH}_{4}: 40 \mathrm{sccm}$ \\
& $\mathrm{PH}_{3}: 6 \mathrm{sccm}$ & & $\begin{array}{c}\mathrm{B}_{2} \mathrm{H}_{6}: 15 \mathrm{sccm} \\
\mathrm{CH}_{4}: 90 \mathrm{sccm}\end{array}$ \\
\hline Heater temperature & $320^{\circ} \mathrm{C}$ & $320^{\circ} \mathrm{C}$ & $250{ }^{\circ} \mathrm{C}$ \\
\hline $\mathrm{RF}$ power & $5 \mathrm{Watt} / \mathrm{cm}^{2}$ & $7 \mathrm{Watt} / \mathrm{cm}^{2}$ & $5 \mathrm{Watt} / \mathrm{cm}^{2}$ \\
\hline Growth rate & $27 \mathrm{~nm} / \mathrm{min}$ & $38 \mathrm{~nm} / \mathrm{min}$ & $27 \mathrm{~nm} / \mathrm{min}$ \\
\hline
\end{tabular}

\subsubsection{Basic Properties}

Atomic silicon has four valence electrons and its most stable form is a crystalline diamond structure. Amorphous silicon is a random network of silicon atoms, a schematic of which is shown in Fig. 2.7. The optimum coordination number to get the minimum freeenergy of the ideal random network system has been theoretically calculated to be $\leq$ 2.4.[29] Because of this there are inherent structural defects such as dangling bonds and voids which act as trapping and recombination centers for electrons and holes.

Hydrogenation makes a better material than pure amorphous silicon in terms of the mechanical and electronic properties because the hydrogen atoms tend to terminate dangling bonds and therefore reduce the defect states from $\sim 10^{17}$ to $\sim 10^{15} \mathrm{~cm}^{-3}$ in the gap between the conduction and valence bands. Atomic hydrogen concentrations of $\sim 10 \%$ of the amorphous silicon density is known to make a device quality material.[30] Extra hydrogen atoms left after compensation of dangling bonds, remain in atomic or gas molecular form in the silicon matrix and voids. They make bubbles or diffuse out of the material depending on the temperature and hydrogen partial pressure of the bulk material and of the environment. When the atomic concentration of hydrogen is less than $5 \%$ micro-crystalline 
structures develop.[31] Table 2.2 is a comparison of fundamental material properties of crystalline silicon and hydrogenated amorphous silicon.

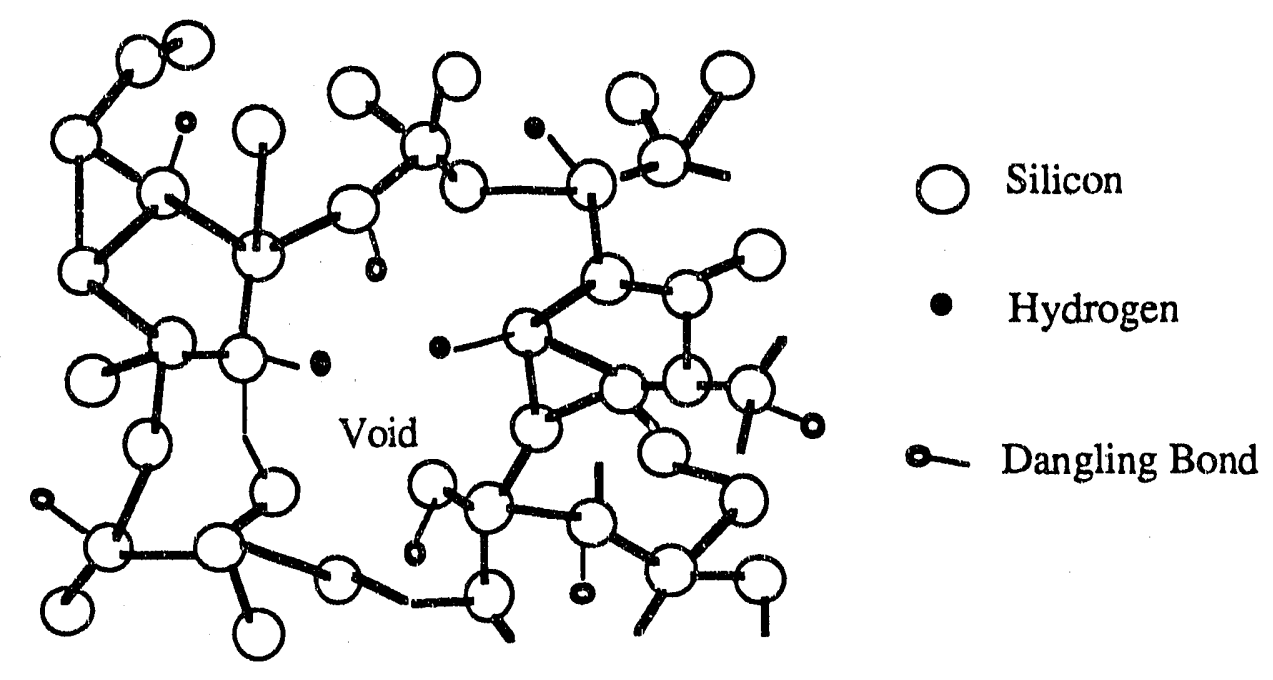

Fig. 2.7 A schematic structure of a-Si:H showing dangling bonds and a void.

Table 2.2 Material properties of crystalline silicon and hydrogenated amorphous silicon

\begin{tabular}{|c||c|c|}
\hline Properties & Crystalline Si & a-Si:H \\
\hline \hline Structure & Diamond & random network \\
\hline Interatomic distance $(\AA)$ & 2.35 & $2.35 \pm 0.07$ \\
\hline First bond angle $\left(^{\circ}\right)$ & 109.47 & $109 \pm 10$ \\
\hline Density $\left(\mathrm{gr}^{\circ} / \mathrm{cm}^{3}\right)$ & 2.3 & $\sim 2.25$ \\
\hline Dielectric constant & 12.0 & $\sim 11.8$ \\
\hline Resistivity $(\Omega \mathrm{cm})$ & $<10^{5}$ & $>10^{9}$ \\
\hline Electron mobility $\left(\mathrm{cm}^{2} / \mathrm{Vsec}\right)$ & 1350 & $1 \sim 2$ \\
\hline Hole mobility $\left(\mathrm{cm}^{2} / \mathrm{Vsec}\right)$ & 480 & $0.004 \sim 0.007$ \\
\hline Band gap energy $(\mathrm{eV})$ & 1.12 (indirect) & $1.7 \sim 1.9$ (direct) \\
\hline
\end{tabular}




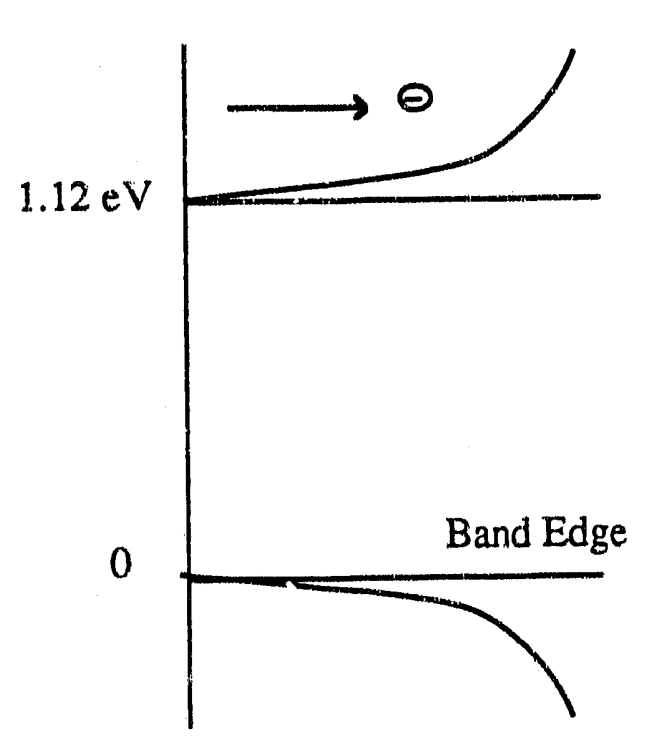

(a) Crystalline- $\mathrm{Si}$

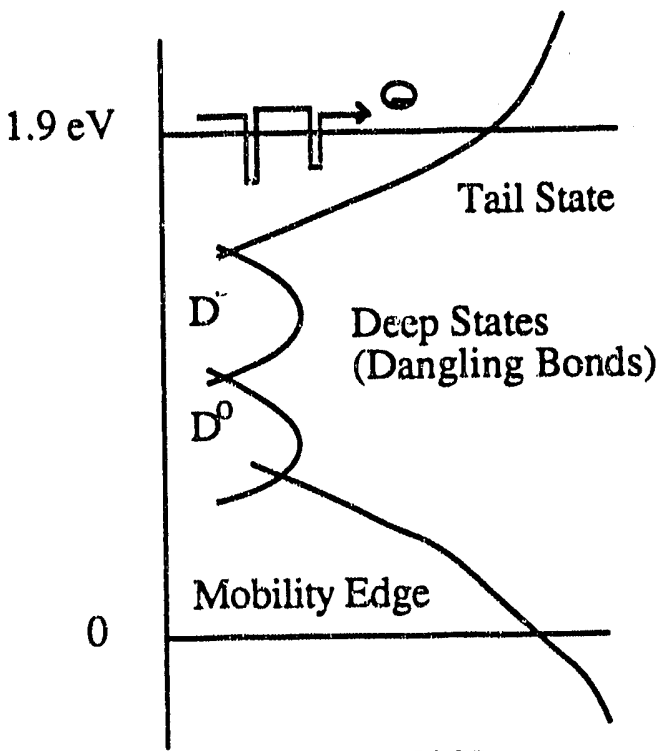

(b) a-Si:H

Fig. 2.8 Schematic band structures of (a) crystalline and (b) amorphous silicon.

Schematics of band structures of crystalline silicon and amorphous silicon are shown in Fig. 2.8. Conduction and valence bands are not well defined in amorphous silicon. Each has a deeply penetrating band tail, called the Urbach edge, [32] into the forbidden gap due to lack of long range order in the random network, and there is a high density of gap states, mostly originated from dangling bonds.

In the band tail states, the density of state becomes low so that the electron wave function does not overlap between the states. Hence electrons or holes which are mostly located in the tail states can not move as freely as in the conduction or valence band and the transport of electrons or holes is characterized by ranges in values of mobilities depending on the energy state. In contrast to crystalline silicon, the observed average mobility, often called the drift mobility of amorphous silicon around room temperature, increases as the temperature is raised because of excitation of trapped electrons in the tails states to higher conduction states. The observed mobilities are typically $\sim 1 \mathrm{~cm}^{2} / \mathrm{Vsec}$ and $\sim 0.004$ $\mathrm{cm}^{2} / \mathrm{Vsec}$ for electrons and holes respectively at room temperature. Because of the 
asymmetrical density of states with more states in the lower half of the gap, the average drift mobility of holes is much lower than that of electrons. Also the hole transport is dispersive and is governed by a hopping mechanism even at room temperature. At very low temperatures, transport of both carriers is dominated by a hopping mechanism rather than drift. Hopping is a conduction mechanism in which carriers jump from a localized tail states to a neighboring tails state under the influence of the electric field. The transition temperature between drift and hopping mechanisms for electrons and holes are typically $150^{\circ} \mathrm{K}$ and $\sim 360^{\circ} \mathrm{K}$ respectively. $[33,34]$

The mobility edge in non-crystalline solids suggested by Mott [35] is a conveniently defined concept to represent that all the electrons having their kinetic energy above the conduction band mobility edge, called free electrons, are assumed to have a constant mobility often called an extended drift mobility, and all the electrons with energy below the mobility edge, called trapped electrons, have zero mobility, and similarly for the holes. Then there is an equilibrium of trapping and detrapping process of free carriers and trapped carriers at any temperature.

The average energy state of dangling bonds is located in the middle of the forbidden gap between the conduction and valence bands and the density of states has a broad peak which is often simulated as a gaussian shape with a width of a few tenth of eV. If a dangling bond captures an electron, the energy states of these two electrons deviates by 0.2 $\sim 0.3 \mathrm{eV}$. This is the repulsion between two electrons with opposite spins and is called 'the correlation energy'.[36] Electronically a dangling bond can have three states; (a) singly occupied ( $\left.\mathrm{D}^{0}\right)$, (b) doubly occupied( $\left.\mathrm{D}^{-}\right)$and $(c)$ empty $\left(\mathrm{D}^{+}\right)$. When the dangling bond is occupied by a single electron, it is neutral. Fig. 2.4 (b) shows $\mathrm{D}^{\circ}$ and $\mathrm{D}^{-}$states. We can consider $D^{0}$ states to be filled and $D^{-}$states empty. Therefore the Fermi energy level is located in the middle of these two peak. The density of dangling bonds is normally measured by an electron spin resonance technique.[37] 
The Staebler-Wronski effect,[38] the generation of metastable defect states from light soaking, electric stress, and aging breaking weak silicon bonds into dangling bonds is still the main way in which the device quality is degraded with time.

\section{4 a-Si:H Pixel Detector Structures}

A-Si:H radiation detector structure is a reverse biased pin diode which is similar to that of solar cells. A detector consists of a metallic layer and a thin heavily doped p layer ( $30 \mathrm{~nm}$ ), followed by an intrinsic layer which forms the bulk of the diode, then a thin heavily doped $\mathrm{n}$ layer $(\sim 30 \mathrm{~nm})$ and finally a thin metallic layer. Chromium or palladium are the usual metals that are used for the electrical contact. The $\mathrm{p}$ and $\mathrm{n}$ layers are blocking layers to minimize the reverse current. The bulk $i$ layer acts as the main region where the incident radiation interacts and produces the secondary electron-hole pairs. The i-layer must be thick enough to produce enough electron-hole pairs to give a detectable signal.

For use as a radiation detector two possible schemes may be implemented :

(a) Indirect detection: A thin $(1 \sim 5 \mu \mathrm{m})$ pin diode is coupled to a thick $(0.3 \sim 1 \mu \mathrm{m})$ CsI(TI) scintillator for $\mathrm{x}$-ray or single particle detection.

(b) Direct detection : A thick $(50 \sim 70 \mu \mathrm{m})$ pin diode is used for direct detection of single particles especially minimum ionizing particles.

Fig. 2.9 (a) and (b) show cross sectional conceptual views of pixel elements which integrate pixel amplifier and readout electronics on a glass or quartz substrate with deposited a-Si:H layers. 


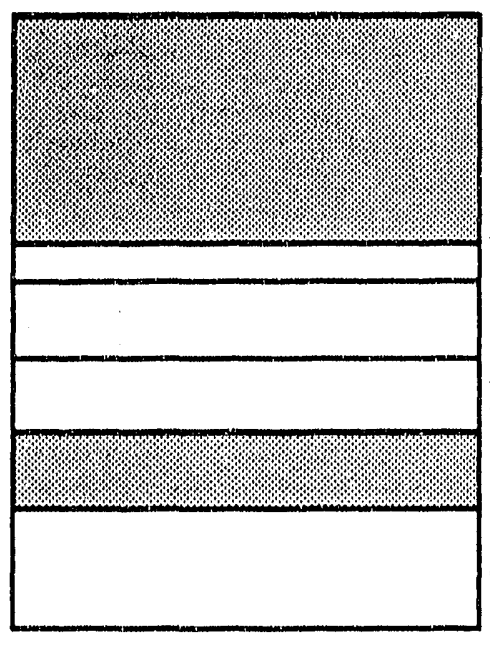

(a)

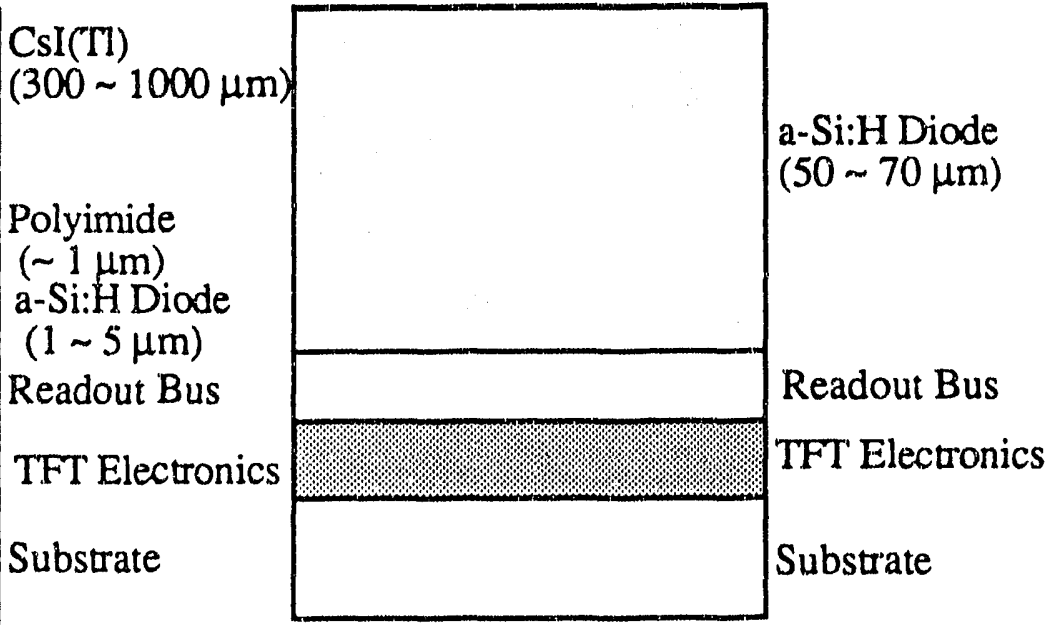

(b)

Fig. 2.9 Cross-sectional view of a single pixel ; (a) indirect detection scheme using a scintillating layer coupled to a thin a-Si:H photodiode and (b) direct detection scheme using a thick a-Si:H diode.

\subsubsection{Thin Detector Coupled to Scintillating CsI(TI) Layer}

In this configuration, the scintillating layer converts the energy deposited by $\mathrm{x}$-rays or single particles into visible light; the thin a-Si:H pin diode absorbs the scintillation light and generates electron-hole pairs. For the effective transmission of the scintillation light, a thin metal layer $(<100 \mathrm{~nm}$ of $\mathrm{Cr}$ ) or a transparent conducting layer such as indium-tin-oxide (ITO) may be used for the top contact of the diode in Fig. 2.9 (a).

Among various scintillating materials, $\mathrm{CsI}(\mathrm{Tl})$ appears the most promising for the following reasons :

(a) Large-area deposition : It is readily deposited by vacuum evaporation at low deposition substrate temperature [39] in the range of $50 \sim 250^{\circ} \mathrm{C}$. This allows for the possibility of direct evaporation on to an a-Si:H photo diode layer without degrading the a-Si:H which start to degrade at $250^{\circ} \mathrm{C}$. The $\mathrm{CsI}$ adheres well to roughened glass or roughened $\mathrm{Al}$. However in order to deposit it with good bonding on ITO coated a-Si:H it was found necessary to deposit an intermediate layer of $\sim 1 \mu \mathrm{m}$ thick polyimide which is transparent to 
the scintillation light.[40] The polyimide is formed by spin coating and is baked at $200^{\circ} \mathrm{C}$. The details of the evaporation conditions for the CsI have been reported. [41]

(b) Good spatial resolution : Depending on the deposition conditions, it forms columnar structures [42] which limit the light diffusion sideways and allows high spatial resolution. $100 \mu \mathrm{m}$ of spatial resolution has been demonstrated from $1 \mathrm{~mm}$ thick $\mathrm{CsI}(\mathrm{Tl})$ by $17 \mathrm{keV} \mathrm{X}$ ray which was a factor of 2 higher than that of the same thickness $\mathrm{Gd}_{2} \mathrm{O}_{2} \mathrm{~S}$, a commonly used scintillator.[41]

(c) Light properties : After interacting with the incident radiation, $\mathrm{CsI}(\mathrm{Tl})$ emits a spectrum of light with decay times of 1100 and $600 \mathrm{nsec}$. The emitted light is in the visible and matches well with the absorption response of a-Si:H as shown in Fig. 2.10 which compare the light spectrum with other CsI materials.[43] The light yield is 50,000 visible photons per $1 \mathrm{MeV}$ energy deposit, which is among the highest light output of any known scintillator.[44] CsI(TI) layer 300 $1000 \mu \mathrm{m}$ thick will produce 12,000 40,000 e-h pairs in a thin a-Si:H diode for minimum ionizing particles after correcting for light scattering and transmission losses.[41]

The main disadvantage of $\mathrm{CsI}(\mathrm{Tl})$ scintillators is that their resistance to radiation damage by energetic particles (fast neutrons or high energy protons) and by gamma rays is not as high as that of a-Si:H. Perez-Mendez et al.[45] have shown that a-Si:H is extremely radiation resistant and is not damaged by fast neutron fluxes $>10^{14} \mathrm{n} / \mathrm{cm}^{2}$. However, because the $\operatorname{CsI}(\mathrm{Tl})$ is deposited in thin layers, the degradation due to radiation which is largely due to attenuation of the light transmission is less than that of thick crystals. For example, for $\mathrm{a} \sim 100 \mu \mathrm{m}$ thick deposition the signal from gamma rays emitted by $\mathrm{Co}^{60}$ dropped by half after an irradiation dose of $10^{6} \mathrm{rad}$ of the same gamma rays.[41] By comparison with a $1 \mathrm{~mm}$ crystalline $\mathrm{CsI}(\mathrm{Tl})$ detector, the signal dropped to half at $10^{4}$ rad.[46]

$\operatorname{CsI}(\mathrm{Na})$ is more radiation resistant than $\mathrm{CsI}(\mathrm{Tl})$ [47] and it has a short lived $\sim 20$ nsec component but it is hygroscopic and emits less light (38,000 photon/MeV).[39] Other 
scintillators, such as cadmium tungstate, gadolinium oxysulfide, gadolinium silicate etc., which can be deposited in large areas may also prove applicable to the indirect detection method.

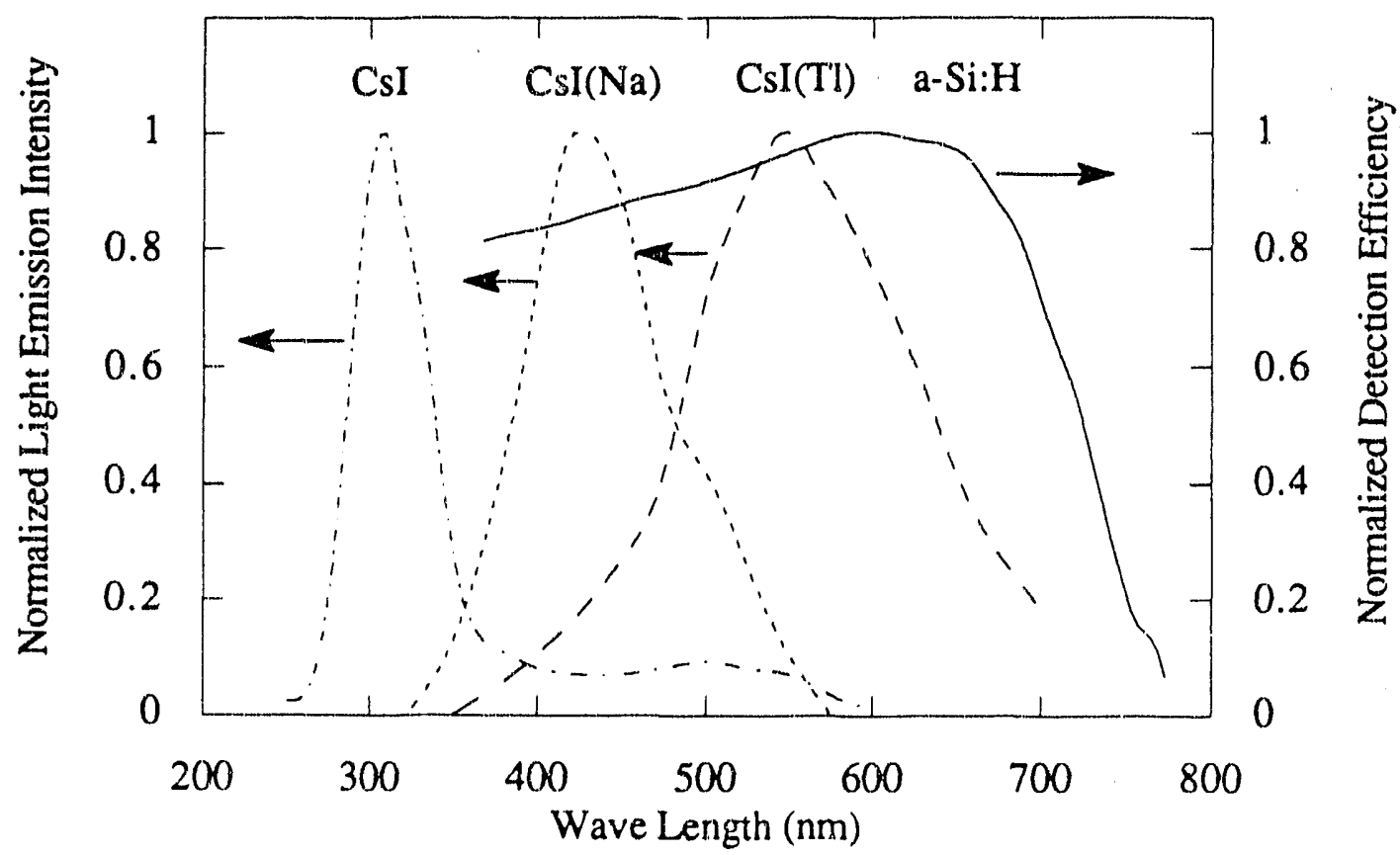

Fig. 2.10 Scintillation light spectra of pure C.sI, CsI(Na) and CsI(Tl) and detection efficiency of a-Si:H photodiodes.

\subsubsection{Thick Detector for Direct Detection of Ionizing Radiations}

In high energy physics experiments in electron-positron collider or protonantiproton collider accelerator, detection of minimum ionizing particles is the major concern. Those particles such as fast electrons or other elementary particles produce a uniform distribution of electron-hole pairs as they pass through the silicon.

In order to detect minimum ionizing particles direwily with a-\$i.h diodes, the $i$ region containing the electric field must be thick enough to produce an adequate signal above noise. The raudiation signal is basically proportional to the energy absorbed in the 
bulk of detector where the electric field is not zero. And the generated charges will drift and be collected. There are some material problems in making thick radiation detectors using the conventional PECVD. As the a-Si:H film becomes thicker, compressive stress develops and either cracks are generated in the a-Si:H layer or the substrate bends.

In order for the electric field to penetrate into the i region, it must be fully depleted. A very large bias is required for this due to the large density of space charges which are mostly from the ionized dangling bonds.

However recently there has been improvements in the deposition technique. For instance, production of $50 \sim 100 \mu \mathrm{m}$ pin diodes with dangling bond density $\leq 2 \times 10^{15}$ $/ \mathrm{cm}^{3}$ deposited in a $110 \mathrm{MHz}$ PECVD machine at a deposition rate of $2 \sim 3 \mu \mathrm{m} /$ hour has been reported.[48] 


\section{References}

[1] M. van dien Putte, C. W. E. van Eijk, R. W. Hollander, H. U. Johner and C. Santoni, "Anti-proton Beam Monitoring System for CP LEAR," IEEE Trans. Nuc. Sci., NS-37, 53 (1990)

[2] 'R. Debbe, J. Fischer, D. Lissauer, T. Ludlam, D. Makowiecki, E. O'Brien, V. Radeka, S. Rescia, L. Rogers, G. C. Smith, D. Stephani, B. Yu, S. V. Greene, T. K. Hemmick, J. T. Mitchell, and B. Shivakumar, "A Study of Wire Chambers with Highly Segmented Cathode Pad Readout for High Multiplicity Charged Particle Detection," IEEE Trans. Nuc. Sci., NS-37, 88 (1990)

[3] G. Zanella and R. Zannoni, "X-Ray Imaging with Scintillating Glass Optical Fibres," Nucl. Instr. and Meth., A287, 619 (1990)

[4] T. Nakagawa and W. Bohne, "A Compact Gridless Channel Plate Start Detector," Nucl. Instr. and Meth., A271, 523 (1988)

[5] H. Roehrig, S. Nudelman, H. D. Fisher, M. M. Frost and M. P. Capp, "Photoelectronic Imaging for Radiology," IEEE Trans. Nuc. Sci., NS-28, 190 (1981)

[6] E. H. M. Heijne, L. Hubbeling, B. D. Hyams, P. Jarron, P. Lazeyras, F. Piuz, J. C. Vermeulen and A. Wylie, "A Silicon Surface Barrier Microstrip Detector Designed for High Energy Physics," Nucl. Instr. and Meth., 178, 331 (1980)

[7] E. Gatti, P. Rehak and J. T. Walton, "Silicon Drift Chambers - First Results and Optimum Processing of Signals," Nucl. Instr. and Meth., 226, 129 (1984)

[8] C. J. S. Damerell, F. J. M. Farley, A. R. Gillman and F. J. Wickens, "Charge Coupled Devices for Particle Detection with High Spatial Resolution," Nucl. Instr. and Meth., 185, 33 (1981)

[9] J. Kemmer, "Fabrication of Low Noise Silicon Radiation Detectors by the Planar Process," Nucl. Instr. and Meth., 169, 499 (1980)

[10] S. Holland and H. Spieler, "A Monolithically Integrated Detector-Preamplifier on High-Resistivity Silicon," IEEE Trans. Nuc. Sci., NS-37, 463 (1990)

[11] S. Gaalema, "Low Noise Random Access Readout Technique for Large Area PIN Detector Arrays, "IEEE Trans. Nuc. Sci., NS-32, 417 (1985)

[12] B. Diericky, J. Vermeiren and O. Frenzl, "The ISOPHOT CREA25 Far Infrared Si:P Matrix," Nucl. Instr. and Metn., A275, 527 (1989)

[13] G. F. Knoll, Radiation Detection and Measurement, John Wiley \& Sons, Inc., New York, p. 650 (1979)

[14] F. S. Goulding and D. A. Landis, "Signal Processing for Semiconductor Detectors," IEEE Trans. Nuc. Sci., NS-29. 1125 (1982) 
[15] V. Radeka, "Signal, Noise and Resolution in Position-Sensitive Detectors," IEEE Trans. Nuc. Sci., NS-21, 51 (1974)

[16] E. Beuville, K. Borer, E. Chesi, E. H. M. Heijne, P. Jarron, B. Liosowski, and S. Singh, "AMPLEX, A Low-Noise, Low-Power, Analog CMOS Signal Processor for Multielement Silicon Particle Detecotrs," Nucl. Instr. and Meth., A288, 157 (1990)

[17] S. A. Kleinfelder, W. C. Carithers, Jr., R. P. Ely, Jr., C. Harber, F. Kirsten, H. Spieler, "A Flexible 128 Channel Silicon Strip Detector Instrumentation Integrated Circuit with Sparse Data Readout," IEEE Trans. Nuc. Sci., NS-35, 171 (1988)

[18] J, S. Russ, R. J. Yarema, T. Zimmerman, "Studies of the LBL CMOS Integrated Amplifier/Discriminator for Randomly Timed Inputs from Fixed Target Experiments," IEEE Trans. Nuc. Sci., 36, 477 (1989)

[19] S. L. Thomas and P. Seller, "A Silicon Pixel Detectors with Routing for External VLSI Readout," Nucl. Instr. and Meth., A275, 564 (1989)

[20] B. Diericky, "XYW Detectors: A Smart Two-Dimensional Particle Sensor," Nucl. Instr. \& Meth., A275, 542 (1989)

[21] R. A. Street, S. Nelson, L. Antonuk and V. Perez-Mendez, "Amorphous Silicon Sensor Arrays for Radiating Imaging," Mat. Res. Soc. Symp. Proc., Vol. 192, 441 (1990)

[22] S. Parker, "A Proposed VLSI Pixel Device for Particle Detection," Nucl. Instr. and Meth., A275, 494 (1989)

[23] I. Shimizu, "Electro-photography," Semiconductors and Semimetals, Academic Press, Vol. 21, Part D, p. 55 (1984)

[24] S. Kaneko, "Solid-State Image Sensor," Semiconductors and Semimetals, Academic Press, Vol. 21, Part D, p. 139 (1984)

[25] S. N. Kaplan, J. Morel, V. Perez-Mendez and R. A. Street, "Detection of Charge Particles in Amorphous Silicon Layers," IEEE Trans. Nuc. Sci., NS-33, 351 (1986)

[26] W. Guang, H. Okamoto and Y. Hamakawa, "Amorphous-Silicon Photovoltaic XRay Sensor," Jap. J. Appl. Phys., Vol. 24, 1105 (1985)

[27] S. Qureshi, Hydrogenated Amorphous Silicon Radiation Detectors: Material Parameters; Radiation Hardness; Charge Collection, Ph.D. Thesis, UC Berkeley (1991)

[28] W. E. Spear and P. G. LeComber, "Substitutional Doping of Amorphous Silicon," Solid State Commun., Vol. 17, p. 1193 (1975)

[29] D. Adler, B. Schwartz, M. Steele, Physical Properties of Amorphous Materials, Plenum Press, p. 48 (1985) 
[30] M. H. Brodsky, M. A. Frisch, J. F. Ziegler and W. A. Lanford, "Quantitative Analysis of Hydrogen in Glow Discharge Amorphous Silicon," Appl. Phys. Lett., 30, 561 (1977)

[31] N. Shibata, K. Fukada, H. Otoshi, J. Hanna, S. Oda and I. Shimizu, "Preparation of Polycrystalline Silicon by Hydrogen-Radical-Enhanced Chemical Vapor Deposition," Jap. J. Appl. Phys., 26, L10 (1987)

[32] L. Ley, "Photoemission and Optical Properties," Chap. 3. in The Physics of Hydrogenated Amorphous Silicon II, edited by J. D. Joannopoulos and G. Lucovsky, Springer-Verlag, Berlin, p. 142 (1984)

[33] A. C. Hourd and W. E. Spear, "Determination of the Extended-state Electron Mobility in a-Si," Phil. Mag. B, Vol. 51, L13 (1985)

[34] D. G. Gouldie and W. E. Spear, "The Extended-state Hole Mobility in Amorphous Silicon," Phil. Mag. Lett., Vol. 57, 135 (1988)

[35] N. F. Mott, "Electrons in Disordered Structures," Adv. Phys., Vol. 16, p. 49 (1967)

[36] A. Madan and M. P. Shaw, The Physics and Applications of Amorphous Semiconductors, Academic Press, San Diego, p. 39 (1988)

[37] P. C. Tayler, "Magnetic Resonance Measurements in a-Si:H," Semiconductors and Semimetals, Academic Press, Vol. 21, Part C, p. 99 (1984)

[38] C. R. Wronski, "Staebler-Wronski Effect," Semiconductors and Semimetals, Academic Press, Vol. 21, Part C, p. 347 (1984)

[39] C. W. Bates, "Scintillation Process in Thin Films of CsI(Na) and CsI(Tl) due to Low Energy X-rays, Electrons and Protons," Adv. Electronics and Electron. Phys., 28A, 451 (1968)

[40] Pyralin polyimide coating P1-2555, Dupont Electronics, Wilmingston, Delaware.

[41] I. Fujieda, G. Cho, J. Drewery, T. Gee, T. Jing, S. N. Kaplan, V. Perez-Mendez, D. Wildermuth and R.A. Street, "X-ray and Charged Particle Detection with CsI(Tl) Layer Coupled to a-Si:H Photodiode Layers," IEEE Trans. Nuc. Sci., NS38,255 (1991)

[42] A. L. N. Stevels and A. D. M. Schrama de Pauw, "Vapor-Deposited CsI:Na Layers, I. Morphologic and Crystallographic Properties, II. Screens for Application in X-ray Imaging Devices," Philips Res. Repts., 29, 340 (1974)

[43] H. Ito, S. Matsubara, T. Takahashi, T. Shimada and H. Takeuchi, "Integrated Radiation Detectors with a-Si Photodiodes on Ceramic Scintillators," Jap. J. Appl. Phys., Vol., 28, L1476 (1989)

[44] I. Holl, E. Lorenz and G. Mageras, "A Measurement of Light Yield of Some Common Inorganic Scintillators," IEEE. Trans. Nuc. Sci., NS-35, 105 (1989) 
[45] V. Perez-Mendez, S. N. Kaplan, G. Cho, I. Fujieda, S. Qureshi, W. Ward and R. A. Street, "Hydrogenated Amorphous Silicon Pixel Detectors for Minimum Ionizing Particles," Nucl. Instr. and Meth., A273, 127 (1988)

[46] M. Kobayashi and S. Sakuragi, "Radiation Damage of CsI(Tl) Crystal above $10^{3}$ rad," Nucl. Instr. and Meth., A254, 275 (1987)

[47] C. L. Woody, P. W. Levy, J. A. Kierstead, T. Skwarnicki, Z. Sobolewski, M. Goldberg, N. Horwitz, P. Souder, D. F. Anderson, "Readout Techniques and Radiation Damage of Undoped CsI," IEEE Trans. Nuc. Sci., NS-37, 497 (1990)

[48] H. Curtins, W. Wyrsch and A. V. Shah, "High-Rate Deposition of a-Si:H Films by VHF Plasma CVD," Electron. Lett., 23, 228 (1987) 


\section{Chapter 3 Charge Collection and Signal Formation}

\subsection{Introduction}

When ionizing radiations, such as $\mathrm{X}$-rays, gamma-rays, or charged particles, are incident on an amorphous silicon pin diode they produce electron-hole pairs along their paths. These electrons and holes drift toward the $\mathrm{n}$ - and p-contact layers under the strong electric field built by the reverse bias. The drift motion of electrons and holes induces a current pulse at the external circuit of the detector with a shape determined by the electric properties and the geometric configuration of the detector. The main interaction and drift occurs in thick i-layer of the diode. The thin $(40 \sim 100 \mathrm{~nm}) \mathrm{p}$ - and n-layers serve as blocking contacts to minimize the unwanted injection of electrons and holes from metal contacts when the high reverse bias is applied. The signal current pulse is integrated by a charge sensitive preamplifier and is shaped by a following shaping amplifier to produce a voltage pulse whose magnitude is proportional to the signal charge.

Normalization of the output signal charge to the total generated charge is called the collection efficiency. This is an important quantity for optimal design of the detector, for example, to determine of the size and thickness of pixel detectors, the operation bias, the shaping time, etc.

In this chapter simple analytical equations of the charge collection efficiency will be derived and measurements of the required device parameters and simulation of the model in several cases will be discussed. 


\subsection{Charge Collection Efficiency}

The charge collection efficiency, $\eta$, is defined as

$$
\eta \equiv \frac{\text { collected charge }}{\text { total generated charge }}=\frac{Q_{\text {col }}}{Q_{\text {gen }}}
$$

The total generated charge, $Q_{\text {gen }}$, is

$$
Q_{\text {gen }}=\int_{0}^{d} q \frac{\Delta E(x)}{W} d x=q \int_{0}^{d} n_{0}(x) d x=q N_{0}
$$

where $\mathrm{q}$ is an electronic charge and $\mathrm{N}$ is the total number of generated electron-hole pairs. $\mathrm{d}$ is the thickness of the i-layer of a-Si:H pin diodes. $\Delta \mathrm{E}(\mathrm{x})$ is the energy deposited in the detector by the radiation at a distance $x$ measured from $p-i$ junction. $W$ is the average ionization energy needed to produce an electron-hole pair by the radiation and its value varies slightly depending on the radiation type and its kinetic energy, $n_{0}(x)$ is the initial distribution of generated charges and $\mathrm{N}_{0}$ is the total number of electron-hole pairs produced.

The collected signal charge can be obtained by integration of the induced current caused by the motion of signal charges in the detsting material.

$$
\mathrm{Q}_{\text {col }}=\int_{0}^{\mathrm{T}_{\mathrm{i}}} \mathrm{I}_{\text {ind }}(\mathrm{t}) \mathrm{dt}=\mathrm{q} \mathrm{N}_{\text {col }}
$$

where $T_{i}$ is the integration time which is the less between the transit time of charges in the detector and the shaping time of the pulse shaping amplifier. In gas-type radiation detectors, such as ionization chambers, the induced signal current is normally calculated by the energy balance, but in solid state detectors, such as p-n diodes or Schottky diodes, it should be calculated according to principles developed in the study of induced currents in vacuum tubes.[1] Tove et al [2] have showed that a moving point charge $q$ between two parallel electrode with separation $d$, induces a current, $i$ which is

$$
\mathrm{i}=\left(\frac{\mathrm{q}}{\mathrm{d}}\right) \times \frac{\mathrm{dx}}{\mathrm{dt}}
$$


Several assumptions are made in order to calculate the signal charge collection in a highly reverse biased a-Si:H pin detector ;

(1) Charge generation : Two cases of distribution of generated charge density are considered as shown in Fig. 3.1; (a) Uniform generation : $n_{0}(x)=$ constant $=n_{0}$. Radiations, such as minimum ionizing particles which have a much longer range than the typical thickness of the i-layer $(5 \sim 50 \mu \mathrm{m})$, produce a uniform distribution of charge density. In partial depletion cases, the charges produced in the depletion region contribute to the total signal size. (b) Surface generation : Radiation such as ultra violet light has a large absorption coefficient so effectively all the interaction occurs at the surface region of the detector. Generally the density distribution is expressed as an exponential function,

$$
n_{0}(x)=n_{0} e^{-a x}
$$

where $a$ is the linear absorption coefficient and it is the reciprocal of the mean free path of the radiation in the a-Si:H.

In the case of the strong absorption where the mean free path is very short compared to the diode thickness ( $a d \gg 1$ ), the density distribution can be modelled simply as a delta function, $n_{0}(x)=N_{0} \delta(x)$, and only one type of charge contributes to the signal.
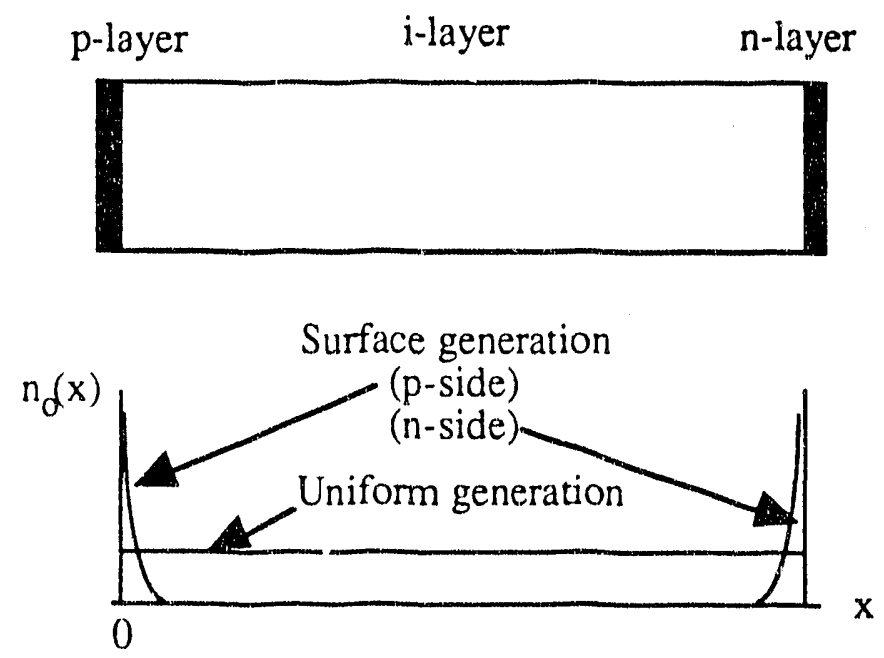

Fig. 3.1 A schematic geometry of an a-Si:H pin detector and the density distribution of generated charges; (a) Surface generation at $\mathrm{p}$-side and $\mathrm{n}$-side, (b) Uniform generation. 
(2) Diffusion and drift: The diffusion of charge carriers during the transit time is neglected because the diffusion length is much shorter than the sample thickness. Therefore the drift is the only driving force of the motion of electrons and holes considered in the calculation. Also the mobilities of electrons and holes are assumed to be constant and independent of the electric field strength. These assumptions are not good especially in the case of hole transport because of their low mobility and hopping characteristics. However, the simulation seems to give good fits to the measurements. For simplicity of the calculation we neglect the dispersive nature of holes.

(3) Recombination and trapping : The main recombination process is Shockly-Read-Hall recombination through the donor-like states located in the middle of the gap which is mainly the $\mathrm{D}^{\circ}$ states of the silicon dangling bonds.[3] In good quality amorphous silicon, other gap states, for example inpurities like $\mathrm{P}, \mathrm{B}, \mathrm{O}, \mathrm{C}$, and $\mathrm{N}$ etc. or hydrogen-complex induced trap centers, are also important recombination and deep trapping centers causing the signal loss during the transit time.[4] The direct recombination (Auger recombination) of generated electrons and holes is neglected because of the small cross section. The overall charged carrier loss process is modelled as a simple exponential function during the transit time using the concept of an average lifetime, $\tau_{\mathrm{i}}$, where the subscript $i$ refers to electrons $\left(\tau_{e}\right)$ or holes $\left(\tau_{h}\right)$. The density of charge carriers at a time $\mathfrak{t}$ measured from the generation time is

$$
n(t)=n(0) e^{-t / \tau_{i}}
$$

This assumption is generally valid in the case of medical $x$-ray imaging and detection of minimum ionizing particles, because the density of generated signal charge is so small compared to the large density of recombination centers that charge loss is independent of position. In the case of highly charged heavy particle detection such as alpha particles, protons and fission fragments, this assumption is poor and a plasma effect, leading to slow pulse rise time due to plasma formation [5] and a pulse height defect due to high rate of the direct recombination [6] of electrons and holes, must be considered. 
(4) Electric field configuration: The abrupt depletion model is used to calculate the electric field in the pin diode. The electric field in the $\mathrm{p}$ - and n-layer are neglected. In the i-layer the dangling bonds which do not have hydrogen atoms attached are readily ionized under the action of a reverse bias across the diode, and will leave a density of positive space charge which is $30 \sim 35 \%$ of the density of the total dangling bonds as measured by electron spin resonance.[7] Therefore the i-layer behaves as a slightly n-type layer and the depletion of the $i$ layer starts from the $p-i$ junction and extends towards the $i-n$ junction as the reverse bias increases. For maximum signal charge collection, the diodes must be fully depleted.

When the applied bias is high enough to deplete all the i-layer the electric field is simply linear, however when the bias is not high enough to deplete all the i-layer, the electric field in the $\mathrm{i}$-layer of reverse biased a-Si:H pin diode is normally calculated by a two region approximation based on the electron quasi Fermi energy level. Recently Kleider et al.[8] have developed a model to calculate the electric field in amorphous silicon Schottky diodes which is based on both electron and hole quasi Fermi energy levels and results in a three-region approximation. However for the calculation of the charge collection in reverse biased thick pin diodes the two-region approximation seems to be satisfactory. because the other region width is normally less than $1 \mu \mathrm{m}$.

In region I, where the electric potential is higher than a critical voltage $\Phi_{\mathrm{c}}(\sim-1 \mathrm{~V})$, the space charge density is constant and equal $\mathrm{qN}_{\mathrm{d}}{ }^{*}$ where $\mathrm{N}_{\mathrm{d}}{ }^{*}$ is the maximum ionizable dangling bond density. The Poisson equation has the form

$$
\frac{d^{2} \Phi(x)}{d x^{2}}=-\rho \equiv \frac{-q N_{d}^{*}}{\varepsilon_{0} \varepsilon_{a s i}}
$$

where $\varepsilon_{\mathrm{o}}$ is the dielectric constant of vacuum and $\varepsilon_{\mathrm{asi}}$ is the relative dielectric constant of hydrogenated amorphous silicon.

In region II where the potential $\Phi(\mathrm{x})$ drops below $\Phi_{\mathfrak{c}}$, we assume the space charge is proportional to the potential $\Phi(x)$, therefore the Poisson equation is

$$
\frac{\mathrm{d}^{2} \Phi(\mathrm{x})}{\mathrm{dx^{2 }}}=-\rho \times \frac{\Phi(\mathrm{x})}{\Phi_{\mathrm{c}}}
$$


The equations are then solved with the i-layer boundary condition of $\Phi(0)=V_{a}=$ the applied reverse bias, and $\Phi(\mathrm{d})=0$ and the continuity condition of potential and field at the boundary between two regions.

However the transport of charge in region II does not contribute to the signal charge collection because the field is so low that deep trapping and recombination are dominant in this region. Therefore we will neglect region II and only consider region I. See Fig. 3.2 for comparison of electric fields and space charge density distribution in the case of a tworegion approximation and a one-region approximation.

The thickness of region I, which is called the depletion thickness, $w$, is $w=\sqrt{\frac{2 V_{a}}{\rho}}$

In order to deplete all of the $\mathrm{i}$-layer, the applied reverse bias, $\mathrm{V}_{\mathrm{a}}$, must exceed the full depletion bias, $V_{f}$, which is obtained from the above equation by substituting $w$ by $d$.

$$
V_{\mathrm{f}}=\frac{\rho \mathrm{d}^{2}}{2}
$$
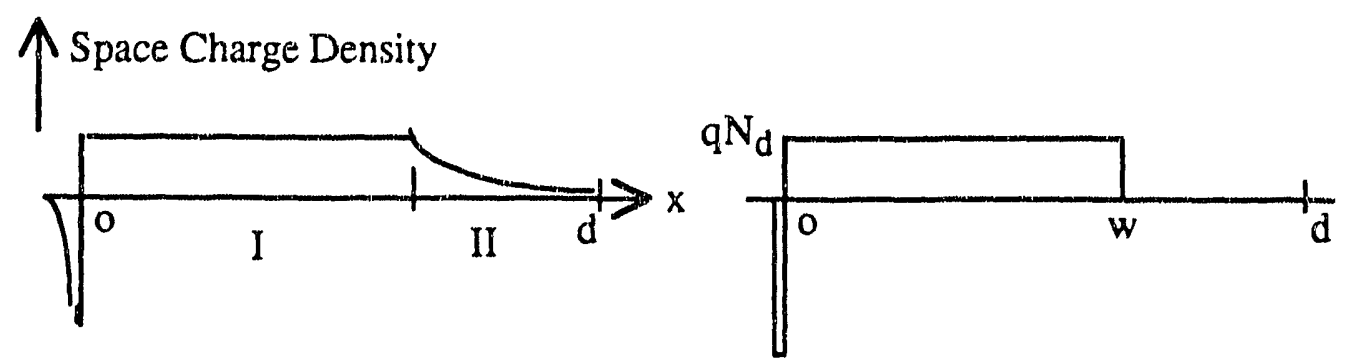

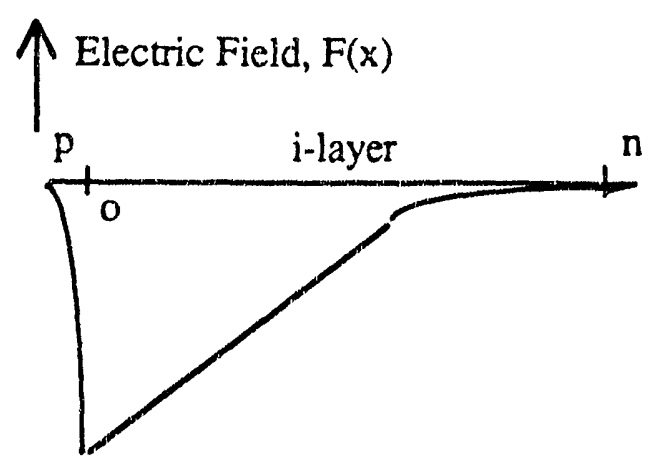

(a)

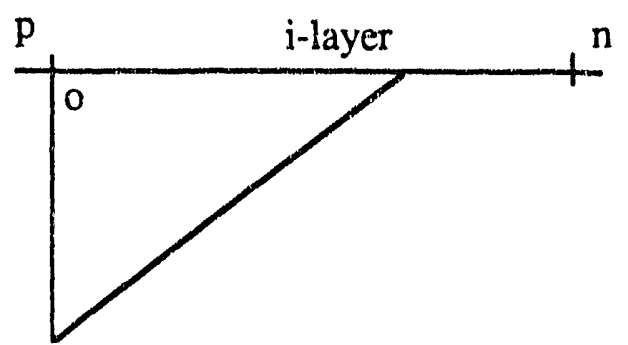

(b)

Fig. 3.2 A schernatic diagram of space charge and electric field distribution in a partially depleted a-Si:H pin diode depicted by (a) two-region model and (b) one-region model. 
Now let's define a dimensionless variable, the depletion parameter, $Y$.

$$
\mathrm{Y} \equiv \frac{\mathrm{w}}{\mathrm{d}}=\left(\frac{\mathrm{V}_{\mathrm{a}}}{\mathrm{V}_{\mathrm{f}}}\right)^{1 / 2}
$$

Then the electric field $F(x)$ in the partial depletion case is

$$
\begin{array}{ll}
F(x)=\rho(x-w) & 0 \leq x \leq w \quad(Y \leq 1 ; \text { partial depletion }) \\
F(x)=0 & w \leq x \leq d
\end{array}
$$

In the full depletion case,

$$
F(x)=\rho\left(x-\frac{d}{2}-\frac{w^{2}}{2 d}\right) \quad 0 \leq x \leq d \quad(Y \geq 1 ; \text { full depletion })
$$

where $w$ does not have a physical meaning of the depletion thickness because it is larger than the i-layer thickness $\mathrm{d}$ from its definition.

(5) Ballistic Deficit: The output pulse height of the shaping amplifier does not reflect the actual charge collection efficiency when the pulse shaping time, $\tau$, is shorter than the transit time, $\mathfrak{t}_{\mathfrak{t}}$, of the charge carriers in the detector. The output signal is only proportional to the charges collected during a time interval equal to the shaping time, i.e. $t_{t}$ must be replaced by $\tau$ in the integration to calculate $Q_{\text {col }}$. This type of signal loss is called the ballistic deficit.[9] In principle the ballistic deficit is not a charge loss process in the detector, but it affects the output signal size as do other charge loss mechanisms. For thick a-Si:H pin detectors $(>10$ $\mu \mathrm{m})$, the ballistic deficit is a major signal loss in the hole collection because of its low drift mobility and long transit time especially when a fast pulse shaping ( $\tau<1 \mu \mathrm{sec})$ is used. In the electron collection, Ballistic deficit is negligible unless the sample diode is very thick (> $100 \mu \mathrm{m})$ and the operation bias is low.

Using those assumptions, the charge collection efficiency is obtained in simple analytical forms for three cases; uniform generation and the two surface generation cases in a simple pin detector and also analyzed was uniform generation case in a multi buried layer detector. 


\subsubsection{Case 1 : Uniform Generation in PIN Detector}

The charge collection efficiency is calculated in the case of uniform distribution of charge density, $n_{0}(x)=n_{0}$, for two bias conditions; partial depletion and full depletion bias.

(1) Partial depletion bias $(\mathrm{Y}<1$ ):

(a) Electron collection efficiency

In a partial depletion case, the generated electrons drift from the $\mathrm{p}$ contact toward the depletion boundary, w. The drift velocity of the charge carrier in the semiconductor is proportional to the electric field, to a first order approximation, so the velocity of an electron at position $\mathrm{x}$ will be given by the following equation, using the electric field distribution for the partial depletion case.

$$
v_{e}(x)=-\mu_{e} F(x)=\frac{w-x}{t_{n}}
$$

where $\tau_{n}$ is the electron characteristic time defined by

$$
\tau_{\mathrm{n}} \equiv \frac{1}{\rho \mu_{\mathrm{e}}}
$$

where $p$ is defined in the previous section.

By the definition of velocity,

$$
v(x) \equiv \frac{d x}{d t}
$$

the electron drift velocity can be represented as a function of time, $t$, and the initial position, $x_{0}$, instead of the present position, $x$.

$$
v_{e}\left(x_{0}, t\right)=\frac{\left(w-x_{0}\right)}{\tau_{n}} e^{-\frac{1}{\tau_{n}}}
$$

The transit time of the electron from $x_{0}$ to $w$ is infinite. Theoretically the electron never reaches the position $w$. This applies to any electron between $x_{0}=0$ and $x_{0}=w$. In the case of uniform generation of charge carriers, the induced current, $\Delta \mathrm{I}_{\mathrm{e}}$ due to $\mathrm{n}_{\mathrm{o}}$ electrons generated at $x=x_{0}$ is given by Ramo's theorem.

$$
\Delta \mathrm{I}_{\mathrm{e}}\left(\mathrm{x}_{0}, \mathrm{t}\right)=q \mathrm{n}_{\mathrm{o}} \mathrm{e}^{-\frac{1}{\tau_{\mathrm{e}}}} \frac{\mathrm{v}_{\mathrm{e}}\left(\mathrm{x}_{0}, \mathrm{t}\right)}{\mathrm{d}}
$$


where $\tau_{e}$ is the electron lifetime and the exponential term is a correction factor due to the the loss of electrons by trapping which is mostly a deep trapping by dangling bonds. Now, consider the charge collection using an ideal charge sensitive preamplifier which is simply a current integrator having a collection or integration time $\tau$. The induced charge at the electrode due to the $n$ electrons generated at $x_{0}$, is

$$
\Delta \mathrm{Q}_{\mathrm{e}}\left(\mathrm{x}_{0}\right)=\int_{0}^{\tau} \Delta \mathrm{I}_{\mathrm{e}}\left(\mathrm{x}_{0}, \mathrm{t}\right) \mathrm{dt}
$$

The total induced charge due to electrons generated in the depletion region $\left(0 \leq x_{0} \leq w\right)$ is

$$
\mathrm{Q}_{\mathrm{e}}=\int_{0}^{\mathrm{w}} \Delta \mathrm{Q}_{\mathrm{e}}\left(\mathrm{x}_{\mathrm{o}}\right) \mathrm{d} \mathrm{x}_{\mathrm{o}}=\int_{0}^{\mathrm{w}} \mathrm{dx} \mathrm{x}_{\mathrm{o}} \int_{0}^{\tau} \Delta \mathrm{I}_{\mathrm{e}}\left(\mathrm{x}_{0}, \mathrm{t}\right) \mathrm{dt}
$$

Since there is no field in the undepleted region, there is no contribution to the signal from carriers in this region. The maximum induced charge at the electrode by collecting all the electrons and holes in the detector is

$$
\mathrm{Q}_{\text {gen }}=\mathrm{q} \mathrm{n}_{\mathrm{o}} \mathrm{d}
$$

So the electron collection efficiency, the relative signal size contributed by the electrons is

$$
\eta_{\mathrm{e}} \equiv \frac{\mathrm{Q}_{\mathrm{e}}}{\mathrm{Q}_{\mathrm{gen}}}=\frac{\mathrm{Y}^{2}}{2} \times \frac{1-\mathrm{e}^{-\alpha_{\mathrm{e}} \tau}}{\alpha_{\mathrm{c}} \mathrm{n}}
$$

where

$$
\alpha_{e} \equiv \frac{\tau_{n}+\tau_{e}}{\tau_{n} \tau_{e}}
$$

If we collect all the electrons from the detector $\eta_{\mathrm{e}}$ is equal to 0.5 .

(b) Hole collection efficiency

Similarly, the drift velocity ror holes is

$$
v_{h}(x)=\mu_{h} F(x)=\frac{x-w}{\tau_{p}}
$$

where $\tau_{p}$ is the hole characteristic time defined by

$$
\tau_{\mathrm{p}} \equiv \frac{1}{\rho \mu_{\mathrm{h}}}
$$

Using the same process as for the electrons, the velocity of holes at $\mathrm{x}$ is represented by the initial position $\mathrm{x}_{0}$, and time $\mathrm{t}$. 


$$
v_{h}\left(x_{0}, t\right)=\frac{x_{0}-w}{\tau_{p}} e^{\frac{t}{\tau_{p}}}
$$

The difference between the collection process of holes and of electrons is that the electrons can never reach the depletion boundary, $w$, for any given collection time, $t_{s}$ but some of holes can arrive at the p-contact. Therefore the above velocity expression is only true under the condition that

$$
t<t_{p} \equiv \tau_{p} \ln \left(\frac{w}{w-x_{0}}\right)
$$

where $t_{p}$ is the transit time of the holes from $x=x_{0}$ to $x=0$. The current density is also limited up to $t_{p}$.

$$
\Delta I_{h}\left(x_{0}, t\right)=-q n_{0} e^{-\frac{1}{\tau_{h}}} \frac{v_{h}\left(x_{0}, t\right)}{d} H\left(t_{p}-t\right)
$$

where $t_{h}$ is the hole lifetime and $H(t)$ is the step function. If the collection time, $\tau$ is specified, then a division should be made between two groups of holes; one group consists of the holes which are collected completely during that time and the other consists of the holes which remain in the i-layer at time $\tau$. The boundary between the initial positions of the two groups of holes is

$$
\mathrm{x}_{\mathrm{p}}=\mathrm{w}\left(1-\mathrm{e}^{-\frac{\tau}{\tau_{\mathrm{p}}}}\right)
$$

The induced charge per unit area of the electrode by collection of holes is

$$
Q_{h}=\int_{0}^{x_{p}} d x_{0} \int_{0}^{t_{p}} \Delta I_{h}\left(x_{0}, t\right) d t+\int_{x_{p}}^{w} d x_{0} \int_{0}^{\tau} \Delta I_{h}\left(x_{0}, t\right) d t
$$

So the collection efficiency contributed by holes is

$$
\eta_{h} \equiv \frac{Q_{h}}{Q_{g e n}}=\frac{Y^{2}}{2} \times \frac{1-e^{-\alpha_{h} \tau}}{\alpha_{h} \tau_{p}}
$$

where

$$
\alpha_{h} \equiv \frac{\tau_{p}+\tau_{h}}{\tau_{p} \tau_{h}}
$$

(c) Total collection efficiency

Finally the total collection efficiency for both electrons and holes in a partially depleted detector for a given shaping time $\tau$, is

$$
\eta=\eta_{e}+\eta_{h}=\frac{Y^{2}}{2} \times\left[\frac{1-e^{-\alpha_{e} \tau}}{\alpha_{e} \tau_{n}}+\frac{1-e^{-\alpha_{h} \tau}}{\alpha_{h} \tau_{p}}\right]
$$




\section{(2) Eull Depletion Bias ( $Y>1$ ):}

(a) Electron collection efficiency

The procedure for calculating the collection efficiency for the full depletion case is the same as for the partial depletion case except that we need to compare the collection time, $\tau$, and the maximum transit time, $t_{n}$, which is the electron travel time from $x=0$ to $x=d$ given by

$$
t_{n} \equiv \tau_{n} \ln \left(\frac{Y^{2}+1}{Y^{2}-1}\right)
$$

The calculated electron collection efficiency is

$$
\eta_{\mathrm{e}}=\frac{1}{8}\left[\left(\mathrm{Y}^{2}+1\right)^{2} \frac{1-\mathrm{e}^{-\alpha_{\mathrm{e}} t}}{\alpha_{\mathrm{e}} \tau_{\mathrm{n}}}-\left(\mathrm{Y}^{2}-1\right)^{2} \frac{1-\mathrm{e}^{-\beta_{\mathrm{e}} \mathrm{t}}}{\beta_{\mathrm{e}} \tau_{\mathrm{n}}}\right]
$$

where

$$
\beta_{e} \equiv \frac{\tau_{n}-\tau_{e}}{\tau_{n} \tau_{e}}
$$

and $t$ is the lesser of the two $\tau$ and $t_{n}$. This time governs the collection process.

(b) Hole collection efficiency

Similarly the hole collection efficiency is

$$
\eta_{h}=\frac{1}{8}\left[\left(Y^{2}+1\right)^{2} \frac{1-e^{-\alpha_{h} t}}{\alpha_{h} \tau_{p}}-\left(Y^{2}-1\right)^{2} \frac{1-e^{-\beta_{h} t}}{\beta_{h} \tau_{p}}\right]
$$

where

$$
\beta_{h} \equiv \frac{\tau_{p}-\tau_{h}}{\tau_{p} \tau_{h}}
$$

and $t$ is the minimum between the shaping time $\tau$ and the hole transit time $t_{p}$,

$$
t_{p} \equiv \tau_{p} \ln \left(\frac{Y^{2}+1}{Y^{2}-1}\right)
$$

(c) Total collection efficiency

The total collection efficiency is

$$
\eta=\eta_{\mathrm{e}}+\eta_{\mathrm{h}}
$$




\subsubsection{Case 2 : P-side Surface Generation in PIN Detector}

When strongly absorbed radiation is incident on the $\mathrm{p}$-side of a-Si:H pin detectors, the signal is induced by the electrons only, because the transit distance of the holes are nearly zero. Hence

$$
\eta=\frac{Q_{e}+Q_{h}}{Q_{g e n}} \cong \frac{Q_{e}}{Q_{g e n}}
$$

where

$$
Q_{\text {gen }}=q N_{0}
$$

By Ramo's theorem, the induced current is

$$
I_{e}(t)=q N_{0} e^{-\frac{t}{\tau_{e}}} \frac{v_{e}(t)}{d}
$$

and the collected electron charge is

$$
\mathrm{Q}_{e}=\int_{0}^{\mathrm{t}} \mathrm{I}_{\mathrm{e}}(\mathrm{t}) \mathrm{dt}
$$

Hence the collection efficiency is

$$
\eta=\frac{Q_{e}}{Q_{g e n}}=\frac{1}{d} \times \int_{0}^{t} e^{-\frac{1}{\tau_{e}}} v_{e}(t) d t
$$

where $t$ is the lesser of two times: the transit tirne and the shaping time.

(1) Partiai depletion bias $(Y<1)$ :

In the case of partial depletion, the electrons never arrive at the n-side during the shaping time and their velocity can be represented by

$$
v_{e}(t)=\frac{w}{\tau_{n}} e^{-\frac{1}{\tau_{n}}}
$$

Therefore, the collection efficiency is

$$
\eta=Y \times \frac{1-e^{-} \alpha_{e} \tau}{\alpha_{e} \tau_{n}}
$$

where $\tau_{n}$ and $\alpha_{e}$ are defined already in equations (3.5) and (3.6) respectively.

(2) Full Depletion Bias $(Y>1)$ :

The electron drift velocity in the full depletion case is 


$$
v_{e}(t)=\frac{\frac{d}{2}-\frac{w^{2}}{2 d}}{\tau_{n}} e^{-\frac{t}{\tau_{n}}}
$$

If the shaping time $\tau<t_{n}$, which is the transit time from $x=0$ to $x=d$, then the collection is terminated at time $t=\tau$ and $\tau>t_{n}$ the collection is terminated at time $t=t_{n}$. Hence the collection efficiency is

$$
\eta=\left(\frac{Y^{2}+1}{2}\right) \times \frac{1-e^{-\alpha_{e} t}}{\alpha_{e} \tau_{n}}
$$

where $t$ is the less of two, $\tau$ and $t_{n}$.

\subsubsection{Case 3 : N-side Surface Generation in PIN Detector}

In this case, the signal is governed by the hole collection only.

(1) Partial depletion bias $(Y<1)$ :

When the $\mathrm{i}-\mathrm{layer}$ is not fully depleted, there is no external field at $\mathrm{n}-\mathrm{i}$ junction, so the holes can not drift. Therefore the collection efficiency is zero.

(2) Full Depletion Bias $(Y>1)$ :

Similarly to the electron case, the collection efficiency is

$$
\eta \cong \frac{\mathrm{Q}_{\mathrm{h}}}{\mathrm{Q}_{\mathrm{gen}}}=\frac{1}{\mathrm{~d}} \times \int_{0}^{\mathrm{t}} \mathrm{e}^{-\frac{\mathrm{t}}{\tau_{\mathrm{h}}}} \mathrm{v}_{\mathrm{h}}(\mathrm{t}) \mathrm{dt}
$$

where the hole drift velocity is

$$
v_{h}(t)=\frac{\frac{d}{2}-\frac{w^{2}}{2 d}}{\tau_{p}} \frac{t}{e \tau_{p}}
$$

Hence the collection efficiency is

$$
\eta=\left(\frac{Y^{2}-1}{2}\right) \times \frac{1-e^{-} \beta_{h} t}{\beta_{h} \tau_{p}}
$$

where $t$ is the lesser of two, the shaping time $\tau$ and the hole transit time $t_{p}$. Since the hole mobility is so low the collection is usually limited by the shaping time. 


\subsection{Signal Formation}

The radiation detector signal is usually processed as a voltage pulse in the following electronic system and the information is represented as a pulse height.

Fig. 3.3 shows a typical radiation detection system with ac coupling of the detector to a charge sensitive preamplifier.[10] First the signal current induced by the motion of the generated charges is integrated in a charge sensitive preamplifier. The output voltage signal of the preamplifier is shaped into a pulse form by the following shaping amplifier.

There are many types of pulse shaping amplifiers [11] depending on the specific purpose but here the discussion is limited only to the case of the most popular CR-(RC) ${ }^{\mathrm{n}}$ shaping amplifier which is basically composed of one differential (CR) stage and n stages of integration (RC). This produces a pseudo gaussian pulse when $\mathrm{n}$ is large. A typical value of $n$ is 4.[12]

\subsubsection{Output Signal of Charge Sensitive Preamplifier}

The total induced current from the detector can be written by

$$
I_{\text {ind }}(t)=Q_{\text {gen }} \times \frac{d \eta(t)}{d t}
$$

where $\eta(t)$ is the charge collection efficiency at a time $t$.

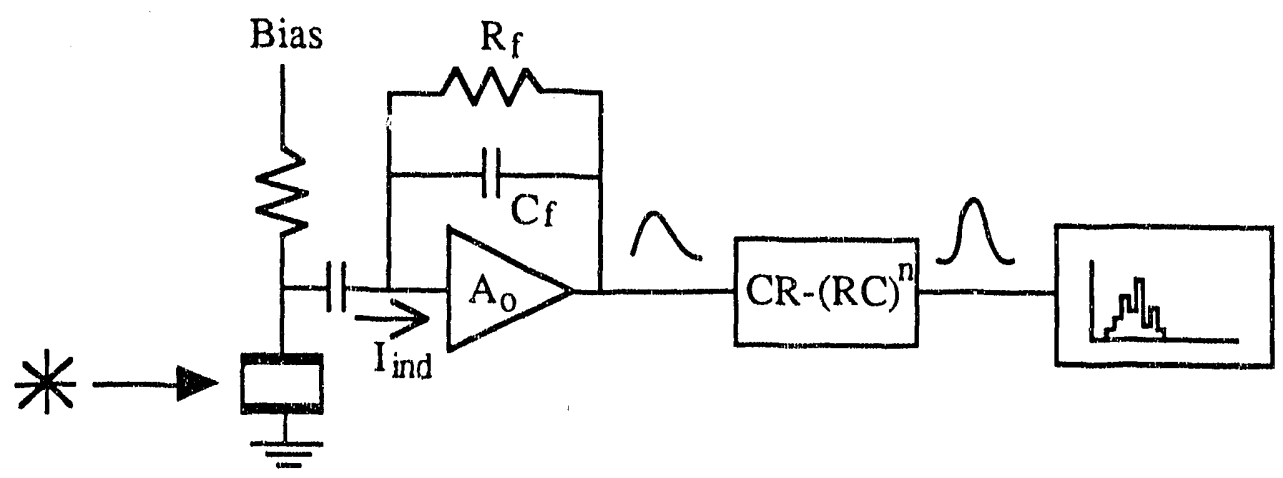

Radiation Detector Preamplifier Shaping Amplifier Pulse Analyzer

Fig. 3.3 A schematic diagram of radiation detection system. 
The input voltage signal to the preamplifier without the feedback loops is

$$
V_{i}(t)=\frac{1}{C_{t o t}} \int_{0}^{t} I_{\text {ind }}(t) d t^{\prime}
$$

where $C_{\text {tot }}$ is the sum of the detector capacitance, $C_{d}$, and the input capacitance of the charge-sensitive amplifier, $\mathrm{C}_{\mathrm{i}}$.

The closed-loop gain of the charge sensitive preamplifier, $A_{c}$, with a negative capacitive feedback [13] is calculated to be

$$
A_{c}=\frac{C_{t o t} \times A_{0}}{\left[C_{t o r}+\left(1+A_{o}\right) \times C_{f}\right]}
$$

If the open-loop gain, $A_{0}$, is large then $A_{c} \approx C_{\text {tot }} / C_{f}$.

If we consider the output pulse decay through the feedback resistor $R_{f}$ with a decay time constant, $\tau_{\mathrm{f}}=\mathrm{R}_{\mathrm{f}} \times \mathrm{C}_{\mathrm{f}}$, then the output signal becomes simply

$$
V_{c s a}(t) \approx \frac{1}{C_{f}} \times e^{-t / \tau_{f}} \int_{0}^{t} I_{\text {ind }}\left(t^{\prime}\right) \times e^{i / \tau_{f}} d t^{\prime}
$$

Now the output pulse height from the charge-sensitive preamplifier without pulse decay becomes simply

$$
V_{c s a}(t)=\frac{1}{C_{f}} \int_{0}^{t} I_{\text {ind }}\left(t^{\prime}\right) d t^{\prime}=\frac{Q_{g e n}}{C_{f}} \times \eta(t)
$$

i.e., the output pulse height from an ideal charge-sensitive preamplifier is simply proportional to the charge collection efficiency.

\subsubsection{Output Signal of CR-(RC) $)^{\mathrm{n}}$ Shaping Amplifier}

The output pulse shape of the $\mathrm{CR}-(\mathrm{RC})^{\mathrm{n}}$ shaping amplifier is calculated by a differentiating and $n$ integrating the output signal of the charge sensitive preamplifier.

$$
V_{\text {sha }}(t)=\int_{0}^{t} d t * * * \int_{0}^{t} d t \int_{0}^{t} d t\left(\frac{d V_{c s a}(t)}{d t}\right)
$$


Appendix $\mathrm{A}$ is a fortran program to calculate the charge collection efficiency and the normalized output signal of the shaping amplifier.

\subsection{Input Parameters}

In order to estimate the charge collection efficiency of a-Si:H pin detectors, several material parameters are required besides the operational parameters, such as bias and shaping time. They are the average ionization energy, the ionized dangling bond density, mobilities and lifetimes of electrons and holes in i-layer of a-Si:H. In this section, measurement and the results of these material-dependent parameters will be explained.

Samples used in this research were made by the PECVD method in three places: (a) thick $(10 \sim 50 \mu \mathrm{m})$ a-Si:H pin diodes from Glasstech Solar Inc. (GSI), now Material Research Group (MRG), (b) thin $(<1 \mu \mathrm{m})$ pin diodes and a-Si:H and poly-Si TFTs from Xerox Palo Alto Research Center (Xerox PARC) and (c) thin $(1 \sim 5 \mu \mathrm{m})$ pin diodes and buried layer diodes from Lawrence Berkeley Laboratory (LBL).

\subsubsection{Average Ionization Energy}

The average ionization energy, $\mathrm{W}$, of hydrogenated amorphous silicon was measured at LBL [14] using a $5 \mu \mathrm{m}$ thick a-Si:H detector and a pulsed X-ray source calibrated with $\sim 200 \mu \mathrm{m}$ thick crystalline silicon detector. For minimum ionizing particles it vas also measured at the Bevatron by Shafi.[15] The measured value was $4.8 \pm 0.2 \mathrm{eV}$. This is consistent with an empirical relationship between $\mathrm{W}$ and the band-gap energy $\mathrm{E}_{\mathrm{g}}$ reported by Klein.[16]

$$
\mathrm{W}=2.8 \times \mathrm{E}_{\mathrm{g}}+\text { phonon loss }=2.8 \times 1.7+0.5=5.2(\mathrm{eV})
$$




\subsubsection{Ionized Dangling Bond Density}

Good quality hydrogenated amorphous silicon normally has a dangling bond density of $10^{15} \sim 10^{16}$ per $\mathrm{cm}^{3}$. Dangling bonds are ionized by releasing the non-bonded electrons under the electric field and thus becoming positively charged. The maximum ionizable dangling bond density, $\mathrm{N}_{d}{ }^{*}$, under strong reverse bias was measured to be about one third of the total dangling bonds as measured by the electron spin resonance technique.[17] Two methods were used to measure the ionized dangling bond density directly :

(1) Hole onset : For charge generated at the $n-i$ interface by a short-wave-length light (500 $\sim 600 \mu \mathrm{m}$ ), there will be no hole collection signal until the i-layer is fully depleted. Hence the ionized dangling bond density can be calculated by the following equation converted from the equations (3.1) and (3.2) knowing the thickness of the sample and the threshold bias for full depletion, $\mathrm{V}_{\mathrm{f}}$,

$$
\mathrm{N}_{\mathrm{d}}^{*}=\frac{2 \varepsilon_{0} \varepsilon_{\mathrm{asi}} V_{\mathrm{f}}}{\mathrm{qd}^{2}}
$$

(2) Transient photoconductivity (DC time-of-flight): This method has been used to study the built-in potential distribution in amorphous silicon Schottky diodes [18] and thin $(<1$ $\mu \mathrm{m})$ pin solar cells.[19] Under reverse DC bias, when strongly absorbed light is incident on the $\mathrm{p}$-side ( $\mathrm{p}$-side generation), the electrons generated at the $\mathrm{p}$ - $\mathrm{i}$ interface drift toward the $\mathrm{n}$-layer under the decreasing electric field due to the space charge of the ionized dangling bonds. The induced photocurrent due to electron drift is

$$
I_{e}(t)=q N_{o} e^{-\frac{1}{\tau_{e}}} \frac{v_{e}(t)}{d}
$$

and the velocity is expressed by the following relation.

$$
v_{c}(t)=\frac{w}{\tau_{n}} e^{-\frac{1}{\tau_{n}}}
$$

Since from the equations (3.1) and (3.4)

$$
\tau_{\mathrm{n}}=\frac{\varepsilon_{\mathrm{o}} \varepsilon_{\mathrm{asi}}}{\mathrm{qN} N_{\mathrm{d}}^{*} \mu_{\mathrm{e}}}
$$


the induced current is an exponentially decreasing function of time

$$
L_{e}(t)=\frac{q w N_{0}}{d \tau_{n}} e^{-\left(\frac{1}{\tau_{e}}+\frac{1}{\tau_{n}}\right) t}
$$

and the ionized dangling bond density can be calculated from the slope in $\log (\mathrm{I})-\operatorname{lin}(\mathrm{t})$ plot by the following equation knowing $\mu_{e}$ and $\tau_{e}$.

$$
\text { Slope }=\left(\frac{q \mu_{\mathrm{e}}}{\varepsilon_{\mathrm{o}} \varepsilon_{\mathrm{asi}}}\right) N_{d}+\frac{1}{\tau_{\mathrm{e}}}
$$

\subsubsection{Drift Mobility and Lifetime}

The drift mobility and the lifetime of electrons and holes are generally measured by the transient photoconductivity method under pulsed-bias condition. This method is known as a pulsed-bias time-of-flight measurement. Fig. 3.4 shows the schernatic set-up for the time-of-flight method. Either the p- or the n-side of the pin diode is illuminated by short pulse of light with a strong absorption coefficient so that the electrons or holes are produced within $1 \mu \mathrm{m}$ of the appropriate contact soon after a square pulse of bias is applied. Depending on the direction of illumination, either electrons or holes generated around the $p-i$ or $n-i$ interfaces will drift under a uniform electric field $F$, and induce a transient photocurrent. By analyzing the shape of this induced photocurrent, the drift mobility and the lifetime of electrons or holes can be determined.

For such pulsed-bias measurements, it should be noted that; (a) The length of the bias pulse should be adjusted to be longer than the transir time of carriers and its repetition period should be larger than the dielectric relaxation time of the amorphous silicon, which is $\sim 100 \mu \mathrm{sec} .[20]$ (b) The intensity of the light should be small enough so that the external electric field will not be disturbed by the generated charges. When the light intensity is too high, the classical space charge limited current transient is observed with its characteristic cusp at the arrival time of the leading edge of the charges at the collecting contact.[21] 


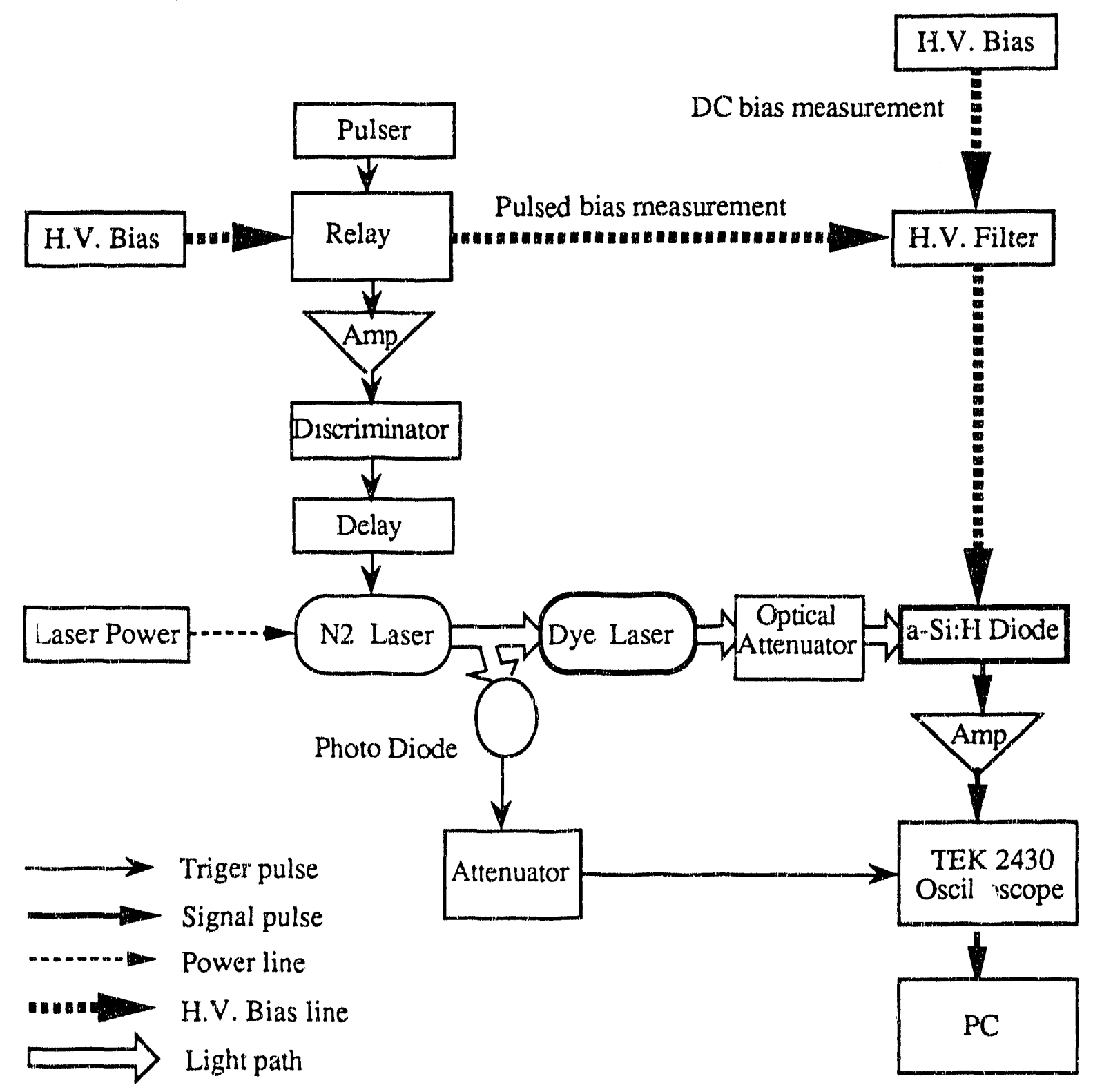

Fig. 3.4 A schematic diagram of experimental setup for time-of-flight measurement. Dye laser is pumped by $\mathrm{N}_{2}$ laser and produces light with the wave length $500 \sim 600$ $\mathrm{nm}$.

Since the electron transport is not dispersive at room temperature, the electron photocurrent is easier to analyze than that of the holes. Drift mobility is determined by measuring the transit time of carriers from one contact to the other. Because of carrier diffusion, the transit time is usually determined at the half value of maximum current 
intensity before decreasing as shown in Fig. 3.5. The average drift velocity is constant because the electric field is constant so the transit time is simply

$$
t_{t}=\frac{d}{v_{e}}=\frac{d}{\mu F}=\frac{d^{2}}{\mu V_{a}}
$$

Fig. 3.5 shows the electron time-of-flight data measured from a $27 \mu \mathrm{m}$ thick pin detector under three different biases. The electron transit times are marked by arrows.

The lifetime is determined by the rate of charge loss by trapping during the transit. If we assume $\mathrm{N}_{0}$ is the total generated charge, the remaining charge after time $t$ is simply expressed by the following equation using the definition of lifetime $\tau_{i}$.

$$
N(t)=N_{0} e^{\cdot \frac{1}{\tau_{i}}}
$$

Under the constant electric field, $F=V_{a} / d$, the induced photocurrent is

$$
I_{\text {ind }}(t)=\frac{q N(t) \mu F}{d}=\frac{q N_{0} e^{-t / \tau_{i}} \mu V_{a}}{d^{2}}
$$

The lifetime is usually estimated indirectly by the integral mode given by

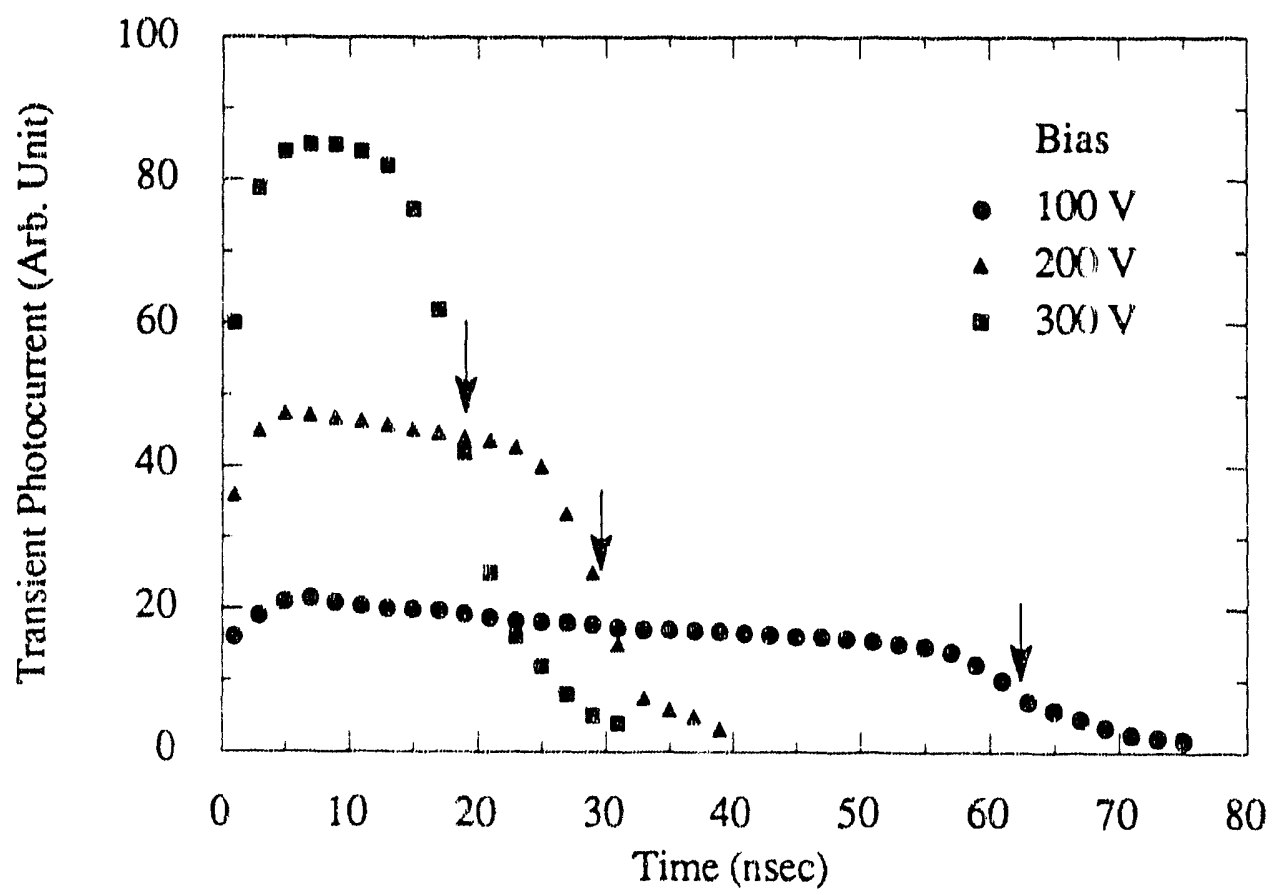

Fig. 3.5 Electron time-of-flight data for a $27 \mu \mathrm{m}$ thick a-Si:H pin diode. 


$$
Q\left(t_{i}\right)=\int_{0}^{t_{t}} I_{\text {ind }}(t) d t=Q_{0} \frac{\tau_{i}}{t_{t}}\left[1-e^{-w / \tau_{i}}\right]
$$

where $Q_{0}$ is $q N_{0}$ and the transit time $t_{t}$ is

$$
t_{t}=\frac{d^{2}}{\mu V_{a}}
$$

The above equation is called the Hecht equation.[22] Fig. 3.6 shows a plot of the measured integrated charge and a curve fit to the above equation to obtain the lifetime of electrons.

Holes have a very dispersive transport so the concept of drift mobility is less well defined. However the effective transit time of holes from one contact to another can be defined by an intersection point of two slopes in $\log (\mathrm{I})-\log (t)$ plot of hole photocurrent based on the dispersion model of Scher and Montroll.[23] In the model the transport is characterized by two slopes of $(1-\alpha)$ and $(1+\alpha)$ where $\alpha$ is called the dispersion parameter, and is given by $\alpha=T / T_{0}$ where $T$ is the temperature in degree Kelvin and $T_{0}$ is the inverse of the slope of the log of the density of valence-band tail states divided by the Boltzmann constant.[24]

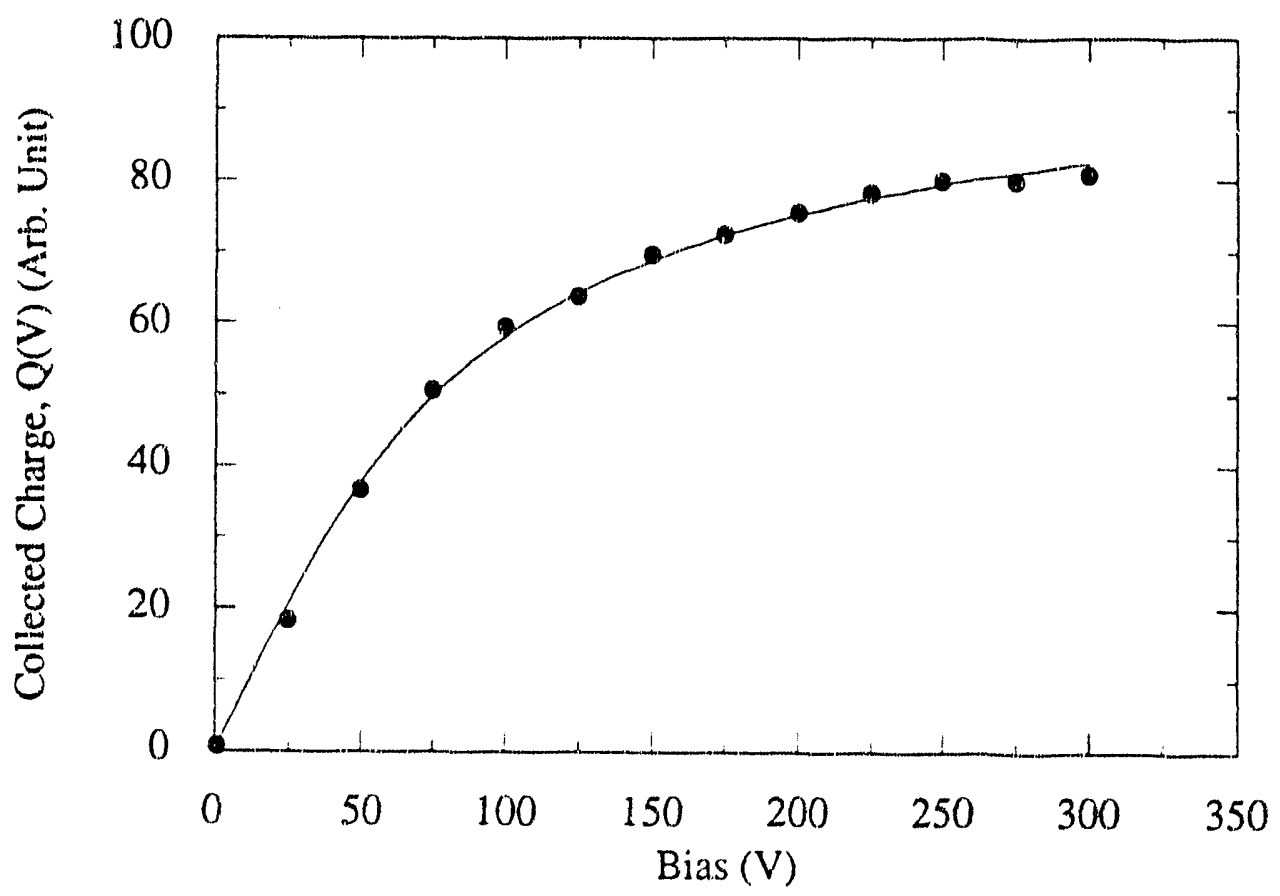

Fig. 3.6 Collected electron charges versus detector bias for a $27 \mu \mathrm{m}$ thick pin diode. 
Fig. 3.7 shows a typical hole transient photocurrent shape. The measured $\alpha$ of our sample diodes are in the range of $0.5 \sim 0.6$ at room temperature which is equivalent to the valence-band tail state width $40 \sim 50 \mathrm{meV}$. The lifetime of holes is also found from the Hecht equation. Fig. 3.8 shows the hole charge collection as a function of bias. Table 3.1 shows a list of measured parameters from sample diodes. From sample to sample in the same batch of diodes, data points have a fluctuation of $\sim 10 \%$ including the measurement error.

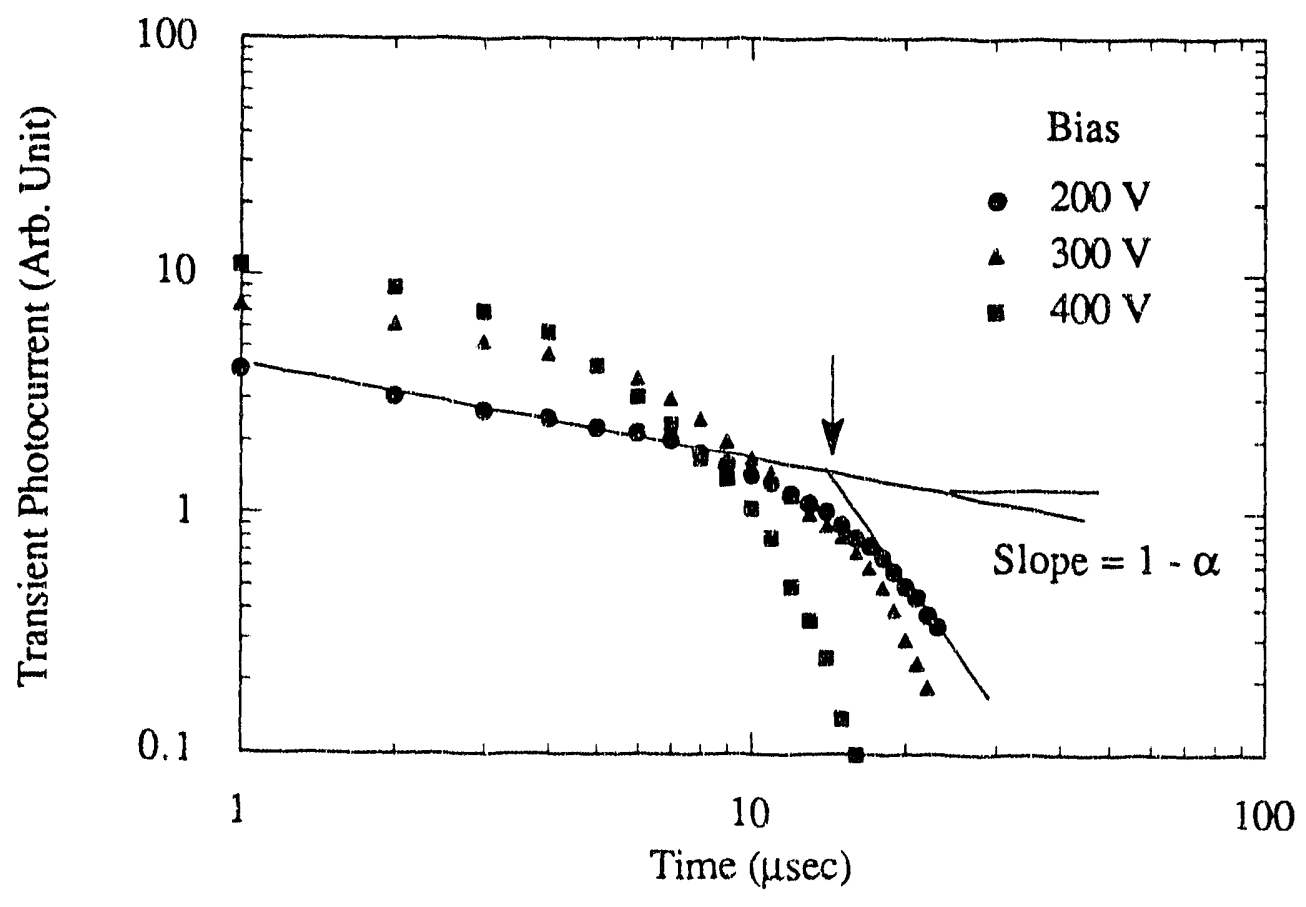

Fig. 3.7 Hole time-of-flight data for a $27 \mu \mathrm{m}$ thick a-Si:H pin diode. 


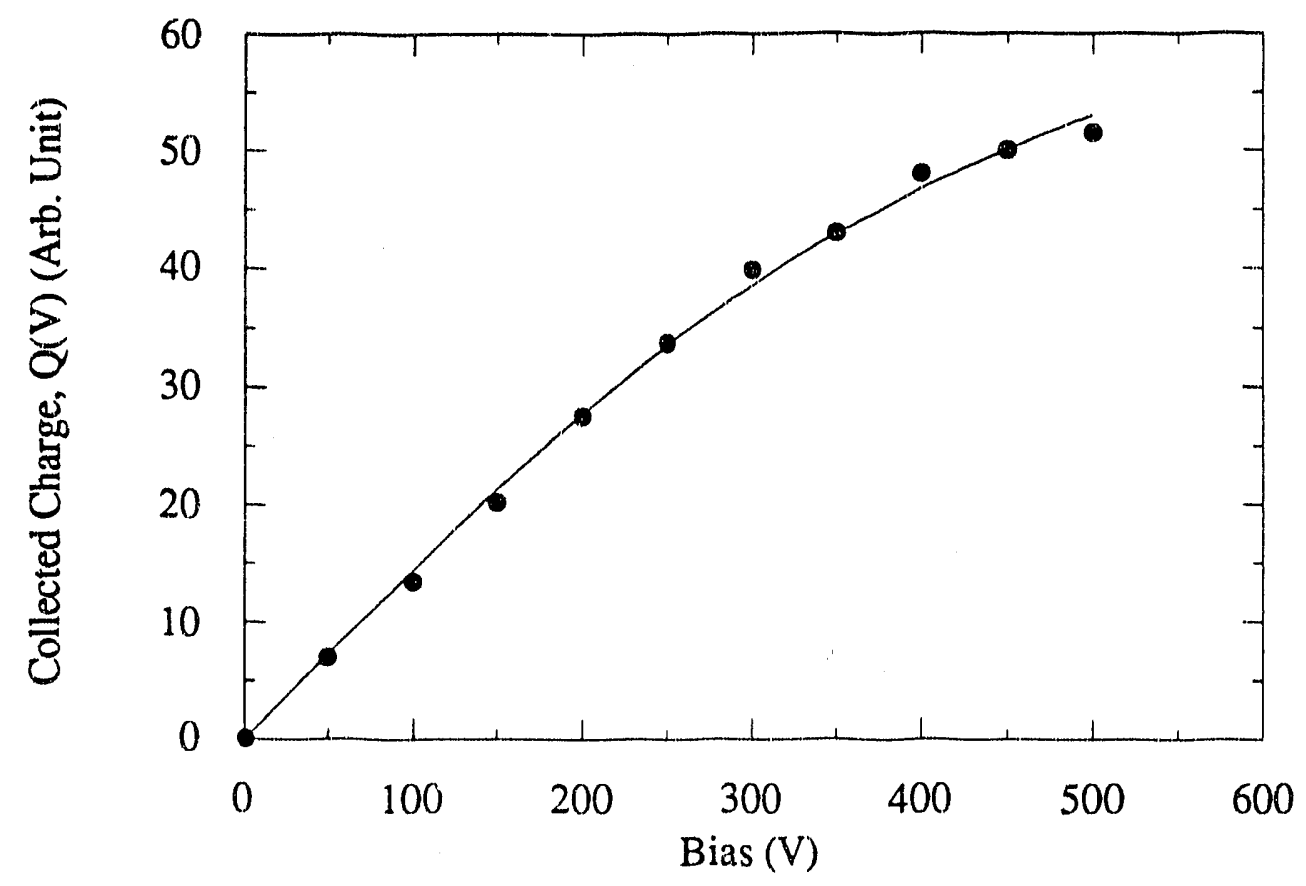

Fig. 3.8 Collected hole charges versus detector bias for a $27 \mu \mathrm{m}$ thick a-Si:H pin diode.

Table 3.1 Material parameters of a-Si:H pin diodes

\begin{tabular}{|c||c|c|c|c|c|c|}
\hline Thickness $(\mu \mathrm{m})$ & 5 & 12 & 12 & 27 & 28 & 48 \\
\hline Type $^{*}$ & $\mathrm{n}$-i-p & $\mathrm{n}$-i-p & $\mathrm{p}-\mathrm{i}-\mathrm{n}$ & $\mathrm{p}-\mathrm{i}-\mathrm{n}$ & $\mathrm{n}$-i-p & $\mathrm{n}$-i-p \\
\hline Maker & LBL & Xerox & GSI & GSI & Xerox & GSI \\
\hline$\mu_{\mathrm{e}}\left(\mathrm{cm}^{2} / \mathrm{Nsec}\right)$ & 1.0 & 1.1 & 1.2 & 1.2 & 1.4 & $0.6^{* *}$ \\
\hline$\tau_{\mathrm{e}}(\mathrm{sec})$ & $1 \times 10^{-7}$ & $1.3 \times 10^{-7}$ & $9.1 \times 10^{-8}$ & $8.1 \times 10^{-8}$ & $8.7 \times 10^{-8}$ & $1 \times 10^{-7}$ \\
\hline$\mu_{\mathrm{h}}\left(\mathrm{cm}^{2} / \mathrm{Vsec}\right)$ & 0.003 & 0.004 & 0.005 & 0.004 & 0.0035 & 0.003 \\
\hline$\tau_{\mathrm{h}}(\mathrm{sec})$ & $1.0 \times 10^{-6}$ & $4.0 \times 10^{-6}$ & $2.5 \times 10^{-6}$ & $3.0 \times 10^{-6}$ & $7.7 \times 10^{-6}$ & $<1 \times 10^{-7}$ \\
\hline $\mathrm{N}_{\mathrm{d}}{ }^{*}\left(10^{14} / \mathrm{cm}\right)$ & 7 & 6 & 7 & 7 & 6.5 & 7 \\
\hline
\end{tabular}

* Type means the deposition sequence starting from the first layer on the substrate.

** Data has not been verified and not used for averaging. 


\subsection{Calculation Results and Measurements}

\subsubsection{Calculation Results}

For calculations of charge collection efficiency we took the average values of measured parameters; $\mu_{\mathrm{e}}=1.2 \mathrm{~cm}^{2} / \mathrm{Vsec}, \mu_{\mathrm{h}}=0.004 \mathrm{~cm}^{2} / \mathrm{Vsec}, \tau_{\mathrm{e}}=9 \times 10^{-8} \mathrm{sec}, \tau_{\mathrm{h}}=3$ $\times 10^{-6} \mathrm{sec}, \mathrm{Nd}^{*}=7 \times 10^{14} / \mathrm{cm}^{3}$. Here we considered two cases of thick detector structure : (a) plain p-i-n detectors, and (b) multi-buried-layer detectors.

(1) Plain p-i-n detectors : Fig. 3.9 shows the calculated electric field distribution in a 70 $\mu \mathrm{m}$ thick pin diode using the two-region approximation. Two values of dangling bond density $3 \times 10^{14} / \mathrm{cm}^{3}$ and $7 \times 10^{14} / \mathrm{cm}^{3}$ are assumed. By reducing the dangling bond density the depletion region and thus charge collection efficiency increase and peak field drops by a factor the square root of the ratio of the decrease in dangling bond density.

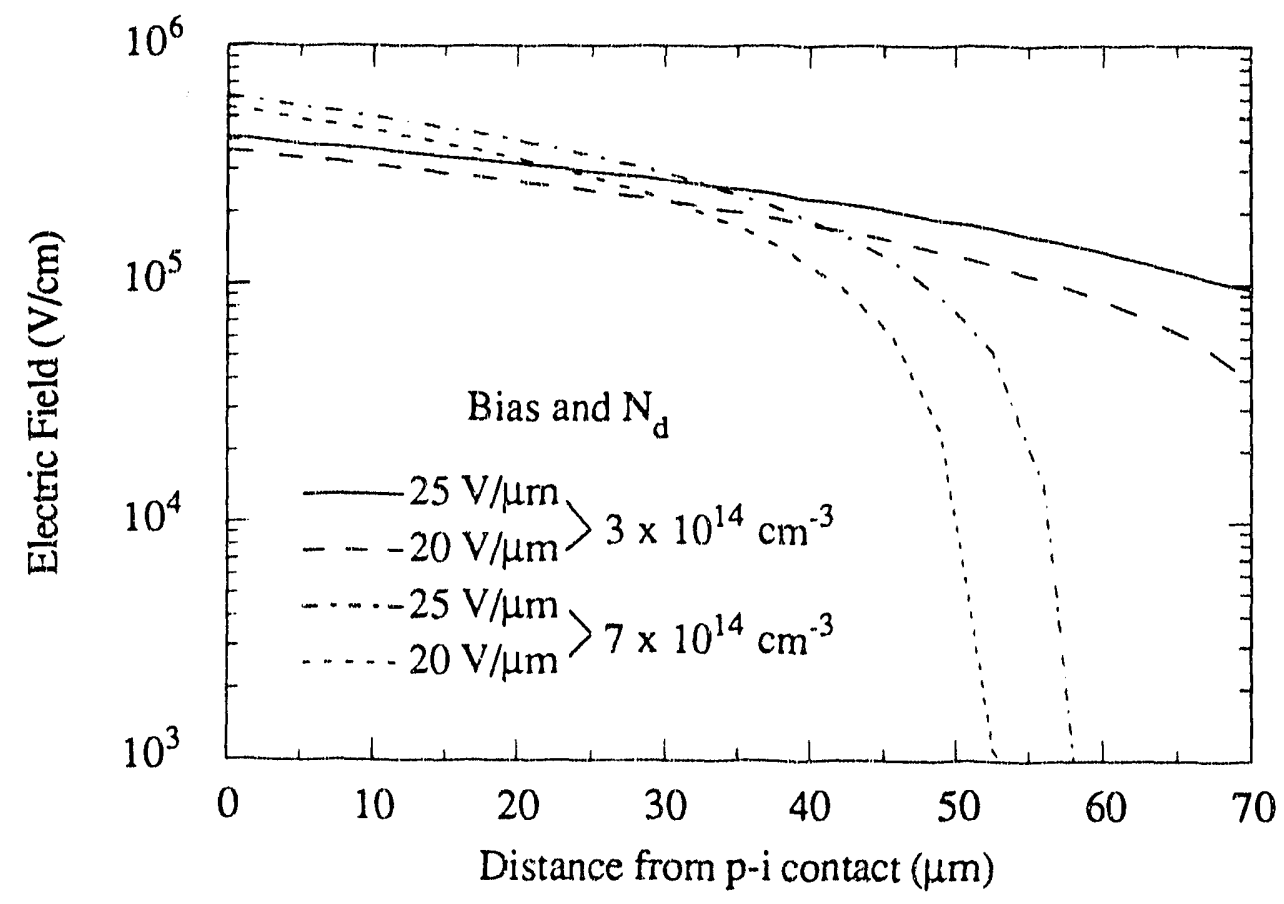

Fig. 3.9 Electric field in i-layer of a-Si:H pin diode calculated for two different biases and densities of ionized dangling bonds. 
Fig. 3.10 shows the calculated charge collection efficiency in the uniform generation case for diodes of thicknesses up to $50 \mu \mathrm{m}$ as a function of the bias at a shaping time of $1 \mu \mathrm{sec}$. Fig. 3.11 shows the calculated charge collection efficiency for a-Si:H pin diodes as a function of the shaping time at three different biases. The applied biases are normalized to the detector thickness to keep the same depletion parameter Y. Fig. 3.12 shows the calculated output signal pulse shape of $C R-(R C)^{n}$ shaping amplifier with $n=1 \sim$ 4 at a shaping time of $1 \mu \mathrm{sec}$.

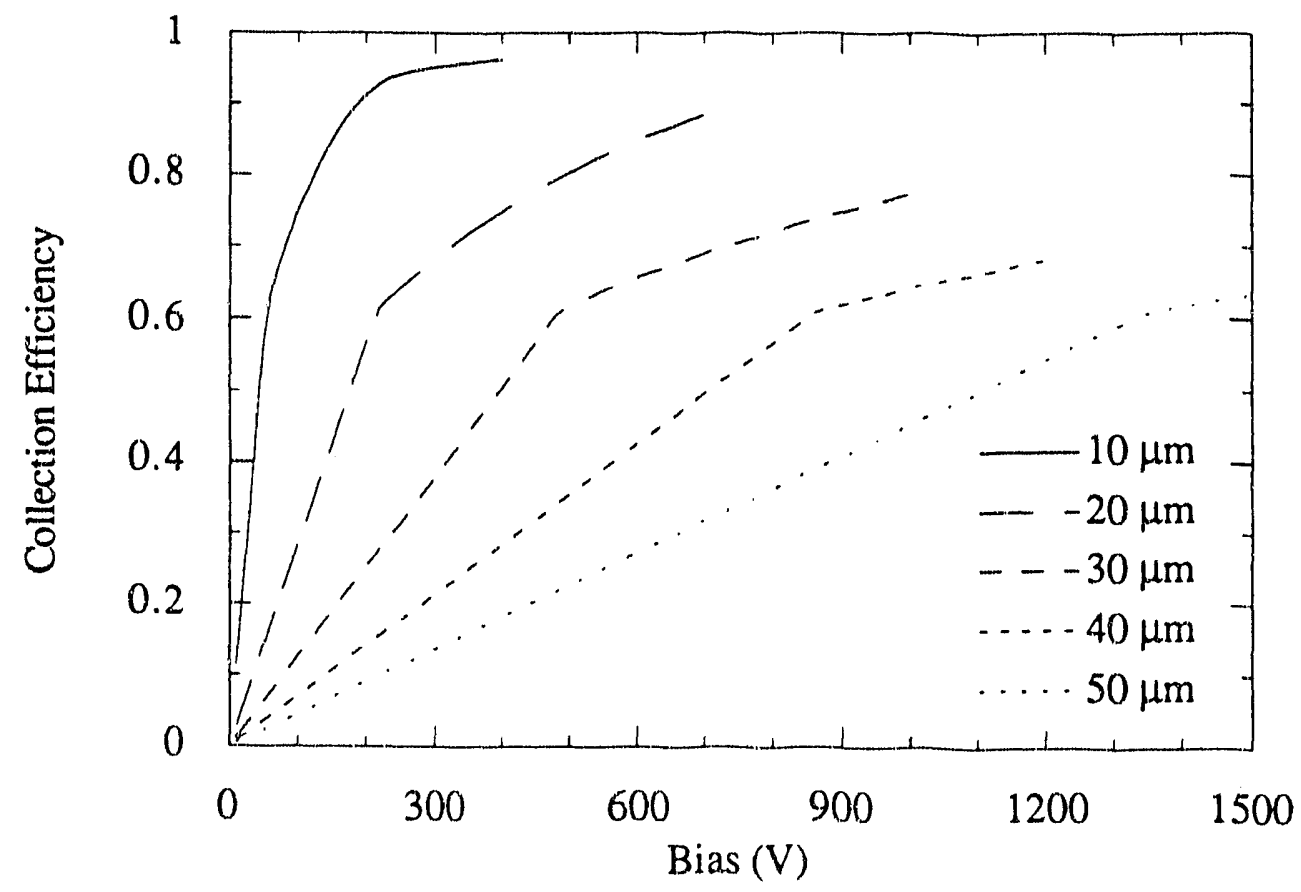

Fig. 3.10 Calculated charge collection efficiency in detectors of the thickness up to $50 \mu \mathrm{m}$ at a fixed shaping time of $1 \mu \mathrm{sec}$. 


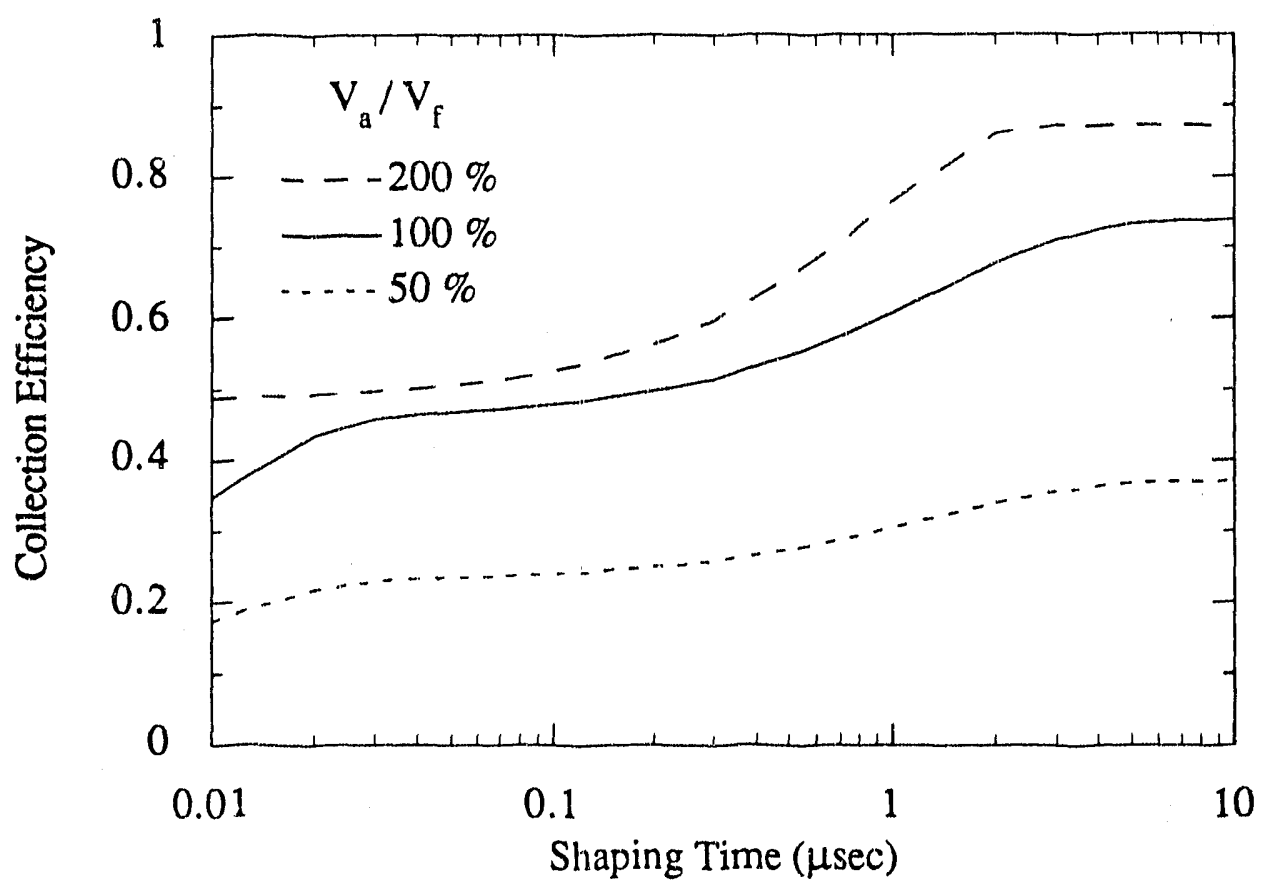

Fig. 3.11 Calculated charge collection efficiency in a-Si:H pin detectors as a function of shaping time for three different biases. $n=1$ was used.

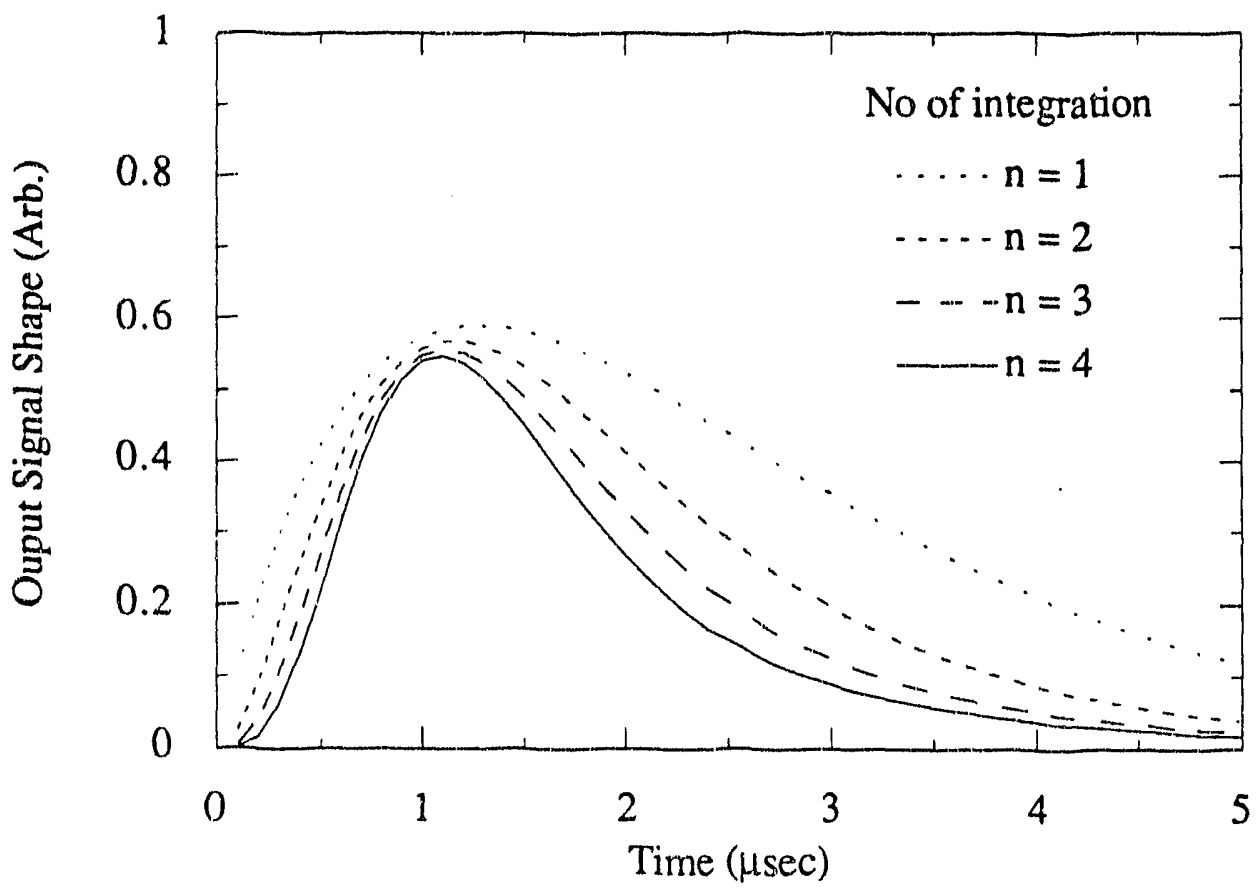

Fig. 3.12 Calculated output signal pulse shapes of $C R-(R C)^{n}$ shaping amplifier with $n=1$ $\sim 4$ at $1 \mu \mathrm{sec}$ shaping time and a full depletion bias $(\mathrm{Y}=1)$. 
(2) Multi-Buried-Layer Structure : In order to reduce the bias necessary to deplete thick detectors either the ionized dangling bond density should be reduced by improving the deposition technique or the slightly n-type intrinsic layer can be compensated by doping borons with an equal density to that of dangling bonds. Better control can be achieved by deposition of thin slightly doped p-layers in the i-layer.

Fig. 3.13 shows a schematic picture of the electric field configuration in $50 \mu \mathrm{m}$ thick detectors with and without an inserted p-layer in the middle of the i-layer. In the latter scheme the bias required to deplete the total i-layer is decreased by a factor of two if the buried middle p-layer builds negative space charges which compensate the positive charges of ionized dangling bonds in the second half of the i-layer. This can be done by controlling the thickness and doping level of the buried layer by changing the $\mathrm{B}_{2} \mathrm{H}_{6}$ gas concentration in $\mathrm{SiH}_{4}$.[25] This scheme also reduces the peak electric field at $\mathrm{p}-\mathrm{i}$ contact by the same factor. Inserting more buried p-layers can reduce the depletion bias further.

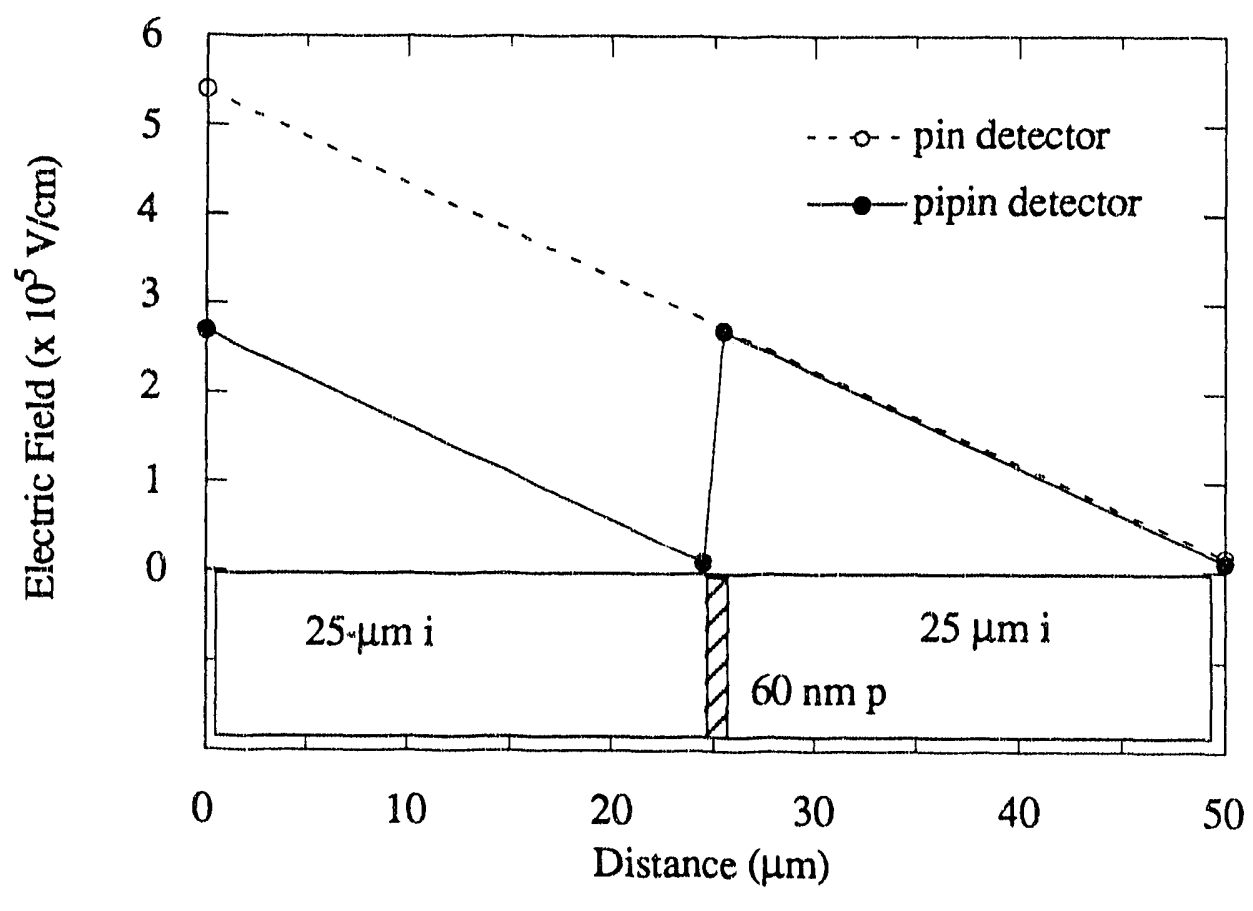

Fig. 3.13 Calculated electric field of $50 \mu \mathrm{m}$ pin and buried pipin detectors under the reverse bias of $1400 \mathrm{~V}$ and $700 \mathrm{~V}$ respectively. Insertion is a schematic of pipin detector structure. Assumed ionized dangling bond density is $7 \times 10^{14} / \mathrm{cm}^{3}$. 
A calculation of the electron collection efficiency in a multi-buried-layer detector is given in Appendix B. Since holes have very low mobility and the detectors of interest are thick $(>50 \mu \mathrm{m})$, the hole collection efficiency is neglected. Fig. 3.14 shows the electron collection efficiency as a function of the number of buried p-layers in a $50 \mu \mathrm{m}$ thick detector with bias of $675 \mathrm{~V}$, which could only deplete a $35 \mu \mathrm{m}$ thick plain pin detector.

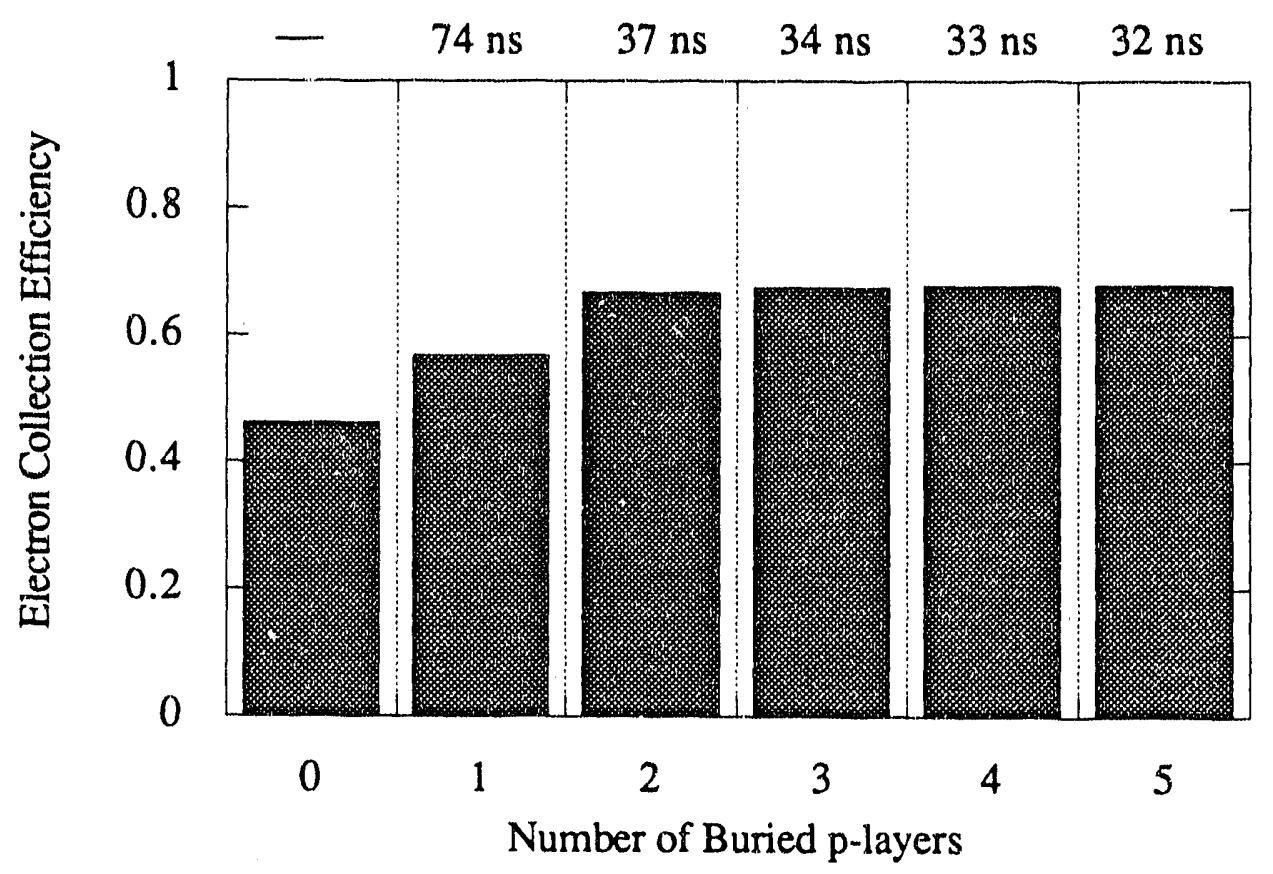

Fig. 3.14 Calculated electron collection efficiency as a function of the number of the buried p-layers in a $50 \mu \mathrm{m}$ thick detector at a fixed bias of $675 \mathrm{~V}$. The number above the bar is the transit time. 


\subsubsection{Signal Measurements}

(1) Simulation by Light Sources :

In order to simulate the charge collection process, light sources (LED or laser) with two wavelengths are used. LED light with a wavelength of $760 \mathrm{~nm}$ has a long mean free path $(1 / \alpha=\sim 100 \mu \mathrm{m})$ so it can simulate the uniform generation case, and $510 \mathrm{~nm}$ laser light simulates the surface generation case because of its short mean free path $\sim 0.2 \mu \mathrm{m}$.

Fig. 3.15 and Fig. 3.16 show the measured data points of the collection efficiency for a $760 \mathrm{~nm}$ light source on a $27 \mu \mathrm{m}$ thick sample diode as a function of the bias and of the shaping time together with the calculated values using the equations derived in section 3.2. Fig. 3.17 shows the measured collection efficiency for $510 \mathrm{~nm}$ light incident on the same sample. In the case of n-side illumination, the hole signal is zero until the full depletion bias, $350 \mathrm{~V}$ for this sample, is applied as explained in the section 3.3.2.

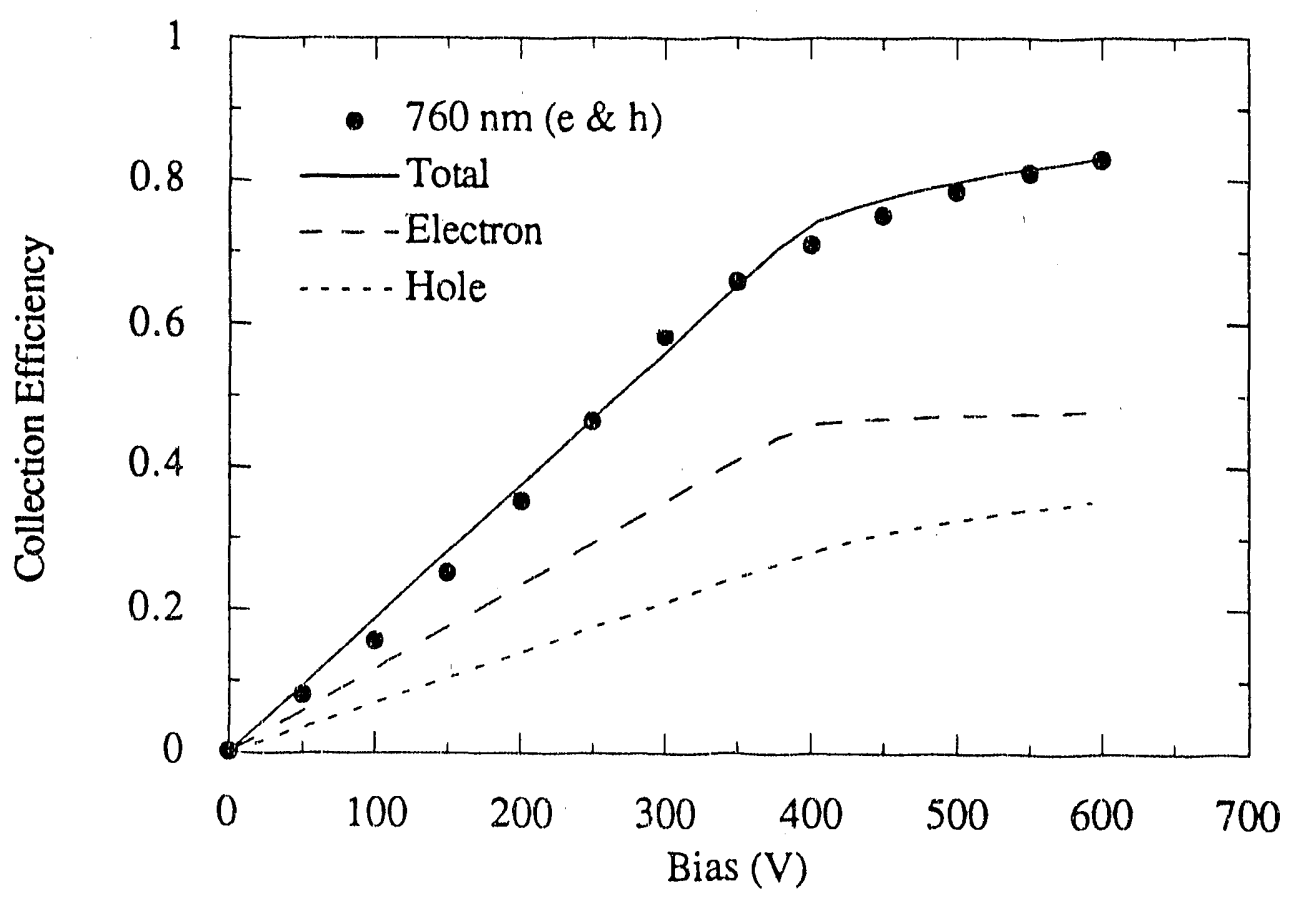

Fig. 3.15 Collection efficiency vs bias of a $27 \mu \mathrm{m}$ thick sample for $760 \mathrm{~nm}$ light. Dots are measured data and lines are calculation results. Shaping time used is $9 \mu \mathrm{sec}$. 


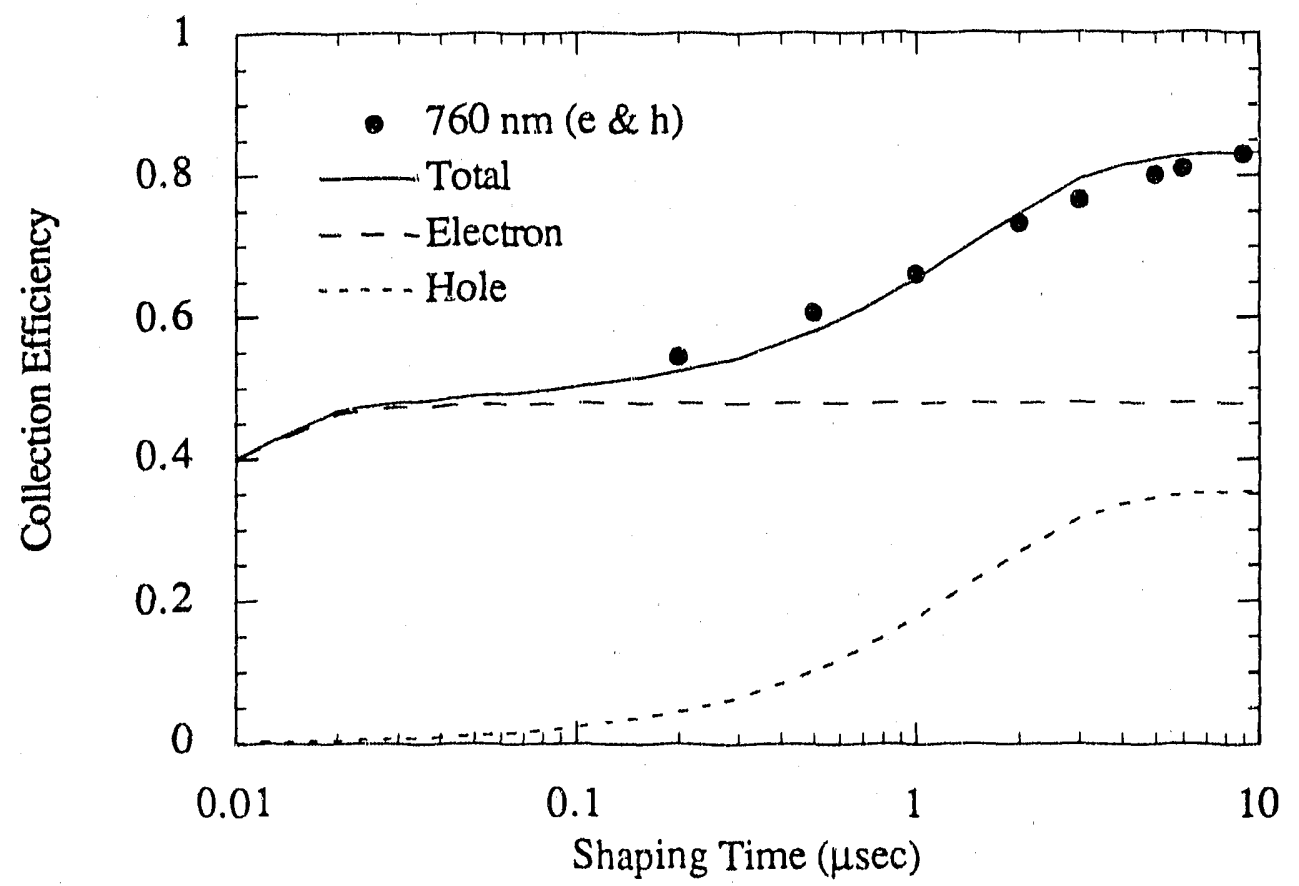

Fig. 3.16 Collection efficiency vs shaping time of a $27 \mu \mathrm{m}$ thick sample for $760 \mathrm{~nm}$ light. Dots are measured data and lines are calculation results. Applied bias is $600 \mathrm{~V}$.

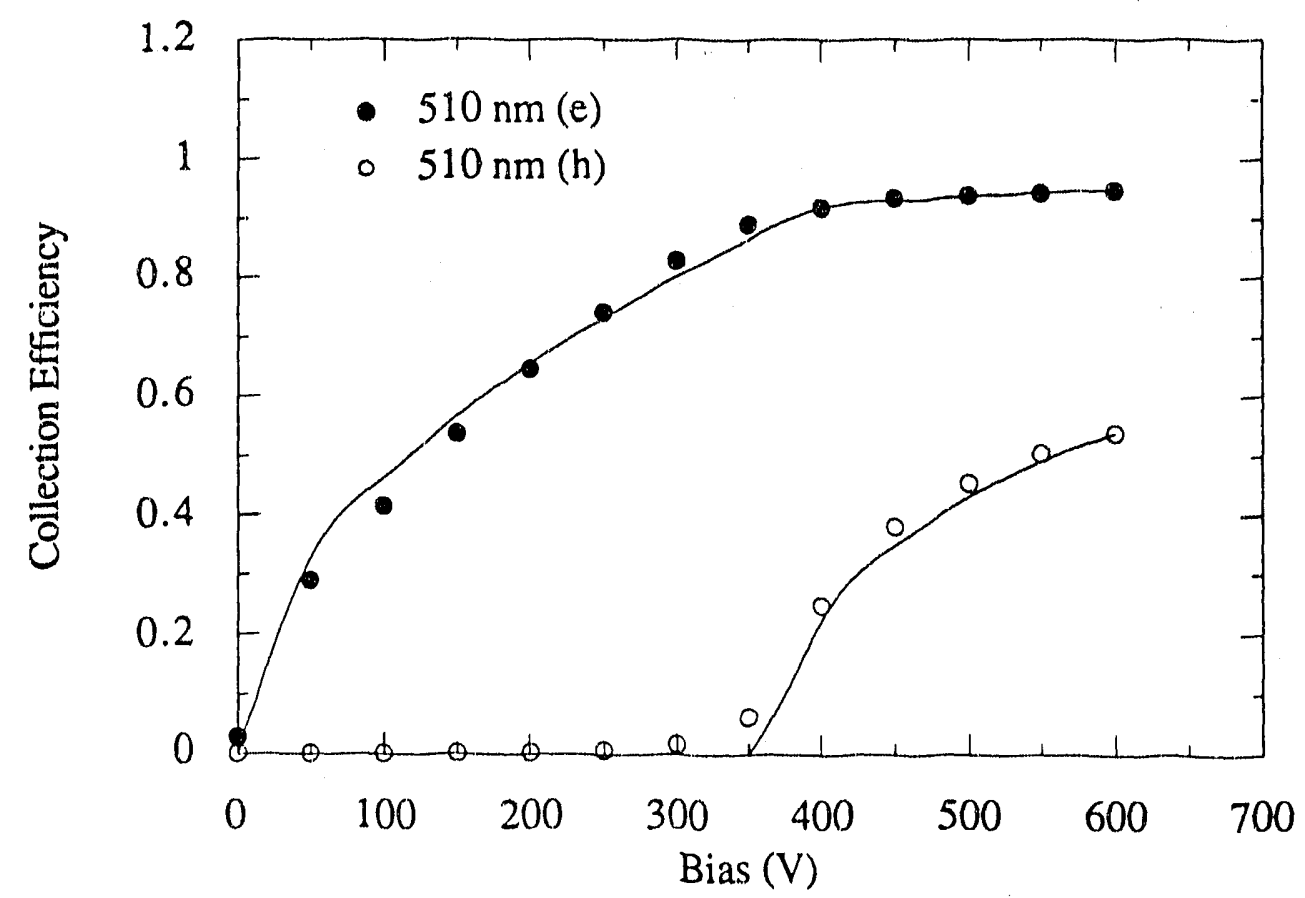

Fig. 3.17 Collection efficiency of electrons and holes in the cases of surface generation for a $27 \mu \mathrm{m}$ thick sample. Dots are measured data from $510 \mathrm{~nm}$ laser light and lines are calculation results. 
(2) Minimum Ionizing Beta Particle Measurement:

In Fig. 3.18 we show the measured signal produced by $1 \mathrm{MeV}$ betas from $\mathrm{Sr}^{90}$ incident on a $27 \mu \mathrm{m}$ thick diode. Since the sample diodes produce larger noise than the signal due to the large capacity of the contact area, the signals produced were measured by a pulse averaging method. It has been reported.[14] $1 \mathrm{MeV}$ electrons have a range of $\sim 230$ $\mu \mathrm{m}$ in crystalline silicon [26] and $\Delta \mathrm{E}(=\mathrm{dE} / \mathrm{dx})$ is $\sim 300 \mathrm{eV} / \mu \mathrm{m}$, so it can be considered as a uniform generation case. Calculation used the measured parameters for this sample and the result fits well to the measured data.

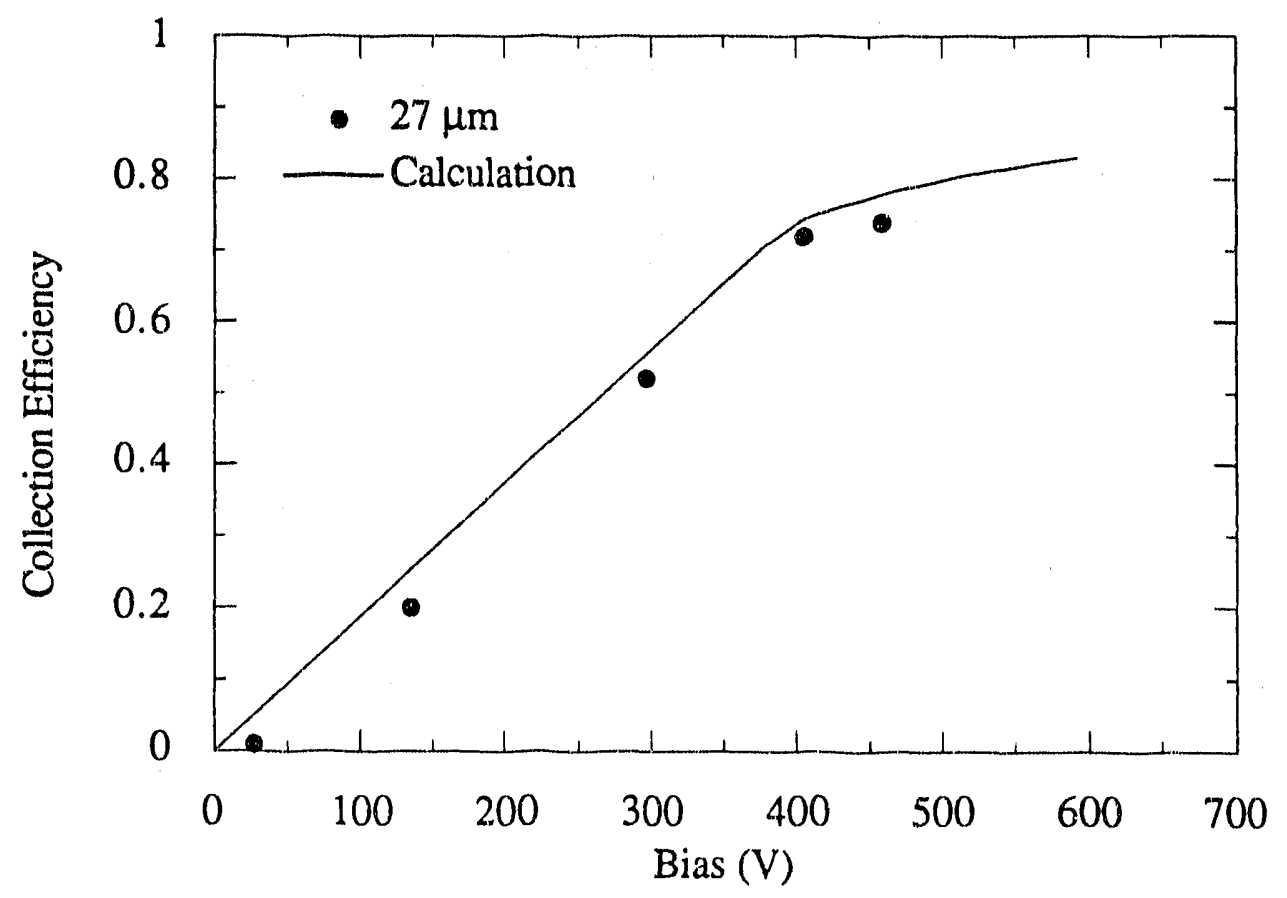

Fig. 3.18 Signal collection efficiency produced by $1 \mathrm{MeV}$ betas from $\mathrm{Sr}^{90}$ incident on a diode of thickness $27 \mu \mathrm{m}$. Shaping time used was $2.5 \mu \mathrm{sec}$. 


\subsection{Discussion}

The analytical form of the charge collection efficiency derived based on several assumptions fits very well to the measurements of uniform generation and surface generation cases simulated by various wave length light and minimum ionizing beta particles.

The implication of these calculations and measurements is that a-Si:H pin detectors of thickness less than $5 \mu \mathrm{m}$ can collect almost all the electrons and holes at a shaping time of $1 \mu \mathrm{sec}$. The measured charge collection in a $27 \mu \mathrm{m}$ thick detector were $95 \%$ of the electrons at a shaping time less than $0.2 \mu \mathrm{sec}$ and $70 \%$ of the holes at $5 \mu \mathrm{sec}$, agreed well with the calculation results. The calculation showed that a $50 \mu \mathrm{m}$ thick detector can collect $90 \%$ of the electrons at $20 \mathrm{nsec}$ and $25 \%$ of the holes at $5 \mu \mathrm{sec}$ at the full depletion bias. The latter is equivalent to $\sim 60 \mathrm{e}-\mathrm{h}$ pairs/ $\mu \mathrm{m}$ or $\sim 3000$ electrons combining the $\mathrm{W}$-value of $5 \mathrm{eV}$. Calculation also shows that detectors with different thickness have the equal charge collection efficiency as long as the bias is applied to keep the same depletion thickness.

Thicker detectors require a larger bias to deplete the whole i-layer. Because of this, the diodes have a very high peak field at the p-i junction which can cause the breakdown [27]. This breakdown as well as stress set a practical limit in making thick detectors. A multi buried layer scheme can reduce the high peak field problem. Calculations showed that the more p-layers are inserted, the higher collection efficiency is achieved at a same bias. However more than three inserted p-layers do not give larger benefits.

Another way of solving the high peak field problem due to large bias is to make the sample with lower dangling bond densities. Reduction it the dangling bond density by a factor of 2 can yield in a drop of the peak field to $70 \%$ and an improvement cf charge collection efficiency by $40 \%$ in a $50 \mu \mathrm{m}$ thick detector. 


\section{References}

[1] S. Ramo, "Currents Induced by Electron Molion," Proc. I.R.E., Vol. 27, 584 (1939)

[2] P. A. Tove and K. Falk, "Pulse Formation and Transit Time of Charge Carriers in Semiconductor Junction Detectors," Nucl. Instr. and Meth., 29, 66 (1964)

[3] M. Hack, S. Guha and M. Shur, "Photoconductivity and Recombination in Amorphous Silicon Alloys," Phy. Rev. B, Vol. 30, 6991 (1984)

[4] S. Guha and M. hack, "Dominant Recombination Process in Amorphous Silicon Alloy," J. Appl. Phys., 58, 1683 (1985)

[5] A. Alberigi Quaranta, A. Taroni, and G. Zanarini, "Plasma Time and Related Delay Effects in Solid State Detectors," Nucl. Instr. and Meth., 72,72 (1969)

[6] E. C. Finch and A. L. Rodgers, "Measurements of the Pulse Height Defect and its Mass Dependence for Heavy-ion Silicon Detectors," Nucl. Instr. and Meth., 113, $29(1973)$

[7] S. Qureshi, V. Perez-Mendez, S. N. Kaplan, I. Fujieda, G. Cho, and R. A. Street, "Material Parameters in a Thick Hydrogenated Amorphous Silicon Detector and Their Effect on Signal Collection," Mat. Res. Soc. Symp. Proc., Vol. 149, p. 649 (1989)

[8] J. P. Kleider, C. Longeaud, O. Glodt, D. Mencaraglia and R. Vanderhaghen, "Field-profile Determination in Arnorphous Si-Ge Alloy Schottky Barriers," Phil. Mag. B, Vo. 64, 367 (1991)

[9] E. Mathieson and M. W. Charles, "Attneuation of Proportional Counter Pulses by Equal Integrating and Differentiating Time Constants," Nucl. Instr. and Meth., 72 , 155 (1969)

[10] P. W. Nicholson, Nuclear Elecironics, John Wiley \& Sons Inc., p. 179 (1974)

[11] F. S. Goulding and D. A. Landis, "Signal Processing for Semiconductor Detectors," IEEE Trans. Nuc. Sci., NS-29, 1125 (1982)

[12] G. F. Knoll, Radiation Detection and Measurement, John Wiley \& Sons Inc., p. $623(1979)$

[13] W. J. Price, Nuclear Radiation Detection, McGraw-Hill Inc., p. 383 (1958)

[14] S. N. Kaplan, I. Fujieda, V. Perez-Mendez, S. Qureshi, W. Ward and R. A. Street, "Detection of Minimum Ionizing Particles in Hydrogenated Amorphous Silicon," Nucl. Instr. and Meth., A273, 611 (1988)

[15] S. Qureshi, Hydrogenated Amorphous Silicon Radiation Detectors: Material Parameters; Radiation Hardness; Charge Collection, Ph.D. Thesis, UC Berkeley (1991) 
[16] C. A. Klein, "Band Gap Dependence and Related Features of Radiation Ionization Energies in Semiconductors," J. Appl. Phys., 39, 2029 (1968)

[17] S. Qureshi, V. Perez-Mendez, S. N. Kaplan, I. Fujieda, G. Cho and R. A. Street, "Material Paranieters in Thick Hydrogenated Amorphous Silicon Radiation Detectors," J. Non-Crystalline Solids, Vol. 114, 417 (1989)

[18] R. A. Street, "Measurement of Depletion Layers in Hydrogenated Amorphous Silicon," Phy. Rev. B, Vol. 27, 4924 (1983)

[19] R. Konenkamp, S. Muramatsu, H. Ito, S. Matsubara and T. Shimada, "Determination of Electric Field Profiles in Amorphous Silicon Solar Cells," Appl. Phys. Lett., 57, 478 (1990)

[20] T. Tieje, B. Abeles, D. L. Morel, T. D. Moustakas and C. R. Wronski, "Electron Drift Mobility in Hydrogenated a-Si," Appl. Phys. Lett. 36, 695 (1980)

[21] T. Tieje, C. R. Wronski, B. Abeles and J. M. Cebulka, "Electron Transport in Hydrogenated Amorphous Silicon: Drift Mobility and Junction Capacitance," Solar Cells, 2, 301 (1980)

[22] R. Konenkamp, A. M. Hermann and A. Madan, "Measurement of Mobility lifetime Product in Hydrogenated Amorphous Silicon p-i-n Type Diodes," Appl. Phys. Lett., 46, 405 (1985)

[23] H. Scher and E. W. Montroll, "Anomalous Transit-time Dispersion in Amorphous Solids," Phys. Rev. B, Vol. 12, 2455 (1975)

[24] G. Pfister and H. Scher, "Dispersive (Non-Gaussian) Transport in Disordered Solids," Adv. Phys., Vol. 27, 747 (1978)

[25] M. Siutzmann, D. K. Biegelsen and R. A. Street, "Detailed Investigation of Hydrogenated Amorphous Silicon and Germanium," Phys. Rev., B35, 5666 (1987)

[26] T. Mukoyama, "kange of Electrons and Positrons," Nucl. Instr. Meth., 134, 125 (1974)

[27] R. A. Gibson, P. G. LeComber and W. E. Spear, "The Characteristics of High Current Amorphous Silicon Diodes," Appl. Phys., 21, 307 (1980) 


\section{Chapter 4 Electronic Noise in a-Si:H Detectors}

\subsection{Introduction}

The sensitivity of detection and resolution of the radiation image is limited by the total noise of the detection system which consists of two components combining in quadrature, statistical noise, $\mathrm{N}_{\text {sta }}$, and system noise, $\mathrm{N}_{\text {sys. }}$ (Noise in a radiation detection system is most conveniently expressed in unit of electrons in order to compare directly with the signal charge, $S_{\text {sig.) }}$

$$
N_{\text {tot }}^{2}=N_{\text {sta }}^{2}+N_{\text {sys }}^{2}
$$

The statistical noise, $N_{\text {sta }}$, is due to a statistical fluctuation in the number of electron-hole pairs produced by the interaction in the radiation detector. The interaction follows a modified Poisson statistics and its variance is proportional to the signal charge, $S_{s i g}$, the average number of e-h pairs collected. The statistical noise may be expressed as

$$
\mathrm{N}_{\text {sta }}^{2}=\mathrm{F} \times \mathrm{S}_{\text {sig }}
$$

where $F$ is called the Fano factor [1], and is defined as the ratio of the observed variance to the theoretical variance in the charge generation process. F is usually less than unity due to the fact that the charge generation is not purely random, for example, $\mathrm{F} \approx 0.1$ for $\mathrm{Si}$ and $\mathrm{F}$ $\approx 0.06$ for $\mathrm{Ge}$.[2] The importance of the statistical noise decreases as the signal size increases because the signal to noise ratio is inversely proportional to the square root of the signal size.

The system noise, $\mathrm{N}_{\text {sys }}$, originates from the detector and the signal-pulseprocessing electronics, such as the preamplifier, shaping amplifier, ADC etc. Since ine detector and the front-end stage of the preamplifier are the main contributors to the overall system noise, this noise can be rewritten as

$$
N_{\text {sys }}^{2}=N_{d c t}^{2}+N_{a m p}^{2}
$$

An accurate formulation of the electronic noise sources is very important in the design of low noise radiation detection system with high sensitivity and large signal-to-noise ratio. 
The electronic noise from reverse-biased a-Si:H pin diodes and a-Si:H and poly-silicon thin-film-transistors will be discussed in this Chapter. The equivalent noise charge from a$\mathrm{Si}: \mathrm{H}$ pin detectors will be measured and analyzed to obtain readily-usable expressions of detector noise components for the optimum design of large-area pixel detector systems.

\subsection{Noise of a-Si:H Detectors}

Amorphous silicon radiation detectors are reverse-biased pin diodes or Schottky diodes in which the $\mathrm{i}$-layer is slightly $\mathrm{n}$-type. Therefore it behaves like $\mathrm{p}-\mathrm{n}$ junction diodes. However, because of the high density of gap states, the reverse current and noise characteristics are quite different form those of crystalline silicon $p$ - $n$ diodes.

\subsubsection{Reverse Current in a-Si:H pin Diode}

In crystalline $p-n$ junction diodes or Schottky-barrier diodes at low to moderate bias the current is

$$
\mathrm{I}(\mathrm{V})=\mathrm{I}_{\mathrm{R}}(\mathrm{V})[\exp (\mathrm{qV} / \mathrm{kT})-1]
$$

where $V$ is the applied bias and $I_{R}(V)$ is the reverse saturation current which is a weak function of the bias. Under reverse bias $|\mathrm{V}| \gg \mathrm{kT} / \mathrm{q}$, the current becomes $-I_{R}(V)$. The reverse saturation current $I_{R}$ has two sources; (a) barrier injection and (b) thermal generation in the depleted region. Sometirnes in a poor diode extra current due to surface leakage is observed.

In the ideal semiconductor diode without the generation, $I_{R}$ is constant and is purely due to the barrier injection, which is calculated by diffusion theory in a $p-n$ junction diode [3] and by thermionic emission-diffusion theory in a Schottky diode. [4] However, in most crystalline diodes the thermal generation of electrons and holes in the depleted region is the dominant current source under reverse bias condition. With a single-level recombination 
center, $I_{R}$ has been calculated by Sah et al.,[5] using the Shockley-Read-Hall (SRH) model, [6] to be proportional to $\sqrt{\mid V}$ under reverse bias.

We shall make several assumptions for modelling the I-V characteristics in a reverse biased thick a-Si:H pin diode. These are; (a) metal to n- and p-layer contacts make good ohnye corntacts for the majority carriers and blocking contacts for the minority carriers. (b) the $\mathrm{i}-\mathrm{n}$ interface is a good ohmic contact for electrons. (c) the minority carrier density in both $\mathrm{p}$ - and $\mathrm{n}$ - layers are totally negligible. These assumptions are generally acceptable because the $n$ - and p-layers are heavily doped and quite thin. (d) Direct generation from the valence band to the conduction band is neglected because of the large band gap energy of aSi. Finally, (e) the most important interface is the p-i contact, and we shall consider the player as a metal layer which makes an effective Schottky contact to the i-layer in terms of electron injection.

In most conditions of operation at reverse bias, the reverse current is due to the generation of charge carriers in the bulk $i$-layer and to injection of electrons at the p-i interface through multi-step tunneling, called hopping. Fig. 4.1 is a schematic of the band structure of a reverse-biased pin diodes showing two current sources.

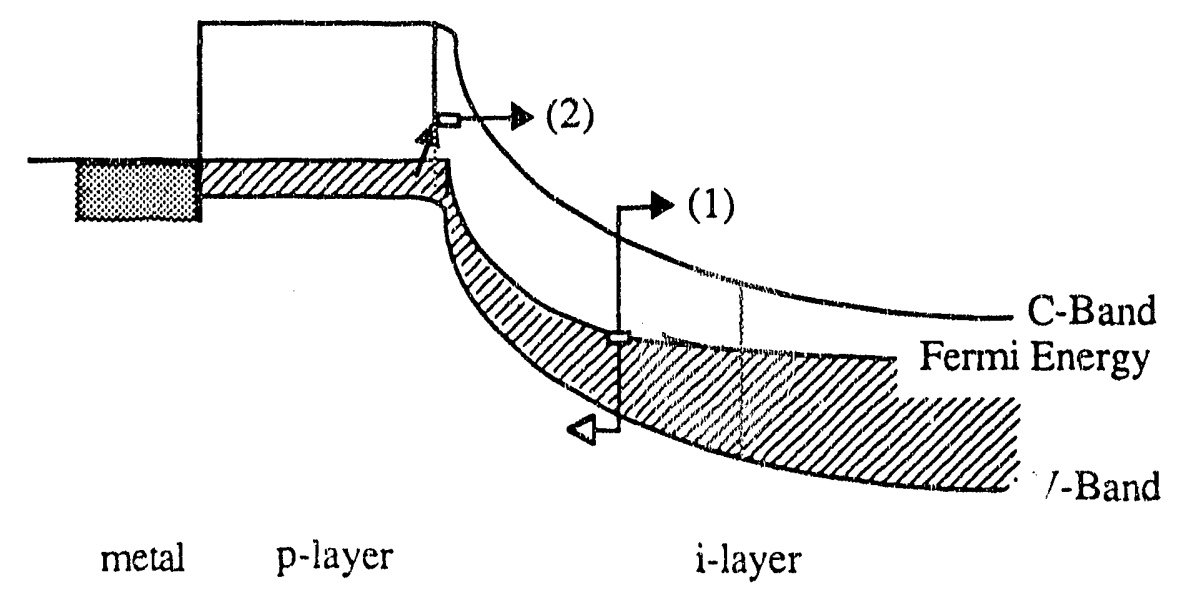

Fig. 4.1 Two sources of current in reverse-biased a-Si:H pin diodes. (1) Electron-hole pair generation through a mid-gap state and (2) electron tunneling injection from the $\mathrm{p}-\mathrm{i}$ interface through multistep hopping. 


\section{(1) Bulk Generation Current:}

The generation-recombination rate is usually calculated by the SRH model, Under reverse bias, recombination is usually negligible compared to generation because of low free carrier densities in the space charge region. The thermal generation rate from a single recombination center is given by

$$
e_{0}=\omega_{0} \exp \left[-\left(E_{c}-E_{l}\right) / k T\right]
$$

where $E_{c}$ and $E_{t}$ are respectively the energy level of the conduction band edge and of a recombination center (also called a generation center). The emission rate prefactor $\omega_{0}$ is typically $10^{12} \sim 10^{13} \mathrm{sec}^{-1}$ in a-Si:H.[7] The total generation current from distributed recombination centers in the bulk $\mathrm{i}-\mathrm{layer}, \mathrm{I}_{\mathrm{BG}}$, may be obtained from the following integral,

$$
I_{B G}=q A \int_{0}^{d} d x \int_{E_{v}}^{E_{c}} N(E, x) f_{t}\left(E, E_{f}, x\right) e_{0}(E, x) d E
$$

where $A$ is the contact area, $d$ is the thickness of the i-layer. $N(E, x)$ is the density of recombination centers per unit volume at $x$ and per unit energy at $E$, and $f_{t}$ is the occupation probability of the recombination center (i.e., the Fermi distribution function). In order to generate an equal number of electrons and holes, the Fermi energy is close to halfway between the valence and conduction band edges. Carrier emission is only significant from recombination centers within $\mathrm{kT}$ of the Fermi energy level, so the current can be written approximately as

$$
\int_{E_{f}-k T}^{E_{f}} N(E, x) f_{t}(E) d E \approx N_{t} k T
$$

Therefore the above equation becomes

$$
I_{B G}=q A \int_{0}^{d} N_{l}(x) k T \omega_{0} \exp \left[-E_{i}(x) / k T\right] d x
$$

where $N_{l}(x)$ is the density of states near the Fermi energy level and $E_{i}(x)$ is the thermal barrier $\left(=E_{\mathcal{c}}-E_{f}=E_{c}-E_{\mathfrak{l}}\right)$. The above equation depends on the $x$ variable only and is easy to apply to non uniform generation cases such as highly reverse biased a-Si:H pin diodes. 
If the recombination centers are uniformly distributed in space, the bulk generation current is given by

$$
\mathrm{I}_{\mathrm{BG}} \approx \mathrm{qAdN} \mathrm{N}_{\mathrm{t}} \mathrm{kT} \omega_{\mathrm{o}} \exp \left[-\mathrm{E}_{\mathrm{i}} / \mathrm{kT}\right] \equiv \mathrm{qAdN_{ \textrm {t } }} \mathrm{kT} \mathrm{e}_{\mathrm{TH}} \equiv \mathrm{I}_{\mathrm{TH}}
$$

where $e_{T H}$ is the thermal emission rate from the Fermi energy level.

This simple result has been applied by Street,[7] to explain the dark reverse current $\left(\sim 10^{-12} \mathrm{Amp} / \mathrm{cm}^{2}\right)$ in thin, good-quality a-Si:H photodiodes $(<1 \mu \mathrm{m})$, and it results in a good approximation to the data at low bias. However, for thick detector diodes with high reverse bias, the strong non-uniform electric field in the depleted region enhances the emission of electrons and holes significantly. Therefore a position dependent, field enhanced emission rate should be used. Possible sources of field enhanced emission are; (a) the Poole-Frenkel effect,[8] (b) tunneling [9] and (c) phonon assisted tunneling. [9] These effects are shown schematically in Fig. 4.2.

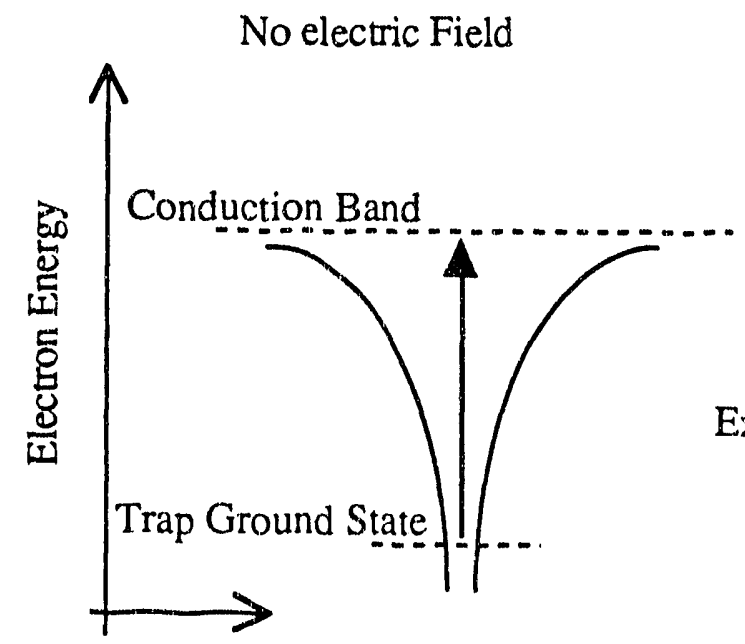

Distance (a)

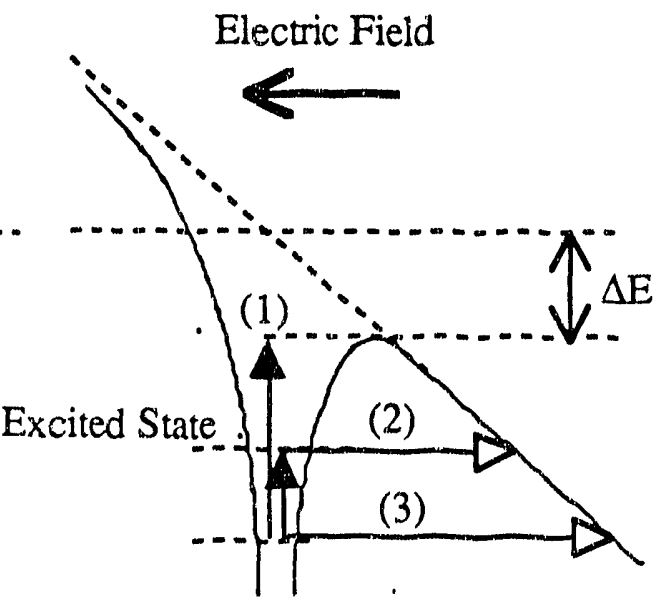

(b)

Fig. 4.2 Mechanisms of electron generation from recombination centers (a) Simple thermal generation, (b) Three effects of strong electric field; (1) Poole-Frenkel barrier lowering, (2) phonon assisted tunneling and (3) direct tunneling. Arrow 4 represents a thermal emission process and $\rightarrow$ represents a field emission process. 
The Poole-Frenkel (P-F) effect, often called the internal Schottky effect, is the fieldenhancement of thermal emission from localized states to the conduction states due to barrier lowering. The emission rate for the case of the 1-d Coulomb potential well becomes

$e_{P F}(x)=\omega_{0} \exp \left[-\left(E_{i}-\Delta E\right) / k T\right]=e_{T H} \exp [\Delta E / k T]$

where $\Delta \mathrm{E}$ is the barrier lowering, [8] and is given by

$$
\Delta \mathrm{E}(\mathrm{x})=\beta_{\mathrm{PF}} \sqrt{\mathrm{F}(\mathrm{x})}
$$

where $F(x)$ is the local electric field and $\beta_{\mathrm{PF}}$ is the Poole-Frenkel constant defined as

$$
\beta_{\mathrm{PF}} \equiv\left(\frac{\mathrm{q}^{3}}{\pi \varepsilon_{\mathrm{o}} \varepsilon_{\mathrm{asi}}}\right)^{1 / 2} \approx 0.022[\mathrm{eV} \sqrt{\mu \mathrm{m} / \mathrm{V}}]
$$

Tunneling from localized states to the conduction band states occurs under a strong electric field. The emission rate has been theoretically calculated by Vincent [10] for the 1-d Coulomb potential well case using the WKB approximation.[11]

$$
\operatorname{erU}(x)=\frac{U}{3 \bar{h}}\left(\frac{U}{E_{i}}\right)^{1 / 2} \exp \left\{-\left(\frac{E_{i}}{U}\right)^{3 / 2}\left[1-\left(\frac{\Delta E}{E_{i}}\right)^{5 / 3}\right]\right\}
$$

where $\mathrm{U}$ is defined as

$$
\mathrm{U} \equiv\left(\frac{3 \mathrm{q} \overline{\mathrm{h}}}{4 \sqrt{2 \mathrm{~m} *}} \times \mathrm{F}(\mathrm{x})\right)^{2 / 3} \approx\left[1.46 \times 10^{-3} \mathrm{~F}[\mathrm{~V} / \mu \mathrm{m}]\right]^{2 / 3} \quad[\mathrm{eV}]
$$

and $\bar{h}$ is Planck constant, $m_{*}$ is the effective electron mass at the trapping center, and $\triangle E$ is the Poole-Frenkel barrier lowering explained previously. The value given is an estimated U for $m_{*}=m_{e}$. For the trap states with a thermal barrier $E_{i} \sim 0.9 \mathrm{eV}$, a comparison of $e_{\mathrm{PF}}$ and $\mathrm{e}_{\mathrm{TU}}$ as a function of electric field $\mathrm{F}$ in Fig. 4.3 shows that $e_{\mathrm{TU}}$ is negligible in the filed range of interest.

Similar arguments show that phonon-assisted tunneling is also negligible. At very low temperatures, however, tunneling may become important. See Appendix $C$ for the numerical expression.

Therefore the bulk generation current is essentially only from Poole-Frenkel emission in the depleted region, or

$$
I_{B G}=q A k T \int_{0}^{d} N_{t}(x) \operatorname{ePF}(x) d x=\frac{I_{T H}}{d} \int_{0}^{d} \exp \left[\left(\beta_{P F} \sqrt{F(x)}\right) / k T\right] d x
$$




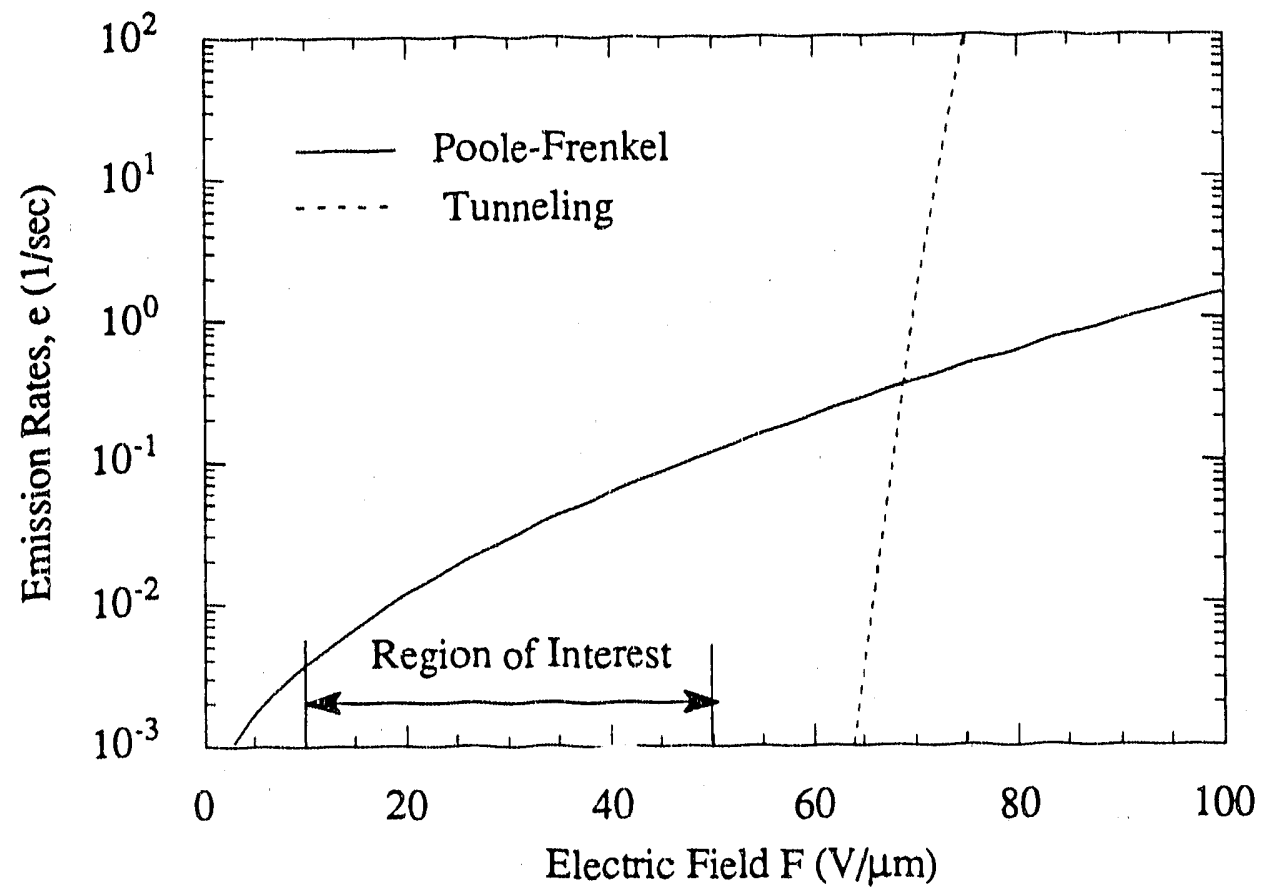

Fig. 4.3 Calculated emission rates of Poole-Frenkel emission and tunneling from a Coulomb potential well with a $0.9 \mathrm{eV}$ barrier height at room temperature.

Using the electric field $F(x)$ calculated in Chapter 3 for both partial and full depletion cases and assuming $N_{t}(x)$ is independent of $x$ in depleted region and zero elsewhere, $\mathrm{I}_{\mathrm{BG}}$ can be expressed as

$$
\begin{aligned}
I_{B G} & \approx \frac{I_{T H}}{d} \frac{2(k T)^{2}}{\beta_{P F}^{2} p} \times\left[\left(\frac{\beta_{P F} \sqrt{F_{0}}-k T}{k T}\right) \exp \left(\frac{\beta_{P F} \sqrt{F_{0}}}{k T}\right)-\left(\frac{\beta_{P F} \sqrt{F_{m}}-k T}{k T}\right) \exp \left(\frac{\beta_{P F} \sqrt{F_{m}}}{k T}\right)\right] \\
& \approx \frac{I_{T H}}{d} \frac{2 k T \sqrt{F_{0}}}{\beta_{P F} p} \exp \left[\left(\beta_{P F} \sqrt{F_{0}}\right) / k T\right]
\end{aligned}
$$

where $F_{0}$ is the peak electric field at the $p-i$ junction $(x=0), F_{m}$ is the minimum electric field and $\rho$ is the slope of the electric field in the deep depletion region given by equation (3.1) in Chapter 3. For the partial depletion case $(Y<1)$ from equation (3.3)

$$
F_{0}=\sqrt{2 \rho V_{a}}, \quad F_{m}=0
$$

and for the full depletion case $(Y>1)$ from equation (3.4)

$$
F_{0}=\frac{2 \rho V_{a}}{d}+\frac{\rho d}{2}, \quad F_{m}=\frac{2 \rho V_{a}}{d}-\frac{\rho d}{2}
$$


(2) Iniection Current:

The injection current is determined primarily by electron injection through the $\mathrm{p}-\mathrm{i}$ interface. Hole injection from the $\mathrm{n}$-layer to the $\mathrm{i}$-layer is negligible both because of the low electric field at the i-n interface and because of the low hole mobility and density.

Doped amorphous silicon has a large number of defect states, almost equal to the active dopant density according to Street's doping model,[12] and the $p$-i interface has a number of gap states equal to or even larger than the p-layer itself. Because of this large density of gap states, electrons in the valence band of the p-layer are injected into the conduction band of the $\mathrm{i}$-layer near the $\mathrm{p}-\mathrm{i}$ interface through multistep hopping processes followed by one of the emission processes described previously. However the barrier height in the p-layer at the p-i interface is $\sim 1.3 \mathrm{eV}$, the energy difference between its Fermi level and the conduction band. Therefore the contribution of barrier injection to the reverse current is negligible compared to that from bulk generation.

At a very high reverse bias, with a $p-i$ interface peak field $F_{0} \geq 50 \mathrm{~V} / \mu \mathrm{m}$, the reverse current and noise increase abruptly. This phenomenon is often called microbreakdown and is reversible. The bias point of micro-breakdown varies from sample to sample and its origin is not clearly understood. It may be attributed to strong injection of electrons from the $\mathrm{p}$-i interface to the $\mathrm{i}$-layer, enhanced by a local high field due to dust particles on the substrate or geometric irregularities at the $\mathrm{p}-\mathrm{i}$ interface. In a-Si:H pin diodes, the operating bias level is usually set below the point where micro-breakdown occurs.

If we neglect the injection current, then the net reverse current is equal to the bulk generation current and it is

$$
I_{R}\left(V_{a}\right)=I_{B G} \approx \frac{I_{T H}}{d} \frac{2 k T \sqrt{F_{0}}}{\beta_{P F} \rho} \exp \left[\left(\beta_{P F} \sqrt{F_{o}}\right) / k T\right]
$$

where $F_{O}$ is given by the equations (4.7) and (4.8) for the partial depletion and the full depletion cases respectively. 


\subsubsection{Noise Generation in a-Si:H pin Diode}

In a reverse-biased a-Si:H pin diode we can expect three types of noise; (1) Shot noise, (2) G-R noise and (3) $1 /$ f noise.

\section{(1) Shot Noise}

Shot noise is always associated with a direct current flow and is present in diodes and bipolar transistors. The passage of carriers across a p-n or Schottky junction is a purely random event and is independent of the number of carriers and the velocity component directed toward the junction. In ideal $\mathrm{p}-\mathrm{n}$ junction diodes or Schottky diodes without generation-recombination, injection is the cnly current source and it is the source of shot noise. The shot-noise-current spectral power $\mathrm{S}_{\text {shot }}$ is white, that is frequency independent, and is given by

$$
S_{\text {shol }}(f)=\frac{\left\langle i_{\text {shol }}^{2}\right\rangle}{\Delta f}=2 q I_{R}
$$

Its derivation is given in Appendix D.

In an a-Si:H diode it is due to the random fluctuation of the injected electrons or holes through the $\mathrm{p}$ - or $\mathrm{n}$ - barrier. Shot noise in reverse biased a-Si:H pin diodes can be calculated without any approximation using the above equation.

\section{(2) Generation-Recombination Noise (G-R Noise)}

When there is a generation-recombination process, for example,

$$
\text { electron }+ \text { empty trap } \leftrightarrow \text { filled trap }
$$

the spectral noise power due to generation-recombination has a Lorentzian shape [13] given by

$$
S_{g r}(f)=\frac{\left.\mathrm{ii}^{2}\right\rangle}{\Delta \mathrm{f}}=4 \overline{\Delta \mathrm{N}_{\mathrm{r}}^{2}} \frac{\tau}{1+\omega^{2} \tau^{2}}
$$


where $\omega$ is $2 \pi f, \Delta N_{r}$ is the fluctuation of the total number of electrons in recombination centers in the device and $\tau$ is the characteristic g- $r$ time constant related to the characteristic frequency $f_{0}=1 /(2 \pi \tau)$. The noise spectrum becomes white at frequencies lower than $f_{0}$ and becomes proportional to $1 / \mathrm{f}^{2}$ at higher frequencies. Typically the characteristic frequency is very low and the trap density can be reduced to a negligible level. As described in the previous section, g-r noise is not significant in c-Si diodes.

In an a-Si:H pin diode under a reverse-bias condition, electrons and holes are generated in the bulk i-layer through recombination centers existing around the quasi Fermi energy level. This process yields generation-recombination noise ( $g-r$ noise) with magnitude proportional to the recombination center density and with a characteristic frequency related to the ernission rate $e_{0}(=1 / \tau)$ by

$$
\mathrm{f}_{\mathrm{o}}=\frac{1}{2 \pi \tau}=\frac{1}{2 \pi} \mathrm{e}_{\mathrm{o}}
$$

For a Fermi energy level $E_{\mathrm{f}}$ located $\sim 0.9 \mathrm{eV}$ below the conduction band mobility edge in the depleted region of intrinsic a-Si:H, the characteristic frequency due to thermal generation is $\sim 10^{-3} \mathrm{~Hz}$ (calculated from the equation (4.2) assuming $\omega_{0}$ to be $10^{12} \mathrm{sec}^{-1}$ ). Even with the generation assisted by the Poole-Frenkel effect, the characteristic frequency will reach only $\sim 1 \mathrm{~Hz}$ for an electric field, F, of $\sim 50 \mathrm{~V} / \mu \mathrm{m}$ (calculated from the equation (4.3)). Therefore the $g-r$ noise is negligible compared to shot noise in the frequency range of interest in the radiation detection applications where a typical pulse shaping time is $\mu$ sec, correspondingly to a frequency $\sim \mathrm{MHz}$.

\section{(3) 1/f Noise}

According to A. van der Ziel,[14] "Flicker noise was discovered by Johnson in vacuum tubes in 1925 and interpreted by Schottky in 1926. ... Later flicker noise was found in a great variety of other components and devices. Because the spectrum varies as l/f $\alpha$, with $\alpha$ close to unity, one often uses the name l/f noise." 
The physical origin of $1 / \mathbb{f}$ noise can be from various sources. In electronic devices, $1 / \mathrm{f}$ noise comes from fluctuations of any electronic quantity having a wide range of characteristic time constants. In c-Si p-n diodes and Schottky diodes, $1 / \mathrm{f}$ (flicker) noise is often observed at very low fivquency, and is attributed to fluctuations of the surface recombination velocity. The $1 / \mathrm{f}$ noise in $\mathrm{c}-\mathrm{Si}$ photodiodes is often reduced using a guard ring structure which reduces the effective recombination surface area of the space charge region.

In general two distinct models have been invoked to explain the origin of $1 / \mathrm{f}$ noise in semiconductors; (a) a mobility fluctuation model [15] and a number fluctuation model [16]

(a) Mobility fluctuation model : Fluctuations of bulk mobility due to lattice scattering have been suggested as a source of $1 / \mathrm{f}$ noise based on Hooge's empirical observations in various resistive devices.[17]

$$
\frac{S_{I}(f)}{I^{2}}=\frac{S_{\mu}(f)}{\mu^{2}}=\alpha_{H} \frac{1}{N f}
$$

where $\mathrm{N}$ is the total number of charge carriers in the device and $\alpha_{H}$ is a proportionality constant called Hooge's constant. It has a value of $\sim 2 \times 10^{-3}$. The observed $\alpha_{H}$ agrees with the above value in resistive devices and long $p-n$ diodes.[18] However for short devices such as FETs and BJTs, $\alpha_{H}$ is orders of magnitude smaller $\left(10^{-6} \sim 10^{-8}\right) .[19 \sim 20]$

(b) Number fluctuation model : This model was originally suggested by McWhorter [16] to explain $1 / \mathrm{f}$ noise observed in MOS structures. According to the model, $1 / \mathrm{f}$ noise originates from trapping and detrapping of charges in the oxide layer near the $\mathrm{Si}_{-} \mathrm{SiO}_{2}$ interface. Depending on the spatial distribution of traps in the oxide, the tunneling mechanism can have a wide range of characteristic time constants. This model can be extended to bulkoriginated $1 / \mathrm{f}$ noise when there are traps having a wide distribution of emission time constants. 
However as pointed by Klaassen,[21] the two models are not contradictory to each other, and total noise may be obtained by adding both together. If the dc current for an ohmic sample is

$$
I=\frac{q \mu N F}{d}
$$

where $\mathrm{N}$ is the total number of cnarge carriers, $d$ is the thickness of the sample, and $F$ is the electric field, then the fluctuation of the current is given by

$$
\Delta \mathrm{I}=\frac{\partial \mathrm{I}}{\partial \mathrm{N}} \Delta \mathrm{N}+\frac{\partial \mathrm{I}}{\partial \mu} \Delta \mu=\frac{\mathrm{I}}{\mathrm{N}} \Delta \mathrm{N}+\frac{\mathrm{I}}{\mu} \Delta \mu
$$

therefore

$$
\frac{S_{I}(f)}{I^{2}}=\frac{S_{N}(f)}{N^{2}}+\frac{S_{\mu}(f)}{\mu^{2}}
$$

Typically one of two sources of fluctuation is dominant in most devices.

The $1 / \mathrm{f}$ noise of a-Si:H devices has been reported in many cases of ohmic $(n-i-n)$ and Schottky diodes. The reported $1 / \mathrm{f}$ noise generally follows a relationship to frequency and dc current given by

$$
S_{V / R}(f)=\frac{\left\langle i_{i j f}^{2}\right\rangle}{\Delta f}=K_{f} \frac{I^{\beta}}{f^{\alpha}}
$$

where $\mathrm{K}_{\mathrm{f}}$ is a proportionality constant and is a function of the device structure and the geometry, $\alpha$ is on the order of 1 , and $\beta$ tends to range between 1 and 2 . Measured values of $\alpha$ and $\beta$ that has been reported are listed in Table 4.1.

The most probable and reasonable origin of $1 / f$ noise in a-Si:H pin diodes is from the transport characteristics of electrons and holes. Because of its intrinsic amorphous structure the large number of defect states in the material can have a wide range of time constants. As explained in Chapter 2, the conduction and valence bands of a-Si:H have broad tails that extend into the gap. Carriers drift under the electric field with mobilities depending on their energy states, so the density and the mobilities are not separable. Therefore fluctuations in mobility and density are also not separable. 
Table 4.1 The coefficient $\alpha$ and $\beta$ for various amorphous silicon devices

\begin{tabular}{|c|c|c|c|}
\hline Sample & Author & $\alpha$ & $\beta$ \\
\hline non-hydrogenated a-Si film & A. D'Amico & $1 \sim 1.2$ & - \\
\hline intrinsic a-Si:H film & F. Z. Bathaei & $0.7 \sim 1.1$ & $\sim 2$ \\
\hline n-type a-Si:H film & C. Parman & $\sim 1$ & $1 \sim 2.5$ \\
\hline p-type a-Si:H film & W. K. Choi & $1-1.5$ & - \\
\hline a-Si:H Schottky (forward) & F. Z. Bathaei & $\sim 1$ & $1 \sim 2$ \\
\hline a-Si:H Schotkky (reverse) & F. Z. Bathaei & - & -0.33 \\
\hline \begin{tabular}{c} 
a-Si:H pin diode (reverse) \\
\hline
\end{tabular}
\end{tabular}

* Data are from references $[22 \sim 27]$.

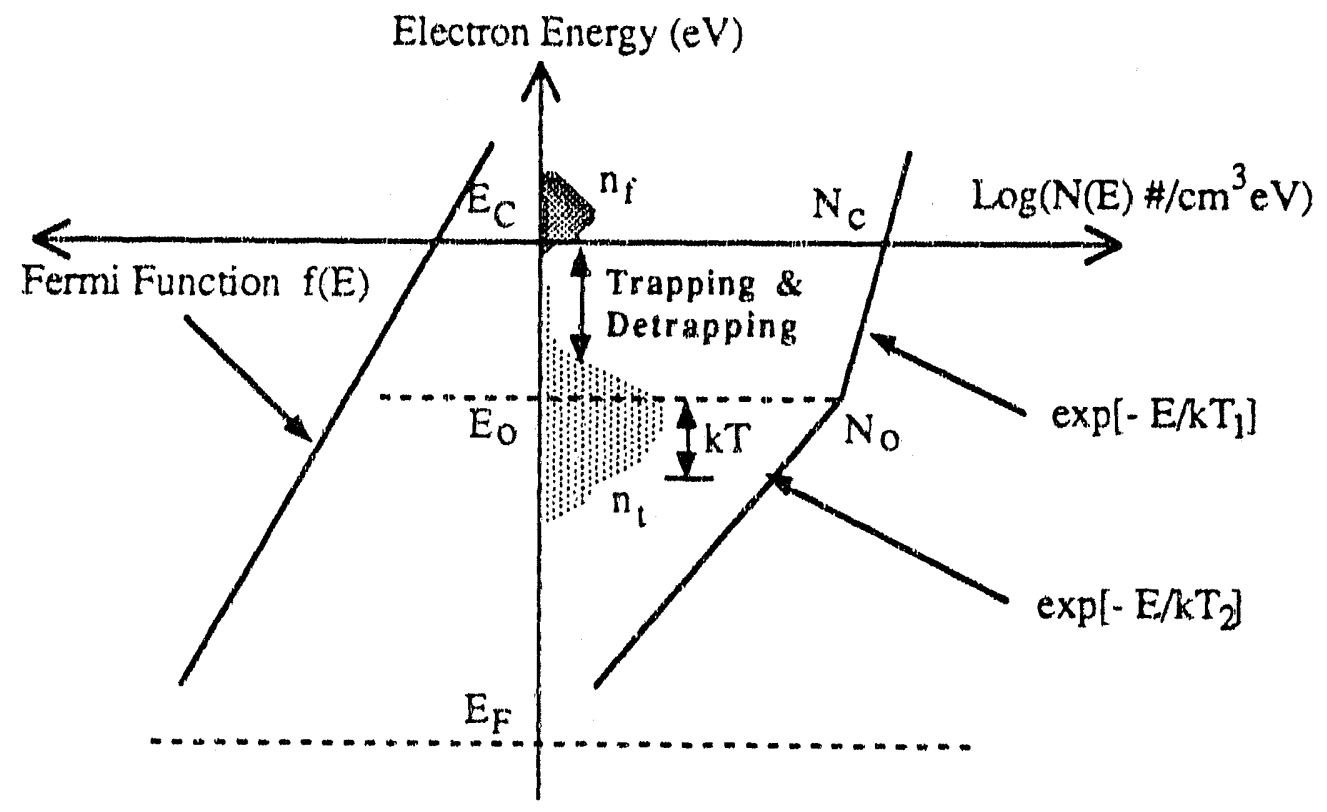

Fig. 4.4 A schematic diagram of the band structure near the conduction band mobility edge of the $i$-layer under reverse bias, showing the noise generation process due to trapping and detrapping of free electrons by shallow band-tail states. 
Also as explained in Chapter 2, at temperature higher than the transition temperature between drift and hopping conduction, the multi-trapping model describes the carrier transport in a way that the electrons having an energy $E$ higher than the mobility edge $E_{c}$ can move freely with the extended drift mobility $\mu_{c}$ and electrons with energy below the mobility edge are localized, i.e. shallow trapped. Then there is a trapping and detrapping equilibrium between the free electrons and trapped electrons as shown in Fig. 4.4. This will cause the fluctuation in reverse current which can yield $1 / \mathrm{f}$ type noise.

From the multitrapping conduction model, we can write

$$
\mu_{e} n_{t o r}=\mu_{c} n_{f}
$$

where $n_{\mathfrak{L t}}$ is the sum of free and shallow trapped electron density, $n_{f}$ and $n_{\mathfrak{l}}$ respectively, and $\mu_{c}$ and $\mu_{e}$ are the extended state mobility and the observed average drift mobility respectively. Then the fluctuation of $\mu_{e}$ is related to the fluctuation of $n_{f}$ by the following relation.

$$
<\Delta \mu_{\mathrm{c}}^{2}>n_{\text {tot }}^{2}=\mu_{\mathrm{c}}^{2}<\Delta \mathrm{n}_{\mathrm{f}}^{2}>
$$

i.e. the mobility fluctuation is equivalent to the number fluctuation of free electrons in a multitrapping conduction model.

A theory based on the number fluctuation model of $1 / f$ noise due to the fluctuations in trapping and detrapping of free carriers to and from the band-tail states has been suggested by Bathaei et al.[23] This theory seems to fit well in the case of ohmic devices where electronic conduction dominates. Here we will apply the number fluctuation model to highly reverse biased thick a-Si:H pin detector diodes with nonuniforn field distribution and Poole-Frenkel generation current model.

Suppose the density of conduction band states consists of two shapes as shown in Fig. 4.4, such as

$$
\begin{array}{ll}
N(E)=N_{1}(E)=N_{0} \exp \left[\left(E-E_{0}\right) / k \Gamma_{1}\right] & E \geq E_{0} \\
N(E)=N_{2}(E)=N_{0} \exp \left[\left(E-E_{0}\right) / k T_{2}\right] & E \leq E_{0}
\end{array}
$$


where $E_{0}$ is $\sim 0.14 \mathrm{eV}$ below $E_{c}$. and $N_{0}$ is the density of states at the knee of the band tail states, i.e., at $E=E_{0} .[28]$ The relation between $N_{0}$ and $N_{c}$ is

$$
N_{0}=N_{c} \exp \left[\left(E_{0}-E_{c}\right) / k T_{1}\right]
$$

where $N_{c}$ is the density of states $\left(-10^{21} \mathrm{~cm}^{-3} \mathrm{eV}^{-1}\right)$ at the conduction band mobility edge. The free electron density $n_{\mathrm{f}}$ in the Boltzmann approximation would then be usually written as

$$
\begin{aligned}
n_{f} & =\int_{E_{c}}^{\infty} N_{1}(E) f(E) d E=N_{0} \int_{E_{c}}^{\infty} \exp \left(\frac{E-E_{0}}{k T_{1}}\right) \exp \left(\frac{E_{f}-E}{k T}\right) d E \\
& =N_{0} \exp \left(\frac{E_{c}-E_{0}}{k T_{1}}\right) \exp \left(\frac{E_{f}-E_{c}}{k T}\right) \times k T \times\left(\frac{T_{1}}{T_{1}-T}\right) \\
& =N_{c} \theta_{1} k T \exp \left(\frac{E_{f}-E_{c}}{k T}\right)
\end{aligned}
$$

where $\theta_{1}=T_{1} /\left(T_{1}-T\right)>1$ and $T_{1}>T$ at room temperature.

The total trapped electrons density $n_{l}$ in the region I of the tail state is

$$
\begin{aligned}
n_{1} & =N_{0} \int_{E_{0}}^{E_{C}} \exp \left[\frac{E-E_{0}}{k T_{1}}\right] \times \exp \left[\frac{E_{f}-E}{k T}\right] d E \\
& =N_{0} \theta_{1} k T \exp \left(\frac{E_{f}-E_{0}}{k T}\right) \times\left[1-\exp \left(\frac{E_{0}-E_{c}}{\theta_{1} k T}\right)\right] \\
& \approx N_{0} \theta_{1} k T \exp \left(\frac{E_{f}-E_{0}}{k T}\right)
\end{aligned}
$$

Similarly for the region II,

$$
\begin{aligned}
n_{2} & =N_{0} \int_{E_{f}}^{E_{0}} \exp \left[\frac{E-E_{0}}{k T_{2}}\right] \times \exp \left[\frac{E_{f}-E}{k T}\right] d E \\
& \approx N_{0} \theta_{2} k T \exp \left(\frac{E_{f}-E_{0}}{k T}\right)
\end{aligned}
$$

where $\theta_{2}=T_{2} /\left(T-T_{2}\right)>1$ and $T_{2}<T$ at room temperature. Therefore the total number of trapped electrons per unit volume is

$$
n_{t}=n_{1}+n_{2}=N_{0}\left(\theta_{1}+\theta_{2}\right) k T \exp \left(\frac{E_{f}-E_{0}}{k T}\right)
$$

A physical picture for the above result is that the trapped electrons are confined within $\theta_{1} \mathrm{kT}$ above the $E_{0}$ level and within $\theta_{2} \mathrm{kT}$ below the $\mathrm{E}_{\mathrm{o}}$ level.

Then the ratio of trapped electrons to free electrons is defined as $R_{e}$ by 


$$
R_{e} \equiv \frac{n_{t}}{n_{f}}=\left(1+\frac{\theta_{2}}{\theta_{1}}\right) \exp \left(\frac{E_{c}-E_{0}}{\theta_{1} k T}\right)
$$

and $R_{e}$ is $5 \sim 10$.

Now let's calculate the fluctuation of free electron density. Since the total electron density ntot at any position is given by

$$
\mathrm{n}_{\mathrm{tot}}=\mathrm{n}_{\mathrm{f}}+\mathrm{n}_{\mathrm{l}}
$$

and is constant in time under steady state, the fluctuation in the number of free electrons is the same as the fluctuation in the number of total trapped electrons. Therefore the spectral power of the fluctuation in the number of free electrons $S_{n f}(f)$ is equivalent to $S_{n_{t}}(f)$.

$$
S_{n_{f}}(f)=S_{n_{l}}(f)
$$

If $n(E) A d x$ is the number of trapped electrons in a volume element $A d x$ within $\Delta E$ at an energy state $E$ which has a certain emission time constant $r$ then the g-r noise theory gives the spectral power of $\Delta n(E)$, similarly to the equation (4.11), such that

$$
\Delta S_{n(E)}(E, f)=4 \overline{\Delta n(E)^{2}} \times \frac{\tau}{1+\omega^{2} \tau^{2}}
$$

where $\overline{\Delta n(E)^{2}}$ is the variance of $n(E)$ averaged in the time domain and is given by

$$
\overline{\Delta n(E)^{2}}=N(E) \times f(E)\{1-f(E)\} A d x \Delta E \approx N(E) \times f(E) A d x \Delta E
$$

where $A$ is the cross-sectional area of the pin diode. If the emission of trapped electrons to the extended state is purely thermionic then from the equation (4.2) the emission time constant $\tau$ is given by the following equation

$$
\tau=\mathrm{e}_{0}^{-1}=\omega_{0}^{-1} \exp \left[\left(E_{c^{-}} \mathrm{E}\right) / \mathrm{kT}\right]
$$

where $\omega_{0}$ is $\sim 10^{12} \mathrm{sec}^{-1}$.

Then the spectral intensity of the fluctuation of the number of total trapped electrons $n_{t}$ is calculated by the integral

$$
S_{n_{t}}(f)=\int_{E_{f}}^{E_{c}} \Delta S_{n(E)}(E, f)=S_{1}(f)+S_{2}(f)
$$


And using the definition of $\tau$ and $\mathrm{dE} / \mathrm{d} \tau=-\mathrm{kT} / \tau . \mathrm{S}_{1}(\mathrm{f})$ is

$$
\begin{aligned}
S_{1}(f) & \equiv 4 \mathrm{Adx} \int_{E_{0}}^{E_{c}} N_{1}(E) f(E) \times \frac{\tau}{1+\omega^{2} \tau^{2}} \mathrm{dE} \\
& =4 \mathrm{Adx} N_{0} \int_{E_{0}}^{E_{c}} \exp \left[\frac{E-E_{0}}{k T_{1}}\right] \times \exp \left[\frac{E_{f}-E}{k T}\right] \times \frac{\tau}{1+\omega^{2} \tau^{2}} \mathrm{dE} \\
& =4 \mathrm{Adx} N_{0} \mathrm{kT} \exp \left[\frac{E_{c}-E_{0}}{\mathrm{kT} T_{1}}\right] \times \exp \left[\frac{E_{f}-E_{c}}{\mathrm{kT}}\right] \int_{\tau_{\mathrm{c}}}^{\tau_{0}} \frac{\left(\omega_{0} \tau\right)^{1 / \theta_{1}}}{1+\omega^{2} \tau^{2}} \mathrm{~d} \tau \\
& =4 \mathrm{Adx} N_{0} \mathrm{kT} \exp \left[\frac{E_{f}-E_{0}}{\mathrm{kT}}\right] \times \exp \left[\frac{E_{0}-E_{c}}{\mathrm{kT} \theta_{1}}\right] \int_{\tau_{c}}^{\tau_{0}} \frac{\left(\omega_{0} \tau\right)^{1 / \theta_{1}}}{1+\omega^{2} \tau^{2}} \mathrm{~d} \tau \\
& =4 \mathrm{Adx} \frac{\mathrm{n}_{1}}{\theta_{1}}\left(\omega_{0} \tau_{0}\right)^{-1 / \theta_{1}} \int_{\tau_{c}}^{\tau_{0}} \frac{\left(\omega_{0} \tau\right)^{1 / \theta_{1}}}{1+\omega^{2} \tau^{2}} \mathrm{~d} \tau \\
& =4 \mathrm{Adx} \frac{\mathrm{n}_{1}}{\theta_{1}} \frac{1}{\left(\tau_{0}\right)^{1 / \theta_{1}}} \int_{\tau_{c}}^{\tau_{0}} \frac{(\tau)^{1 / \theta_{1}}}{1+\omega^{2} \tau^{2}} \mathrm{~d} \tau
\end{aligned}
$$

and if we define $z=\omega \tau=\left(\omega / \omega_{0}\right) \exp \left[\left(E_{c}-E\right) / k T\right]$ then

$$
S_{1}(f)=4 A d x \frac{n_{1}}{\theta_{1}} \frac{1}{\left(\omega \tau_{0}\right)^{1 / \theta_{1}}} \frac{1}{\omega} \int_{z_{c}}^{z_{0}} \frac{(z)^{1 / \theta_{1}}}{1+z^{2}} d z
$$

where $z_{0}=\left(\omega / \omega_{0}\right) \exp \left[\left(E_{c}-E_{0}\right) / k T\right] \approx 2.7 \times 10^{-10} \omega$ and $z_{c}=\left(\omega / \omega_{0}\right) \approx 1 \times 10^{-12} \omega$.

For the frequency range of interest $1<f=\omega / 2 \pi<10^{6} \mathrm{~Hz}, \mathrm{z}_{\mathrm{c}} \ll \mathrm{z}_{0} \ll 1$ therefore the integral becomes approximately

$$
\begin{aligned}
s_{1}(f) & =4 \mathrm{Adx} \frac{\mathrm{n}_{1}}{\theta_{1}} \frac{1}{\left(\omega \tau_{0}\right)^{1 / \theta_{1}}} \frac{1}{\omega} \int_{z_{c}}^{z_{0}}(z)^{1 / \theta_{1}} \mathrm{dz} \\
& =4 \mathrm{Adx} \frac{\mathrm{n}_{1}}{\theta_{1}} \frac{1}{\left(\omega \tau_{0}\right)^{1 / \theta_{1}}} \frac{1}{\omega} \times\left(\frac{1+\theta_{1}}{\theta_{1}}\right)\left[\left(\omega \tau_{0}\right)^{1+1 / \theta_{1}}-\left(\omega \tau_{\mathrm{c}}\right)^{1+1 / \theta_{1}}\right] \\
& =4 \mathrm{Adx} \frac{\mathrm{n}_{1}}{\theta_{1}} \frac{1}{\left(\omega \tau_{0}\right)^{1 / \theta_{1}}} \frac{1}{\omega} \times\left(\frac{1+\theta_{1}}{\theta_{1}}\right)\left(\omega \tau_{0}\right)^{1+1 / \theta_{1}} \\
& \approx 4 \mathrm{Adx} n_{1} \times\left(\frac{1+\theta_{1}}{\theta_{1}^{2}}\right) \tau_{0}
\end{aligned}
$$

i.e. in the frequency range of interest, fluctuation of trapped charges in region 1 gives a white noise or frequency independent noise. This is an expected result because the 
maximum emission time $\tau_{0}\left(E=E_{0}\right)=2.7 \times 10^{-10} \mathrm{sec}$ which corresponds to the characteristic frequency $f_{0}=1 / 2 \pi \tau_{0} \sim 600 \mathrm{MHz}$.

Similarly, for $S_{2}(f)$

$$
\begin{aligned}
& S_{2}(f)=4 A d x N_{0} k T \exp \left[\frac{E_{c}-E_{0}}{k T_{2}}\right] \times \exp \left[\frac{E_{f}-E_{c}}{k T}\right] \int_{\tau_{0}}^{\tau_{f}} \frac{\left(\omega_{o} \tau\right)^{-1 / \theta_{2}}}{1+\omega^{2} \tau^{2}} d \tau \\
& =4 A d x N_{0} k T \exp \left[\frac{E_{f}-E_{0}}{k T}\right] \exp \left[\frac{E_{c}-E_{0}}{k T \theta_{2}}\right] \int_{\tau_{0}}^{\tau_{f}} \frac{\left(\omega_{0} \tau\right)^{-1 / \theta_{2}}}{1+\omega^{2} \tau^{2}} d \tau \\
& =4 \mathrm{Adx} \frac{\mathrm{n}_{2}}{\theta_{2}}\left(\omega_{0} \tau_{0}\right)^{1 / \theta_{2}} \int_{\tau_{0}}^{\tau_{\S}} \frac{\left(\omega_{0} \tau\right)^{-1 / \theta_{2}}}{1+\omega^{2} \tau^{2}} \mathrm{~d} \tau \\
& =4 \mathrm{Adx} \frac{\mathrm{n}_{2}}{\theta_{2}}\left(\tau_{0}\right)^{1 / \theta_{2}} \int_{\tau_{0}}^{\tau_{f}} \frac{(\tau)^{-1 / \theta_{2}}}{1+\omega^{2} \tau^{2}} \mathrm{~d} \tau \\
& =4 A d x \frac{n_{2}}{\theta_{2}}\left(\omega \tau_{0}\right)^{1 / \theta_{2}} \frac{1}{\omega} \int_{z_{0}}^{z_{f}} \frac{1}{\left(1+z^{2}\right)(z)^{1 / \theta_{2}}} d z
\end{aligned}
$$

where $z_{f}=\left(\omega / \omega_{0}\right) \exp \left[\left(E_{c}-E_{f}\right) / k T\right] \approx 4.3 \times 10^{3} \omega$ and $z_{0}=\left(\omega / \omega_{0}\right) \exp \left[\left(E_{c}-E_{0}\right) / k T\right] \approx 2.7$ $x 10^{-10} \omega$. Therefore for the frequency range of interest, the maximum value of the upper limit $z_{f}=4.3 \times 10^{3} \times 2 \pi \times 10^{6} \approx 1.4 \times 10^{10}$ and the minimum value of the lower limit $z_{0}=$ $2.7 \times 10^{-10} \times 2 \pi \times 1=1.7 \times 10^{-9}$.

The integral can be calculated numerically, however, if slope of the deep tail states in region II is very rapid, i.e. $T_{2}<<$ T or $\theta_{2} \gg 1$, the realistic value of $1 / \theta_{2}$ approaches to zero. Therefore the above equation becomes approximately

$$
\begin{aligned}
S_{2}(f) & =4 A d x \frac{n_{2}}{\theta_{2}} \frac{1}{\omega} \int_{z_{0}}^{z_{f}} \frac{1}{\left(1+z^{2}\right)} d z \\
& =4 A d x \frac{n_{2}}{\theta_{2}} \frac{1}{\omega}\left[\tan ^{-1}\left(\omega \tau_{f}\right)-\tan ^{-1}\left(\omega \tau_{0}\right)\right] \\
& =4 A d x \frac{n_{2}}{\theta_{2}} \frac{1}{\omega}\left[\frac{\pi}{2}-0\right] \\
& =A d x n_{f} \frac{1}{\theta_{1}} \exp \left(\frac{E_{c}-E_{0}}{\theta_{1} k T}\right) \times \frac{1}{f} \equiv \frac{A d x n_{f}}{f} Z_{c}
\end{aligned}
$$


using the ratio of $\mathrm{n}_{2}$ to $\mathrm{n}_{\mathrm{f}}$ which is given by

$$
\frac{n_{2}}{n_{f}}=\left(\frac{\theta_{2}}{\theta_{1}}\right) \exp \left(\frac{E_{c}-E_{0}}{\theta_{1} k T}\right)=\theta_{2} Z_{e}
$$

The above equation shows $1 / \mathrm{f}$ dependency in the frequency domain, and the fluctuations of the number of free carriers with the interaction of the deep band-tail states in region II is the source of $1 / \mathrm{f}$ noise in the amorphous silicon diode under reverse bias.

At the limit of $\theta_{2}$ close to a unity then the integral becomes

$$
\begin{aligned}
S_{2}(f) & \equiv 4 \mathrm{Adx} \frac{\mathrm{n}_{2}}{\theta_{2}} \tau_{0} \int_{z_{0}}^{z_{\mathrm{f}}} \frac{1}{\left(1+z^{2}\right) z} \mathrm{dz} \\
& \cong 4 \mathrm{Adx} \frac{\mathrm{n}_{2}}{\theta_{2}} \tau_{0} \times \frac{1}{2} \ln \left(\frac{1+z_{0}^{2}}{1+z_{f}^{2}} \times \frac{z_{f}^{2}}{z_{0}^{2}}\right)
\end{aligned}
$$

If $f$ approaches zero, $S_{2}(f)$ becomes

$$
S_{2}(f)=4 A d x \frac{n_{2}}{\theta_{2}} \tau_{0} \frac{E_{0}-E_{f}}{k T}=\text { const }
$$

and if $f$ approaches to $\infty, S_{2}(f)$ becomes to zero.

Between the two extremes of $\theta_{2}, \infty$ and $1, S_{2}(f)$ has a frequency dependency of a factor $\alpha$ which is a function of $\theta_{2}$ and is normally less than 1 in this model.

$$
S_{2}(f)=A d x \frac{n_{2}}{\theta_{2}} \times \frac{A_{\alpha}}{f^{\alpha}} \quad f_{\min } \leq f \leq f_{\max }
$$

where $A_{\alpha}$ is the normalization constant given by

$$
A_{\alpha}(f) \cong \frac{(1-\alpha) \theta_{2}}{f_{\max }^{1-\alpha}}
$$

which is calculated by

$$
A d x n_{2}=\int_{f_{\min }}^{f_{\max }} S_{2}(f) d f
$$

(a) Case : $\alpha=1$

Now let's calculate the fluctuation in the reverse current using $S_{2}(f)$ in the case of $\alpha$ $=1$ given by the equation (4.15).

Since the electron current is expressed by

$$
I_{e}(x)=q A \mu_{c} F(x) n_{r}(x)
$$

the noise current power due to the fluctuation of the free electron density at position $\mathrm{x}$ is 


$$
\begin{aligned}
\Delta S_{i-e}(x) & =\left(\frac{q \mu_{c} F(x)}{d}\right)^{2} \times S_{2}(x) \\
& =\left(\frac{q \mu_{c} F(x)}{d}\right)^{2} \times \frac{n_{f}(x) A d x}{f} \times Z_{e} \\
& =\frac{q Z_{e} \mu_{c} F(x)}{d^{2} f} \times q \mu_{c} F(x) n_{f}(x) A d x
\end{aligned}
$$

Using the equation (4.17), $\Delta S_{\mathrm{i}-\mathrm{e}}(\mathrm{x})$ becomes

$$
\Delta S_{i \cdot e}(x)=\frac{q}{d^{2} f} \times Z_{e} \mu_{c} F(x) I_{e}(x) d x
$$

Then the resultant noise current power in the external circuit due to fluctuations of total electrons in the i-layer is

$$
S_{\text {I-e }}=\int_{0}^{d} \Delta S_{i-e}(x)=\frac{q}{d^{2} f} \times Z_{e} \mu_{c} \int_{0}^{d} F(x) I_{e}(x) d x
$$

The integral can be calculated analytically using the Poole-Frenkel current equation derived in the previous section given by an equation similar to the equation (4.6),

$$
I_{e}(x)=\frac{I_{T H}}{d} \int_{0}^{x} \exp \left[\left(\beta_{P F} \sqrt{F\left(x^{\prime}\right)}\right) / k T\right] d x^{\prime}
$$

Jinally using an approximation similar to that used in calculating the Poole-Frenkel current,

$$
\begin{aligned}
S_{I-e} & =\frac{q Z_{e} \mu_{c}}{d^{2} f} \times \frac{I T H}{d} \int_{0}^{d} F(x) \int_{0}^{x^{\prime}} \exp \left[\left(\beta_{P F} \sqrt{F\left(x^{\prime}\right)}\right)\right] d x^{\prime} d x \\
& \approx \frac{q Z_{e} \mu_{c}}{d^{2} f} \times \frac{F_{o}^{2}}{2 \rho} \times I_{R}
\end{aligned}
$$

where $\mu_{c}$ can be rewritten with $\mu_{\mathrm{e}}$ by the equation (4.13), which is a measurable quantity from transient photoconductivity or time-of-flight measurement.

$$
\mu_{c}=\mu_{e} \frac{n_{t o t}}{n_{f}}=\mu_{e} \frac{n_{f}+n_{i}}{n_{f}}=\mu_{e}\left(1+R_{e}\right)
$$

where $R_{e}$ is the ratio of the trapped electrons to the free electrons given by the equation (4.14).

By a similar calculation the noise current power due to hole fluctuation is

$$
S_{I-h}=\frac{q}{d^{2} f} \times Z_{h} \mu_{v} \int_{0}^{d} F(x) I_{h}(x) d x
$$

where $\mathrm{Z}_{\mathrm{h}}$ is similarly defined for holes, and $\mu_{\mathrm{V}}$ is the extended state hole mobility. 
Since the hole current density in depleted region $\mathrm{I}_{\mathrm{h}}(\mathrm{x})$, can be rewritten by

$$
I_{h}(x)=I_{R}-I_{e}(x)
$$

SI-h becomes

$$
\begin{aligned}
S_{I-h} & =\frac{q Z_{h} \mu_{v}}{d^{2} f} \times\left[I_{R} \int_{0}^{d} F(x) d x-\int_{0}^{d} F(x) I_{e}(x) d x\right] \\
& =\frac{q Z_{h} \mu_{v}}{d^{2} f} \times I_{R} \times\left(V_{a}-\frac{F_{0}^{2}}{2 p}\right)
\end{aligned}
$$

Finally the total noise current power of the $1 / \mathrm{f}$ noise component in an a-Si:H pin diode is

$$
S_{1 / f}=S_{I-e}+S_{I-h}=\frac{q I_{R}}{d^{2} f} \times\left[Z_{h} \mu_{v} V_{a}+\left(Z_{e} \mu_{c}-Z_{h} \mu_{v}\right) \frac{F_{Q}^{2}}{2 \rho}\right]
$$

(b) Case : $\alpha \leq 1$

For the general case of $\alpha \leq 1$, we must muitiply $A_{\alpha}$ and replace $f$ with $f \alpha$. Then it can be written in a form given by the equation (4.12)

$$
S_{1 / \mathbb{R}}(f)=\frac{\left\langle i_{1 / p}^{2}\right\rangle}{\Delta f}=K_{f} \frac{I_{R}^{\beta}}{f^{\alpha \alpha}}
$$

where $K_{f}, \alpha$ and $\beta$ can be numerically determined.

Then the total noise current power $S_{\text {det }}(f)$ of a reverse-biased a-Si:H pin diode is a sum of $S_{\text {shot }}(f)$ and $S_{1 /(}(f)$, from the above equation and the equation (4.10),

$$
S_{\text {det }}(f)=\frac{\leq i^{2}>}{\Delta f}=S_{\text {shot }}(f)+S_{1 / f}(f)=2 q I_{R}+K_{f} \frac{I_{R}^{\beta}}{f^{\alpha}}
$$




\subsection{Noise of Thin-Film-Transistor Amplifiers}

In a proposed a-Si:H pixel radiation detector-amplifier system, the front-end stage of signal processing electronics are made of a-Si:H or poly-Si TFTs. Since the front-end TFT of the preamplifier system has the dominant contribution to the noise of the total system noise, noise characteristics of individual TFTs with other basic characteristics are measured and analyzed in this section.

\subsection{1 a-Si:H and poly-Si TFTs}

Hydrogenated amorphous and poly-silicon thin-film-transistors (TFTs) are at present widely used as a read-out circuit for linear image sensors [29] and as driving circuits of liquid crystal display devices where TFTs operate as switching elements. An operational-amplifier has been made out of poly-Si TFTs by Lewis,[30] however, the linear properties of both a-Si:H and poly-Si TFTs are yet in question for realistic applications. In this section we will show some basic properties as well as noise characteristics of a-Si:H and poly-Si TFTs currently available from industry and discuss the application of these TFTs in large-area position sensitive a-Si:H radiation detectors.

(1) a-Si:H TFTs : A-Si:H TFTs are normally composed of a thin $(\sim 0.5 \mu \mathrm{m})$ intrinsic aSi:H layer as a main current channel and amorphous silicon nitride of $0.3 \mu \mathrm{m}$ as a gate insulator. Some other materials such as silicon oxide and tantalum oxide [31] are used as gate insulators, however, their characteristics are not reliable. Amorphous silicon TFTs are the staggered-inverted type as shown in Fig. 4.5(a), and channels are defined by a selfaligning technique using gate metal as a mask for photo-etching by shining light through the glass substrate. Because of the low hole mobility, only n-channel a-Si:H TFTs are practically applicable and they are operated in the accumulation mode which means the 
electrons are accumulated at the interface of the gate insulator and the intrinsic a-Si:H layer (slightly n-type) under the positively biased gate.

(2) Poly-Si TFTs : Poly-Si TFTs are becoming very important elements in making driver circuits for large-area image devices such as LCD screens [32] and 2-D scanners because of their capability for fast speed and reliable operation. Both n-channel and p-channel TFTs can be made by ion-implantation doping followed by a high temperature annealing. In general two methods of making a poly-Si film out of an amorphous silicon film on a substrate are used; (1) post-annealing in the temperature range of $600^{\circ} \sim 900^{\circ} \mathrm{C}$ [33] and (2) laser-induced recrystallization.[34] The second method, with careful treatment, may produce larger crystalline grain but the first method is widely used because of low cost and reliability in processing. Annealing at $900^{\circ} \mathrm{C}$ requires a quartz substrate because of the high temperature. The sample TFTs described here were made by the annealing technique at Xerox and GSI and its schematic diagram is shown in Fig. 4.5 (b).

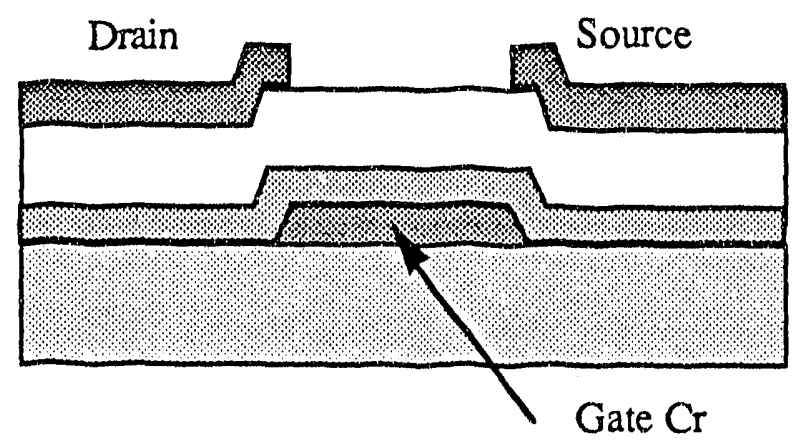

$\mathrm{Cr}$ a-Si:H $(500 \mathrm{~nm})$ $\mathrm{Si}_{3} \mathrm{~N}_{4}(300 \mathrm{~nm})$

Substrate

(a)

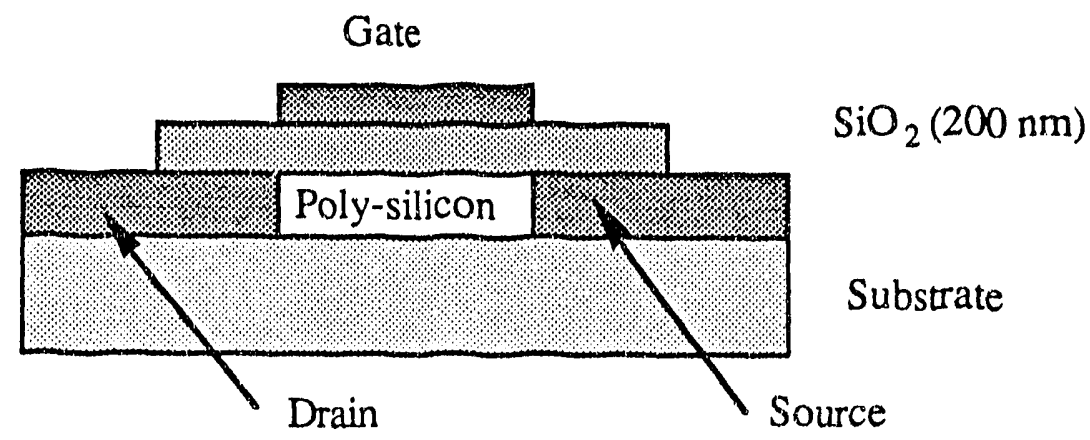

(b)

Fig.4.5 Schematic cross-sectional views of (a) a-Si:H and (b) poly-Si TFTs. 


\subsubsection{Noise Sources in TFT's}

In MISFET (metal-insulator FET), basically two types of noise components are present; (1) thermal noise from the finite channel-conductance and (2) $1 / \mathrm{f}$ noise from the interaction of channel electrons with the traps located in the gate insulator near the siliconinsulator interface. In thin-film-transistors, we can expect that the trapping and detrapping at the band tail states in bulk amorphous silicon and the generation-recombination at the crystalline grain boundaries in poly-silicon produce an additional $1 / \mathrm{f}$ noise component.

\section{(1) Thermal Nyquist) noise :}

When the TFTs are in the operation region, i.e., the gate bias is in the saturation region, the drain-to-source current $I_{d s}$ has a functional dependence on the gaie bias $V_{g s}$ but is independent of the drain-to-source bias $V_{d s}$.

$$
I_{\mathrm{ds}}=\frac{1}{2} \mu_{\mathrm{fe}} C_{\mathrm{o}}\left(\frac{\mathrm{W}}{\mathrm{L}}\right) \times\left(\mathrm{V}_{\mathrm{gs}}-\mathrm{V}_{\mathrm{T}}\right)^{2}
$$

where $\mu_{\mathrm{fe}}$ is the field effect mobility, $\mathrm{W}$ and $\mathrm{L}$ are the channel width and channel length respectively, $V_{g s}$ and $V_{T}$ are respectively the gate bias and threshold voltage, and $C_{o}$ is the capacitance per unit area of the gate insulator $=\varepsilon_{0} \varepsilon_{j} / t_{i}$ where $\varepsilon_{0}$ and $\varepsilon_{\mathrm{i}}$ are the dielectric constant of the vacuum and the relative dielectric constant of the gate insulator and $t_{i}$ is the thickness of the insulator. The transconductance in the saturation region is given by

$$
g_{m} \equiv \frac{d I_{d s}}{d V_{g s}}=\mu_{f e} C_{0}\left(\frac{W}{L}\right) \times\left(V_{g s}-V_{T}\right)
$$

Noise in FETs appear as a fluctuation of the drain current $I_{d s}$ and the thermal noise due to channel conductarice is given by the following equation [35]

$$
\frac{\left\langle\mathrm{i}_{\mathrm{h}}^{2}\right\rangle}{\Delta \mathrm{f}} \approx 4 \mathrm{kT} \theta \mathrm{g}_{\mathrm{m}}
$$

where $\theta$ is a process dependent constant and is normally $\sim 2 / 3$. The input-referred thermal noise power $S_{\mathrm{th}}(\mathrm{f})$ is obtained by dividing the output drain curtent noise by the square of $g_{m}$.

$$
S_{\mathrm{th}}(\mathrm{f})=\frac{\left\langle v_{\mathrm{lh}}^{2}\right\rangle}{\Delta \mathrm{f}}=4 \mathrm{kT} \times \frac{\theta}{\mathrm{g}_{m}}
$$


(2) 1/f noise :

$1 / \mathrm{f}$ noise in MISFET originates from the fluctuation of charge carriers at the interface between gate insulator and semiconductor. Therefore it is depending on the details of the fabrication process. Empirically, input referred $1 / \mathrm{f}$ noise power in FETs is described by a similar equation to the equation (4.12)

$$
S_{\mathrm{If}}(f)=\frac{\left\langle v_{1 / \mathrm{f}}^{2}\right\rangle}{\Delta \mathrm{f}}=K_{\mathrm{l}} \times \frac{I_{d s}^{\beta}}{\mathrm{f}^{\alpha}}
$$

where $\alpha \sim 1$ and $\beta$ is nearly zero for crystalline silicon MCSFETs. Therefore noise power is weakly dependent on the drain current.[36] The constant $K_{t}$ depends on the device geometry and the process and is normally inversely proportional to the channel capacitance $=C_{0} W L$.

Since amorphous silicon and poly-silicon TFTs have a similar structure to the crystalline MOSFET, we can expect a similar functional dependence on the drain current and frequency. However, because the semiconductor layer is made by deposition, more defects and corresponding trap states at the interface and in the bulk exist than the crystalline Si MOSFET. From the equations (4.21) and (4.22), the total input referred noise of a-Si:H and poly-Si TFTs can be expressed as

$$
S_{T F T}(f)=\frac{\left\langle V_{T F T}^{2}\right\rangle}{\Delta f}=\frac{4 k T \theta}{g m}+\frac{K_{l}}{C_{o} W L} \times \frac{I_{d s}^{\beta}}{f^{\alpha}}
$$

where $\theta, \alpha, \beta$ and $K_{\mathrm{l}}$ can be found experimentally.

\subsection{Optimization of Total System Noise by Pulse Shaping}

The output signal from the detector or from the preamplifier is normally shaped into a short pulse form to avoid pulse pile-up and to make further signal processing easier, for example, analog-to-digital conversion, memory, display etc. The pulse shaping amplifier, however, also acts as a band pass filter to reduce systern noise, and achieve an optimum 
signal-to-noise ratio. Optimization of the signal-to-noise ratio requires a formulation of the total system noise, a sum in quadrature of the detector noise and the amplifier noise, as a function of design parameters, such as capacitance and operation bias level of the detector and amplifier, and the shaping time of the shaping amplifier, etc. Depending on specific purposes, there are many types of shaping amplifiers. However, we will discuss two simple cases; (a) CR-(RC) filter as an example of time-invariant filters and (b) a gated integrator shaper as an example of time-variant filters.

\subsubsection{Conversion of Noise Power Spectra to Equivalent Noise Charge}

As mentioned in the introduction of this chapter, noise of a radiation detection system is generally expressed by the equivalent noise charge (ENC) in the measurementtime domain rather than the noise power spectra (NPS) in the frequency domain. Hence it is necessary to relate the noise power spectra to the equivalent noise charge. This conversion from the frequency domain to the tirne domain is physically accomplished in a shaping amplifier, which basically is a filter network with a characteristic time constant, called a shaping time or a peaking time. Mathematically the conversion is a convolution of the noise spectrum with a transfer function of the shaping amplifier, and is given by

$$
(E N C)_{j}^{2} \equiv N_{j}^{2}=\frac{1}{A_{s}^{2}} \times \int_{0}^{\infty} S_{j} \times G^{2} d f
$$

where $A_{S}$ is a normalization constant or gain of the shaping amplifier to the unit charge, $G$ is the transfer function of the shaping amplifier and $S_{j}$ is the frequency spectrum of the noise power component $\mathrm{j}$.

The noise components from the detector and from the front-end amplifier can be classified into two different types as shown in the Fig. 4.6; (a) a parallel noise-current source, and (b) a series noise-voltage source, $\left\langle v^{2}\right\rangle$. (a) Parallel noise sources (detector shot noise, detector $1 / f$ noise) are represented as noise current sources, $\left\langle i^{2}\right\rangle$ and (b) series 


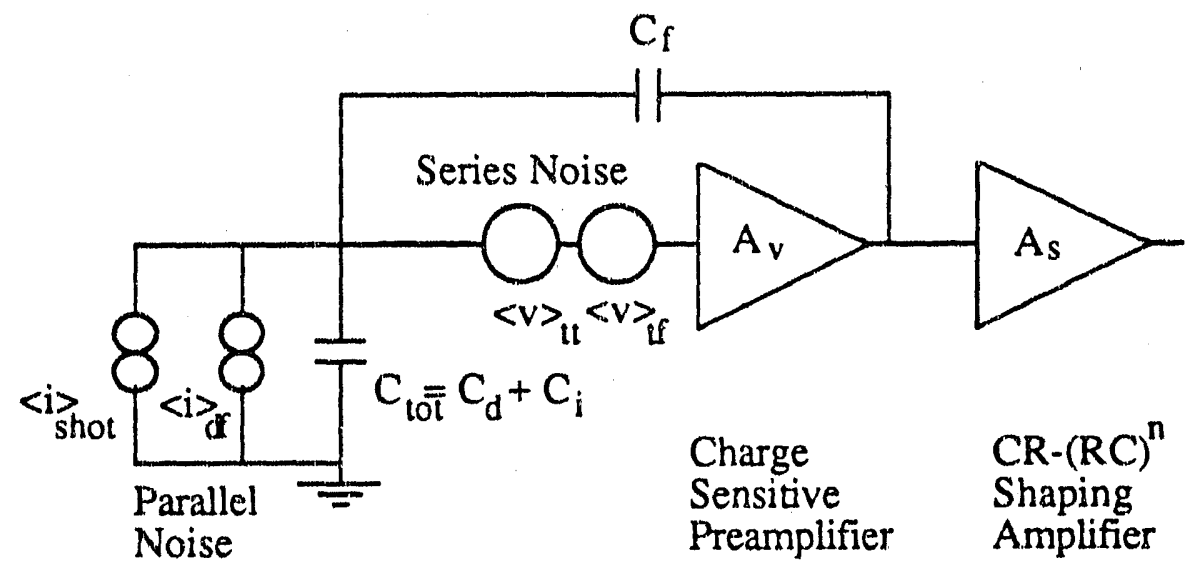

Fig. 4.6 Parallel and series noise sources in detector-amplifier system. (shot $=$ shot noise, $\mathrm{df}=$ detector $1 / \mathrm{f}$ noise, $t \mathrm{t}=$ transistor thermal noise and $\mathrm{tf}=$ transistor $1 / \mathrm{f}$ noise).

noise sources are noise voltage sources (thermal noise and $1 / \mathrm{f}$ noise of the front end TFT in the preamplifier). Also any series resistance component between the detector and the preamplifier introduces additional thermal noise which can be expressed by

$$
S_{S T}(f)=\frac{\left.s v_{S P}^{2}\right\rangle}{\Delta f}=4 k T R_{s}
$$

where $R$ is the magnitude of the series resistance.

The equivalent noise charges from these sources can be obtained using either one of the following equations,

$$
\begin{aligned}
& N_{i}^{2}=\frac{1}{A_{S}^{2}} \times \frac{1}{q^{2}} \int_{0}^{\infty} \frac{\left\langle i^{2}\right\rangle}{(2 \pi f)^{2}} \times G^{2} d f \\
& N_{v}^{2}=\frac{1}{A_{S}^{2}} \times \frac{C_{10 t}^{2}}{q^{2}} \int_{0}^{\infty}\left\langle v^{2}\right\rangle \times G^{2} d f
\end{aligned}
$$

where $q$ is an electronic charge and $C_{10 t}$ is the sum of detector capacitance $C_{d}$ and input capacitance $\mathrm{C}_{\mathrm{i}}$ of the preamplifier (the gate-to-source overlapping capacitance of the frontend TFT $C_{i} \approx C_{0} W L$ where $L$ is the channel length). A parallel noise-current source, $\left\langle\mathrm{i}^{2}\right\rangle$ is converted into an equivalent series noise-voltage source, $\left\langle v^{2}\right\rangle$ by the following relation

$$
\left.\left\langle v^{2}\right\rangle=\frac{1}{\left(\omega C_{101}\right)^{2}} \times<i^{2}\right\rangle
$$


Then the total system noise power spectrum $S_{\text {sys }}$ is obtained by equations (4.19), (4.23) and (4.24) and is

$$
\begin{aligned}
& S_{\text {sys }} \wedge f=\left\langle v_{\text {st }}^{2}\right\rangle+\left\langle v_{\mathrm{tl}}^{2}\right\rangle+\left\langle v_{\mathrm{tf}}^{2}\right\rangle+\frac{\left\langle\mathrm{i}_{\text {shot }}^{2}\right\rangle}{\left(\omega \mathrm{C}_{\mathrm{tot}}\right)^{2}}+\frac{\left\langle\mathrm{i}_{\mathrm{l} / \mathrm{f}}^{2}\right\rangle}{\left(\omega \mathrm{C}_{\mathrm{tot}}\right)^{2}} \\
& =4 k T R_{s}+\frac{4 k T \theta}{g_{m}}+\frac{2 \pi K_{t} I_{d s}^{\beta_{s}}}{C_{o} W L} \frac{1}{\omega}+\frac{2 q I_{R}}{\left(C_{t o t}\right)^{2}} \frac{1}{\omega^{2}}+\frac{2 \pi K_{f} l_{R}^{\beta_{d}}}{\left(C_{t o t}\right)^{2}} \frac{1}{\omega^{3}} \\
& =K_{0}+\frac{K_{1}}{\omega}+\frac{K_{2}}{\omega^{2}}+\frac{K_{3}}{\omega^{3}}=\sum_{m=0}^{3} \frac{K_{m}}{\omega^{m}}
\end{aligned}
$$

\subsubsection{CR-(RC) $)^{\mathrm{n}}$ Pulse Shaping}

When a CR-(RC) ${ }^{n}$ filter is used as a shaping amplifier with a shaping time $\tau$ which is equal to the $\mathrm{RC}$ time constant, the transfer function is given by

$$
G_{n}^{2}(\omega)=\frac{a^{2 n} \omega^{2}}{\left(a^{2}+\omega^{2}\right)^{n+1}}
$$

where $\omega$ is $2 \pi \mathrm{f}, a=1 / \mathrm{RC}=\mathrm{n} / \tau$. $\tau$ is the shaping time or the peaking time because the output signal pulse of a CR- $(R C)^{n}$ filter from a step input at $t=0$, rise to a peak value at $t=$ $\tau$. The gain of a $C R-(R C)^{n}$ shaping amplifier $A_{S}$ is given by

$$
A_{s}=\frac{n^{n}}{n ! e^{n}}
$$

where $\mathrm{e}$ is the natural logarithm base.

Using the total system noise expression in the frequency domain, $S_{S y s}$, the equivalent noise charges of the detector and amplifier noise components are obtained for $n$ $=1$ and $n=4$ (Gaussian shaping) and shown in Table 4.2. For more details see Appendix E. In nuclear electronics, detector shot noise and the amplifier thermal noise are often called step noise and delta noise respeciavely.

Fig. 4.7 is a schematic of these noise sources as a function of shaping time. Their magnitudes are not scaled in the figure but can be evaluated from the equations in the above table with various design and operation parameters. 
Table 4.2 Equivalent noise charges (electrons in rms) from detector-amplifier system

\begin{tabular}{|c|c|c|c|}
\hline \multirow[t]{2}{*}{ Device } & \multirow[t]{2}{*}{ Noise Type } & \multicolumn{2}{|c|}{ Pulse Shaping Amplifier Type } \\
\hline & & CR-RC & $\mathrm{CR}-(\mathrm{RC})^{4}$ \\
\hline \multirow{3}{*}{ Detector } & Shot Noise & $1.9 \frac{\mathrm{IR}}{\mathrm{q}} \tau$ & $0.9 \frac{\mathrm{I} R}{\mathrm{q}} \tau$ \\
\hline & 1/f Noise* & $7.4 \frac{\mathrm{K}_{\mathrm{f}} \mathrm{I}_{\mathrm{R}}^{\beta_{\mathrm{d}}}}{\mathrm{q}^{2}} \ln \left(1 / \tau \omega_{\min }\right) \tau^{2}$ & $1.6 \frac{\mathrm{K}_{\mathrm{f}} \mathrm{I}_{\mathrm{R}}^{\beta_{\mathrm{d}}}}{\mathrm{q}^{2}} \ln \left(1 / \tau \omega_{\min }\right) \tau^{2}$ \\
\hline & Contact Noise & $0.92 \frac{C_{\mathrm{tol}^{2}} 4 \mathrm{kT} \mathrm{R}}{\mathrm{q}^{2}} \frac{1}{\tau}$ & $1.0 \frac{\mathrm{C}_{\mathrm{lot}}^{2} 4 \mathrm{kT} \mathrm{R}}{\mathrm{q}^{2}} \frac{1}{\tau}$ \\
\hline \multirow[t]{2}{*}{ Amplifier } & Thermal Noise & $0.92 \frac{\mathrm{C}_{10 \mathrm{r}^{2}} 4 \mathrm{kT} \theta}{\mathrm{g}^{2} \mathrm{~g}_{\mathrm{m}}} \frac{1}{\tau}$ & $1.0 \frac{\mathrm{C}_{\mathrm{tot}}{ }^{2} 4 \mathrm{kT} \theta}{\mathrm{q}^{2} \mathrm{gm}} \frac{1}{\tau}$ \\
\hline & 1/f Noise & $3.7 \frac{\mathrm{C}_{\mathrm{tot}}^{2} \mathrm{~K}_{\mathrm{t}} \mathrm{I}_{\mathrm{ds}}^{\beta_{\mathrm{s}}}}{\mathrm{q}^{2} \mathrm{C}_{\mathrm{o}} \mathrm{WL}}$ & $3.3 \frac{\mathrm{C}_{\mathrm{tot}}^{2} \mathrm{~K}_{\mathrm{t}} \mathrm{I}_{\mathrm{ds}}^{\mathrm{B}_{\mathrm{t}}}}{\mathrm{q}^{2} \mathrm{C}_{0} \mathrm{WL}}$ \\
\hline
\end{tabular}

* where $\omega_{\min }$ is determined by $\omega_{0} \exp \left[\left(E_{\min }-E_{c}\right) / k T\right]$.

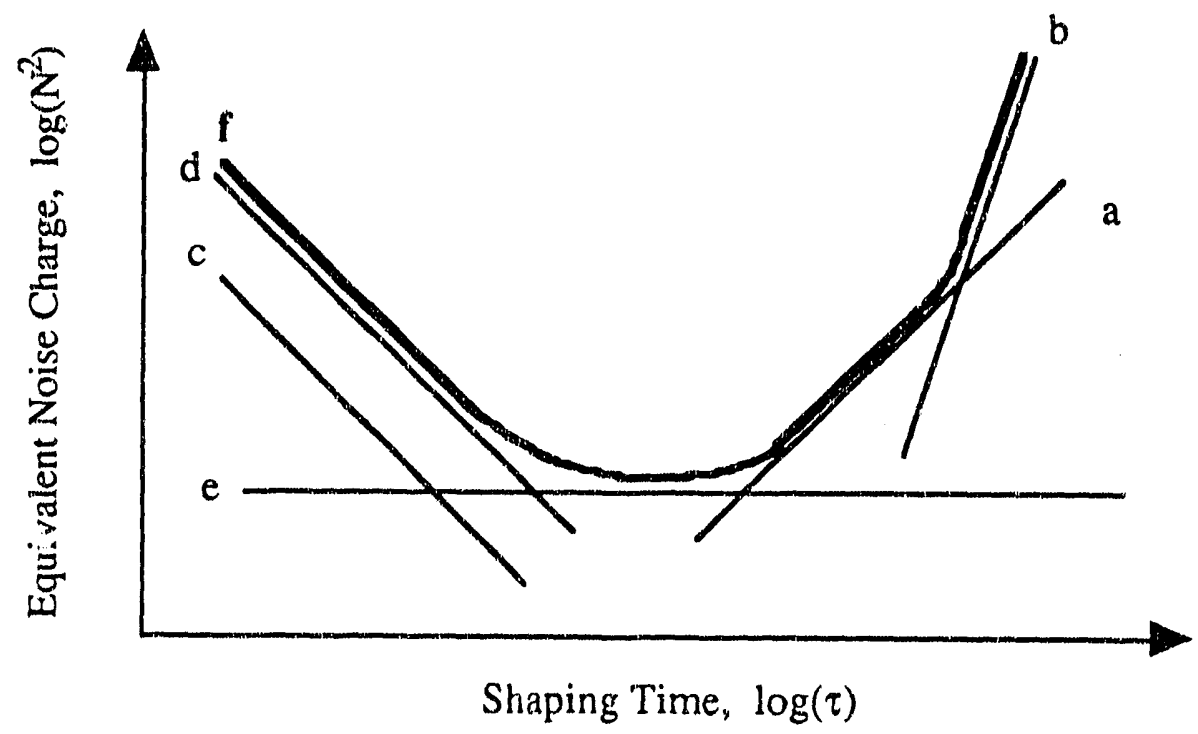

Fig. 4.7 Schematic representation of equivalent noise charges from various sources as a function of the shaping time; (a) detector shot noise $\sim \tau$, (b) detector $1 / \mathrm{f}$ noise $~$ $\tau^{2}$, (c) series contact noise $\sim \tau^{-1}$, (d) amplifier thermal noise $\sim \tau^{-1}$, (e) amplifier $1 / f$ noise, independent of $\tau$ and $(f)$ total system noise. 


\subsubsection{Time-Variant Filter}

In this section, we will briefly review a gated-integrator filter as an example of an active time-variant filter and discuss a correlated double sampling scheme to optimize the noise contribution from the front-end amplifier, i.e, thermal noise and $1 / \mathrm{f}$ noise of TFTs.

\section{(1) Gated-Integrator Filter}

A gated-integrator filter is the most common type of active filter.[37] Fig. 4.8 is a schematic of a gated integrator. At the start of the detector signal, switch 1 is closed and switch 2 is open. Then the signal current is integrated until switch 1 is opened and the signal output keeps its height for readout of the signal output by the following stage of the system until switch 2 is closed. This type of operation is equivalent to the function of RC integrator or low-pass filter to the noise current. It has a trapezoidal weighting function to the noise in the time domain. A time domain analysis of this filter is detailed in Appendix $F$.

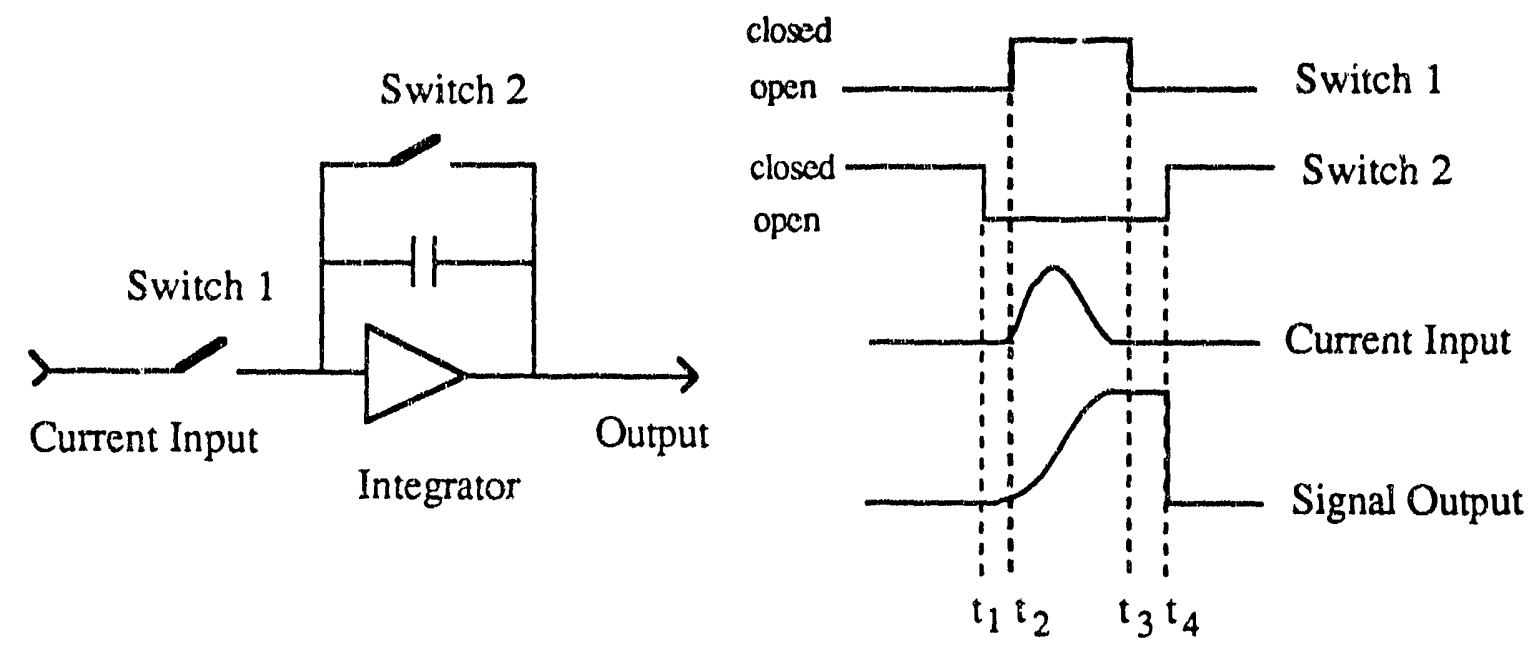

Fig. 4.8 A schematic diagram of an gated-integrator and the time-variant pulse shaping. 
Advantages of time variant filters with a trapezoidal weighting function are that (a) precise timing is not required because of the existence of the flat top region, (b) pulse pileup is considerably reduced due to the rapid termination of the output pulse and (c) noise incurred in one measurement does not affect to the next measurement following the end of the first measurement interval.

\section{(2) Correlated Double Sampling}

Fig. 4.9 shows a correlated double sampling scheme coupled to a gated-integrator and a timing diagram of the correlated double sampling. This scheme has been widely used in CCDs to reduce the switching transients and to eliminated the reset noise of the integrator switch [38] because they are fully correlated between the clamp and sample circuit. During the first sampling time $T_{i 1}$ prior to signal readout, the holding capacitor $C_{h}$ accumulates charges flowing from a reference voltage $V_{\text {clamp }}$ with noise of the preamplifier. In $\Delta T$ after the first sampling time, the input signal charge from detector through the preamplifier circuit with all noise is integrated during the second sampling time $T_{i 2}$ and is compared to the stored charge in $\mathrm{C}_{\mathrm{h}}$. The output is proportional to the signal charge subtracted from the reference charge.

The effect on the white noise of the preamplifier is to increase it at the CDS output due to aliasing.[39] However, it can reduce the low frequency $1 / \mathrm{f}$ noise of the preamplifier.[40] Therefore there is a big advantage to this scheme for a- $\mathrm{Si}: \mathrm{H}$ or poly-Si TFT preamplifiers which have large $1 / \mathrm{f}$ noise components compared to the white thermal noise from the channel resistance. 

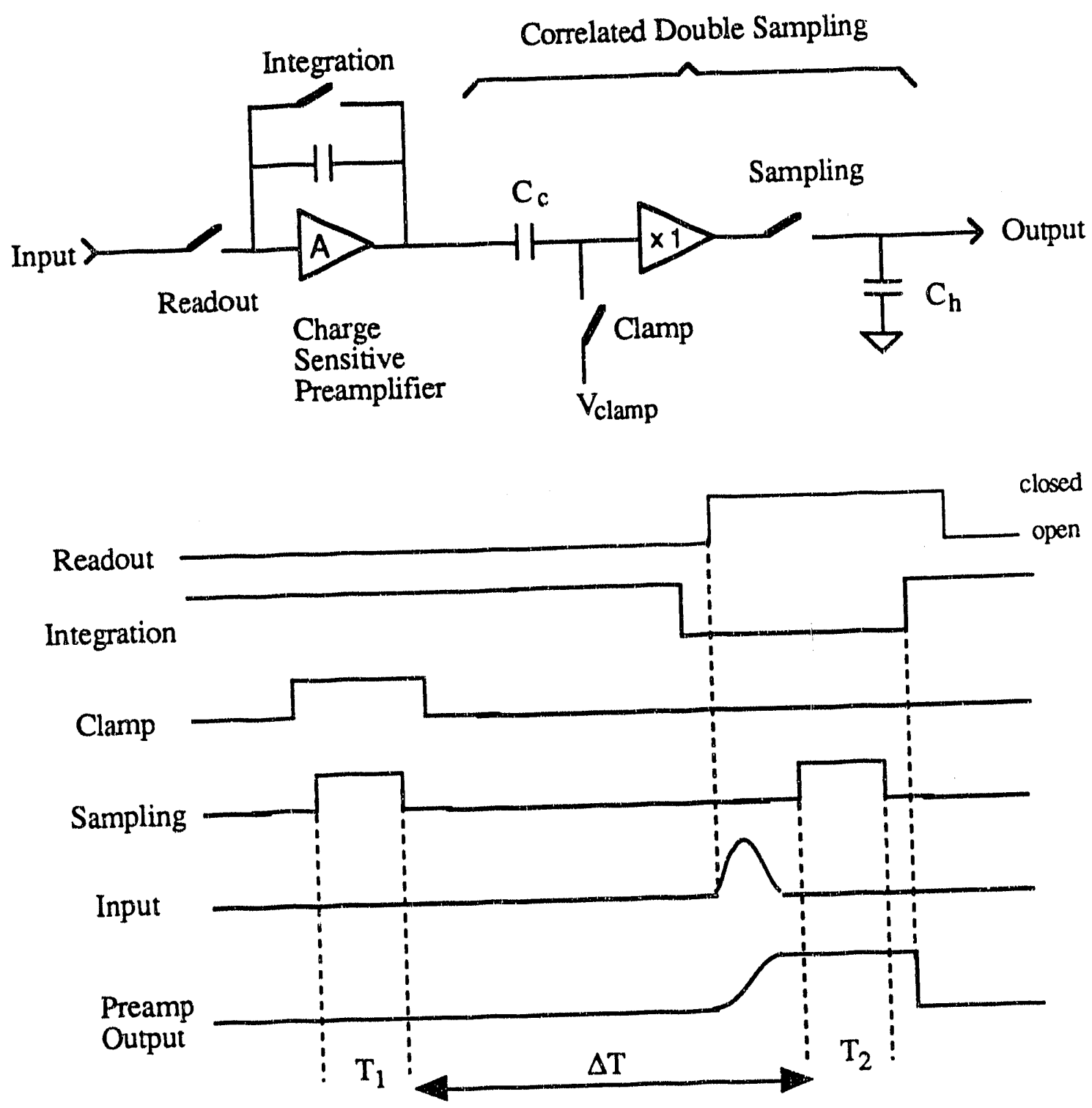

Fig. 4.9 A schematic diagram of the correlated double sampling scheme. 


\subsection{Measurements and Calculation Results}

\subsubsection{Current and Noise of a-Si:H Detectors}

Since the diodes are reverse biased when used as radiation detectors, the dark reverse current and its noise power spectra are measured under reverse biased condition. Fig. 4.10 shows the measurement set-up used. While the ammeter measures the reverse current as a function of the applied bias voltage, the frequency spectra of the output noise power from a charge-sensitive amplifier is amplified and sampled through the setup (A) in the figure. An aliasing effect due to digital sampling is removed by a set of butterworth filters for a measurement frequency range set and changed at the oscilloscope. The noise data were recorded and their Fourier transforms were calculated using fast Fourier transforms in a PC. The sample diodes measured in this experiment had thickness ranging from 5 to $45 \mu \mathrm{m}$, from three sources, Xerox PARC, Glasstech Solar Inc and LBL.

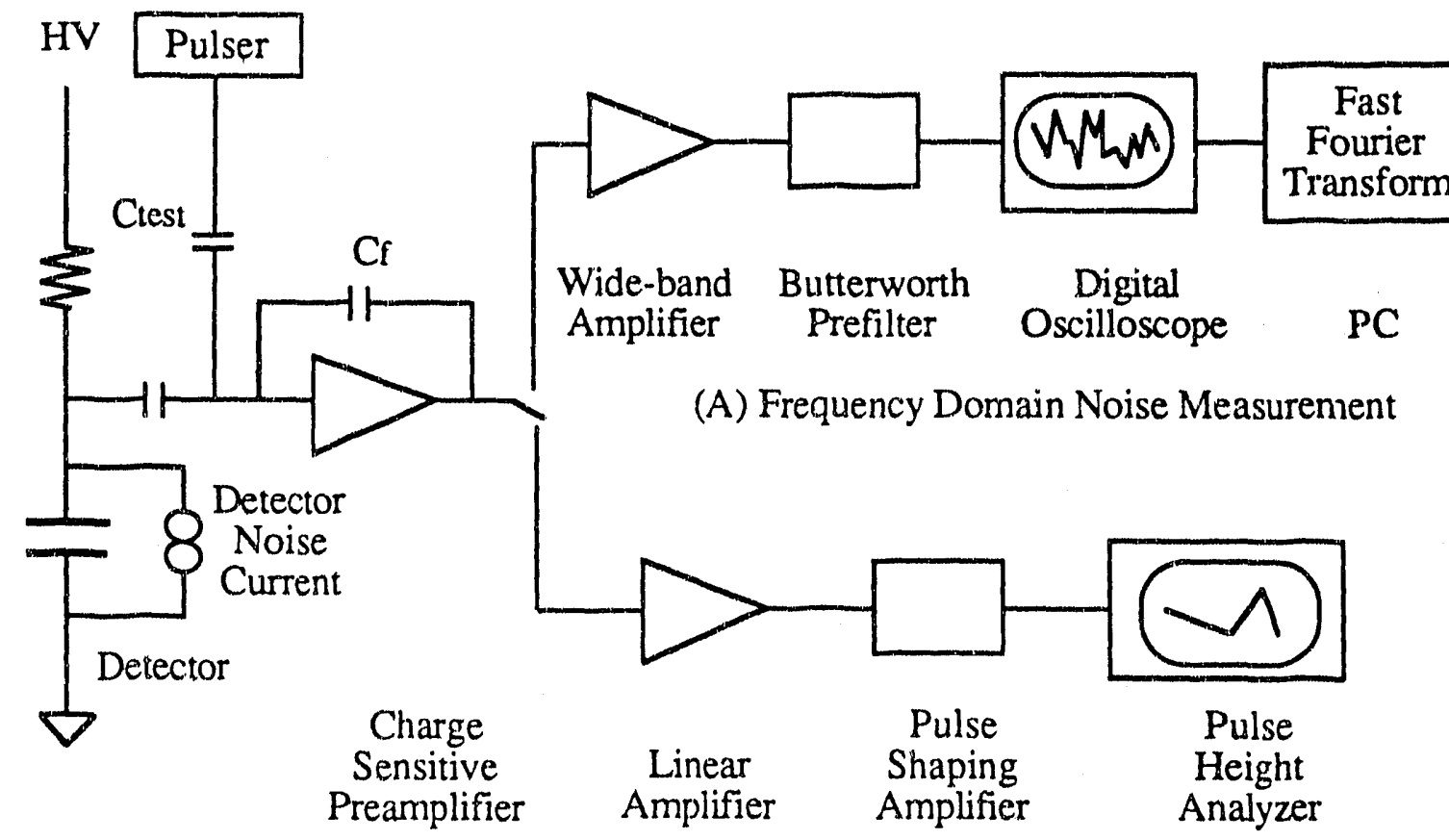

(B) Time Domain Noise Measurement

Fig. 4.10 A schematic diagram of a-Si:H detector diode noise measurement setup of (A) noise power spectrum measurement and (B) equivalent noise charge measurement. 


\section{(1) Reverse Current}

Fig. 4.11 shows a typical I-V curve of an a-Si:H pin diode ( $26 \mu \mathrm{m}$ thick) measured at room temperature and the calculation of the bulk generation current using the PooleFrenkel current model. Model calculation fits the measurements well by taking PooleFrenkel coefficient $\beta_{P F}$ as a fitting parameter. A value of 0.066 for $\beta_{P F}$ gives the best fit. We also measured the activation energy of the reverse current, $E_{a}$, which is a slope of the $\log (\mathrm{I})$ vs $1 / \mathrm{T}$ curve at each bias point. The temperature ranges between $20^{\circ} \mathrm{C}$ and $150^{\circ} \mathrm{C}$. The activation energy as a function of bias as shown in Fig. 4.12 for a typical sample diode (26 $\mu \mathrm{m}$ thick), decreases linearly with the bias rather than the square root of the bias anticipated from Poole-Frenkel barrier lowering given by.

$$
I_{P F} \propto \exp \left[\frac{-E_{a}}{k T}\right]=\exp \left[\frac{-E_{i}+\Delta E}{k T}\right]=\exp \left[\frac{-E_{i}+\beta_{P F} \sqrt{F_{0}}}{k T}\right]
$$

The larger value of $\beta_{P F}$ than the theoretical value of 0.022 suggest that the Poole-Frenkel effect may also be enhanced by a screening effect from the high density of surrounding ionized dangling bonds, i.e. clusters of defects states reduce the barrier height further.[41] In such case, the additional barrier lowering is given by $\Delta E=s F / 2$ where $s$ is the average distance between two neighboring recombination centers. 


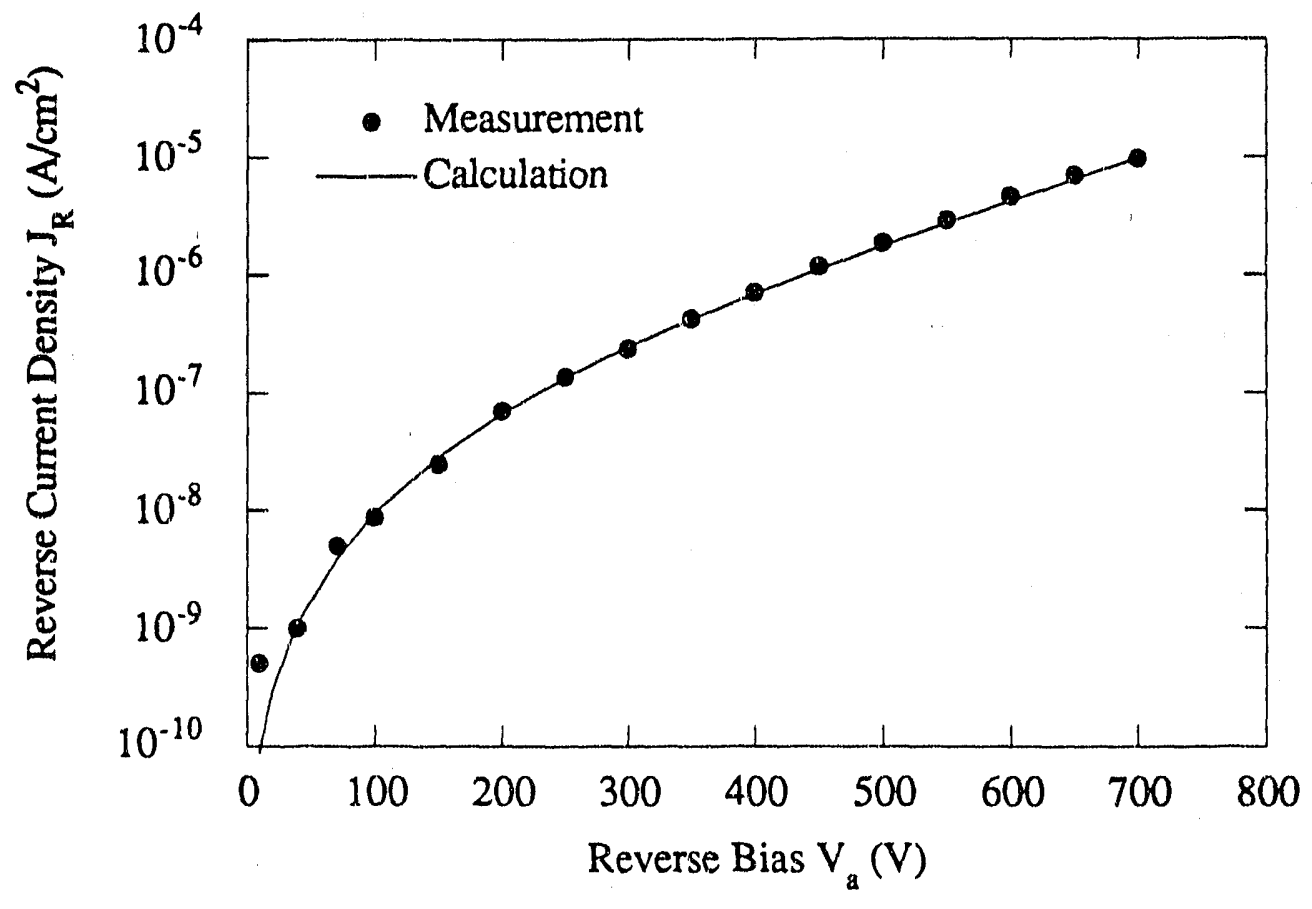

Fig. 4.11 Reverse current of a $26 \mu \mathrm{m}$ thick a-Si:H pin diode. To fit the measured data, we used $\beta_{P F}=0.066, \omega_{0}=10^{12}, E_{i}=0.9$ and $N_{t}=1.4 \times 10^{15}$.

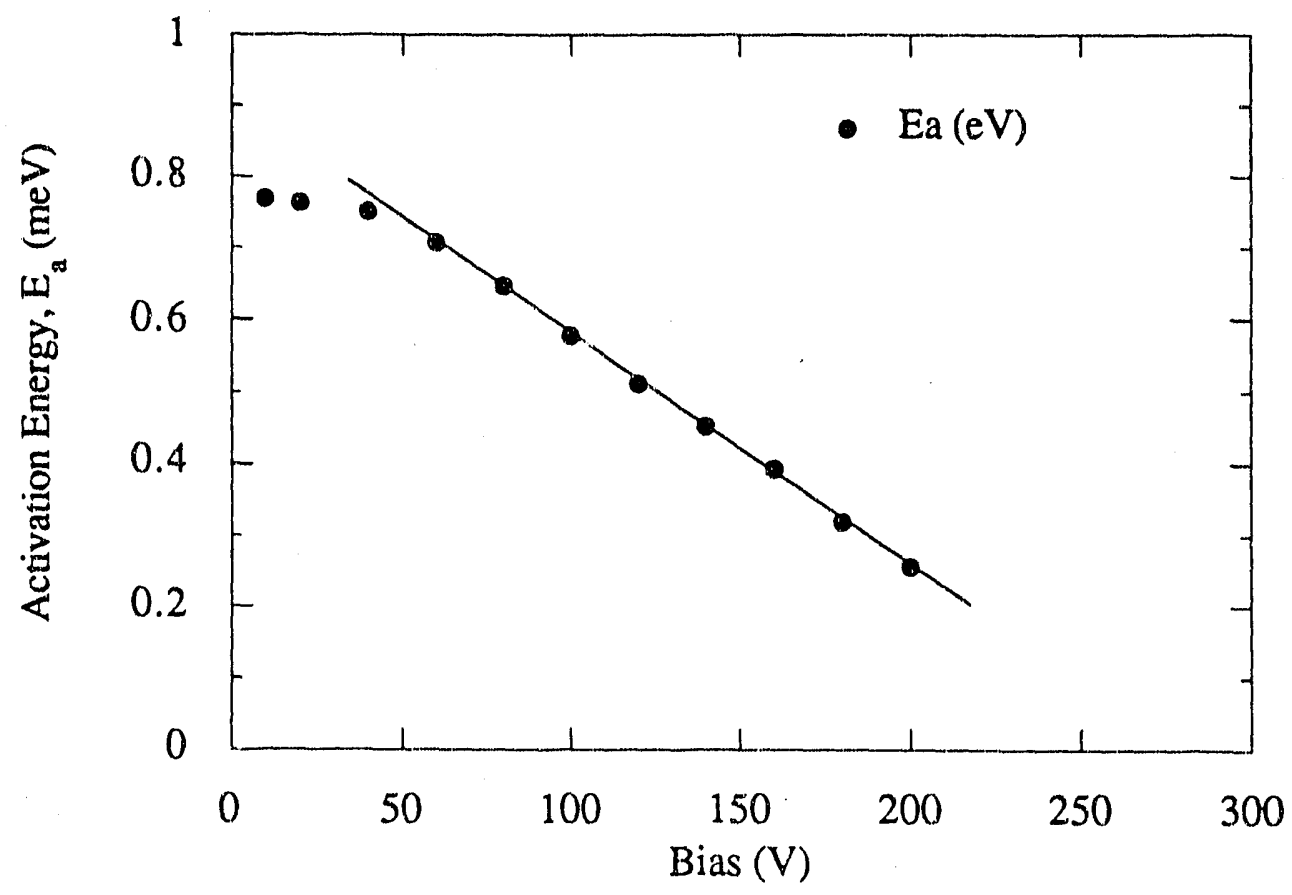

Fig. 4.12 Activation energy of reverse biased a-Si:H pin diode $26 \mu \mathrm{m}$ thick. 


\section{(2) Noise Power Spectrum}

Fig. 4.13 shows a typical output voltage noise spectra $S_{v}$ of a $26 \mu \mathrm{m}$ detector measured at various bias levels and it also shows the background noise spectrum of the amplifier itself. The spectral noise power after subtraction of the amplifier noise from the total noise is analyzed as a combination of three different noise components; $1 / \mathrm{f}$ noise and shot noise of the detector and series resistance noise based on equation (4.25). These are (a) $1 / f^{3}$ dependent component, (b) $1 / f^{2}$ dependent component and (c) frequency independent component. The magnitudes of the first and the second noise components are a function of the reverse current. The last noise component is independent of the frequency and the current.

$$
S_{v} \equiv \frac{\left\langle v_{\text {out }}^{2}\right\rangle}{\Delta f}=\frac{K_{1}\left(I_{R}\right)}{f^{3}}+\frac{K_{2}\left(I_{R}\right)}{f^{2}}+K_{3}
$$

The first two components are current dependent and therefore their physical explanations are treated as a current noise source.

The input equivalent current noise power spectra $S_{I}$ of the first two noise components are obtained by the following relation from their measured output voltage noise spectra $S_{v}$.

$$
S_{I} \equiv \frac{\left\langle i_{\text {in }}^{2}>\right.}{\Delta f}=\frac{\left(\omega C_{\text {in }}\right)^{2}}{A_{w h}^{2}} \times S_{v}=\frac{(2 \pi f)^{2}}{A_{\text {sys }}^{2}} \times S_{v}
$$

where $\omega$ is $2 \pi f, C_{i n}$ is the dynamic input capacitance of the charge-sensitive-preamplifier, $A_{w b}$ is the voltage gain of the wide-band amplifier and $A_{s y s}$ is the charge-to-voltage conversion gain of the measurement system $(A)$ which is $\sim 3 \times 10 \mathrm{~V}^{2} /$ Coul $!^{2}$.

The last component of noise spectrum is independent of the current and the frequency; therefore it can be considered as a series noise voltage source between the detector and the amplifier.

Fig. 4.14 is a schematic diagram of the detector structure and the three input equivalent noise sources represented in an equivalent circuit of the detector. 


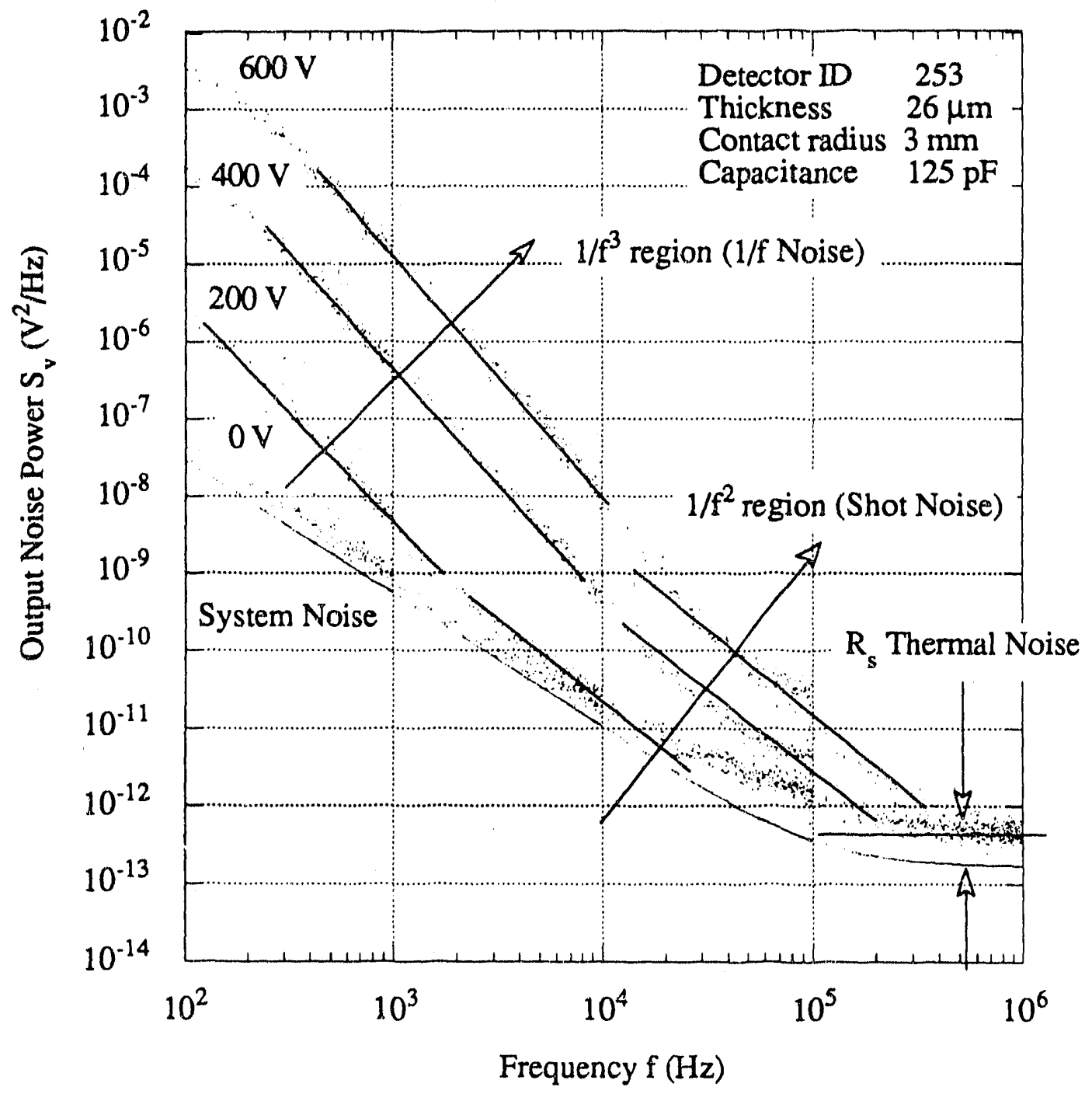

Fig. 4.13 Measured output noise power spectrum of a $26 \mu \mathrm{m}$ thick a-Si:H pin diode. $1 / \mathrm{f}^{3}$ region corresponds to $1 / \mathrm{f}$ noise of at the input stage, and $1 / \mathrm{f}^{2}$ region is shot noise and the frequency independent noise is considered as a thermal noise generated at the contacts. 


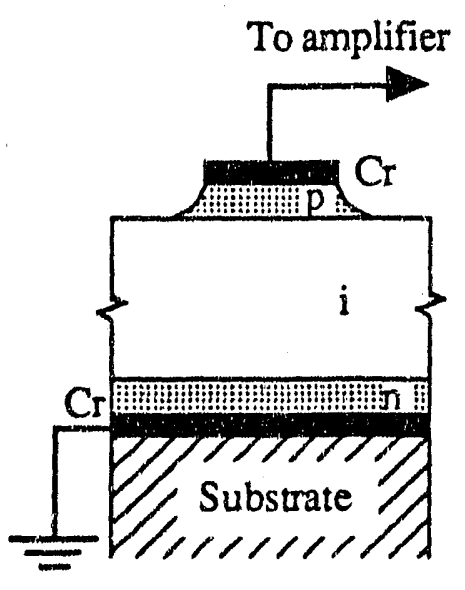

(a)

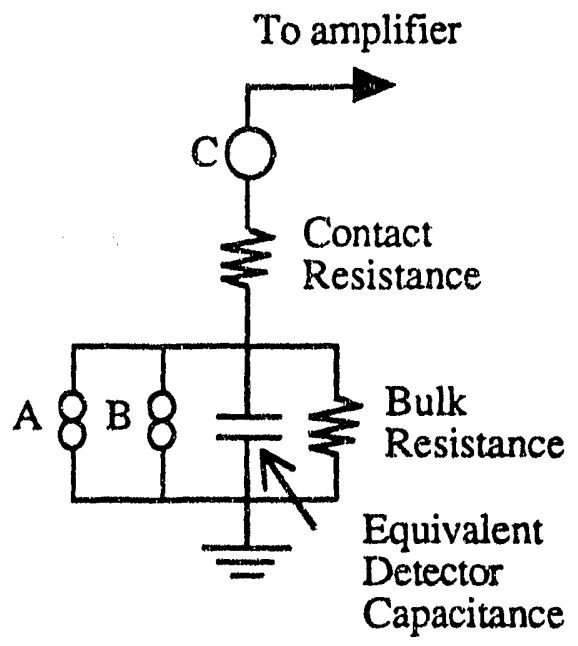

(b)

Fig. 4.14 (a) A schematic cross-sectional diagram of sample a-Si:H pin diode and (b) three noise sources in an equivalent circuit. Noise sources are A: $1 / \mathrm{f}$ noise, $B$ : shot noise and $C$ : thermal noise from contact resistance. Large value of bulk resistance doesn't contribute significant noise.

(a) $1 /$ f Noise :

The first noise component in the form of the input equivalent current noise power spectrum is inversely proportional to the frequency so it would be $1 / \mathrm{f}$ type noise. $\alpha$ was 1. It can be well expressed by the following equation,

$$
\frac{\left\langle i_{1 / f}^{2}\right\rangle}{\Delta f}=\frac{K_{0}}{f}=K_{f} \times \frac{I_{R}^{\beta}}{f}
$$

where l/f noise power coefficient, $K_{0}$, is estimated from the measured value of $K_{1}$ by equation (4.26) and is

$$
K_{0}=\left(\frac{2 \pi}{A_{\text {sys }}}\right)^{2} \times K_{1}
$$

$\beta$ is the current dependency factor and $K_{f}$ is a current independent coefficient. The measured $\beta$ has a range from $1.5 \sim 2.0$ and the $1 / f$ noise model equation derived in the previous section gives $\beta$ of -1.2 from the equation (4.19) as shown in Fig. 4.15. Fig. 4.16 shows the measured $\mathrm{K}_{\mathrm{f}}$ of various samples and the estimated values of $\mathrm{K}_{\mathrm{f}}$ calculated by the model equation. 


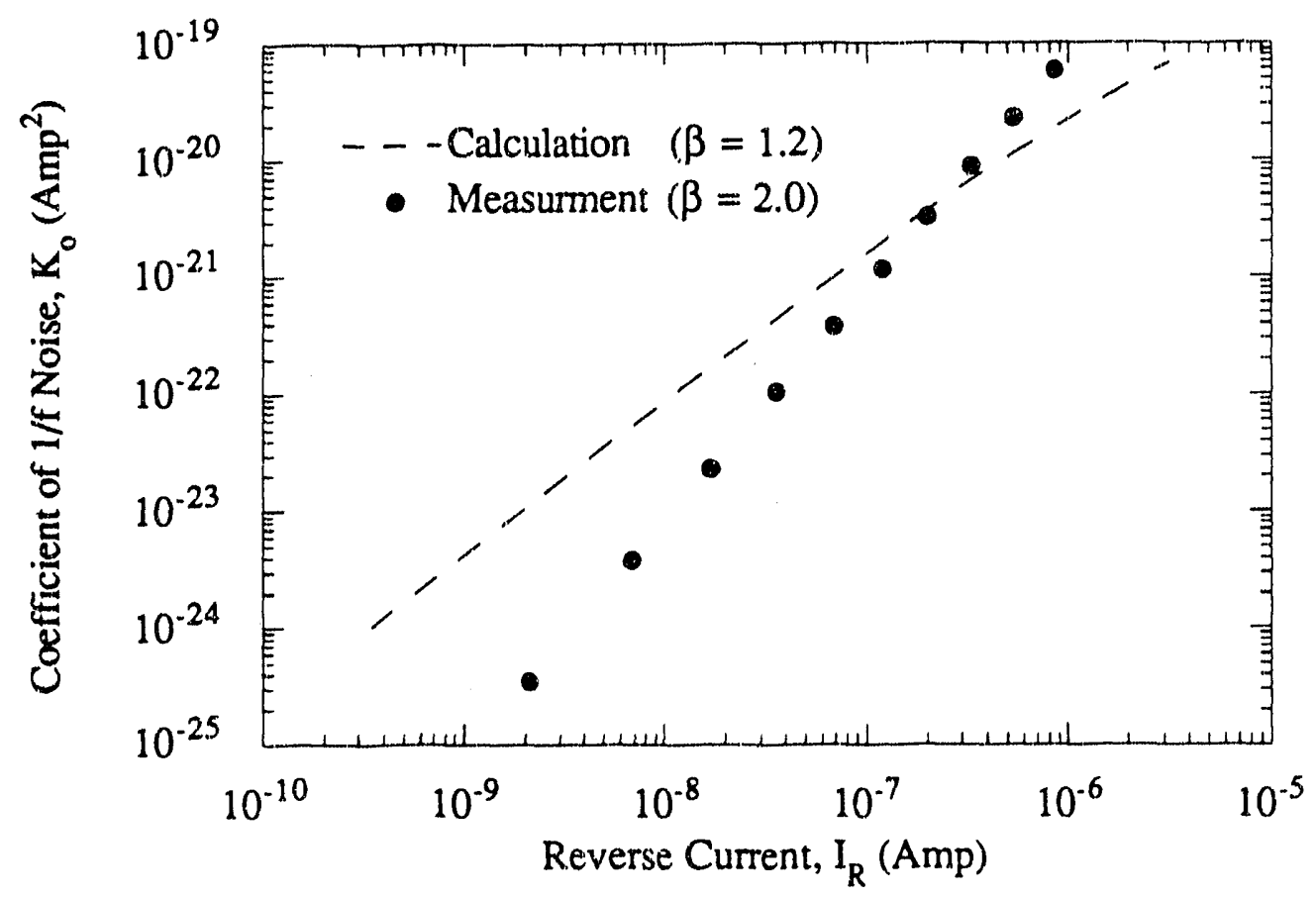

Fig. 4.15 A plot of calculated noise current power coefficient $K_{0}$ for a $26 \mu \mathrm{m}$ thick pin diode as a function of calculated Poole-Frenkel current. The calculation gives $\beta$ of 1.2 .

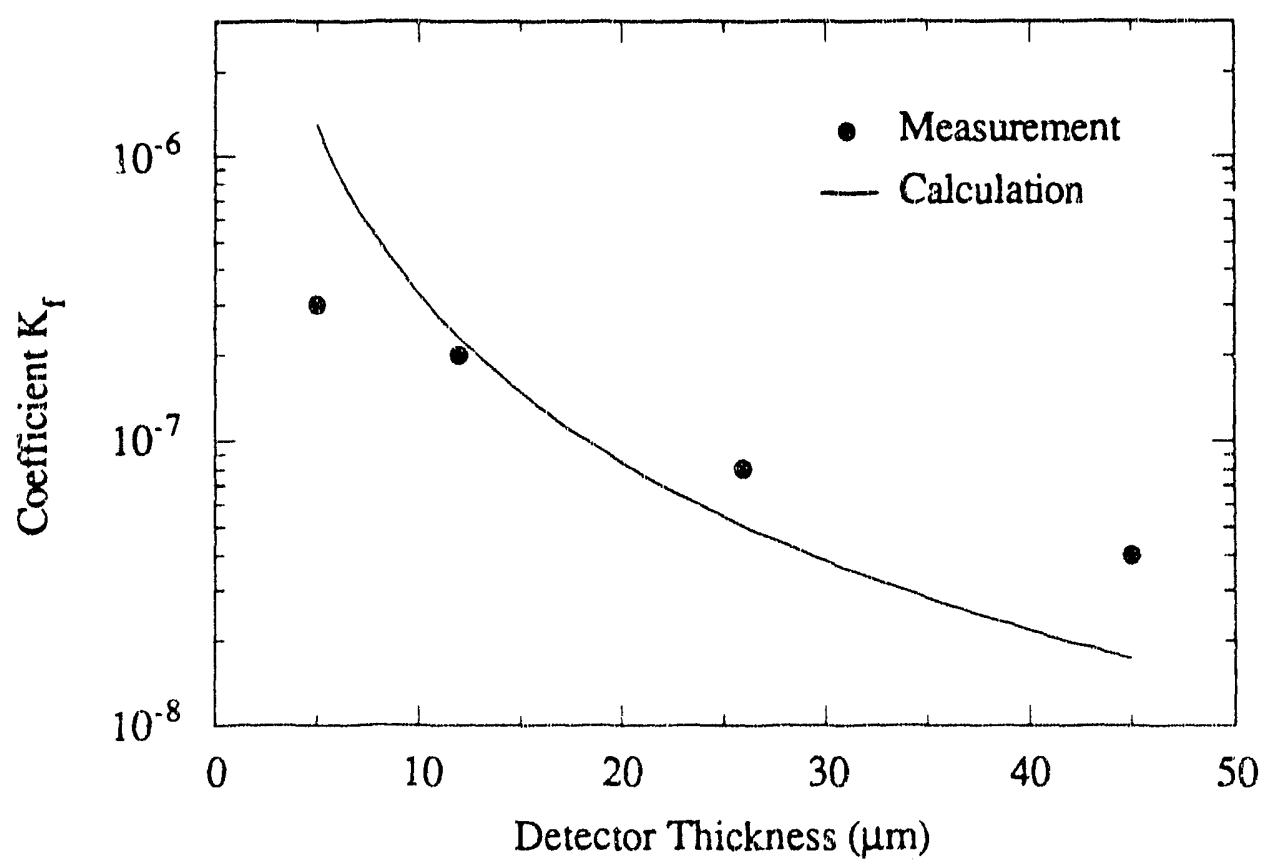

Fig. 4.16 Measured $\mathrm{K}_{\mathrm{f}}$ for a-Si:H pin diodes of various detector thickness and calculated $K_{f}$ with the assumption of $\beta=1.2$. 
(b) Shot Noise ;

The second component is shot noise and is expressed by

$$
\frac{<\mathrm{i}_{\text {shol }}^{2}>}{\Delta \mathrm{f}}=\mathrm{K}_{\mathrm{S}} \times \mathrm{I}_{\mathrm{R}}
$$

where the estimated $\mathrm{K}_{\mathrm{S}}$ from measured $\mathrm{K}_{2}$ is $\sim 2.3 \mathrm{q}$ and agrees well with the theoretical value of $2 q$ given by the equation (4.10).

\section{(c) Series Resistance Noise ;}

The last component can be represented as a series noise voltage source. From the simulation of this type of noise by a pure capacitor and a resistor, this type of noise turns out to be thermal noise from any resistive component between the detector and the amplifier. Its input equivalent noise power spectrum is given by equation (4.24)

$$
S_{\mathrm{sT}}(f)=\frac{\left\langle v_{\mathrm{SP}}^{2}\right\rangle}{\Delta \mathrm{f}}=4 \mathrm{kTR} R_{\mathrm{s}}
$$

where $4 \mathrm{kT}=1.66 \times 10^{-20}$ Volt-Coul. and $R_{S}$ is the effective series resistance between the detector and amplifier. The estimated $R_{S}$ for various samples is $100 \sim 300 \Omega$. The thermal noise comes from random thermal motion of electrons and holes in an ohmic material. The resistunce may originate from various sources, such as the contact resistance of the p- or $n$ layer to the metal electrode, the p-or n-layer itself, the sheet resistance of the metal electrode, and the resistance of the connecting wire between the detector and the amplifier, etc. One of the main contributions is the resistance of the p-layer itself, and annealing under bias can reduce this resistivity temporarily.[42] In the application of a-Si:H to solar cells, the contact resistance must be reduced to $\leq 1 \Omega$.

\section{(3) Equivalent Noise Charge}

Fig. 4. 17 and Fig. 4.18 show the measured reverse current of a typical a-Si:H pin detector diode and its equivalent noise charge measured using the setup shown in Fig. 4.10(B) as a function of bias and as a function a shaping time. Measured noise data are 
fitted by the sum of four noise components estimated using equations $(n=1)$ in Table 4.3 and the parameters evaluated from the noise power spectra. As we see in these measurernents, the derived equations for equivalent noise data for a-Si:H detectors fit them very well so they can be used for the design of large-area devices using appropriate scaling.

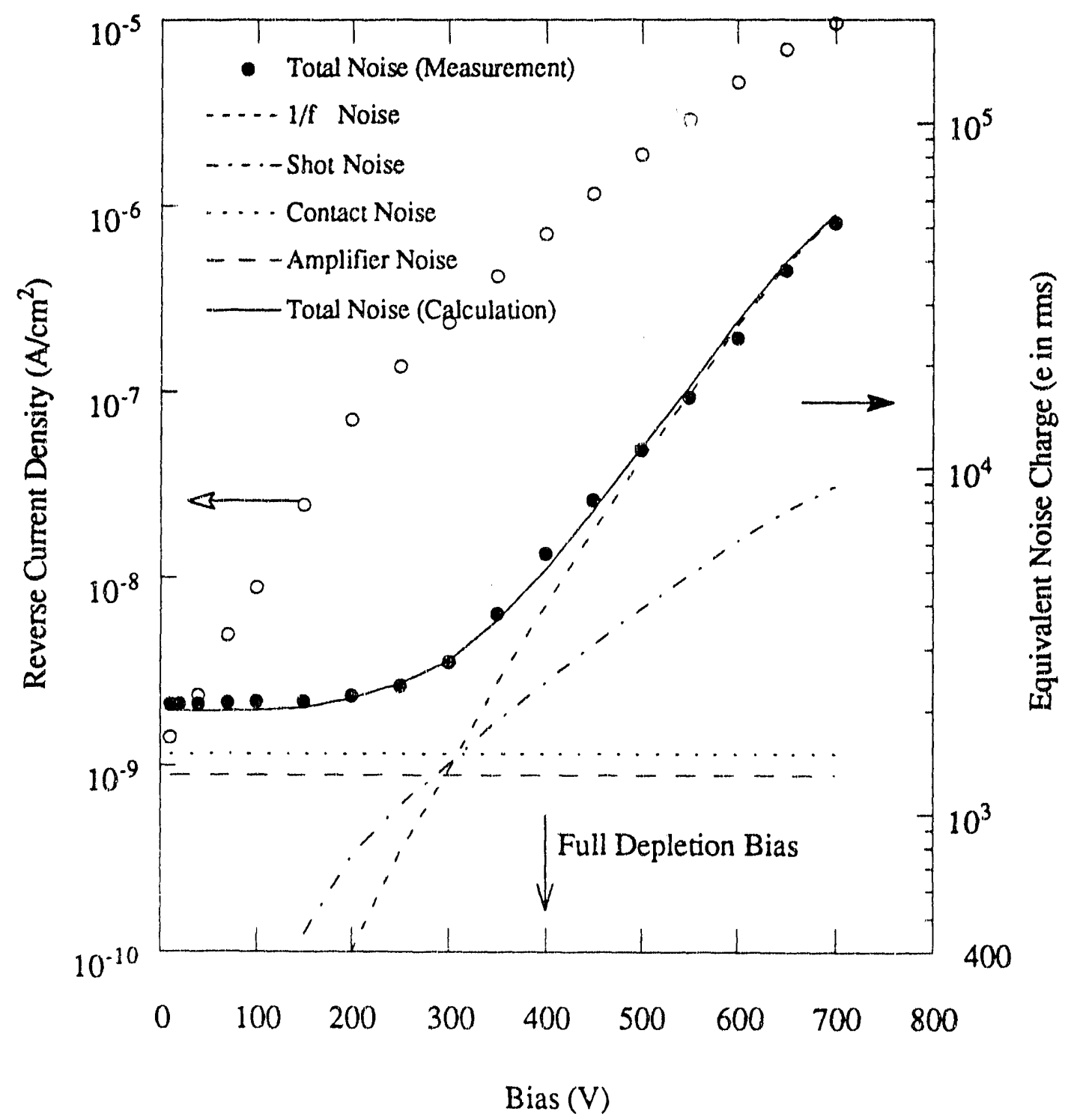

Fig. 4.17 Reverse current and equivalent noise charge measurement of a reverse biased 26 $\mu \mathrm{m}$ thick a-Si:H pin diode and calculated ENC of (a) $1 / \mathrm{f}$ noise, (b) shot noise, (c) contact noise, (d) amplifier noise and the total noise(solid line). Shaping time of $2.5 \mu \mathrm{sec}$ is used. 


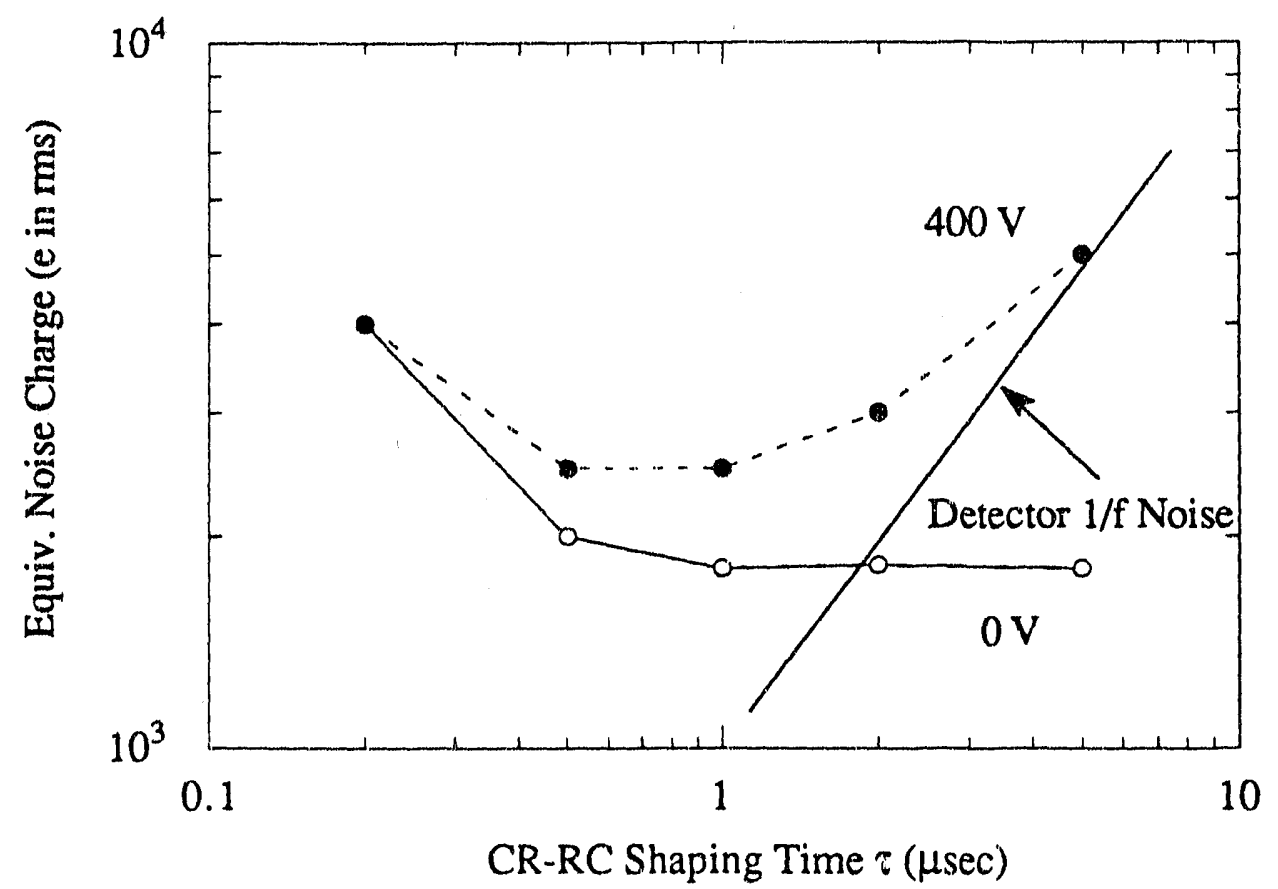

Fig. 4.18 Measure noise of a $26 \mu \mathrm{m}$ a-Si:H pin diode as a function of CR-RC shaping time. Detector $1 / \mathrm{f}$ noise is shown as a linearly increasing part of the total noise at high bias.

\subsubsection{I-V and Noise Power Spectrum of TFTs}

Noise of a-Si:H and poly-Si TFTs were measured at a shielded probe station using the setup drawn in Fig. 4.19. Details of the measurement set-up are given in reference [43].

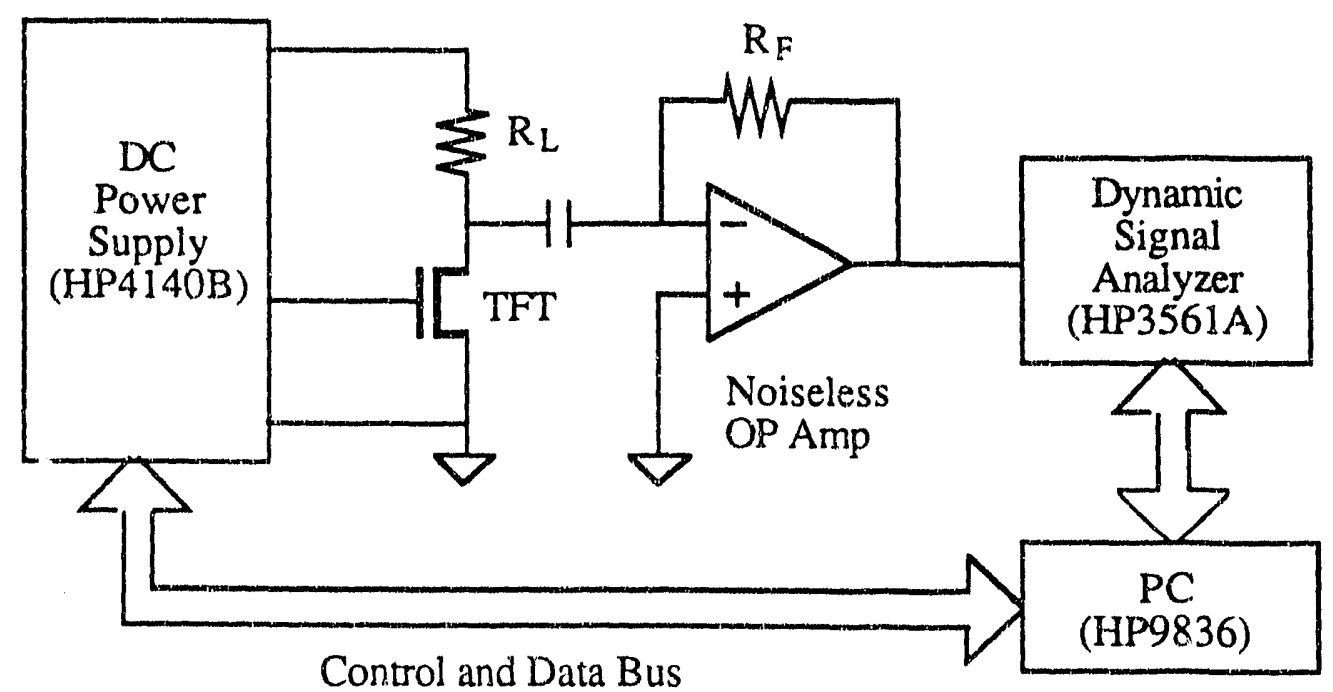

Fig. 4.19 A schematic diagram of TFT Noise measurement system. (Courtesy of Hung) 
Fig. $4.20 \sim$ Fig. 4.22 show typical I-V and noise spectra of a-Si:H TFTs and Fig. 4.23 and Fig. 4.24 show I-V curve and measured $1 /$ f noise spectrum poly-Si TFTs. The aSi:H and poly-Si TFTs tested have very large $1 / f$ type noise which dominates over a large frequency range up to $\sim \mathrm{MHz}$. The thermal noise was not observed. The $1 / \mathrm{f}$ noise coefficient $\mathrm{K}_{\mathrm{t}}$ of poly-Si TFT is $\sim 100$ times smaller than that of a-Si:H TFT shown by fitting the data of noise power spectrum into the equation (4.22). Also the field effect mobility of poly-Si TFT is $~ 200$ times higher than that of a-Si:H TFT as shown from the I$\mathrm{V}$ measurements and the analysis of equation (4.20). Therefore poly-Si TFTs are more attractive candidates for the front-end amplifier which should be fabricated on the same substrate together with the a-Si:H pin detector layer. In terms of $1 / \mathrm{f}$ noise, $\mathrm{p}$-channel polySi TFT seems a better choice than n-channel poly-Si TFT.

Table 4.3 summarizes some basic parameters of a-Si:H and poly-Si TFTs made at two different annealing temperature.

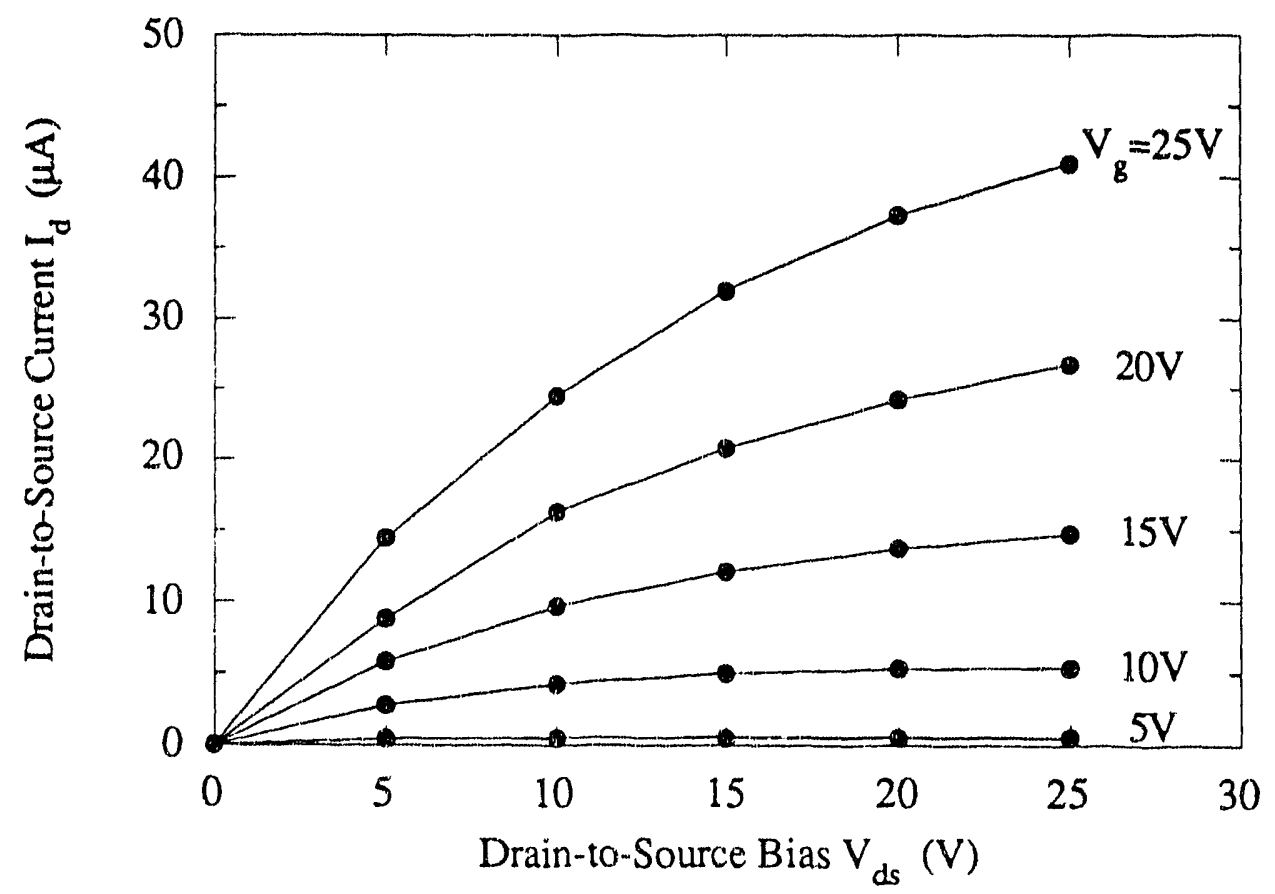

Fig. 4.20 Typical I-V curves of a-Si:H TFT made at Xerox. W/L $=128 / 16 \mu \mathrm{m}$. 


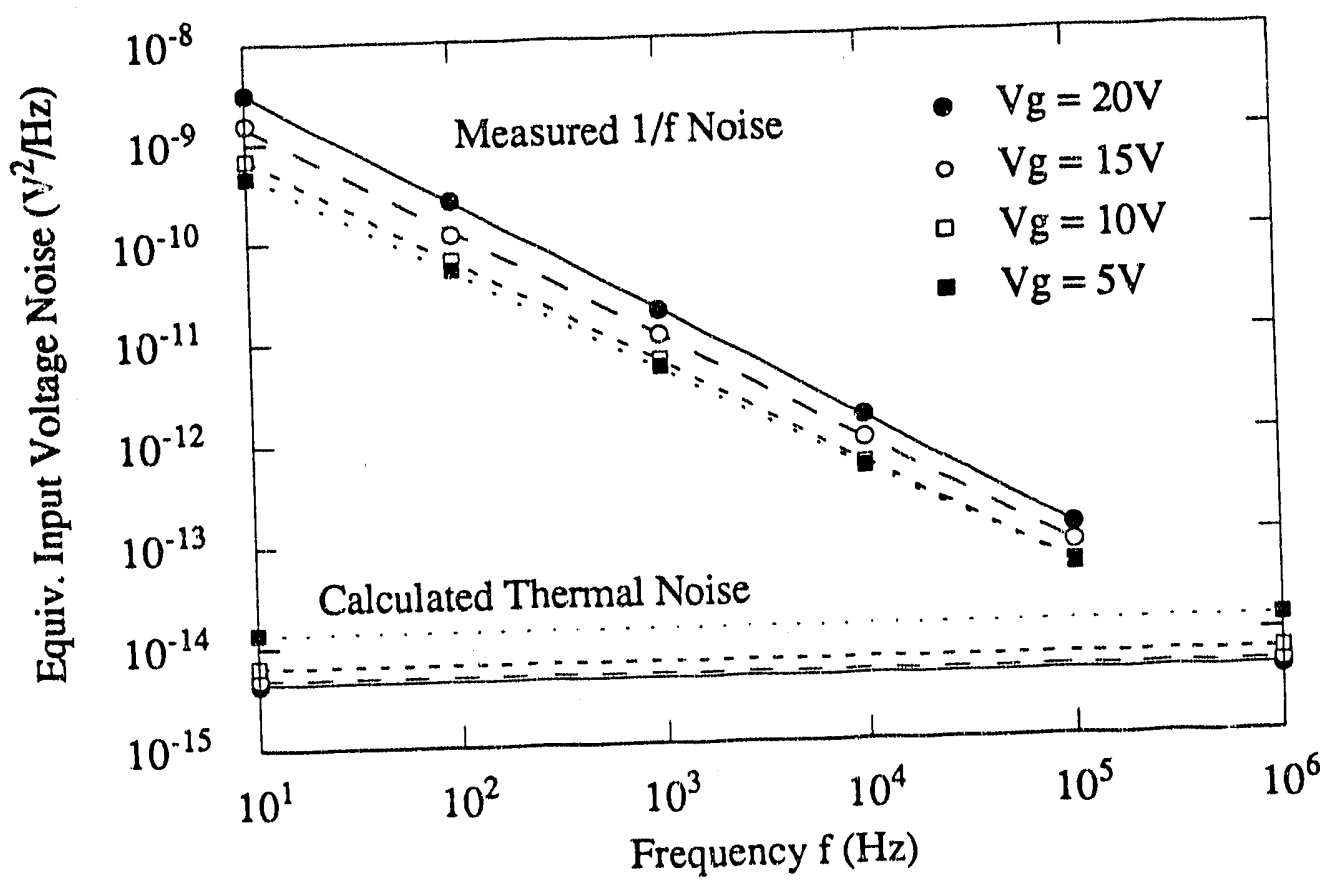

Fig. 4.21 Measured 1/f noise spectrum of a-Si:H TFT made at Xerox. W/L $=128 / 16 \mu \mathrm{m}$ and the applied drain-to-source bias is $20 \mathrm{~V}$. Thermal noise is estimated from the measured values of transconductance $\mathrm{g}_{\mathrm{m}}$.

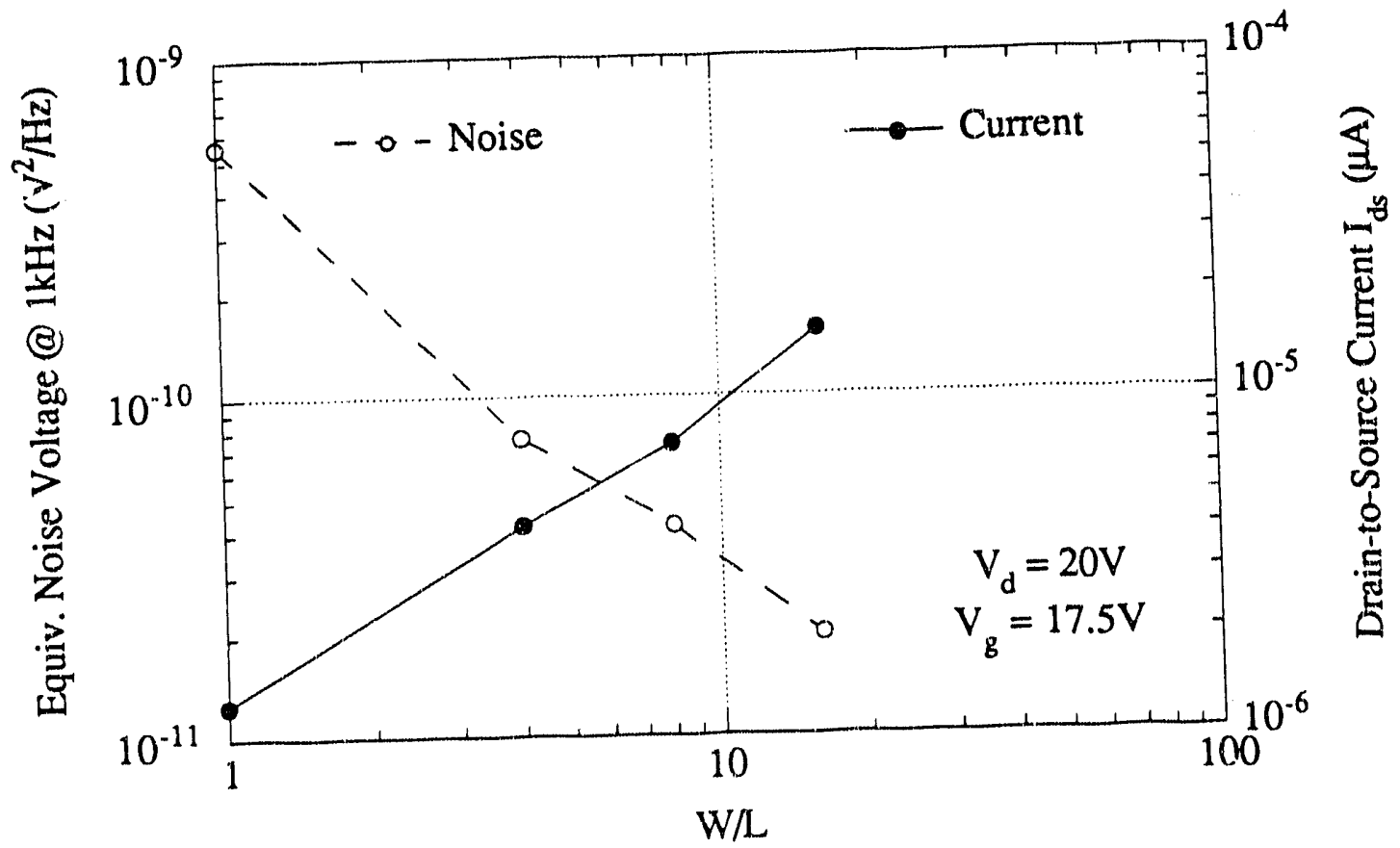

Fig. 4.22 Magnitude of $1 / \mathrm{f}$ noise power at $1 \mathrm{kHz}$ and drain-to-source current of a-Si:H TFTs as a function of channel dimension $W / L$. 


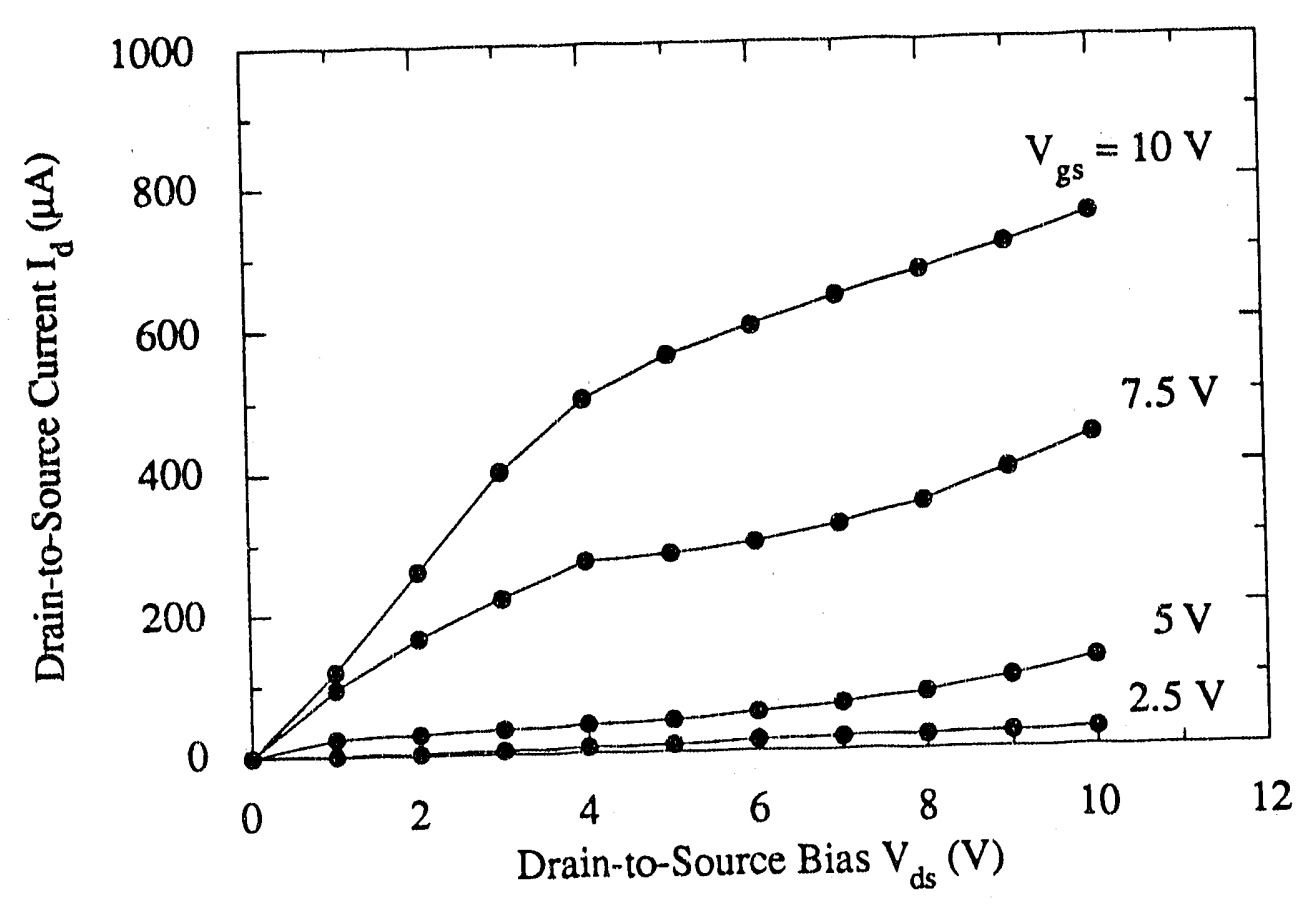

Fig. 4.23 I-V curves of $900^{\circ} \mathrm{C}$ annealed n-channel poly-Si TFT made at Xerox. W/L = $50 / 5 \mu \mathrm{m}$.

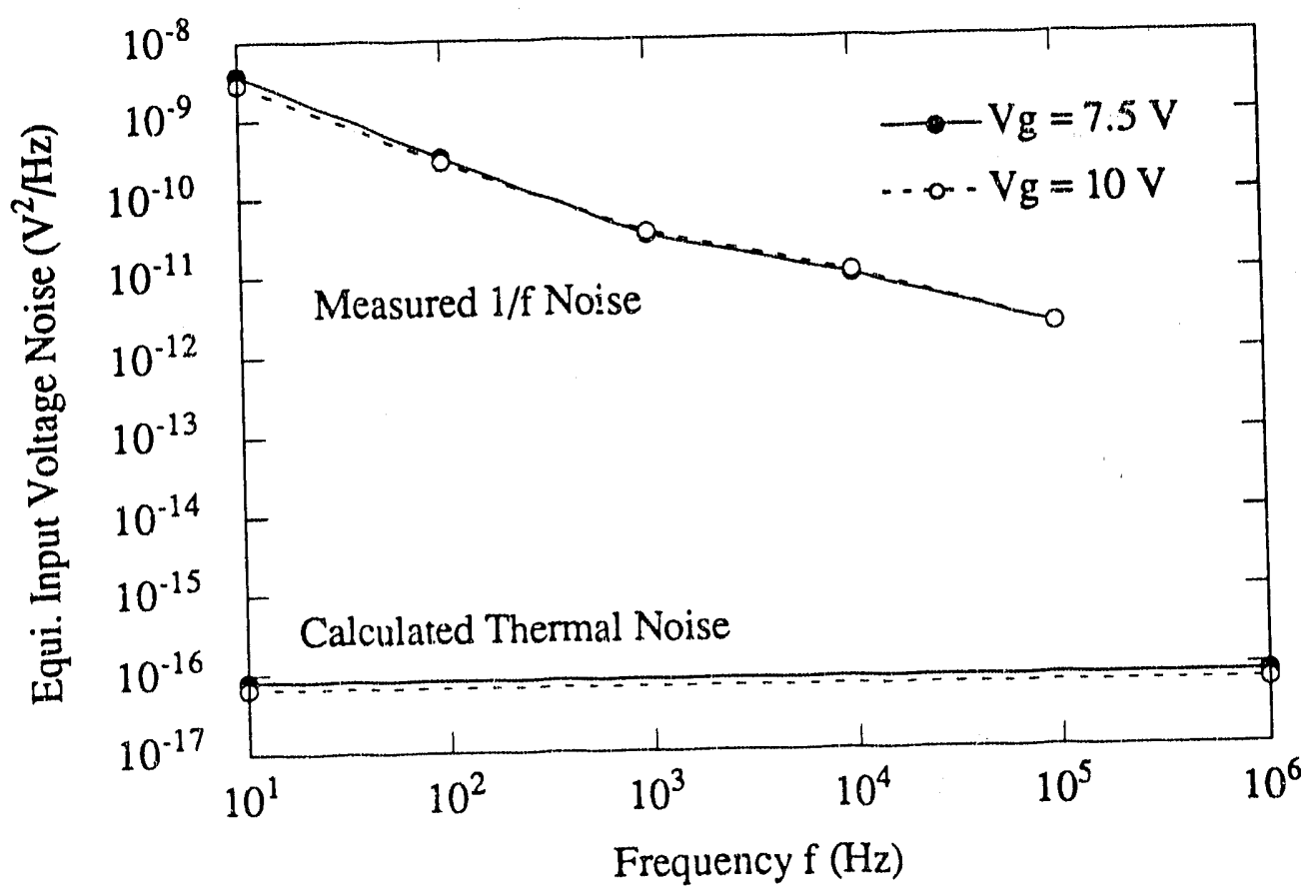

Fig. 4.24 Measured $1 /$ f noise spectrum of $900^{\circ} \mathrm{C}$ annealed $n$-channel poly-Si TFT. W/L = $50 / 5 \mu \mathrm{m}$ and the applied drain-to-source bias is $10 \mathrm{~V}$. Thermal noise is estimated by the measured transconductance $\mathrm{g}_{\mathrm{m}}$. 
Table 4.3 A summary of basic features of a-Si:H and poly-Si TFTs

\begin{tabular}{|c|c|c|c|c|c|c|}
\hline Type & $\begin{array}{c}\mu_{\mathrm{fe}} \\
\left(\mathrm{cm}^{2} / \mathrm{Vsec}\right)\end{array}$ & $\begin{array}{c}\mathrm{V}_{\mathrm{T}} \\
(\mathrm{Volt})\end{array}$ & $\begin{array}{c}\mathrm{L} \\
(\mu \mathrm{m})\end{array}$ & $\begin{array}{c}\text { Insulator } \\
(\mu \mathrm{m})\end{array}$ & $\alpha$ & $\begin{array}{c}\mathrm{K}_{\mathrm{t}} \\
\left(10^{-20} \mathrm{VC}\right)\end{array}$ \\
\hline a-Si:H TFT & $\begin{array}{c}0.25 \sim \\
0.35\end{array}$ & $2.5 \sim 3$ & $>5$ & $\begin{array}{c}\mathrm{Si}_{3} \mathrm{~N}_{4} \\
0.2 \sim 0.3\end{array}$ & $\sim 1.0$ & 1.2. \\
\hline $\begin{array}{c}\text { a-Si:H } \\
\mathrm{VTFT}^{*}\end{array}$ & $\begin{array}{c}0.25 \sim \\
0.35\end{array}$ & - & $\sim 0.5$ & $\begin{array}{c}\mathrm{Si}_{3} \mathrm{~N}_{4} \\
(\mathrm{NA})\end{array}$ & - & - \\
\hline $\begin{array}{c}600^{\circ} \text { poly-Si } \\
\text { n \& p-ch }\end{array}$ & $40 / 15$ & $1 \sim 3$ & $>3$ & $\begin{array}{c}\mathrm{SiO}_{2} \\
0.1 \sim 0.2\end{array}$ & - & - \\
\hline $\begin{array}{c}900^{\circ} \text { poly-Si } \\
\text { n-ch TFT }\end{array}$ & $\sim 95$ & $1 \sim 2.5$ & $>3$ & $\begin{array}{c}\mathrm{SiO}_{2} \\
0.1 \sim 0.2\end{array}$ & $\sim .78$ & $.01 \sim .02$ \\
\hline $\begin{array}{c}900^{\circ} \text { poly-Si } \\
\text { p-ch TFT }\end{array}$ & $\sim 50$ & $2 \sim 3$ & $>3$ & $\begin{array}{c}\mathrm{SiO}_{2} \\
0.1 \sim 0.2\end{array}$ & $\sim .8$ & $.007 \sim .01$ \\
\hline
\end{tabular}

* See reference for vertical TFT.[44]

\subsection{Discussion}

In the a- $\mathrm{Si}: \mathrm{H}$ radiation detectors which are reverse biased pin diodes, dark reverse current is modelled as a field enhanced thermal generation current due to the Poole-Frenkel effect. Using the number fluctuation model we derived the $1 / \mathrm{f}$ noise of a reverse-biased aSi:H pin diode. Measured reverse current curves for $5 \sim 50 \mu \mathrm{m}$ thick diodes are fitted well by the model equation. However the $1 / \mathrm{f}$ noise model has a discrepancy with the measured data in terms of the current dependency factor $\beta$ showing 1.2 instead of the measured value of $\sim 2$.

Measured noise power spectra of diodes are analyzed as a sum of three components; $1 / \mathrm{f}$ noise, shot noise and contact noise. The first two noise components are due to the fluctuations of dc reverse current, and the contact noise can be from any series resistive components between the detector capacitance and the preamplifier. Resistivity of 
the p-layer itself and the electrode metal are probably the origin of an effective contact resistance of $\sim 200 \Omega$.

Measured I-V curves for sample TFTs show that the noise in both TFTs is dominated by a $1 / \mathrm{f}$ noise component up to $\sim \mathrm{MHz}$ range, but poly-Si TFT has the merit of achieving higher gain and lower $1 / f$ noise amplifier than a-Si:H TFT.

Using the noise power spectra of detector diodes measured in the frequency domain, the equivalent noise charge in the time domain after a $C R-(R C)^{n}$ shaping filter are derived and compared to the measurements done by conventional distributed nuclear electronics. At low bias, series contact resistance noise is important but as the bias increase shot noise and $1 / \mathrm{f}$ noise become dominant. The $1 / \mathrm{f}$ noise in the detector can be reduced by a factor of 2 using a CR-(RC) ${ }^{4}$ filter compared to a CR-RC filter and it can also be reduced further by decreasing the shaping time. Time variant active filters such as a gated-integrator with double correlation sampling can avoid the switching transients and reduce $1 / \mathrm{f}$ noise from TFT amplifiers. However since the thermal noise contribution may increase, the timing design must be done optimally in the direction to reduce the total noise of the system.

The derived expressions of equivalent noise charges from various noise sources of a detector and TFT combination as given in the Table 4.3, will be useful when we calculate the total noise of the detector-amplifier system. Optimization of signal-to-noise ratio can be done by choosing a proper combination of operation parameters such as the size of the detector and the front-end TFTs and the operation bias levels, the shaping time and shaping filter circuit, etc. 


\section{References}

[1] U. Fano, "Ionization Yield of Radiation. II. The Fluctuations of the Number of Ions," Phys. Rev. 72, 26 (1947)

[2] H. R. Zulliger and D. W. Aitken, "Fano Factors Fact and Fallacy," IEEE Trans. Nuc. Sci., NS-17, 187 (1970)

[3] W. Shockley, "The Theory of p-n Junctions in Semiconductors and p-n Junction Transistors," Bell Syst. Tech. J., 28, 435 (1949)

[4] C. R. Crowell and S. M. Sze, "Current Transport in Metal-Semiconductor Barriers," Solid State Electron., 9, 1035 (1966)

[5] C. T. Sah, R. N. Noyce and W. Shockley, "Carrier Generation and Recombination in p-n Junction and p-n Junction Characteristics," Proc. IRE, 45, 1228 (1957)

[6] W. Shockley and W. T. Read, "Statistics of the Recombination of Holes and Electrons," Phys. Rev., 87, 835 (1952)

[7] R. A. Street, "Thermal Generation Current in Hydrogenated Amorphous Silicon pi-n Structures," Appl. Phys. Lett., 57, 1334 (1990)

[8] J. Frenkel, "On Pre-Breakdown Phenomena in Insulators and Electronic Semiconductors," Phys. Rev., 54, 647 (1938)

[9] E. Rosencher, V. Mosser and G. Vincent, "Transient-Current Study of FieldAssited Emission from Shallow Levels in Silicon," Phys. Rev. B, Vol. 59, 1135 (1984)

[10] G. Vincent, A. Chantre and D. Bois, "Electric Field Effect on Thermal Emission of Traps in Semiconductor Junctions," J. Appl. Phys., 50, 5484 (1979)

[11] E. N. Koral, "Ionization of Impurity States in Semiconductors by an Electric Field," Sov. Phys. Solid State, 19, 1327 (1977)

[12] R. A. Street, "Dopant and Defect States in a-Si:H," Phil. Mag. B, Vol. 52, 235 (1985)

[13] A. van der Ziel, Noise in Solid State Devices and Circuits, John Wiley \& Sons, p. $19(1986)$

[14] A. van der Ziel, Noise in Solid State Devices and Circuits, John Wiley \& Sons, p. 145 (1986)

[15] F. N. Hooge and L. K. J. Vandamme, "Lattice Scattering Causes 1/f Noise," Phys. Lett., Vol. 66A, 315 (1978)

[16] A. L. McWhorter, Semiconductor Surface Physics, (Ed. R. H. Kingston) University of Pennsylvania Press, p. 207 (1956) 
[17] F. N. Hooge, "1/f Noise," Physica, 83B, 14 (1976)

[18] T. G. M. Kleinpenning, "1/f Noise in p-n Junction Diodes," J. Vac. Soc. Tech. A, Vol. 3, $176(1985)$

[19] X. C. Zhu and A. van der Ziel, "The Hooge Parameters of $n^{+}-p-n$ and $p^{+}-n-p$ Silicon Bipolar Transistors," IEEE. Trans. Electron. Dev., ED-32, 658 (1985)

[20] K. H. Duh and A. van der Ziel, "Hooge Parameters for Various FET Structures," IEEE. Trans. Electron. Dev., ED-32, 662 (1985)

[21] F. M. Klaassen, "Characterization of Low 1/f Noise MOS Transistors," IEEE Trans. Electron. Dev., ED-18, 887 (1971)

[22] A. D'Amico, G. Fortunato and C. M. van Vliet, "Noise Measurements in Thin Films of Amorphous Silicon," J. Non-Cryst. Sol., 77 \& 78, 499 (1985)

[23] F. Z. Bathaei and J. C. Anderson, "Electrical Noise Measurements in Intrinsic Amorphous Silicon,". Phil. Mag. B, Vol. 55, 87 (1987)

[24] C. Parman and J. Kakalios, "Non-Linear 1/f Noise in Amorphous Silicon," Phy. Rev. Lett., Vol. 67, 2529 (1991)

[25] W. K. Choi, A. E. Owen, P. G. LeComber and M. J. Rose, "Exploratory Observation of Random Telegraphic Signals and Noise in Homogeneous Hydrogenated Amorphous Silicon," J. Appl. Phys., 68, 120 (1990)

[26] F. Z. Bathaei and J. C. Anderson, "Flicker Noise in Hydrogenated AmorphousSilicon Schottky Diodes," Phil. Mag. B, Vol. 57, 259 (1988)

[27] G. Cho, J. Drewery, I. Fujieda, S. N. Kaplan, V. Perez-Mendez, S. Qureshi and R. A. Street, "Measurements of $1 / \mathrm{f}$ Noise in a-Si:H pin Diodes and Thin Film Transistors," Mat. Res. Soc. Symp. Proc., Vol. 192, 393 (1990)

[28] A. C. Hourd and W. E. Spear, "Determination of the Extended-state Electron Mobility in a-Si," Phil. Mag. B, Vol. 51, L13 (1985)

[29] S. Moruzumi, H. Kurihara, T. Takeshita, H. Oka and K. Hasegawa, "Completely Integrated Contact-Type Linear Image Sensor," IEEE Trans. Electron. Dev., ED$32,1546(1985)$

[30] A. Lewis, T. Y. Huang, R. H. Bruce, M. Koyanagi, A. Chiang and I-Wei Wu, "Polysilicon Thin Film Transistors for Analogue Circuit Applications," IEDM-88, p. 264 (1988)

[31] S. Seki, T. Unagami and B. Tsujiyama,"High Transconductance Si-TFTs Using $\mathrm{Ta}_{2} \mathrm{O}_{5}$ Film as Gate Insulator," 16th Conf. Solid State Devices and Materials., p. $563(1984)$

[32] Y. Shiraki and E. Maruyama "Poly-Si TFTs and Their Applications to Liquid Crystal Display," Amorphous Semiconductor Technologies and Devices, Vol. 6, p. 266 (1983) 
[33] A. Lewis, I-Wei Wu, T. Y. Huang, A. Chiang and R. H. Bruce, "Active Matrix Liquid Crystal Display Design Using Low and High Temperature Processed Polysilicon TFTs," IEDM-90, p. 843, (1990)

[34] T. I. Cox, V. G. I. Deshmukh, J. R. Hill, H. C. Webber, N. G. Chew and A. G. Cullis, "Electrical and Structural Properties of Pulse Laser-Annealed Polycrystalline Silicon Films," IEEE Trans. Electron. Dev., ED-30, 737 (1983)

[35] A. Van der Ziel, "Thermal Noise in Field-Effect-Transistors," Proc. IRE, Vol. 50, $1808(1962)$

[36] P. R. Gray and R. G. Meyer, Analysis and Design of Analog Integrated Circuit, 2nd Ed., John Wiley \& Sons, p. 666 (1977)

[37] F. S. Goulding and D. A. Landis, "Signal Processing for Semiconductor Detectors," IEEE Trans. Nuc. Sci., NS-29, 1125 (1982)

[38] G. R. Hopkinson and D. H. Lumb, "Noise Reduction Techniques for CCD Image Sensors," J. Phys., E 15, 1214 (1982)

[39] R. W. Brodersen and S. P. Emmons, "Noise in Buried Channel Charge-Coupled Devices," IEEE J. Solid-State Circuits, SC-11, 147 (1976)

[40] R. J. Kansy, "Response of a Correlated Double Sampling circuit to $1 / \mathrm{f}$ Noise." IEEE J. Solid-State Circuits, SC-15, 373 (1980)

[41] R. M. Hill, "Poole-Frenkel Conduction in Amorphous Solids," Phil. Mag., Vol, 23, $59(1971)$

[42] G. Cho, S. Qureshi, J. S. Drewery, T. Jing, S. N. Kaplan, H. Lee, A. Mireshghi, V. Perez-Mendez and D. Wildermuth, "Noise in a-Si:H p-i-n Detector Diodes," Presented at IEEE Nuc. Sci. Symp., Santa Fe, NM., Nov. 4, 1991.

[43] K. K. Hung, P. K. Ko, C. Hu and Y. C. Cheng, "Flicker Noise Characterisics of Advanced MOS Technologies," IEDM-88, p. 34 (1988)

[44] M. Hack, J. G. Shaw and M. Shur, "Novel a-Si:H TFTs for Use in Large Area Microelectronics," Mat. Res. Soc. Symp. Proc., Vol. 118, p. 207 (1988) 


\section{Chapter 5 Detector Readout Electronics}

\subsection{Introduction}

Any of the readout schemes mentioned in Chapter 2 can be applied to positionsensitive detector made of a-Si:H, however we shall limit our discussion to the linescanning method for 2-d pixel detectors only because it can be applied in the widest range of applications. Since a-Si:H detectors can be made together with a-Si:H or poly-Si TFTs on the same substrate, the readout electronics for 2-d a-Si:H pixel detectors is preferably made from these materials. A-Si:H TFTs and a-Si:H pin diodes have been successfully used as a readout switch for the line-scanning method for light imaging.[1,2] This scheme can also be applied to a-Si:H radiation detectors. For single-particle or low-intensity $\mathrm{x}$-ray 2-d pixel detectors, it is also necessary to amplify the signal at the pixel level before readout by the external processing electronics.

In this chapter, after a brief description of transistor- and diode-switch readout of aSi:H 2-d pixel detectors, we will discuss two aspects of pixel readout electronics in some detail; an a-Si:H diode-readout and a poly-Si pixel amplifier.

\section{2 a-Si:H Pixel Detector Electronics}

\subsubsection{Transistor-Switch Readout}

A transistor readout scheme of stored charges in a solid photodiode was originally suggested by Weckler in 1967.[3] Recently Street et al.[1] have made a-Si:H pin photodiode arrays with a single switching-transistor/pixel readout scheme. Its schematic is shown in Fig. 5.1. Each element consists of a photodiode and a switching a-Si:H TFT for the readout. The diode is reverse biased through a common bias line. The drain of the 
readout transistor is connected to a pixel diode and the source is connected to a common data line. Finally the gate electrode is connected to a common gate lines.

Signal charge is accumulated and stored in each pixel diode during a period of the scanning cycle. When the clock pulse generator sends a gate pulse to each row $(x)$ of pixels in sequence, signals from the pixels in the row are readout directly through each data line (y) by an external parallel processor. The readout speed is determined by the conductance of the TFT. The processor accepts a set of analog data in sequence and produces digital pulses having informations about the position and signal size of pixels. This data can then Lis stored into a memory or displayed on a screen. The scanning cycle for a pixel in a scanning readout method is divided into an integration time, $T_{i}$, and a readout time, $T_{r}$.

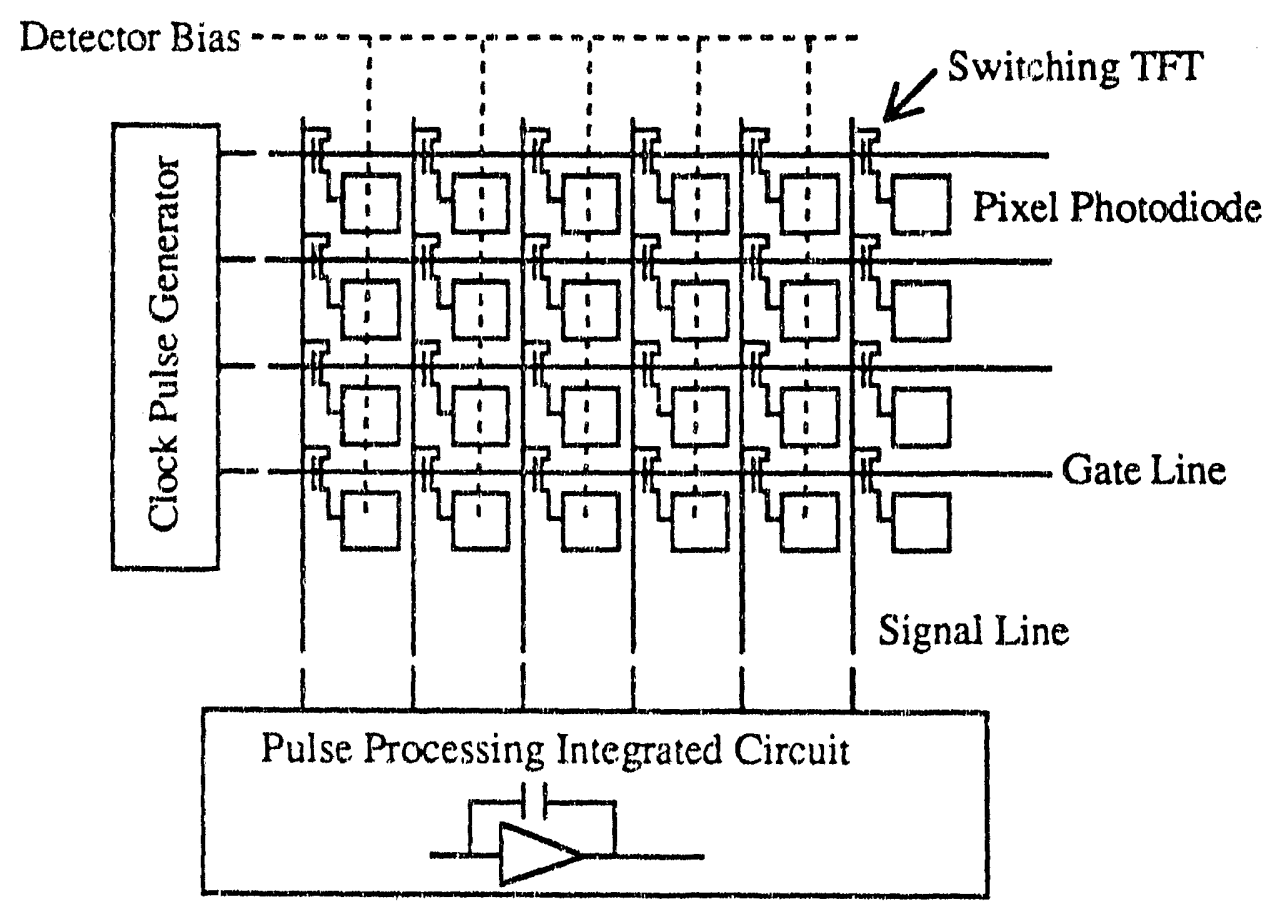

Fig. 5.1 A-Si:H pin photodiode pixel array with a-Si:H TFT switching TFTs for readout data line by line. (Courtesy of Xerox PARC) 
For the optimum design of this system several considerations are required; (a) The reverse leakage current of the photodiode must be small to avoid signal loss during the integration time. (b) For a fast readout, the on-time resistance of a-Si:H TFT should be small enough to transfer the signal charge at sufficiently high rate. For example, a drainsource resistance of $1 \mathrm{M} \Omega$ (on state) with a pixel detector of $1 \mathrm{pF}$ will produce $1 \mu \mathrm{sec}$ readout speed. (c) For high efficiency of the signal transfer, the ratio of the dynamic input capacitance of the preamplifier to the capacitance of a pixel diode should be large. (d) To get a higher sensitivity and wide range of dynamic response, the system noise should be minimized. Noise from the readout TFT can be treated as a series resistance noise and $1 / \mathrm{f}$ noise.

\subsubsection{Diode-Switch Readout}

A diode can act as an electronic switch because it conducts under forward bias and does not under reverse bias condition. Therefore a pixel readout switch can be made by a diode connected back-to-back with a pixel diode. Yamamoto et al.[2] have made an a-Si:H 2-D image sensor for document page reading using a single readout switching diode and a pixel diode. Its schematic is shown in Fig. 5.2.

The switching diode is normally off and the pixel diode is reverse biased during an integration time, $T_{i}$. Signal charges produced by the incident radiation or flux during this time are accumulated on the capacitance of the pixel diode. During the readout time, $T_{r}$, the switching diode is forward biased by applying an appropriate sign of pulsed bias (called a gate pulse) and the charge stored on the pixel diode is discharged. Integration of the discharging current at a charge integrator (charge-sensitive preamplifier) produces an output pulse. The discharging rate is determined by the product of the capacitance of the pixel diode and the forward resistance of the switching diode. The minimum readout time is determined by the discharging rate and the process time of the output pulse at the external 
circuit. The optimum integration time should be decided by the number of pixel columns and the signal loss rate due to the leakage current of the pixel diode.

The diode switching readout, compared to the transistor switching readout, has disadvantages, for example, highly nonlinear characteristics in the forward bias region may produce severe variations in forward conductance from pixel-to-pixel. However, it also has some advantages. Its construction is much simpler than that of a TFT. Also its readout speed can be faster than TFTs. And since it does not use any insulating layer (c.f. gate insulator in TFTs) it may have superior radiation resistance than a TFT readout scheme. Because of these benefits diode switching has also been studied extensively for application to flat-panel liquid crystal display devices. Initially metal-insulator-metal (MIM) diodes such as tantalum oxide and silicon nitride were used. [4] However, they have a rather poor on/off current ratio $\left(10^{3} \sim 10^{4}\right)$ compared to a-Si:H TFTs. Recently a-Si:H pin diodes have been used as switches for liquid crystal displays and showed a very high on/off ratio $10^{11} \cdot[2]$

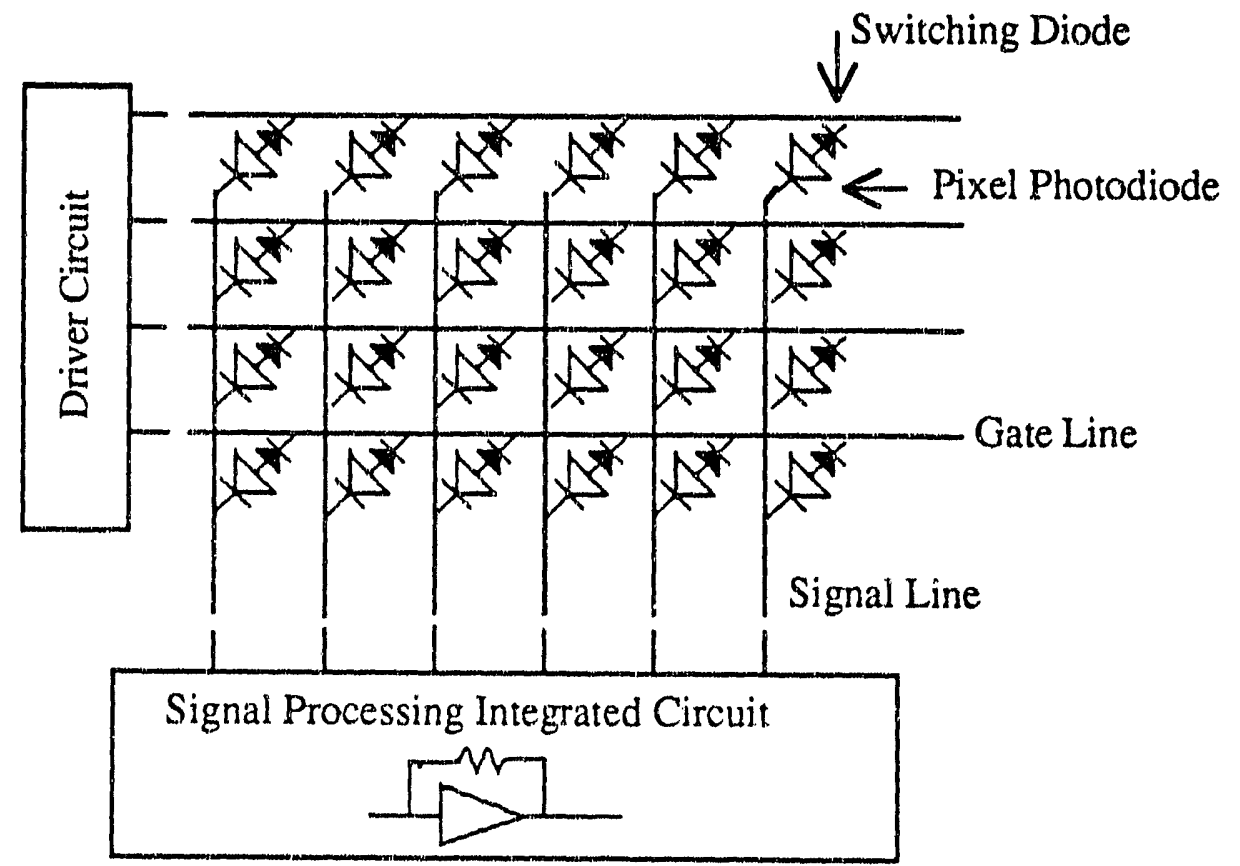

Fig. 5.2 A schematic diagram of 2-d pixel signal readout using a single diode. 
In the case of radiation detectors, due to the relatively small signal compared to the large light signal in document reading, the single diode scheme has several disadvantages. For example, (a) a large feed-through transient at the time of the on-off transition of the switching diode can interfere with, and screen the signal current and (b) the operating bias point of the pixel detector diode is not controllable but is determined solely by the reverse characteristics of the pixel and switching diodes.

linstead of a single diode, two diodes can be used to perform a similar switching function (double diode switch). [4] Fig. 5.3 shows a schematic of a unit cell composed of two switching diodes and a pixel diode. The switching diodes are connected back-to-back and are normally off during the charge integration time, $T_{i}$. During the readout time, $T_{r}$, both diodes are forward biased at the same time by the application of appropriate gate voltages (positive and negative respectively), to discharge the stored signal charges in the pixel diode.

The advantage of this scheme is (a) cancellation of the feed-through transient charges from gate pulses and (b) control of the reverse operating bias level of the pixel diode by changing the ground level of both gate pulses. The main drawback in the two diode readout is that a larger number of interconnections are needed because each pixel row requires two gate lines.

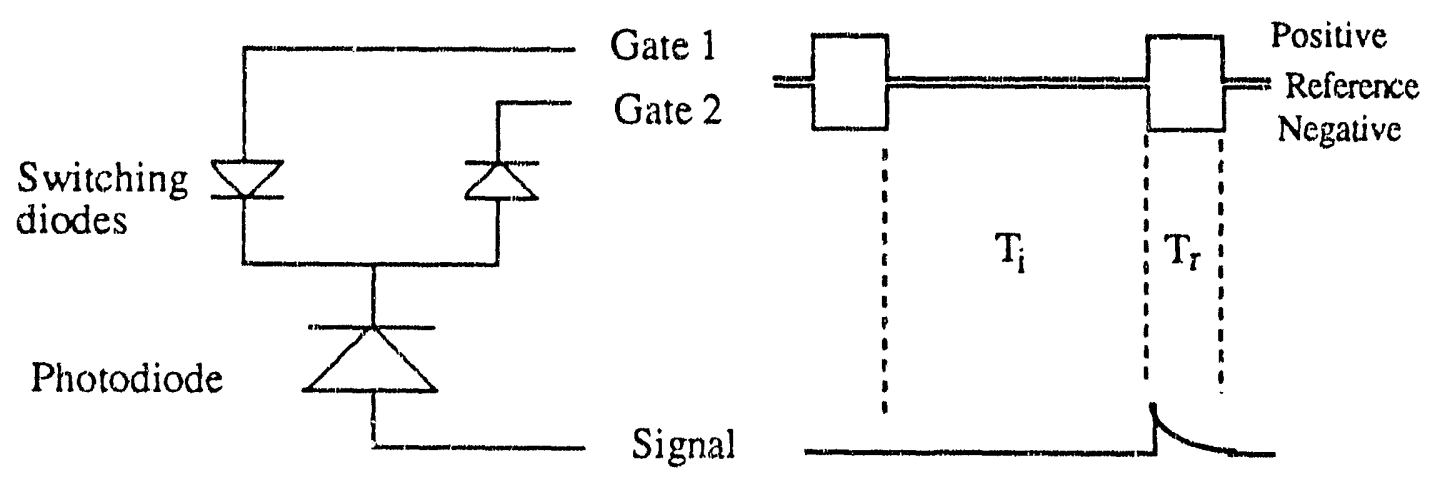

Fig. 5.3 A Schematic diagram of an unit pixel detector with back-to-back readout diodes and a timing diagram showing the integration time $T_{i}$ and the readour time $T_{r}$. 


\subsubsection{Pixel-Level Signal Amplification}

Since signals produced in amorphous silicon radiation detectors are generally small, especially in the case of single particle detection, pixel-level amplification is needed before the signal from 1-d strip or 2-d pixel detector array is read-out by the external circuitry in order to reduce stray effects, such as transfer loss of signal, and noise pick-up at data lines crossing large-area detector arrays. The pixel electronics must perform signal amplification, holding signal data, and sending the signal to a common data line as shown in Fig. 5.4.

To implement these electronic functions for a large-area 2-d pixel array of a-Si:H detectors, poly-Si TFT technology seems to be the most appropriate and natural choice because it has compatibility together with amorphous silicon in making large-area devices on the same substrate. Also it has better electronic characteristics than a-Si:H TFTs, as described in Chapter 4. This technology is still under intensive development in the linear image sensor or active matrix LCD industry. However in those applications, the TFTs are used principally as switching elements rather than as analog amplifiers, so it is important to investigate the analog characteristics of the TFTs and their limitations as well as their applicability for our purpose.

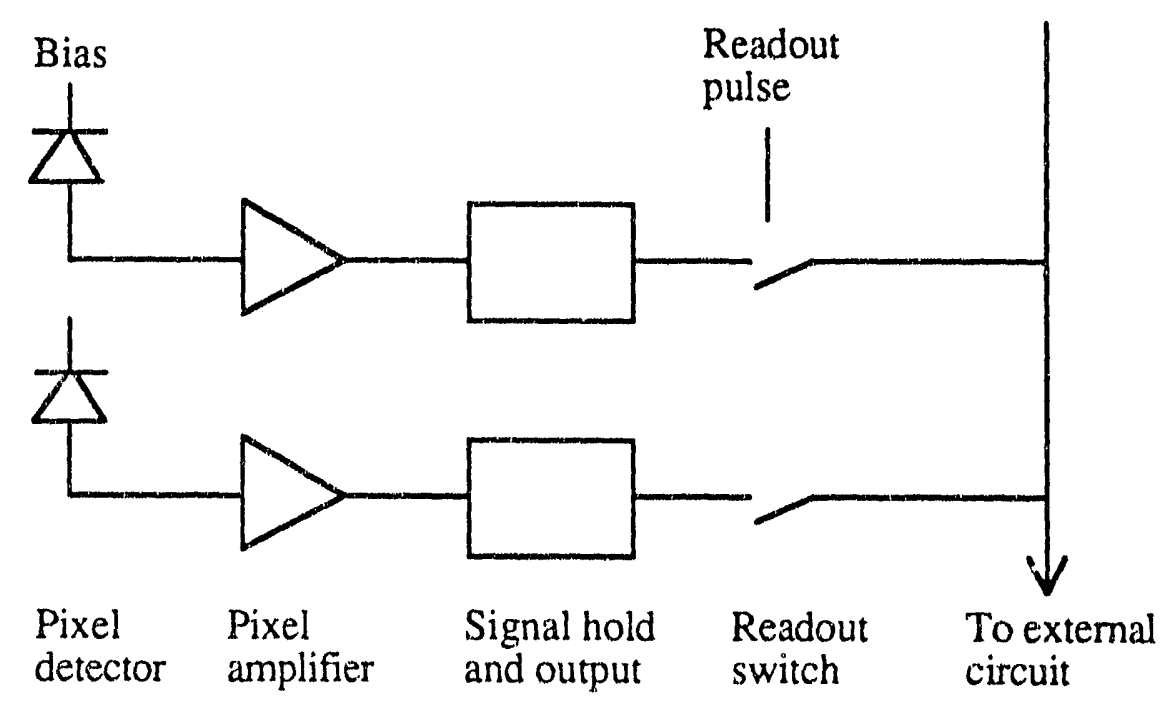

Fig. 5.4 A schematic diagram of pixel detector and pixel level electronics consisting of an amplifier, signal hold and readout switch. 


\subsection{An a-Si:H Diode-Switch Readout}

\subsubsection{Design}

An array of photodiodes ( $3 \times 3$ array of $1 \times 1 \mathrm{~mm}$ pin diode) with two back-toback coupled switching diodes was made in order to test the double diode readout scheme. The main objectives were (a) to measure the loss rate of the signal between the incidence time of the radiation and the readout time, (b) to measure the readout speed. and (c) to study the cancellation effect of the positive and negative feed-through transients. The size of the switching diodes was decided arbitrarily to have an area of $100 \mu \mathrm{m} \times 100 \mu \mathrm{m}$ which is $1 \%$ of the area of the pixel detector diode.

\subsubsection{Fabrication}

Photodiodes and switching diodes were fabricated in the same deposition process on the same substrate as shown in the Fig. 5.5.

Initially a $\mathrm{Cr}$ layer $30 \mathrm{~nm}$ thick was evaporated on a glass substrate. The chrome was then etched using the first mask to produce the boltom contact layers. Then a-Si:H pin diodes were made by PECVD. The i-layer is $2 \mu \mathrm{m}$ thick and the heavily doped $\mathrm{p}$ - and $\mathrm{n}$ layers are each $50 \mathrm{~nm}$ thick. Top metal contacts of the diodes were made by evaporating a $\mathrm{Cr}$ layer $30 \mathrm{~nm}$ thick. Then the second mask was used to pattern the top $\mathrm{Cr}$ layer and to remove the a-Si:H layers back to the substrate or the bottom $\mathrm{Cr}$ layer. An insulating, or passivation, layer of polyimide was spun-on and baked. Using the third mask, contact holes were made through the polyimide onto the bottom $\mathrm{Cr}$ layer. Finally, an Al layer was deposited and patterned using the fourth mask to draw interconnection lines and test pads. The Al layer also covers the whole area of the switching diodes in order to block the incident test light. One $\mu \mathrm{m}$ thick polyimide layer is transparent to visible light. The fabrication process is summarized in Table 5.1. Except for the deposition of a-Si:H layers 
(done at LBL), the other processes were all done at the Micro Electronics Lab in UC Berkeley.

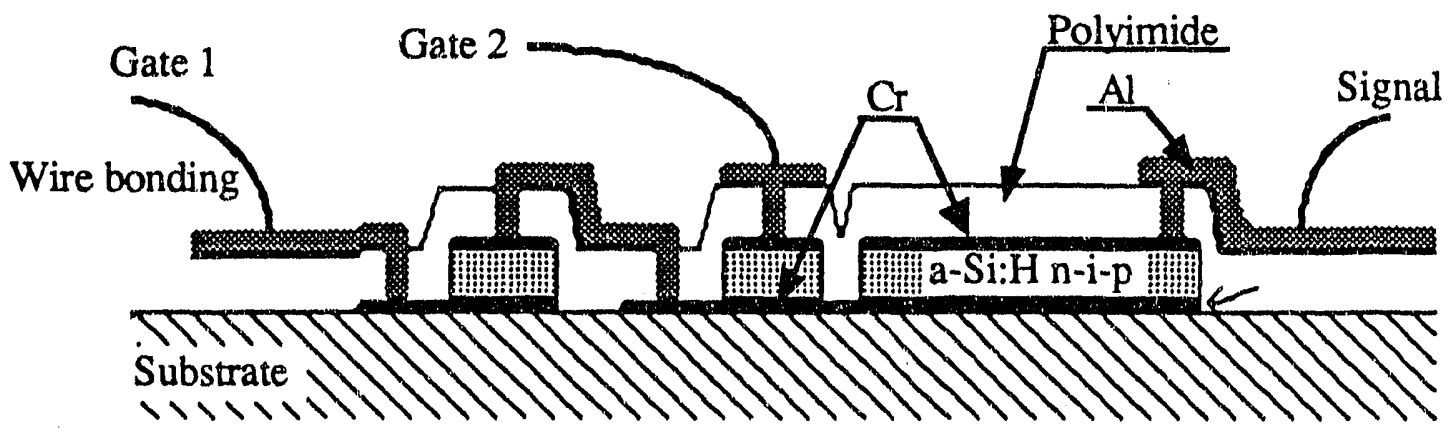

Switching diodes Photodiode

Fig. 5.5 A cross-sectional view of a two-diodes switching readout made at LBL.

Table 5.1 Integration process for a two-diodes switch coupled to pixel diodes

\begin{tabular}{|c|c|c|}
\hline Step No & Step & Target thickness \\
\hline 1 & Sputtering of bottom Cr layer & $50 \mathrm{~nm}$ \\
\hline 2 & Wet etching of bottom Cr layer with MASK-1 & \\
\hline 3 & PECVD of a-Si:H n-i-p layers & $50 \mathrm{~nm} / 2 \mu \mathrm{m} / 50 \mathrm{~nm}$ \\
\hline 4 & Sputtering of top Cr layer & $30 \mathrm{~nm}$ \\
\hline 5 & Wet etching of top Cr layer with MASK-2 & \\
\hline 6 & Dry etching of a-Si:H layers with MASK-2 & \\
\hline 7 & Spin-coating of polyimide & \\
\hline 8 & Wet etching of holes in polyimide with MASK-3 & \\
\hline 9 & Sputtering of Al layer & \\
\hline 10 & Wet etching of Al layer with MASK-4 & \\
\hline 11 & Cutting and packaging & \\
\hline 12 & Wire bonding & \\
\hline
\end{tabular}




\subsubsection{Test and Results}

First we measured the forward and reverse current characteristics of the switching diodes and photodiodes. Both diodes showed the same characteristics and a typical curve of current normalized to the area is shown in Fig. 5.6. The current on-off ratio at biases of $3 \mathrm{~V}$ and $0 \mathrm{~V}$ was typically $10^{5}$. The dynamic resistance of the forward biased switching diodes at $3 \mathrm{~V}$ was found to be $\sim 7 \mathrm{M} \Omega$ from Fig. 5.6. Under a dc reverse bias of $\sim 5 \mathrm{~V}$, some diodes showed the surface breakdown.

Then we simulated radiation detection using an LED of $880 \mathrm{~nm}$ wave-length as the radiation source in the measurement set-up shown in Fig. 5.7. A reverse operation bias of the pixel photodiode was set by changing the bias $V_{\mathrm{r}}$ at a large resistor $R_{L}(>\sim 10 \mathrm{M} \Omega$ ) rather than changing the effective ground potential of the two gate pulses. Thin pixel photodiodes $(<2 \mu \mathrm{m})$ can work at $\mathrm{V}_{\mathrm{r}}=0 \mathrm{~V}$ because of their built-in potential. A short pulse of light was shone on the pixel diode. After a time delay, $T_{d}$, positive and negative gate pulses were applied to the two switching diodes respectively. The discharging current of the accumulated charge in the photodiode was integrated by a conventional chargesensitive amplifier and the output pulse of a CR-RC shaping amplifier was measured by an oscilloscope.

From measurements of feedthrough transients directly at the summing node of two switching diodes when two gate pulses (positive and negative step pulse) were applien to the other nodes of the diodes, we found out that the transient charges could be cancelled up to $\sim 99 \%$ of the feedthrough from a single-diode readout. The cancellation was limited by mismatch in shapes of the two pulses and mismatch in impedances of the two diodes and associated connections. With the present devices, the feedthrough from the diode gate pulses produced a pedestal that was $\sim 90 \%$ of the full peak height. However it was reproducible from pulse to pulse, allowing accurate measurement of the signal. The transient can be reduced in the case of good quality switching diodes with higher on-off 
current ratio by reducing the capacitance of the switching diodes and the magnitudes of the applied gate biases.

The light signal was determined by subtracting the output pulse height without light illumination from the output pulse height with it in order to eliminate the feedthrough transient effect. Fig. 5.8 shows the measured output signal as a function of the delay time $T_{d}$. The signal drops as the delay time increases. The effective decay time of $17 \mathrm{msec}$ (signal drops to $1 / \mathrm{e}$ ) is equivalent to $\sim 17 \mathrm{G} \Omega$ of reverse bias dynamic resistance since $\mathrm{C}_{\mathrm{d}} \approx$ 1 pF. Fig. 5.9 shows the measured output as a function of integration time. The output signal saturated at $3 \sim 5 \mu \mathrm{sec}$ which agreed well with the discharging time, $\mathrm{T}_{\mathrm{r}}$, of $3.5 \mu \mathrm{sec}$ calculated by the relation

$$
\mathrm{T}_{\mathrm{r}}=\left(\frac{\mathrm{R}_{\mathrm{f}}}{2}\right) \times \mathrm{C}_{\text {det }}=\frac{7 \mathrm{M} \Omega \times 1 \mathrm{pF}}{2}=3.5 \mu \mathrm{sec}
$$

where division by 2 is due to the fact that there are two switching diodes.

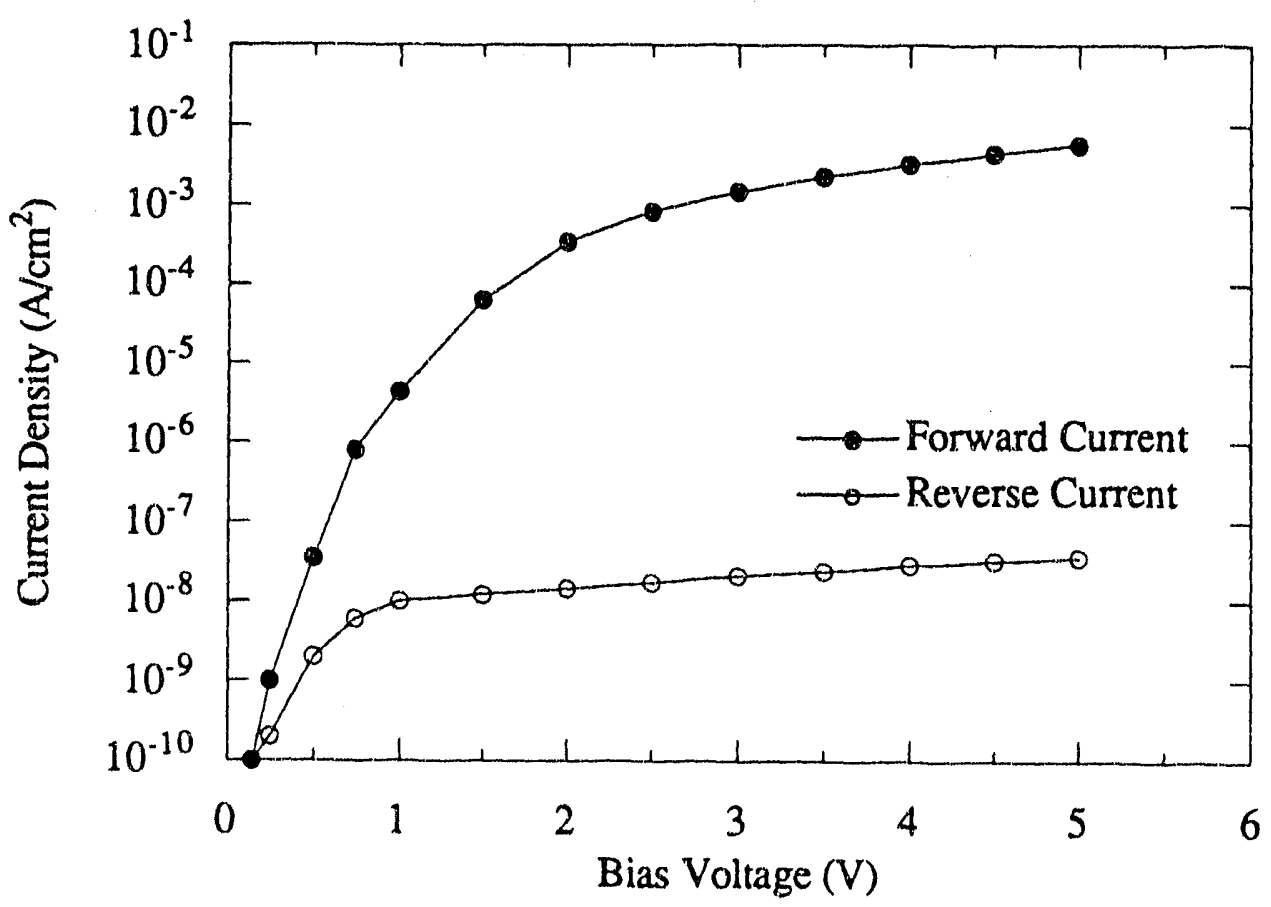

Fig. 5.6 Measurement of forward and reverse current density of $2 \mu \mathrm{m}$ thick pin diode used for switching diodes. 


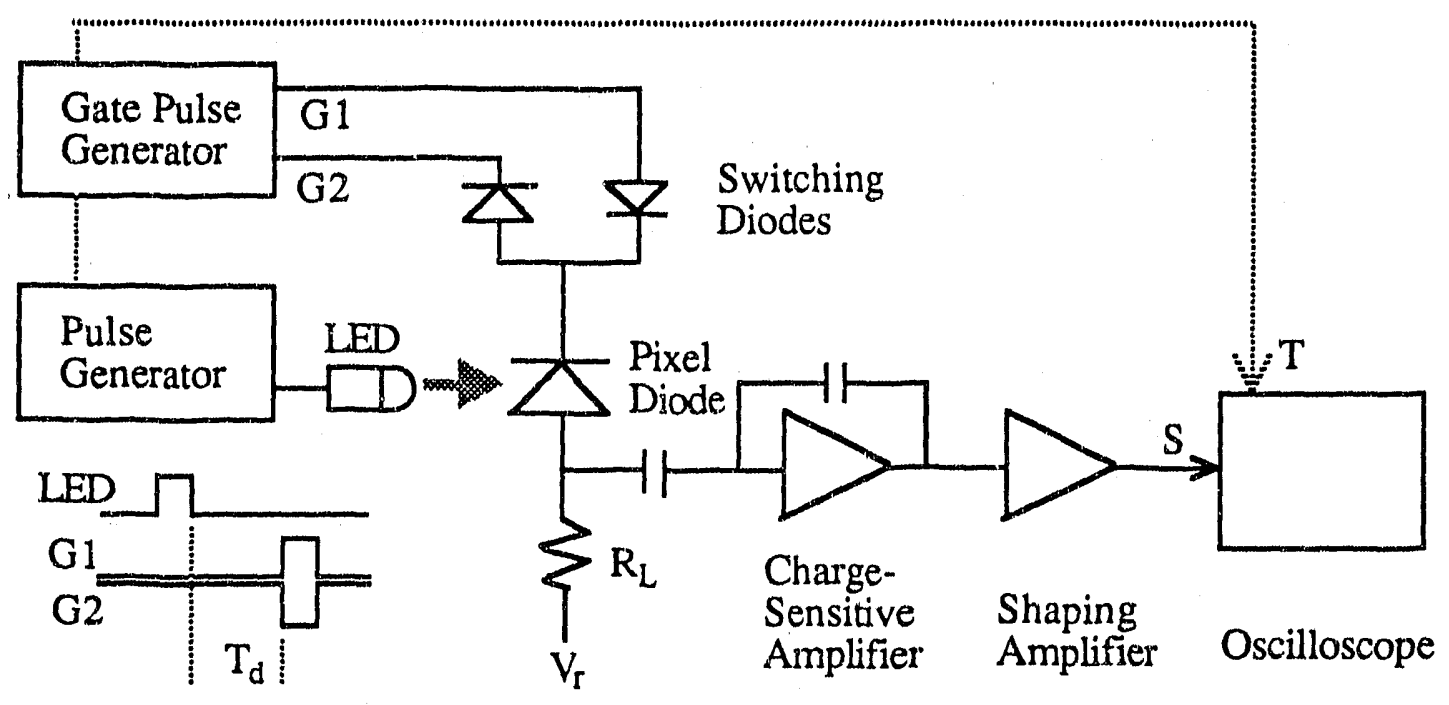

Fig. 5.7 A measurement setup for the signal charge transfer and loss from a pixel diode using a two-diodes switching readout method.

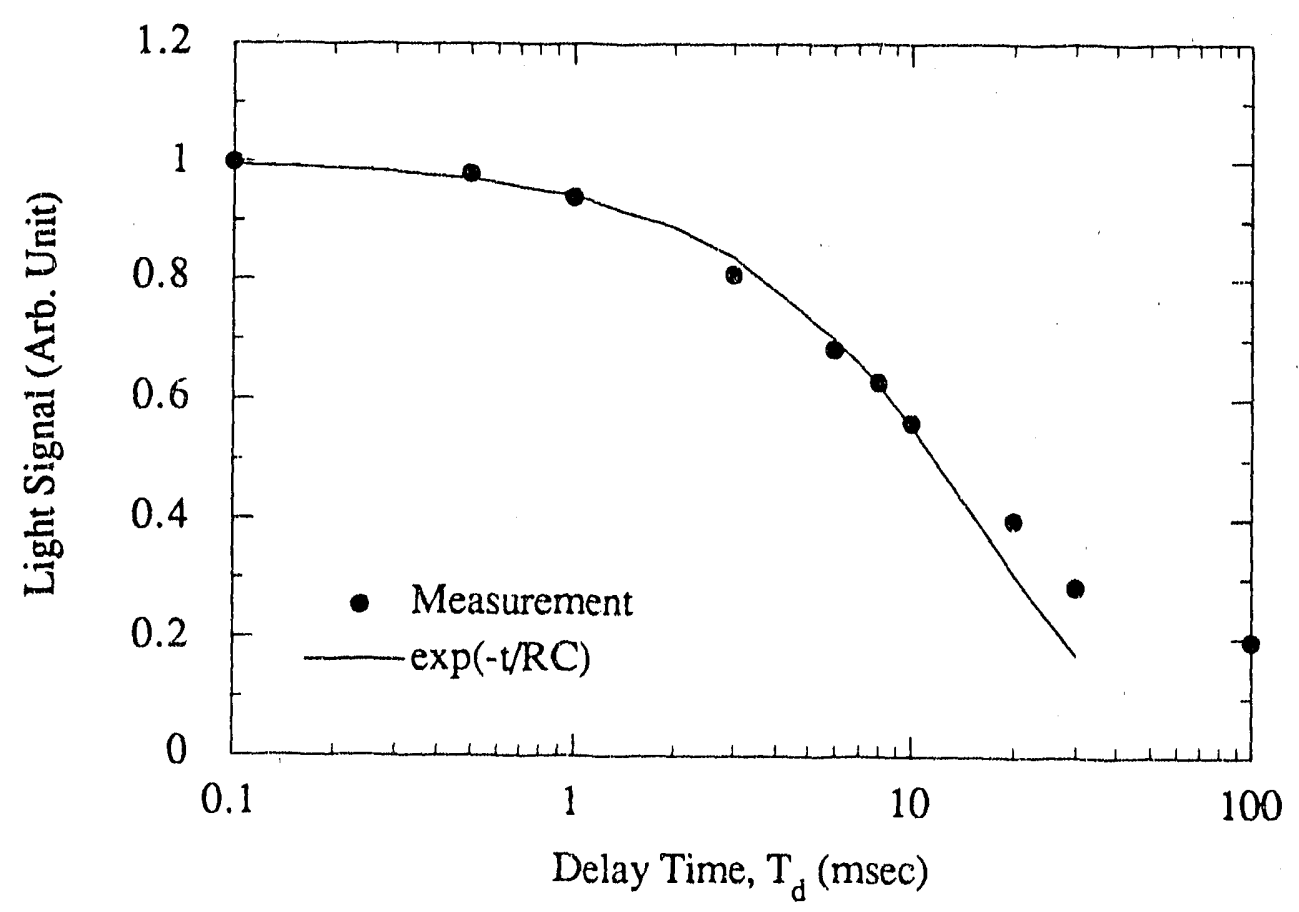

Fig. 5.8 Measurement of light signal as a function of delay time between the time of incidence of light and the time of measurement. (Measured RC time was 17 msec) 


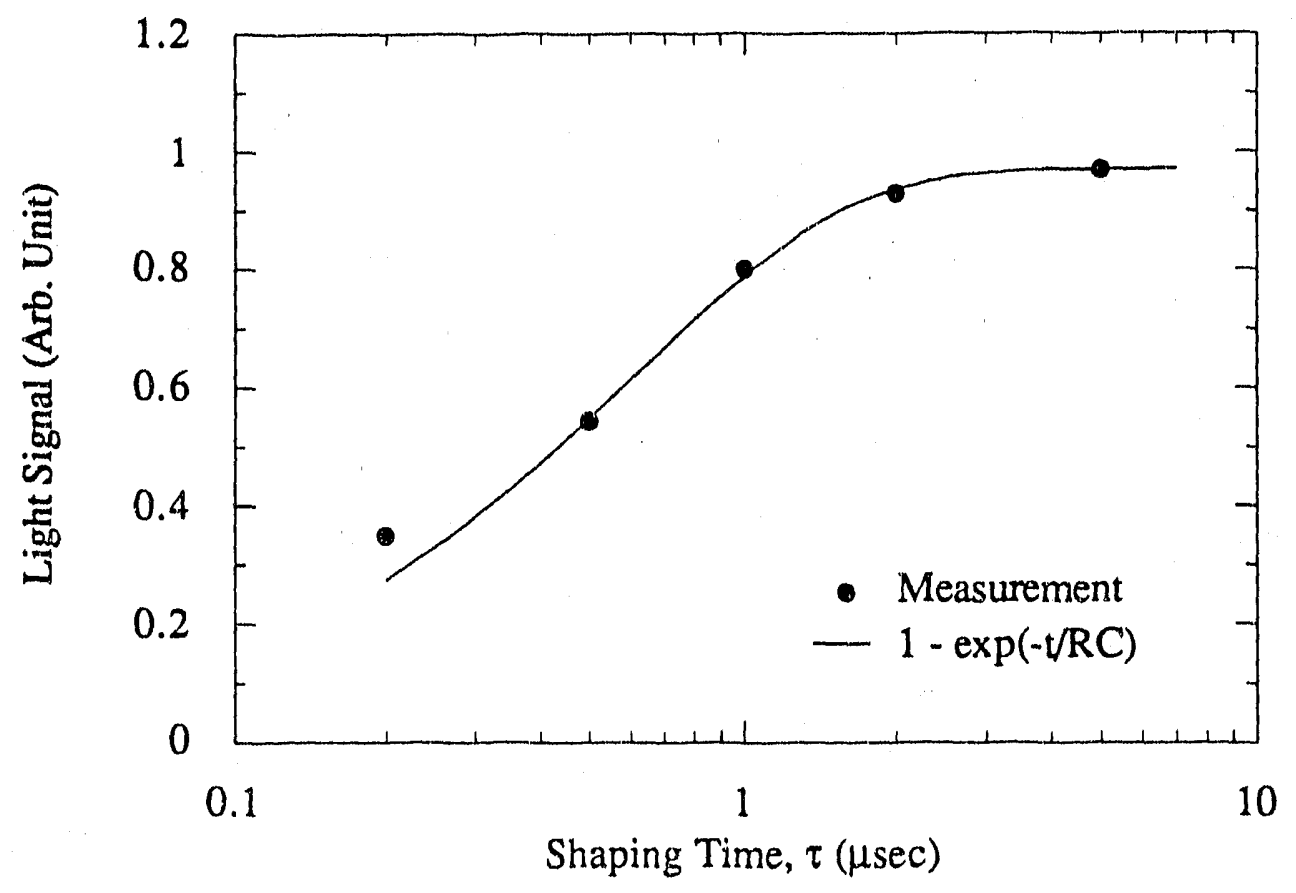

Fig. 5.9 Measurement of light signal as a function of shaping time of CR-RC shaping amplifier. (RC time measured was $0.7 \mu \mathrm{sec}$ )

\subsection{A Poly-Si TFT Pixel Amplifier}

A prototype amplifier circuit was designed, fabricated and tested in order to determine the feasibility of making charge-sensitive pixel amplifiers for a-Si:H pixel detectors using the poly-Si TFT technology developed at Xerox PARC.[5] In actual pixel detectors, in addition to the amplifying stage, other signal processing units such as sampling, holding and readout switch are necessary but they were not implemented in this prototype.

Design requirements for the pixel amplifiers were; (a) small size limited to a pixel area (b) moderate amplifying gain in order to readout the signal through a large-area detector array without picking up extra noise, (c) enough bandwidth to respond to a fast rise of the input current determined by the charge collection in the detector $(\sim \mu \mathrm{sec})$, (d) low 
noise to get a maximum signal-to-noise ratio, (e) low power dissipation, and (f) circuit simplicity for easy fabrication and high reliability.

Large-area poly-Si TFT technology has some limitations in designing an analog amplifier, namely : (a) transconductance $g_{m}$ of a single TFT is limited by the low field effect mobility $\mu_{\mathrm{fe}}$ compared to that of a crystalline MOSFET, (b) minimum feature size is limited by the fabrication process for large-area devices, [6] and (c) no depletion mode TFT is available.

\subsubsection{Design}

The prototype pixel amplifier was designed to consist of three stages; the first stage is a low-noise charge-sensitive amplifier which integrates the signal charge from a detector, the second stage is a voltage amplifier to give an additional gain and the final stage is a source follower output stage with small output impedance to drive the readout lines. The circuit diagram of the test amplifier is shown in Fig. 5.10.

The first charge-sensitive stage consists of an inverting voltage amplifier and a feedback capacitor.

Two important design concepts for the first stage were; (a) for the maximum openloop gain the channel length $L$ of the front-end TFT was chosen to be the minimum feature size $(5 \mu \mathrm{m})$ and for minimum noise the channel width $\mathrm{W}(50 \mu \mathrm{m})$ was chosen to make the input capacitunce of the charge-sensitive amplifier equal to that of an arbitrary pixel detector, $0.2 \mathrm{pF}$. This value is equivalent to the capacitance of an a-Si:H pixel of area 300 $\mu \mathrm{m} \times 300 \mu \mathrm{m}$ and thickness $50 \mu \mathrm{m}$.(b) for the maximum closed-loop charge gain the feedback capacitance must be minimized. The capacitor was made using the same dielectric used as a gate insulator of TFTs, which was $100 \mathrm{~nm}$ thick $\mathrm{SiO}_{2}$. We took, arbitrarily, as the capacitor's dimensions, twice the minimum feature size, or $10 \mu \mathrm{m}$, which gave a $\mathrm{CF}_{\mathrm{F}} \approx$ $0.02 \mathrm{pF}$. Therefore the effective voltage gain, the ratio of the detector capacitance to the feedback capacitance, would be $\sim 10$ when the open-loop voltage gain is large enough. 
The voltage amplifier is implemented by an $n$-channel single-ended common-source poly-Si TFT N1, cascoded with a TFT N2, and a current-source load.[7] Although pchannel poly-Si TFTs have lower noise, an n-channel TFT was selected as the front-end because its threshold voltage is smaller and more stable than that of p-channel TFTs.

A cascoded configuration was used to minimize the Miller effect which causes the gate-to-drain capacitance (overlapping) to be effectively increased by the gain factor, thereby reducing the bandwidth. The load was designed as a p-channel TFT current source. This complementary configuration was expected to give a higher gain and more reliability than a resistive load or $\mathrm{n}$-channel TFT load. Thin-film resistors such as $\mathrm{n}$ - or $\mathrm{p}$-doped polySi layers are unstable with time. [8]

Finally another n-channel TFT was connected in parallel with the feedback capacitor for biasing the input node and reset the amplifier by discharging the feedback capacitor. The second stage is an extra voltage-gain stage which amplifies the signal further so that the amplified signal pulse will reach the external circuit with minimal addition of perturbation by the extra noise sources such as inter-communication, pick-up noise, etc. The impedances of the bus line and the external circuit were not considered here, but will be considered for a more complete design.

The final stage is simply a source follower stage, which has unity gain and low output impedance, to drive the external load without distortion of the output pulse shape.

Except for the front-end TFT, the dimensions of the other TFTs and operation biases required by the circuit is shown in Fig. 5.10 were determined using a circuit simulation program, PSPICE* [9] in order to achieve (a) a moderate gain of the second stage ( $\sim 10)$, (b) a maximum gain-bandwidth, and (c) a minimum power dissipation. In the program, a simple crystal-Si MOSFET model was used. For example, the drain-to-source current in the saturation region is given by the following Shichman and Hodges model.[10]

\footnotetext{
* PSPICE is a commercial version of SPICE developed at MicroSym. Corp. Palo Alto.
} 


$$
\mathrm{I}_{\mathrm{ds}}=\frac{\mathrm{W}}{\mathrm{L}} \frac{\mathrm{K}_{\mathrm{p}}}{2}\left(1+\lambda \mathrm{V}_{\mathrm{ds}}\right)\left(\mathrm{V}_{\mathrm{gs}}-\mathrm{V}_{\mathrm{T}}\right)^{2}
$$

where $\lambda$ is the channel-length modulation coefficient, $K_{p}=\mu_{\mathrm{fe}} \varepsilon_{0} \varepsilon_{\mathrm{i}} / \mathrm{t}_{\mathrm{i}}$ and other symbols are the same as defined in Chapter 4 . The oxide thickness, $t_{i}$ is $100 \mathrm{~nm}$ which is a typical value for the siandard poly-Si TFT process at Xerox PARC.[5] Table 5.2 shows the input parameter's used in PSPICE for the n-channel and p-channel poly-Si TFTs. Data were taken from reference [11]. Table 5.3 shows the resultant dimensions of TFTs and capacitors.

Table 5.2 Input parameters of n-channel and p-channel poly-Si TFT for PSPICE

\begin{tabular}{|c||c|c|c|c|c|c|}
\hline Type & $\mathrm{L}(\mu \mathrm{m})$ & $\mathrm{K}_{\mathrm{p}}\left(\mu \mathrm{A} / \mathrm{V}^{2}\right)$ & $\mathrm{V}_{\mathrm{T}}(\mathrm{V})$ & $\lambda\left(\mathrm{V}^{-1}\right)$ & $\mathrm{t}_{\mathrm{i}}(\mathrm{nm})$ & $\mathrm{C}_{\mathrm{OV}}{ }^{*}(\mathrm{pF} / \mu \mathrm{m})$ \\
\hline \hline \multirow{3}{*}{ n-channel } & 5 & 0.8 & 2.5 & 0.07 & 100 & 0.02 \\
& 10 & 1.5 & 2.5 & 0.033 & 100 & 0.02 \\
& 50 & 2.4 & 2.5 & 0.02 & 100 & 0.02 \\
\hline p-channel & 15 & 0.4 & -2.5 & 0.01 & 100 & 0.02 \\
\hline
\end{tabular}

* $\mathrm{C}_{\mathrm{ov}}$ is the gate-drain or gate-source overlapping capacitance per unit gate width. 


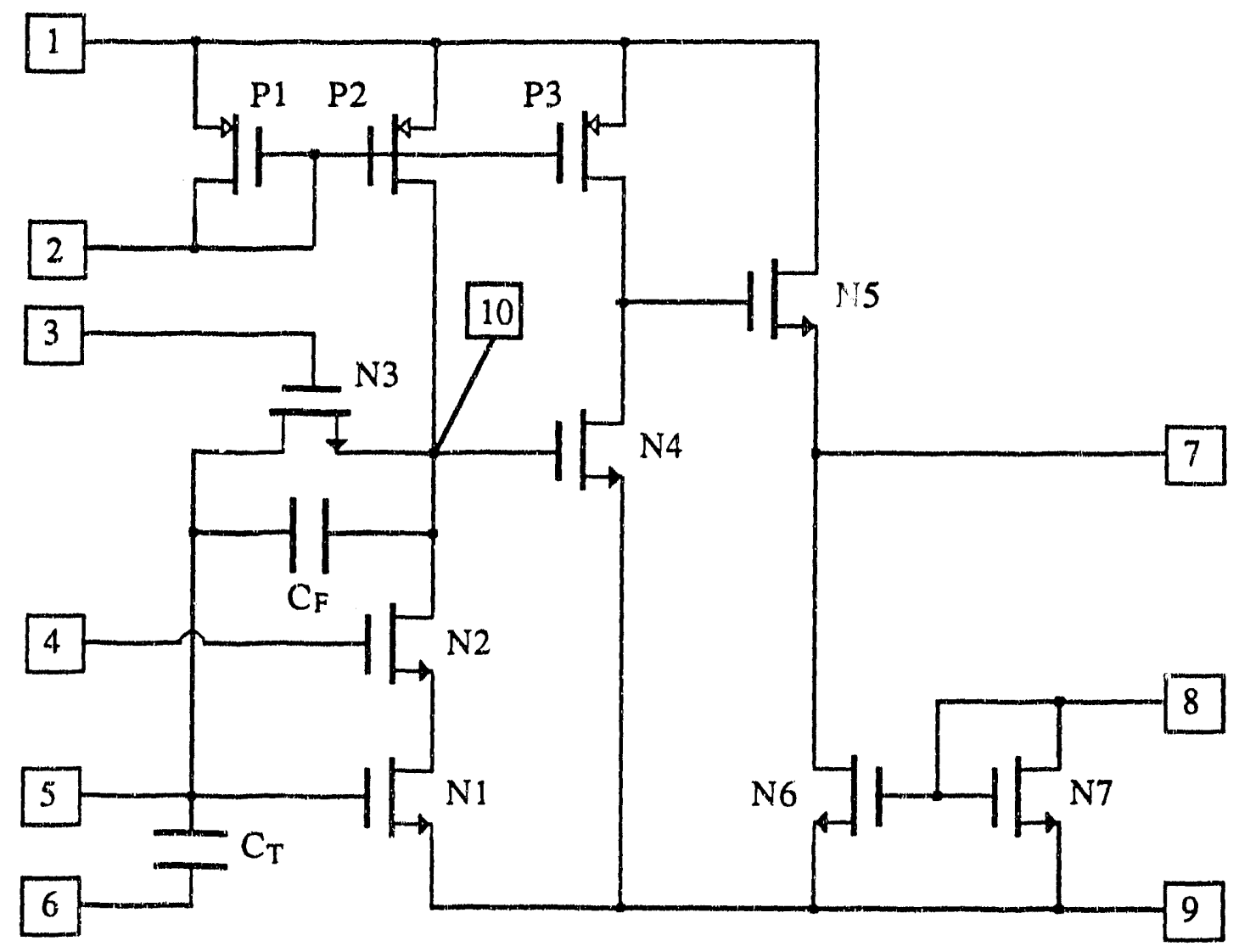

Fig. 5.10 The schematic circuit diagram of the prototype poly-Si CMOS charge-sensitive pixel amplifiers for a-Si:H pixel detectors. Each square with node numbers represents a test pad. $\mathrm{N}$ and $\mathrm{P}$ stand for n'channel and p-channel TFTs, and $\mathrm{C}$ stands for capacitors.

Table 5.3 A list of components and their dimensions

Table 5.4 Nodes

$\begin{array}{lll}\text { N1 } & \text { Front-end TFT } & \text { W/L }=50 / 5 \mu \mathrm{m} \\ \text { N2 } & \text { Cascode TFT } & W / L=30 / 5 \mu \mathrm{m} \\ \text { N3 } & \text { Reset switch TFT } & W / L=10 / 10 \mu \mathrm{m} \\ \text { N4 } & \text { Second stage TFT } & \mathrm{W} / \mathrm{L}=50 / 10 \mu \mathrm{m} \\ \text { N5 } & \text { Source follower TFT } & \mathrm{W} / \mathrm{L}=10 / 50 \mu \mathrm{m} \\ \text { N6,7 } & \text { Load of N5 } & \mathrm{W} / \mathrm{L}=10 / 50 \mu \mathrm{m} \\ \text { P1 3 } & \text { Current source load } & \mathrm{W} / \mathrm{L}=20 / 15 \mu \mathrm{m} \\ \text { CT }_{\mathrm{T}} & \text { Test capacitor } & \text { Area }=10 \times 10 \mu \mathrm{m} \\ \text { CF } & \text { Feedback capacitor } & \text { Area }=10 \times 10 \mu \mathrm{m}\end{array}$

1 Bias $V_{d d}$

2 Bias $V_{p}$

3 Reset pulse

4 Bias $V_{c}$

5 Input

6 Test pulse

7 Output

8 Bias $V_{n}$

9 Ground

10 Output of stage 1 


\subsubsection{Fabrication}

In order to test each stage, as well as the complete circuit, individually, several combinations of test amplifiers were fabricated; first stage only, second stage only, and the complete amplifier with and without the cascode configuration. The complete amplifier, including interconnection lines occupied an area of $200 \mu \mathrm{m} \times 100 \mu \mathrm{m}$ which is well within the target pixel of $300 \mu \mathrm{m} \times 300 \mu \mathrm{m}$. On the test chip, we also made individual TFTs having the same dimensions as TFTs used in the prototype amplifier to check the input parameters used in the design. The interconnections and test pads were drawn for convenience and easy identification of components. The test circuit was fabricated using the standard low temperature poly-Si TFTs process at Xerox PARC. The process is described in reference [5].

\subsubsection{Test and Results}

\section{(1) Individual_TFTs}

Before the circuit was tested, the individual TFTs were separately tested in order to naake sure the fabrication process was satisfactory, and to find the range of operating bias voltages for each node.

Fig. $5.11 \sim$ Fig. 5.14 show the dc characteristics of the n-channel and p-channel poly-Si TFTs. As seen in the figures, the TFTs show an I-V curve similar to that of crystalSi MOSFET in the low bias region (dotted line). In the high bias region, however, the short-channel effect makes a kink in the current a little earlier than in the crystal MOSFET. The data are also compared to a poly-Si TFT model (solid lines) developed by Byun et al. at University of Virginia and Xerox PARC. [12] For these n-channel poly-Si TFTs, the onset voltage $\mathrm{V}_{\mathrm{S}}$ of the short channel effect was at a drain-to-source voltage of $5 \mathrm{~V}$ for $\mathrm{L}=$ $5 \mu \mathrm{m}$ and $6 \mathrm{~V}$ for $\mathrm{L}=10 \mu \mathrm{mi}$. For $\mathrm{p}$-channel poly-Si TFTs, $\mathrm{V}_{\mathrm{s}}$ was $9 \mathrm{~V}$ for $\mathrm{L}=15 \mu \mathrm{m}$. Table 5.5 shows the measured values of some characteristics of individual poly-Si TFTs. 


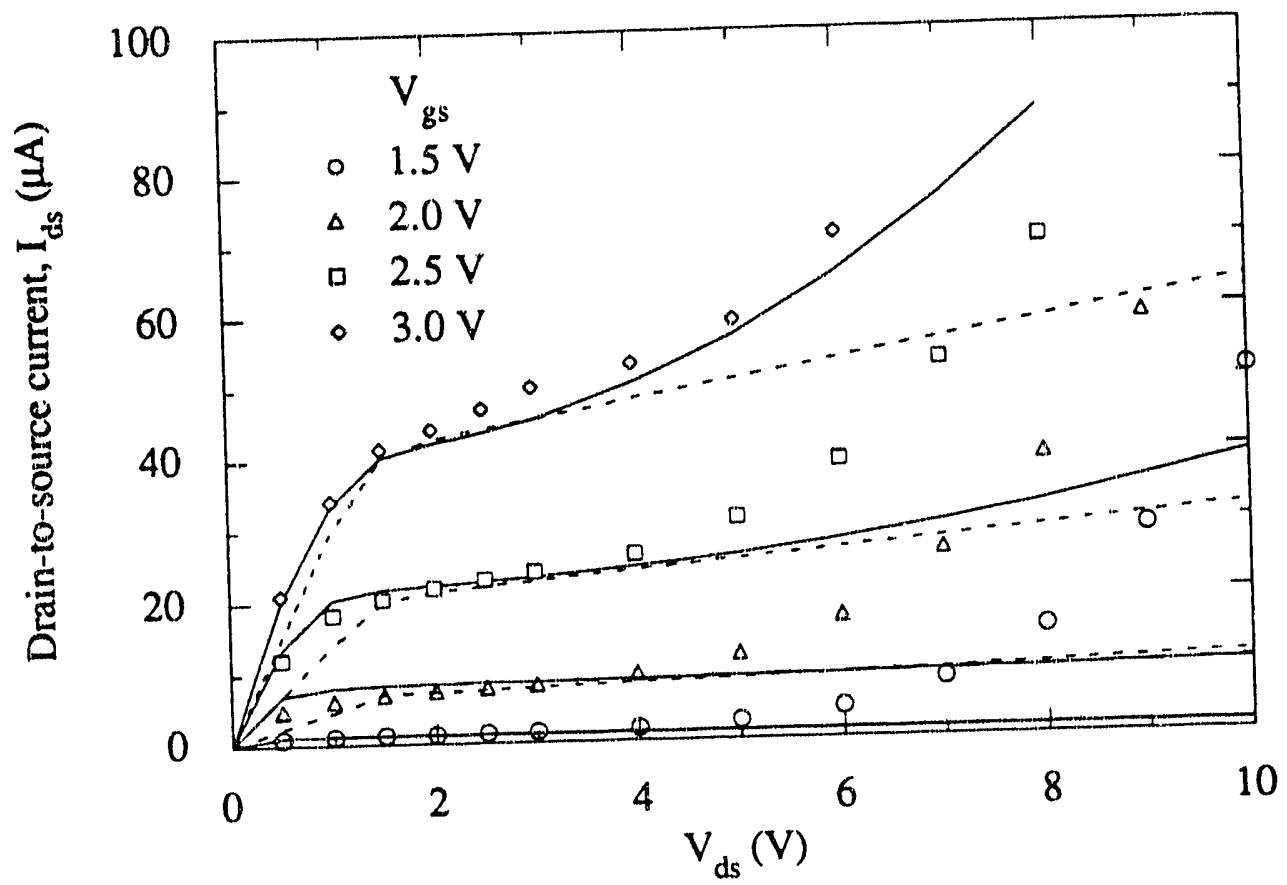

Fig. 5.11 I-V Characteristics of n-channel poly-Si TFT. $W / L=50 / 5 \mu \mathrm{m}$.

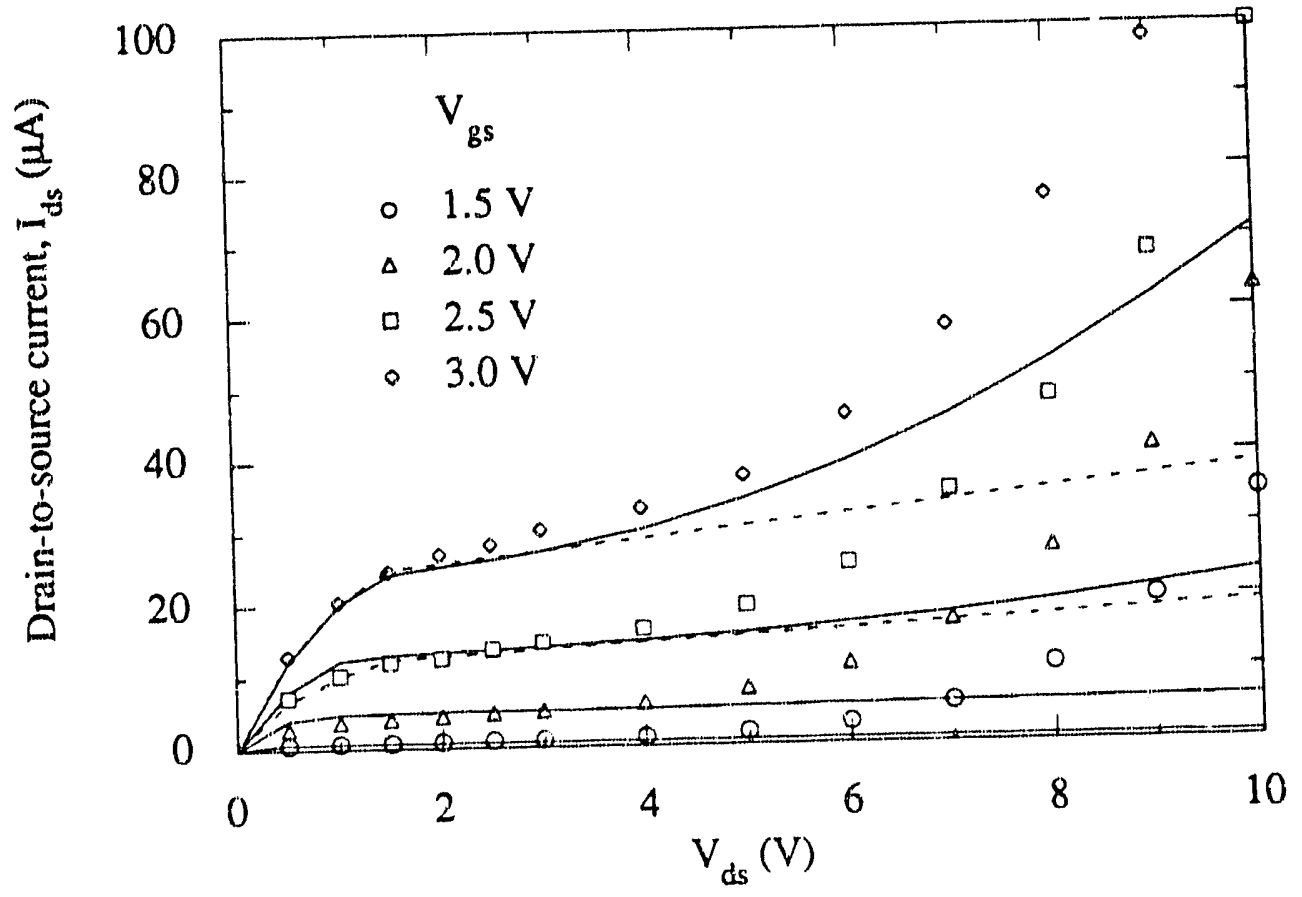

Fig. $5.12 \mathrm{I}-\mathrm{V}$ Characteristics of $\mathrm{n}$-channel poly-Si TFT. W/L=30/5 $\mu \mathrm{m}$. 


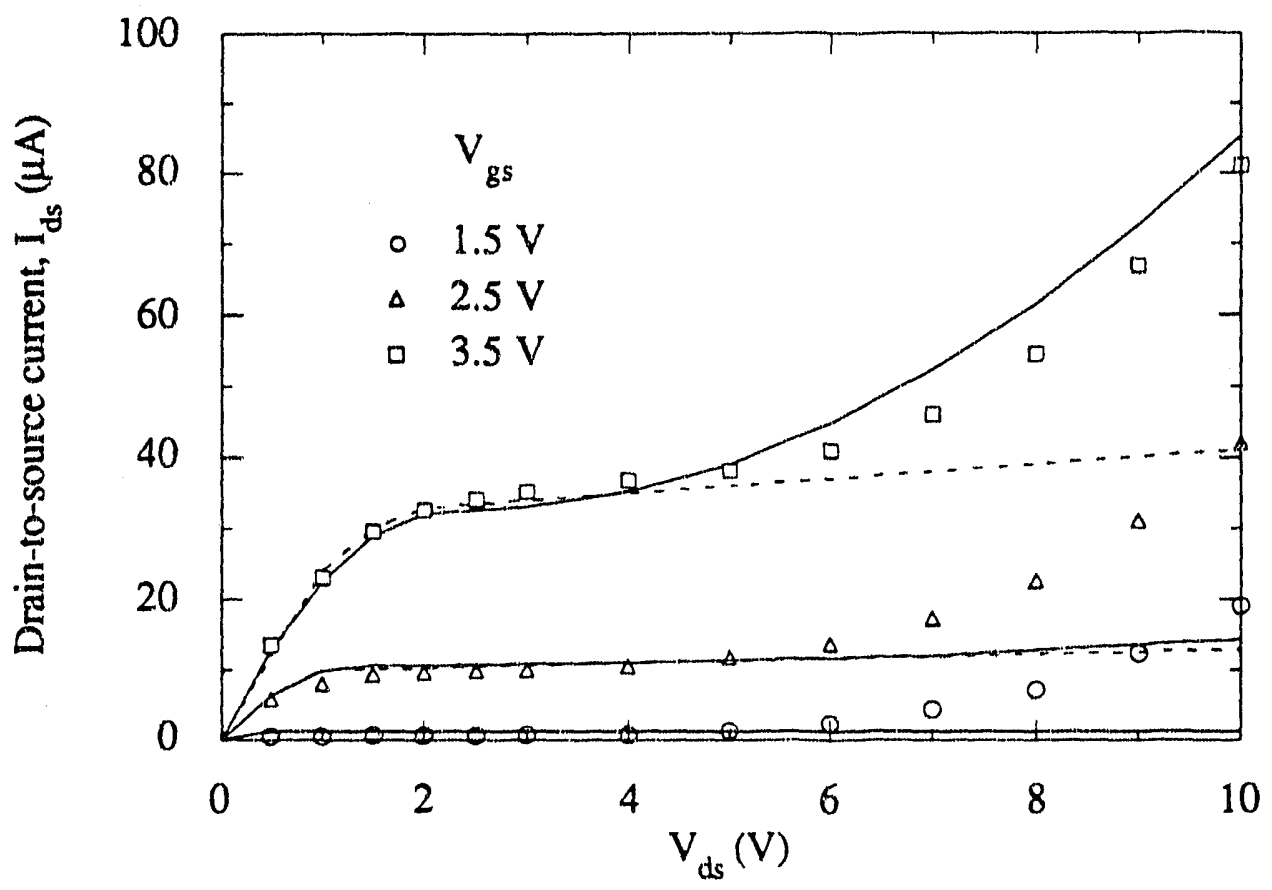

Fig. 5.13 I-V Characteristics of n-channel poly-Si TFT. W/L=50/10 $\mu \mathrm{m}$.

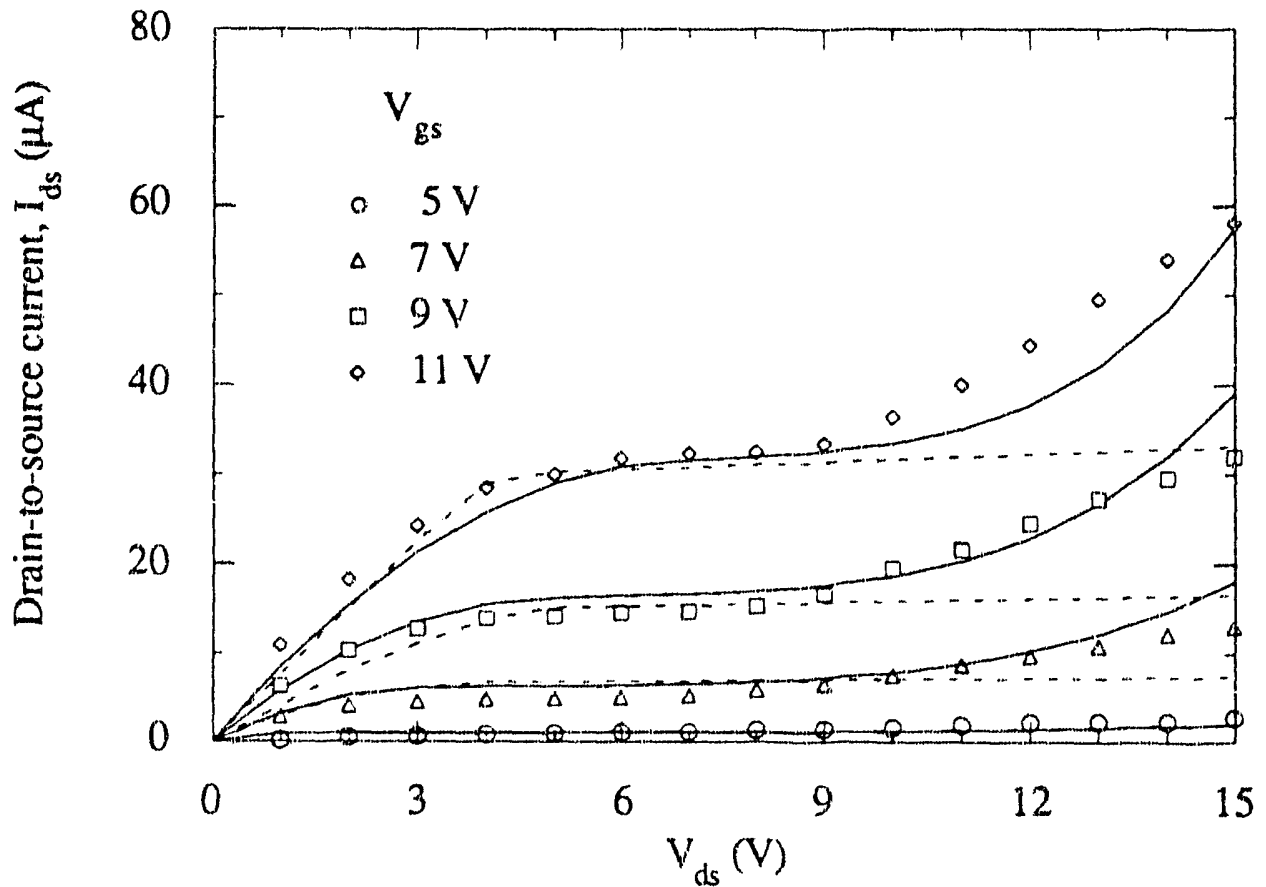

Fig. 5.14 I-V Characteristics of p-channel poly-Si TFT. W/L $=20 / 15 \mu \mathrm{m}$. 
Table 5.5 Measured circuit parameters of poly-Si TFTs

\begin{tabular}{|c||c|c|c|c|c|}
\hline & $\mathrm{L}(\mu \mathrm{m})$ & $\mathrm{K}_{\mathrm{p}}\left(\mu \mathrm{A} / \mathrm{N}^{2}\right)$ & $\mu_{\mathrm{fe}}\left(\mathrm{cm}^{2} / \mathrm{Vsec}\right)$ & $\mathrm{V}_{\mathrm{T}}(\mathrm{V})$ & $\mathrm{V}_{\mathrm{S}}(\mathrm{V})$ \\
\hline $\mathrm{n}$-ch TFT & 5 & 2.6 & 73 & 1.3 & 5 \\
\hline $\mathrm{n}$-ch TFT & 10 & 3.2 & 90 & $\vdots .4$ & 6 \\
\hline p-ch TFT & 15 & 0.6 & 17 & 3.0 & 9 \\
\hline
\end{tabular}

\section{(2) Front-End Charge-Sensitive Amplifier Stage}

Because of the short channel effect, which was not considered at the time of design and the difference between the design and measured values of the circuit parameters such as the threshold voltage, the input stage gate bias was supplied separately with a probe during the test instead of through the reset TFr N3.

The measured dc voltage swing of the first stage with and without the cascode is plotted in Fig. 5.15. The dc gain $A_{0}$ which is the slope of the curves shown in the figure is $\sim 11$ and $\sim 8$ respectively with and without the cascode. The cascode configuration gives better linearity in the dc gain. The closed-loop gain, $A_{c}$, of the charge-sensitive amplifier would be reduced to $\sim 7$ and $\sim 5.5$ respectively when it is connected to a detector capacitance $C_{d}$ of $0.2 \mathrm{pF}$. (See the equation (3.7) in Chapter 3.) The bias conditions are given in the figure caption.

To find the frequency response, a sinusoidal wave with a magnitude $10 \mathrm{mV}$ was introduced to the input node through an external coupling capacitor $(\sim 0.01 \mu \mathrm{F})$, and the output wave form was measured at the output node of the first stage (node 10 in Fig. 5.10). Fig. 5.16 shows the first stage gain with and without the cascode. The measured $3 \mathrm{~dB}$ cut-off frequency was found to be about 1 and $0.8 \mathrm{MHz}$ in to the oscilloscope probe load $(1 \mathrm{pF}+1 \mathrm{M} \Omega)$ from the equation for the ac gain $\mathrm{A}(\mathrm{f})$ given by

$$
A(f)=\frac{A_{0}}{\sqrt{1+\left(f / f_{3 d B}\right)^{2}}}
$$




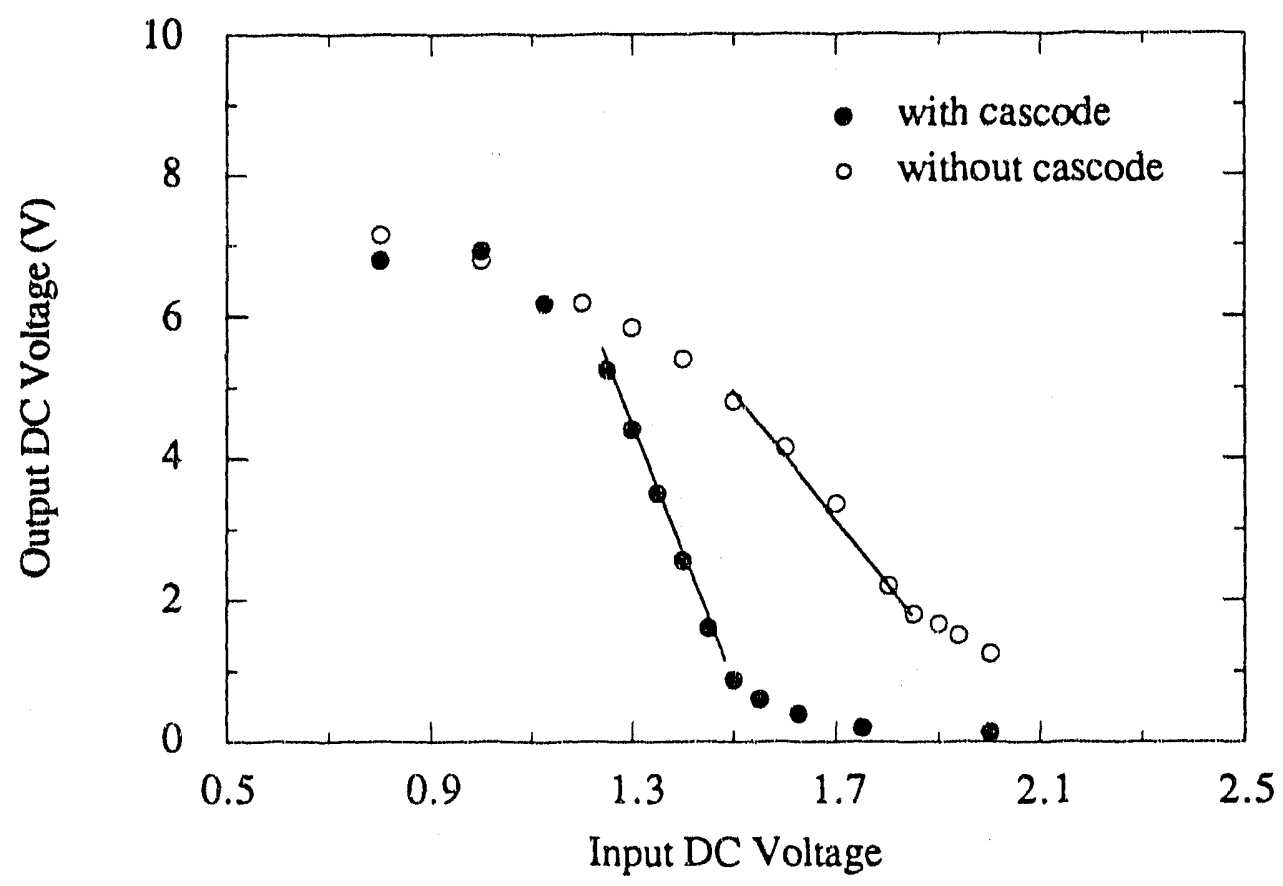

Fig. 5.15 DC characteristics of the first stage with and without cascode configuration. $V_{d d}$ $=10 \mathrm{~V}, \mathrm{~V}_{\mathrm{p}}=5 \mathrm{~V}$ and $\mathrm{V}_{\mathrm{c}}=5 \mathrm{~V}$.

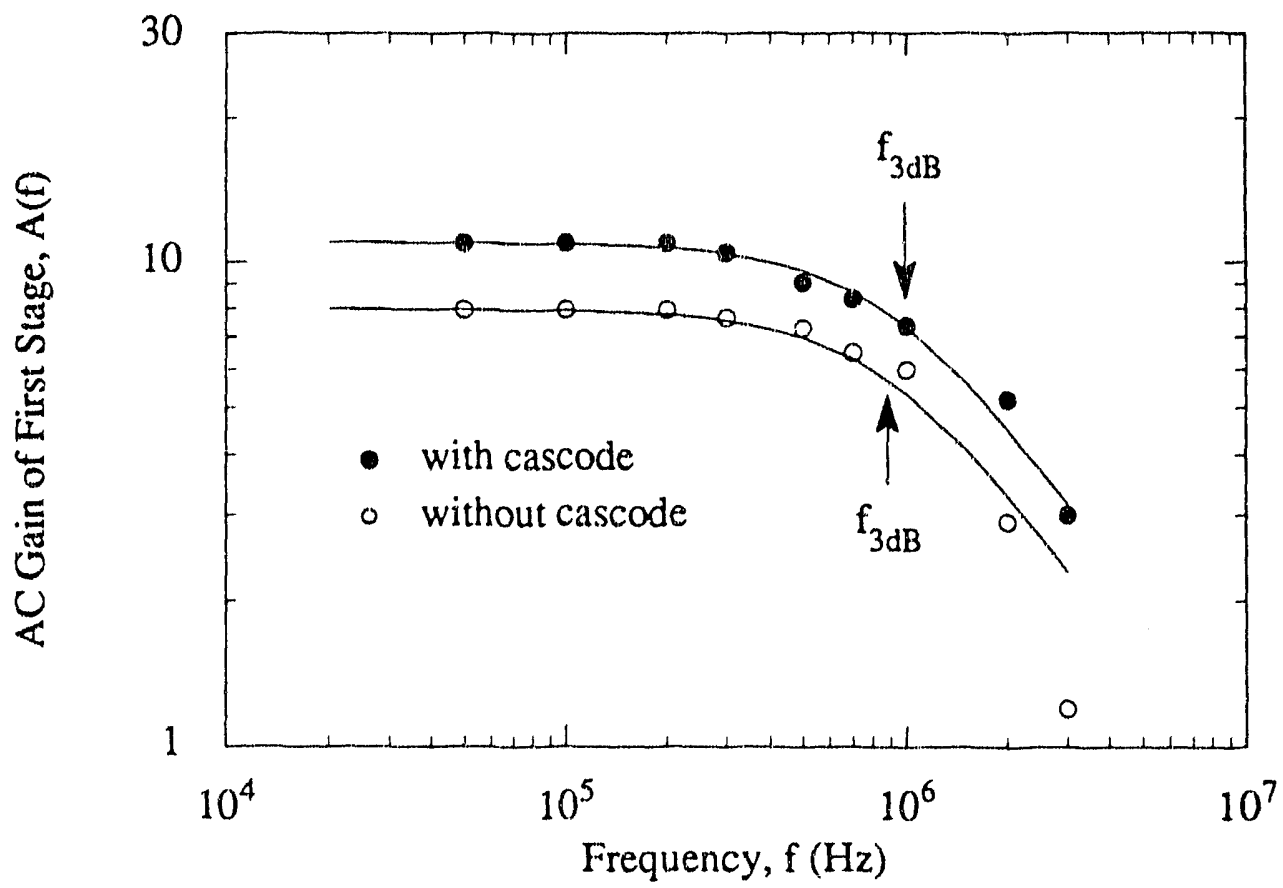

Fig. 5.16 Gain and frequency curves of the first stage with and without cascode configuration. $V_{d d}=10 \mathrm{~V}, V_{p}=5 \mathrm{~V}$ and $V_{c}=5 \mathrm{~V}$. 
The measured values are equivalent to 3.5 and $2.8 \mathrm{MHz}$ after subtraction of the loading effect of the probe assuming

$$
f_{3 d B}=f_{m 3 d B} \times\left(C_{N 3}+C_{\text {probe }}\right) / C_{N 3}
$$

where $C_{\text {probe }}$ and $C_{\mathrm{N} 3}$ are the load capacitance of the probe $(1 \mathrm{pF})$ and the input capacitance of the next stage $(\sim 0.5 \mathrm{pF})$ respectively.

The overall noise of the amplifier system is governed mainly by the front-end TFT. The frequency spectrum of noise from the same-sized n-channel TFTs as the front-end TFT at the same operation bias was measured using the set-up described in Chapter 4, and is shown in Fig. 5.17. 1/f noise was found to be the dominant noise source up to the measured range of $0.1 \mathrm{MHz}$. Thermal noise was calculated using equation (4.21) with $\theta=$ $2 / 3$ and the measured $g_{m}$ value at the same bias condition. Assuming a CR-(RC) ${ }^{n}$ shaping network ( $n=1$ and $n=4)$, the equivalent input noise charge from the front-end TFT, a sum of $1 / \mathrm{f}$ noise and the channel resistance noise, was estimated as a function of a shaping time using the equations in Table 4.2 in Chapter 4 and is shown in Fig. 5.18. For the range of shaping time $>0.1 \mu \mathrm{sec}, 1 / \mathrm{f}$ noise is dominant and corresponds to $\sim 1040$ and $\sim 970$ electrons rms for cases of $n=1$ and $n=4$ respectively. 


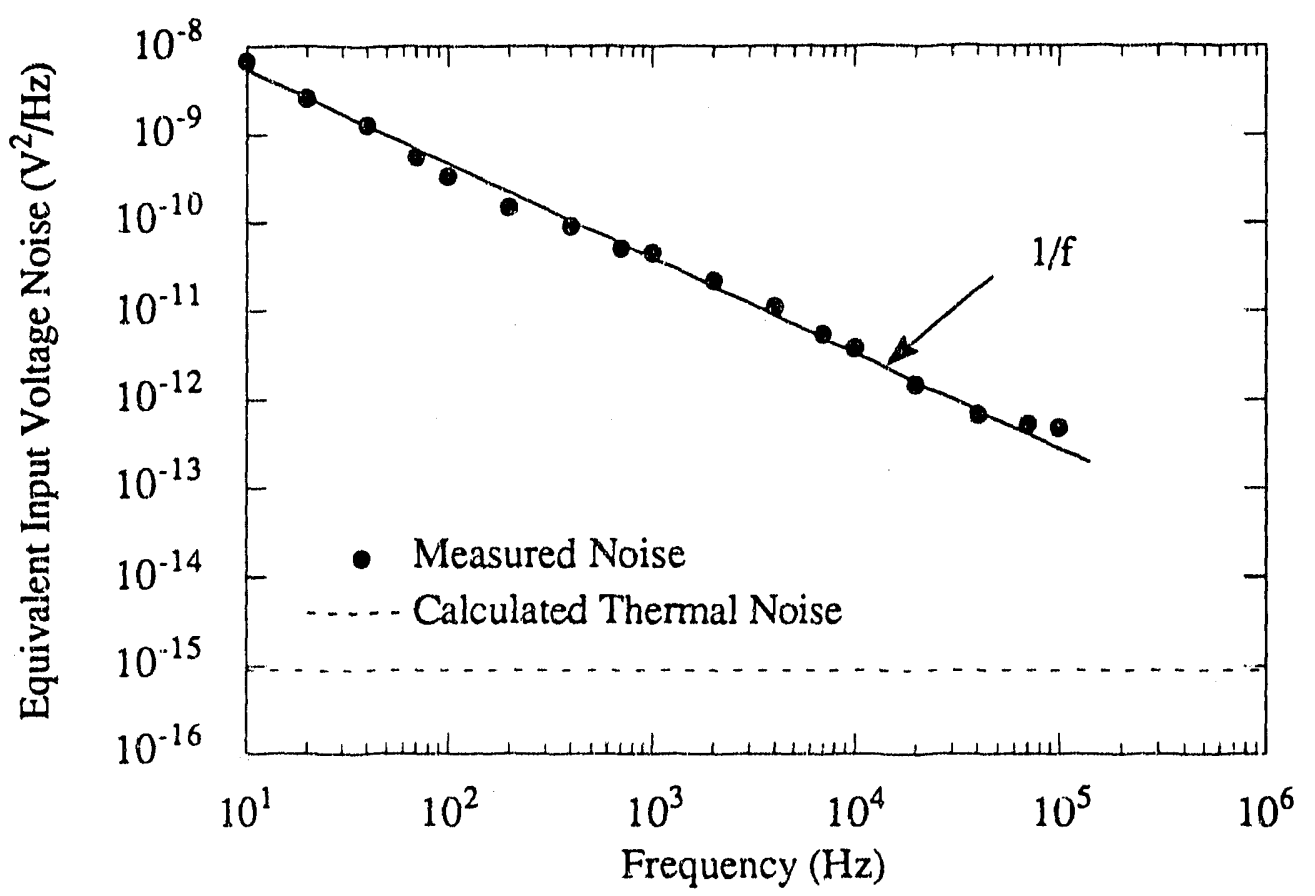

Fig. 5.17 Measured noise power spectrum of the front-end n-channel poly-Si TFT. W/L $=50 / 5 \mu \mathrm{m}$ at $\mathrm{V}_{\mathrm{gs}}=1.8 \mathrm{~V}, \mathrm{~V}_{\mathrm{ds}}=4 \mathrm{~V}$. (operation bias level). $\mathrm{g}_{\mathrm{m}}=\sim 20 \mu \mathrm{A} / \mathrm{V}$.

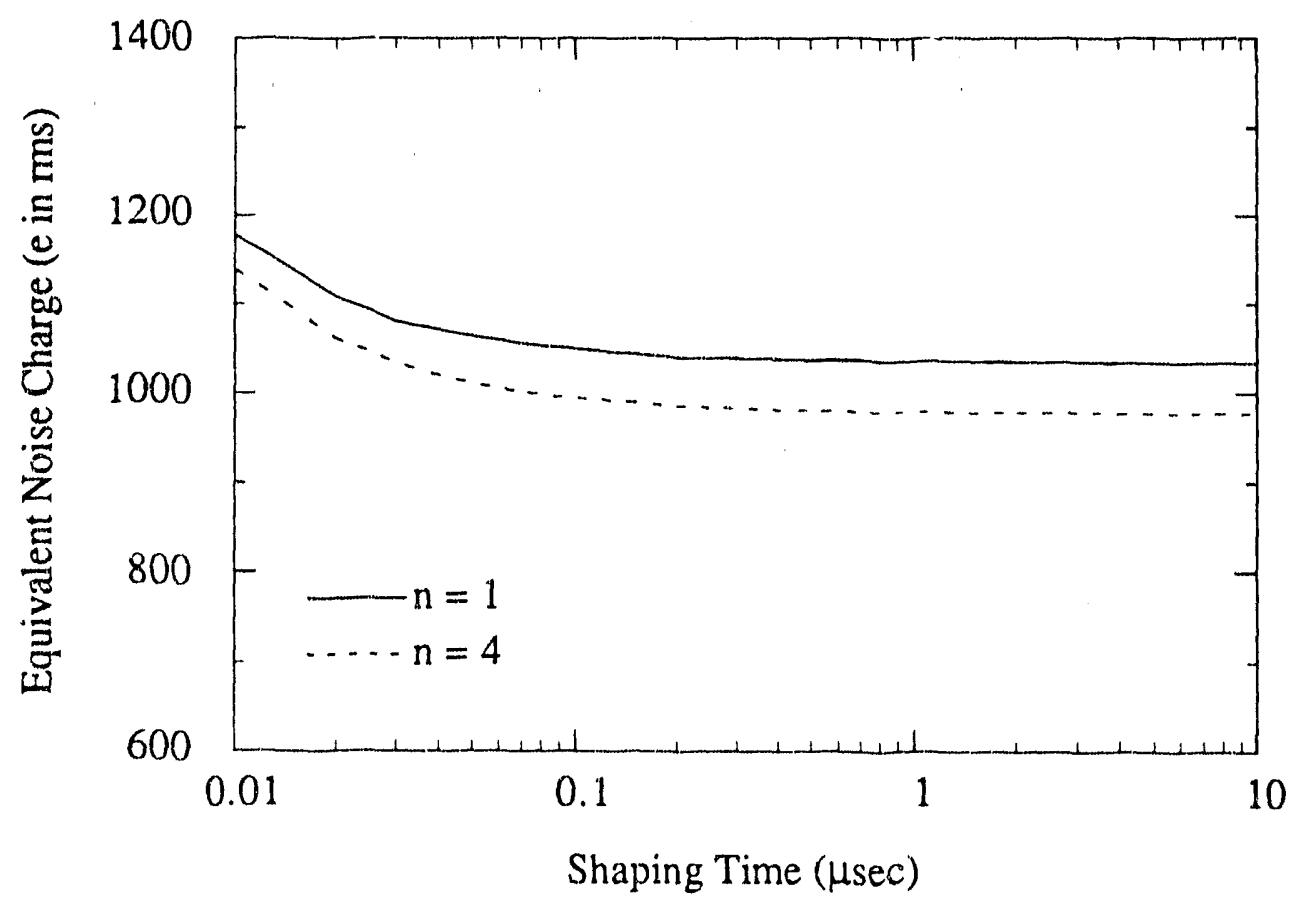

Fig. 5.18 Calculated equivalent noise charge of the front-end $n$-channel poly-Si TFT as a function of the shaping time for $C R-(R C)^{n}$ shaping amplifier with $n=1$ and $n=$ 4. $\mathrm{W} / \mathrm{L}=50 / 5 \mu \mathrm{m}$ and $\mathrm{V}_{\mathrm{gs}}=1.8 \mathrm{~V}, \mathrm{~V}_{\mathrm{ds}}=4 \mathrm{~V}$. (operation bias level). $\mathrm{g}_{\mathrm{m}}=\sim$ $20 \mu \mathrm{A} / \mathrm{V}$. 


\section{(3) Qverall Gain and Band width}

The measured gain of the second stage was about 10 and the third stage was about 1. Fig. 5.19 shows the dc output voltage swing of the second stage as a function of the input voltage swing of the first stage. The measured frequency responses for the complete amplifier is shown in Fig. 5.20 and are 1 and $0.5 \mathrm{MHz}$, respectively with and without the cascode. The estimated $3 \mathrm{~dB}$ cut-off frequencies after calibration of the loading effect of the probe are 3.5 and $1.75 \mathrm{MHz}$ respectively with and without the cascode by a similar relation used previously. Therefore from the relation, $f_{3 \mathrm{~dB}} \times T_{\text {rise }} \approx 0.35,[13]$ the pulse rise times are 100 and $200 \mathrm{nsec}$ with and without the cascode.

The dynamic range of the amplifier is determined by the noise level, estimated previously for the case of $C R-(R C)^{n}$ shaping amplifier, and the range of input voltage swing before the saturation of the output voltage. The lower limit is arbitrarily defined as the total noise in rms from the detector and the amplifier system and is $\sim 1000$ electrons.

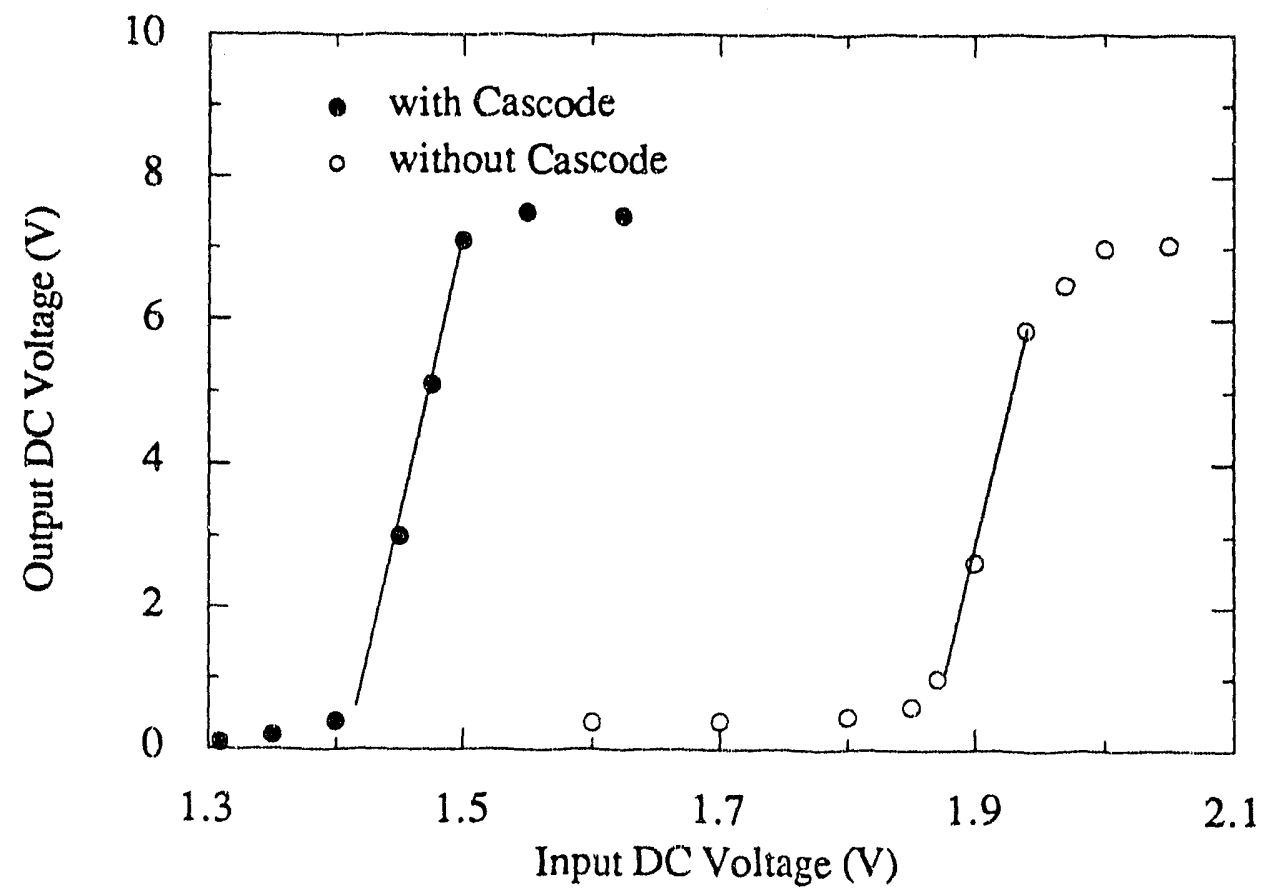

Fig. 5.19 DC characteristics of 2nd stage with and without the cascode configuration. 


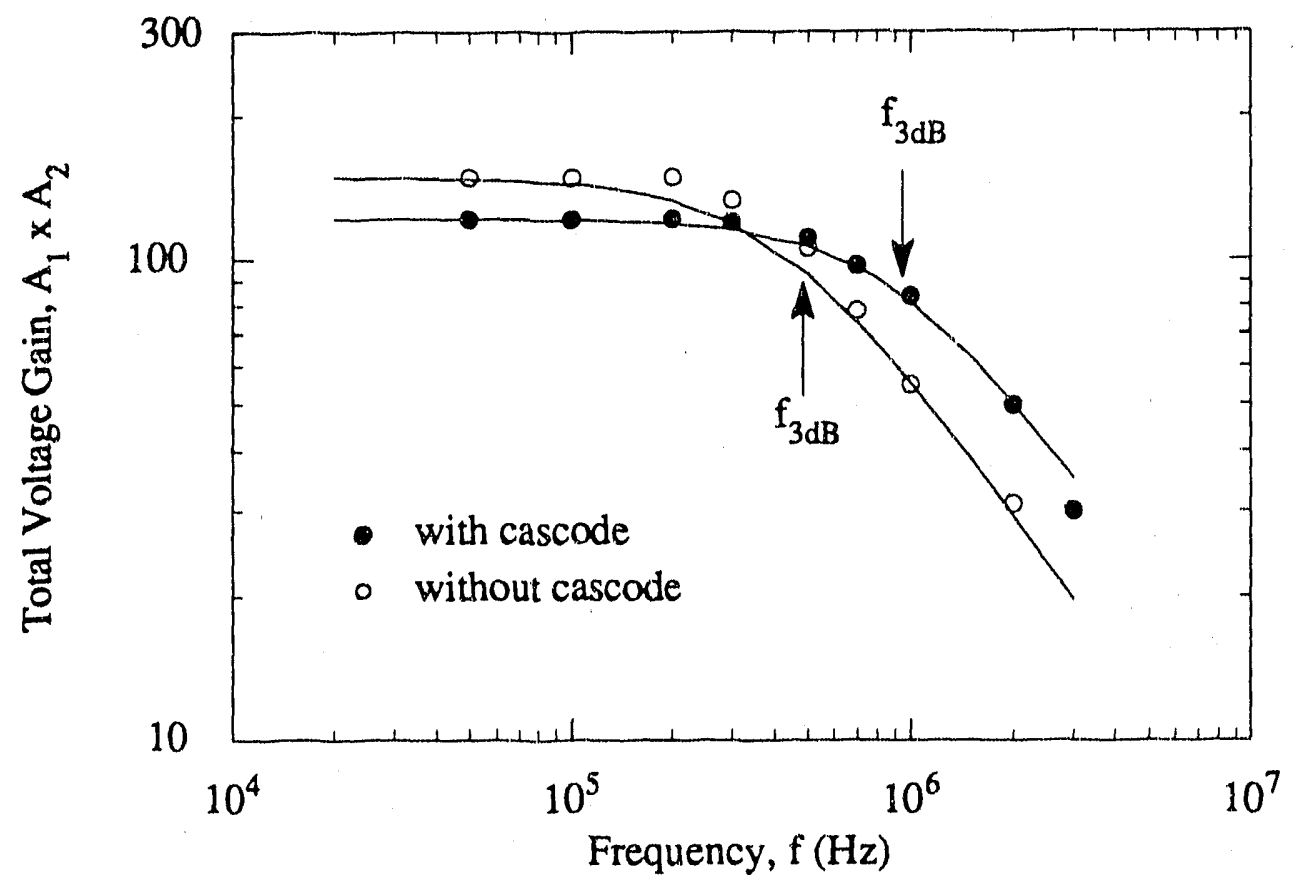

Fig. 5.20 Total voltage gain of the complete amplifier with and without the cascode configuration.

The upper limit is estimated by the maximum voltage swing at the input terminal and is $\mathrm{C}_{\text {tot }}$ $\mathrm{x}$ Vswing $/ \mathrm{q}=0.4 \mathrm{pF} \times 0.1 \mathrm{~V} / 1.6 \times 10^{-19} \mathrm{Coul}=250,000$ electrons. Therefore dynamic range is $\sim 48 \mathrm{~dB}$.

\subsection{Discussion}

A double diode readout scheme was tested with a-Si:H pin diodes produced by PECVD directly on the same glass substrate. The measured current on/off ratio was $\sim 10^{5}$ for pin diodes $2 \mu \mathrm{m}$ thick, and an integration time of $10 \mathrm{msec}$ and a readout time of $5 \mu \mathrm{sec}$ was achieved from the dimensions of the prototype devices. The pixel diode and switching diode area were $1 \times 1 \mathrm{~mm}$ and $100 \times 100 \mu \mathrm{m}$ respectively. The diodes showed an onset of surface breakdown at $\sim 5 \mathrm{~V}$ when the application time of bias was greater than a few msec. This breakdown occurred at the etched surface of the a-Si:H layer passivated by the polyimide films. In order to avoid this breakdown, better design will be required, for 
example, making the top area of the metal contacts smaller than that of a-Si:H layers. The measured reverse current was as good as the best quality pin diodes known but the forward current was a factor of $10^{2} \sim 10^{5}$ lower than the best reported. In order to improve the forward conductance, the characteristics of the p-and n-contact layers (doping level, thickness, etc.), as well as the thickness of the i-layer must be optimally determined.

A prototype poly-Si TFT CMOS amplifier was designed and tested. It consisted of a charge-sensitive gain stage, a voltage gain stage and a source follower output stage. The open-loop gain $A_{0}$ of the first stage with the cascode was measured to be $\sim 12$ and of the second stage $\sim 10$. The overall gain-bandwidth product was $\sim 400 \mathrm{MHz}$. When the amplifier is connected to a pixel detector of capacitance $0.2 \mathrm{pF}$, it will give a charge-tovoltage gain of $\sim 0.02 \mathrm{mV} /$ electron with a pulse rise time less than $100 \mathrm{nsec}$ and a dynamic range of $48 \mathrm{~dB}$. From the measurement of the noise-power-spectrum of TFTs, $1 / \mathrm{f}$ noise was found to be dominant noise source up to $10 \mathrm{MHz}$ when it was extrapolated to the point of intersection with the thermal noise. An equivalent noise charge of the front-end nchannel poly-Si TFT was estimated, based on the measured $1 / \mathrm{f}$ noise and the transconductance at the operation bias point, to be $\sim 1000$ electrons rms at a shaping time of $1 \mu \mathrm{sec}$ for $\mathrm{CR}-\mathrm{RC}$ filters. The $1 / \mathrm{f}$ noise can be reduced by decreasing the density of the interface states between silicon and gate oxide.

For large-area integrated electronic devices, the minimum feature size is usually limited by the thermal expansion of the substrate material at the maximum process temperature.[16] For a glass substrate in the low temperature poly-Si technique, the maximum temperature is about $650^{\circ} \mathrm{C}$ and the minimum feature size is about $5 \mu \mathrm{m}$. If quartz is used as a substrate, the minimum feature size can be reduced to $3 \mu \mathrm{m}$ or less because of the superior thermal property of the quartz. The smaller the minimum feature size, the larger gain that can be achieved. Amorphous and poly-silicon TFTs are relatively new devices but of such importance for applications in large-area imaging and consequently they are the subject of intensive and extensive study worldwide. 


\section{References}

[1] R. A. Street, S. Nelson, L. Antonuk and V. Perez-Mendez, "Amorphous Silicon Sensor Arrays for Radiating Imaging," Mat. Res. Soc. Symp. Proc., Vol. 192, 441 (1990)

[2] K. Yamamoto, K. Sai, Y. Ohta, H. Mimura and K. Kitamura, "Amorphous Silicon 2-Dimensional Image Sensors," Techical Digest of the 8th Sensor Symp., p. 55 (1989)

[3] G. P. Weckler, "Operation of p-n Junction Photodetectors in a Photon Flux Integration Mode," IEEE J. Solid Circuits, Vol. SC-2, 65 (1967)

[4] W. den Boer, Y. Baron and Z. Yaniv, "Two-terminal Switches for Active-matrix LCDs," Information Display, 10/90. p. 4 (1990)

[5] A. G. Lewis, I-Wei Wu, T. Y. Huang, A. Chiang and R. H. Bruce, "Active Matrix Liquid Crystal Display Design Using Low and High Temperature Processed Polysilicon TFTs," IEDM-90, p. 843 (1990)

[6] S. M. Sze, VLSI Technology, McGraw-Hill, Second Ed., New-York, p. 471 (1988)

[7] R. Gregorian and G. C. Temes, Analog MOS Integrated Circuits for Signal Processing, John Wiley and Sons, New York, p. 128-129 (1986)

[8] W. A. Lane and G. T. Wrixon, "The Design of Thin Film Polysilicon Resistors for Analog IC Applications," IEEE Trans. Electron Devices, ED-36, 738 (1989)

[9] PSPICE User's Manual, MicroSym. Corp., Palo, Alto.

[10] D. A. Hodges and H. G. Jackson, Analysis and Design of Digital Integrated Circuits, McGraw-Hill, New York (1983)

[11] A. G. Lewis, T. Y. Huang, R. H. Bruce, M. Koyanagi, A. Chiang and I-Wei Wu, "Polysilicon Thin Film Transistors for Analogue Circuit Applications," IEDM-88, p. 264 (1988)

[12] Y. H. Byun, M. Shur, M. Hack and K. Lee, "New Analytical Poly Si Thin Film Transistor Model," First Int. Semicond. Dev. Res. Symp. Proc., p. 537-540 (1991)

[13] P. W. Nicholson, Nuclear Electronics, pp. 45, John Wylie and Sons. Inc., (1974) 


\section{Chapter 6 Conclusion}

Hydrogenated amorphous silicon has many potential advantages in making radiation detectors for various applications in medicine and science. This material is highly radiation resistant and it can be deposited on large-area substrates. Position-sensitive radiation detectors can be made out of a 1-d strip or 2-d pixel array of a-Si: $\mathrm{H}$ pin diodes. In the detector array, thin-film-transistors made of deposited a-Si:H or poly-Si on the same substrate can be used for signal readout and for pixel-level amplifier.

For the design of detector-amplifier system with an optimal signal-to-noise ratio, the signal formation in a reverse biased pin diode and noise comoponents of the detector and TFT amplifier system were analyzed in terms of material and operating parameters; the ionized dangling bond density, the drift mobilities and lifetimes of electrons and holes, dimensions and operating biases of detectors and TFTs, and shaping times.

The radiation signals were calculated based on a simple charge collection model and resulted in a good agreement with measurements from sample diodes using various wavelength light sources and $1 \mathrm{MeV}$ beta particles. These sources simulated three cases of charge generation; a case of uniform generation in the bulk i-layer and two cases of surface generation, at the $\mathrm{p}-\mathrm{i}$ and $\mathrm{n}-\mathrm{i}$ interfaces respectively. Thin photodiode coupled to a CsI(Tl) scintillating layer as well as thick detector layers with multi-buried p-layers can enhance the signal size from the same radiation.

Noise of a radiation detector-amplifier sysicm was calculated in terms of input equivalent noise charge, in order to compare directly with the detector signal. The spectral intensities of (a) shot noise and (b) $1 / \mathrm{f}$ noise from a reverse biased a-Si:H pin diode, and (c) thermal noise and (d) 1/f noise of the front-end TFT of a charge-sensitive preamplifier were formulated and were convoluted with a transfer function of a CR-(RC) ${ }^{n}$ shaping amplifier. The reverse current of an a-Si:H pin diode was modelled by the bulk generation of electron-hole pairs with the Poole-Frenkel effect (field-enhanced thermal emission) and 
was used to calculated the spectral intensity of $1 / \mathrm{f}$ noise of a detector diode, which agreed well to measurements. Noise produced in TFTs was analyzed as a sum of thermal noise and 1/f noise. Measurements of noise spectral power in a-Si:H and poly-Si TFTs showed that $1 / \mathrm{f}$ noise is the dominant component up to $\sim \mathrm{MHz}$ range for both cases.

Optimization of signal-to-noise ratio can be done by choosing a proper combination of operating parameters, for example, dimensions of detectors and front-end TFTs and a proper shaping filter circuit. Time variant filter such as a gated-integrator with double correlation sampling can avoid switching transients of readout TFTs and can reduce $1 / \mathrm{f}$ noise from the front-end TFTs of preamplifier.

Signal readout from a-Si:H pixel detectors using transistor- or diocie-switching method was reviewed. Double-diode switching has the advantage of cancelling the switching transients and controlling the bias level of the detector diodes. Prototype tests of double-diode readout demonstrated that the signal charges can be stored in the detector up to a few msec and can be readout within $\sim \mu \mathrm{sec}$. This time-scale ratio will limit the maximum number of pixels for a given scanning cycle. The ratio, which is directly proportional to the diode on/off current ratio, will improve with improvement in diode quality.

For pixel-level amplification of radiation signals, a prototype charge-sensitive polySi TFT CMOS amplifier was designed, fabricated and tested. It consisted of a chargesensitive-preamplification stage, additional gain stage and an output stage. Measurements showed that the overall gain-bandwidth product was $\sim 400 \mathrm{MHz}$. When the amplifier is connected to a pixel detector of capacitance $0.2 \mathrm{pF}$, it would give a charge-to-voltage gain of $\sim 0.02 \mathrm{mV} /$ electron with a pulse rise time less than $100 \mathrm{nsec}$. An equivalent noise charge of the front-end $n$-channel poly-Si TFT was $\sim 1000$ electrons at a $1 \mu \mathrm{sec}$ shaping time for a simple CR-RC filter. The estimated dynamic range is $\sim 48 \mathrm{~dB}$.

The simple analytical expressions of signal and noise of a-Si:H pin detectors and a poly-Si TFT amplifier system derived here can be used quickly and conveniently when designing an a-Si:H radiation detector system such as a pixel detector. 


\section{Appendix A. A Program for Charge Collection and Signal Formation}

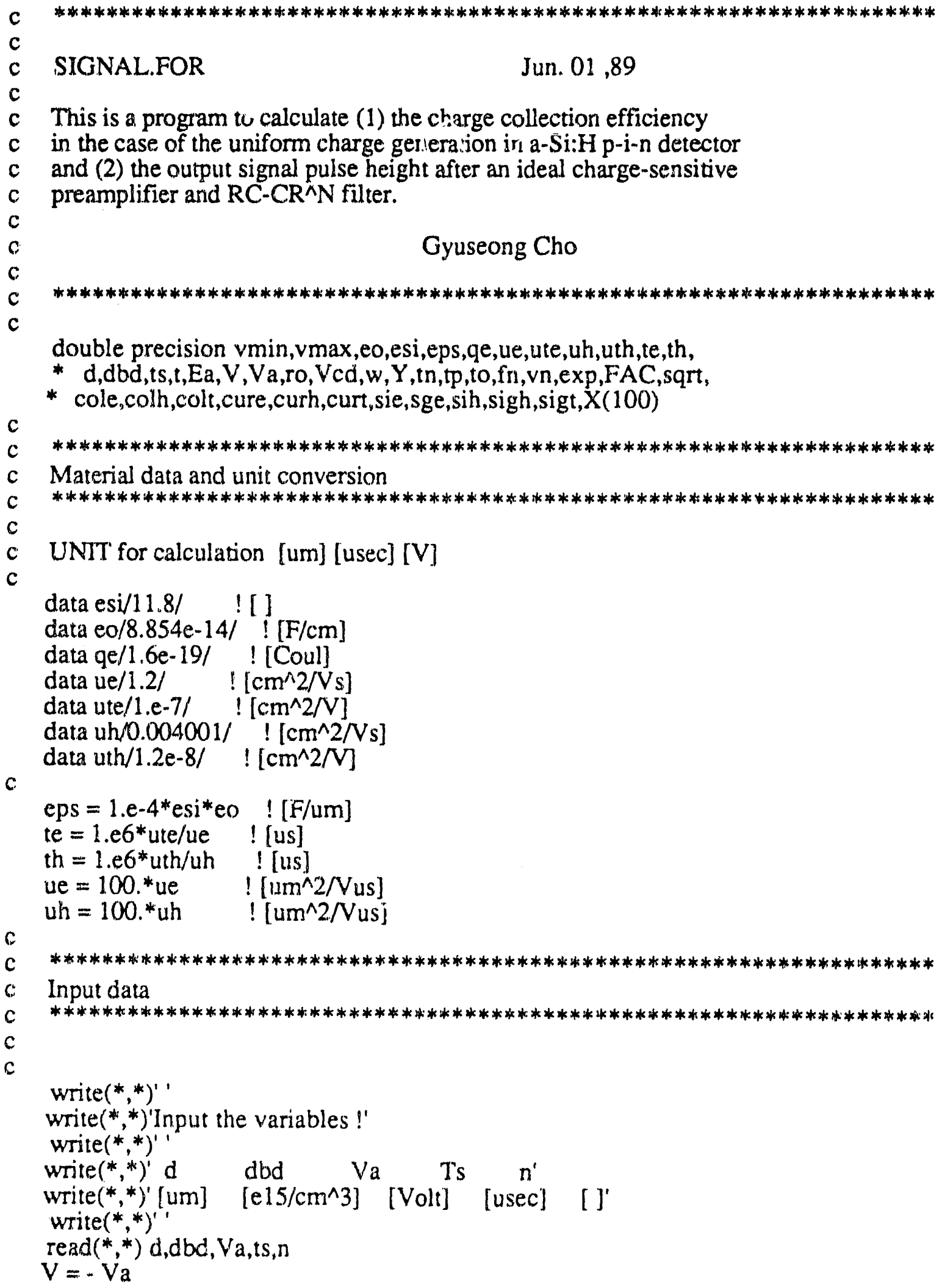




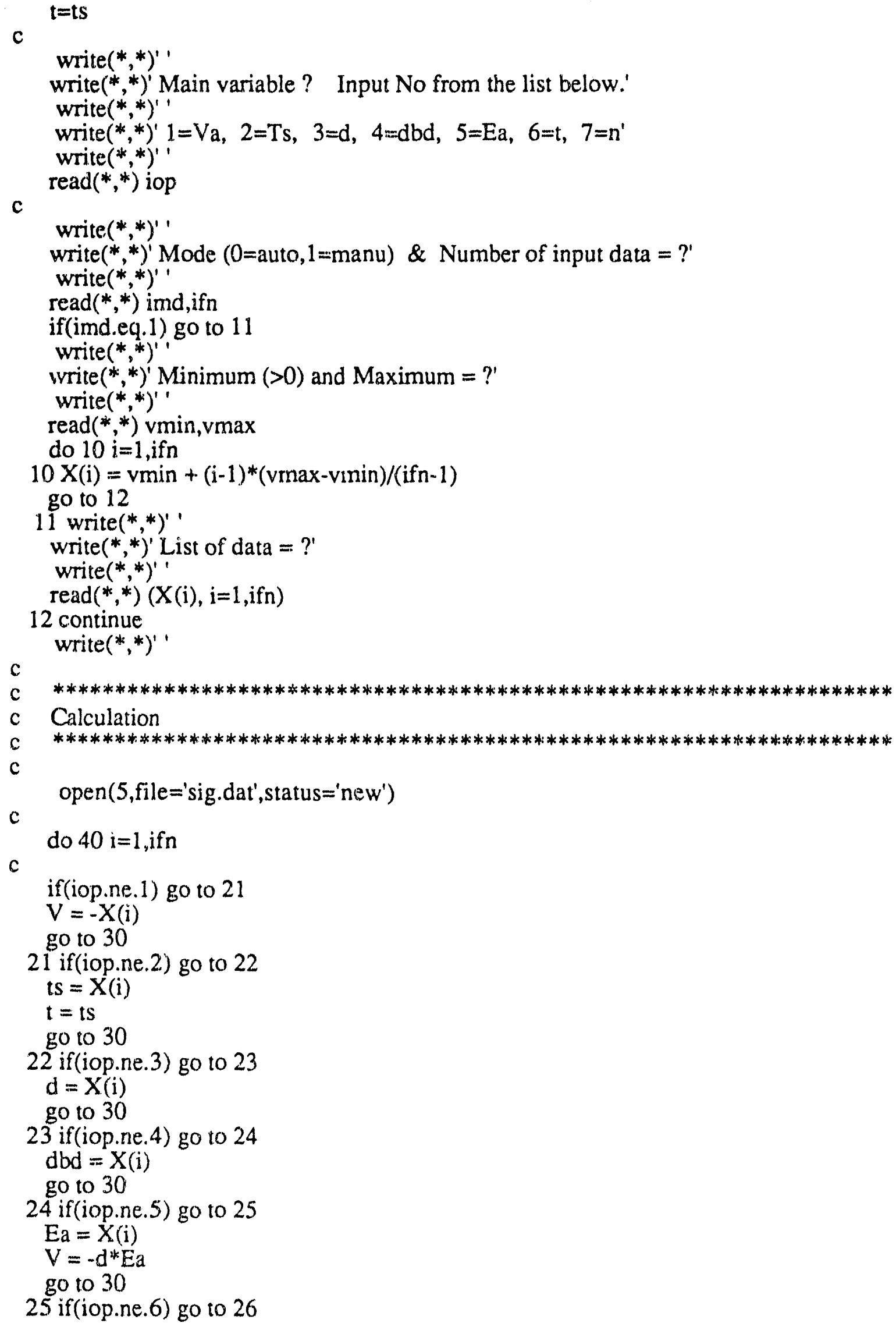


$t=X(i)$

go to 30

26 if(iop.ne.7) go to 27

$\mathrm{N}=\mathrm{X}(\mathrm{i})$

go to 30

27 write $(*, *)^{\prime}$ Failure due to wrong option

c

stop

30 continue

ro $=1000 .^{*} \mathrm{dbd} !\left[\right.$ [ $\left./ \mathrm{um}^{\wedge} 3\right]$

$\mathrm{Vcd}=-\mathrm{qe} * \mathrm{ro}^{*} \mathrm{~d}^{* * 2} /(2 *$ eps $)$ ! full depletion voltage

$\mathrm{w}=\operatorname{sqrt}\left(-2 .^{*}\right.$ eps $* \mathrm{~V} /($ qe $*$ ro $\left.)\right)$

$Y=w / d \quad$ !depletion parameter

tn $=$ eps $/($ ue $*$ qe * ro $)$

tp $=$ eps $/$ ( uh* qe * ro )

to $=\mathrm{ts} / \mathrm{N} \quad !$ Real device $\mathrm{RC}$ time [us] to get peak at ts

$F_{n}=(N / \exp (1)) * *. N / F A C(N)$ ! Voltage output from test pulse

c

$\mathrm{Vn}=\exp (-\mathrm{t} / \mathrm{to}) /\left(\mathrm{to}^{* *} \mathrm{~N} * \mathrm{Fn}\right)$ ! normalizing const

call COLL(t,tn,te,Y,cole,cure)

call COLL(t,ip,th, Y,colh,curh)

colt $=$ cole + colh

cold $=$ colt $* d$

curt $=$ cure + curh $\quad !$ induced current shape

call SIGNAL(t,tn,te,to, $Y, N$, sie $)$

sige $=V n^{*}$ sie

call SIGNAL(t,tp,th,to,Y,N,sih)

$\operatorname{sigh}=V_{n} * \operatorname{sih}$

c

sigt $=$ sige + sigh

write $(*, 101) X(i)$, cole,colh,colt,cold,sige,sigh,sigt

write $(5,101) \mathrm{X}(\mathrm{i})$, cole,colh,colt,cold,sige,sigh,sigt

101 format $(1 \times, f 7.3,7(1 \times, f 7.4))$

c

40 continue

write $(*, *)^{\prime \prime}$

c

stop

end

c

c

c

Subroutine COLL(t,tr,tl,Y,col,cur)

c

c

double precision $t, t r, t l, y, c o l, c u r, a, b, \exp , \log , t t, t m$

$A=(t+\mathrm{t}) /(\mathrm{tr} * \mathrm{t} \mathrm{l})$

$B=(t r-t \mathrm{l}) /(\mathrm{tr} * \mathrm{tl})$

If (Y.gt.1.) go to 10

$\mathrm{Col}=(1 . / 2 .)^{*} \mathrm{Y}^{* * 2} *(1 .-\exp (-\mathrm{A} * t)) /(\mathrm{A} * \mathrm{tr})$

Cur $=(1 . / 2 .)^{*} Y^{* * 2} 2 * \exp \left(-A^{*} t\right) / t r$

go to 20

10 continue

$\mathfrak{t}=\mathrm{tr}^{*} \log \left(\left(\mathrm{Y}^{* * 2}+1.\right) /\left(\mathrm{Y}^{* * 2}-1.\right)\right)$ 


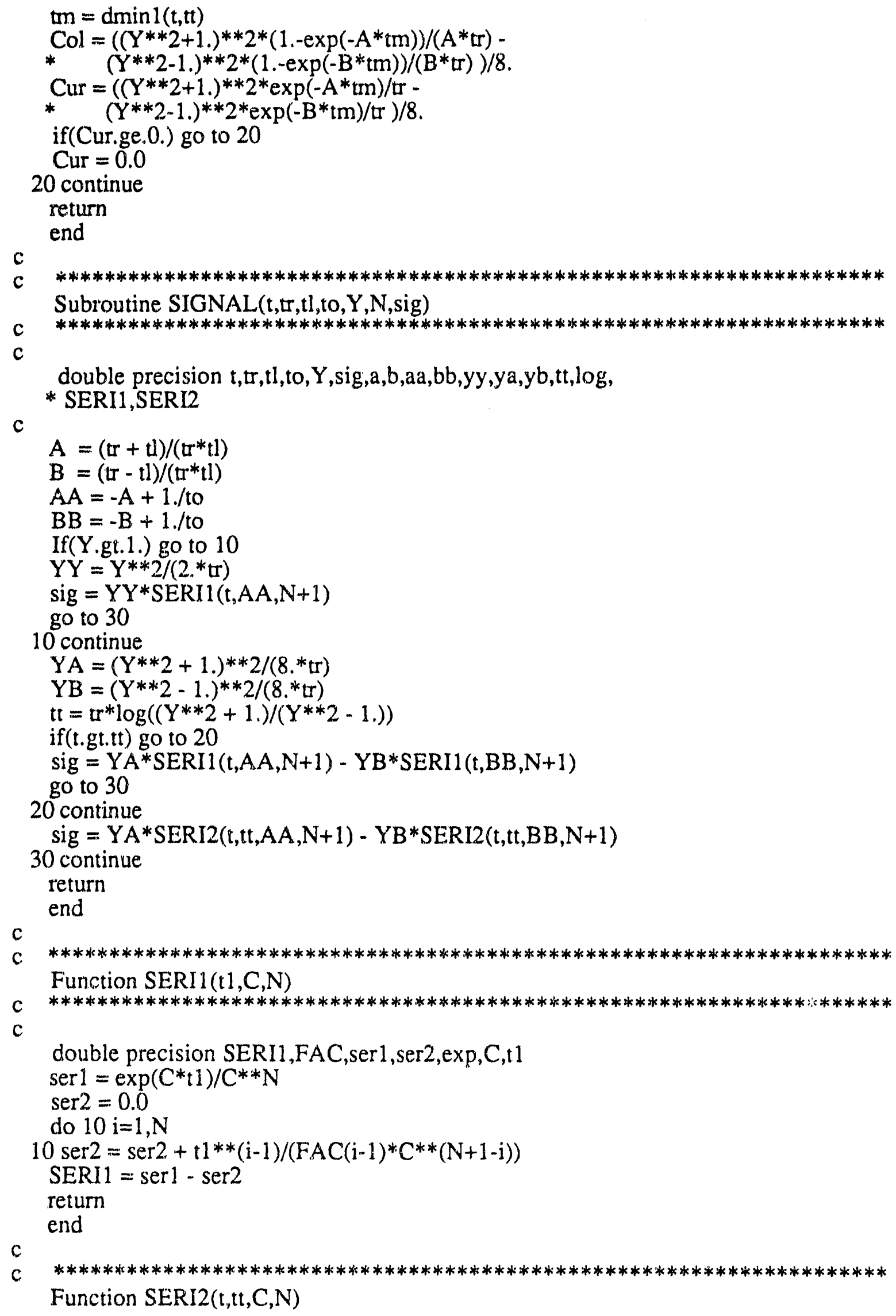


double precision FAC,ser1,ser2,SERI1,SERI2,t,tt,c serl = SERI1 (tt,C,N)

$\operatorname{ser} 2=0.0$

do $10 \mathrm{i}=1, \mathrm{~N}-1$

$10 \operatorname{ser} 2=\operatorname{ser} 2+\operatorname{SERI} 1(\mathrm{tt}, \mathrm{C}, \mathrm{i}) *(\mathrm{t}-\mathrm{tt}) * *(\mathrm{~N}-\mathrm{i}) / \mathrm{FAC}(\mathrm{N}-\mathrm{i})$

SERI2 $=$ ser $1+\operatorname{ser} 2$

return

end

c

c

Function FAC(K)

$c$

$* * * * * * * * * * * * * * * * * * * * * * * * * * * * * * * * * * * * * * * * * * * * * * * * * * * * * * * * * * * * * * * * * * * * * *$

C

double precision $\mathrm{fac}$

$\mathrm{FAC}=1$.

if(K.eq.0) go to 20

do $10 \mathrm{j}=1, \mathrm{~K}$

$\mathrm{FAC}=\mathrm{FAC} * \mathrm{i}$

10 continue

20 continue

return

end 


\section{Appendix B. Charge Collection Efficiency in a Multi-Buried-Layer Detector}

\section{B. 1 Detector Structure and Symbols}

A schematic diagram of the structure of a multi-buried-layer detector (MBLD) is shown in Fig.B.1. Heavily doped $\mathrm{p}^{+}$- and $\mathrm{n}^{+}$-layer work as blocking layers of the injection of electrons and holes from contacting metals to the interaction region respectively. The main interaction region consists of equally spaced $\mathrm{N}+1 \mathrm{i}$-layers and $\mathrm{N}$ slightly doped thin p-layers in sandwich structure. Let's define some symbols for the convenience in deriving the charge collection efficiency.

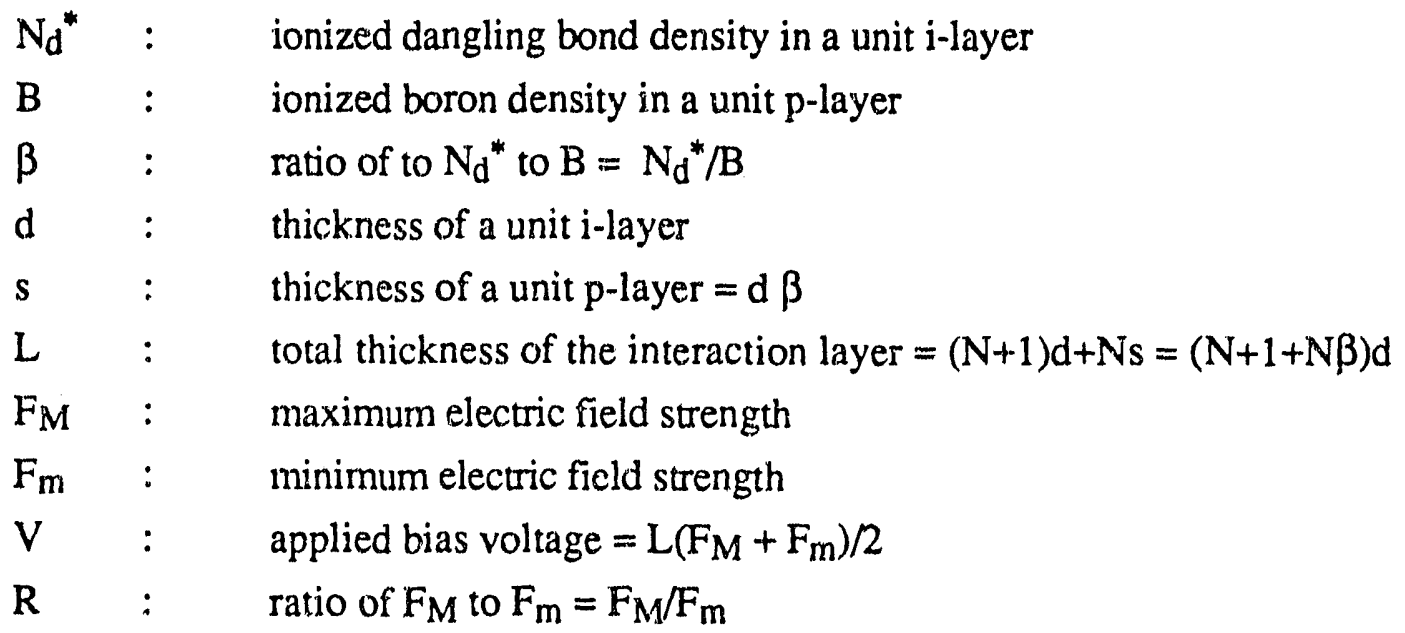

The relation between $F_{M}$ and $F_{m}$ is

$F_{M}=F_{m}+\frac{q N_{d}^{*}}{\varepsilon_{o} \varepsilon_{a s i}} \times d \equiv F_{m}+\delta d \equiv R F_{m}$

Electric Field, F

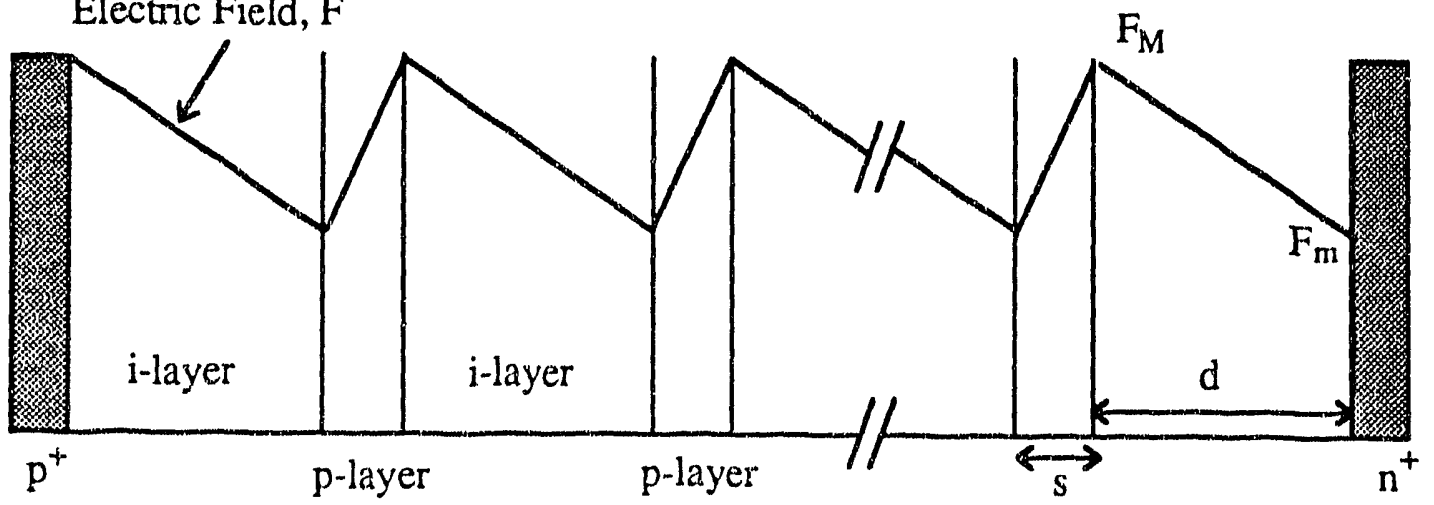

Fig. B.1 A schematic diagram of a multi-buried layer detector. 


\section{B.2 Analysis in a Unit i-layer}

(1) Collection of electrons uniformly generated in a unit i-layer

In a unit $i$-layer of thickness $d$, the electric field distribution $F(x)$ is

$$
F(x)=\frac{F_{m}-F_{M}}{d} x+F_{M}=-\delta x+F_{M}
$$

therefore the drift velocity as a function of position $\mathrm{x}$ is

$$
v(x)=\mu_{i} F(x)=-\mu_{i} \delta x+\mu_{i} F_{M}
$$

where $\mu_{\mathrm{i}}$ is the electron drift mobility in the $\mathrm{i}$-layer.

The velocity of electrons at a time $t$, generated at $x=x_{0}$ at a time $t=0$, is obtained from the above equation using the definition of $v(x)=d x / d t=v\left(x_{0}, x\right)=v\left(x_{0}, t\right)$

$$
v\left(x_{0}, \mathfrak{t}\right)=\left(-\mu_{i} \delta x_{0}+\mu_{i} F_{M}\right) \exp \left(-\mu_{i} \delta \mathfrak{t}\right)
$$

and also the transit time of the electrons from $x=x_{0}$ to $x=d$ is

$$
T_{0}=\int_{x_{0}}^{d} \frac{d x}{v\left(x_{0}, x\right)}=\frac{1}{-\mu_{i} \delta} \log \left(\frac{F_{m}}{-\delta x_{0}+F_{M}}\right) \equiv \frac{\log \left(R_{0}\right)}{\mu_{i} \delta}
$$

where $R_{0}$ is defined as

$$
R_{0} \equiv \frac{-\delta x_{0}+F_{M}}{F_{m}}
$$

The transit time $T$ of electrons from $x=0$ to $x=d$ is

$$
T=T_{0}\left(x_{0}=0\right)=\frac{1}{-\mu_{i} \delta} \log \left(\frac{F_{m}}{F_{M}}\right)=\frac{\log (R)}{\mu_{i} \delta}
$$

If $\tau_{i}$ is the lifetime of electrons in the $i$-layer, the survival probability $\alpha$ of electrons for a time $t$ after their generation is

$$
\alpha(t)=\exp \left(-t / \tau_{i}\right)
$$

then the effective travel distance of electrons generated at $x=x_{0}$ during transit time $T_{0}$ is

$$
\begin{aligned}
S\left(x_{0}\right) & =\int_{0}^{T_{0}} \alpha(t) v\left(x_{0}, t\right) d t \\
& =\frac{-\delta x_{0}+F_{M}}{-\delta-\frac{1}{\mu_{i} \tau_{i}}}\left\{\exp \left[\left(-\delta \mu_{i}-\frac{1}{\tau_{i}}\right) T_{0}\right]-1\right\}
\end{aligned}
$$




$$
\begin{aligned}
& =\frac{-\delta x_{0}+F_{M}}{-\delta-\frac{1}{\mu_{i} \tau_{i}}}\left\{R_{o}^{\left(-1-1 / \delta \mu_{i} \tau_{i}\right)}-1\right\} \\
& =\frac{F_{m}}{-\delta-\frac{1}{\mu_{i} \tau_{i}}}\left\{R_{o}^{\left(-1 / \delta \mu_{i} \tau_{i}\right)}-R_{o}\right\} \\
& =\frac{F_{m}}{-\delta\left(1+\xi_{i}\right)}\left\{R_{o}^{\left(-\xi_{i}\right)}-R_{o}\right\}
\end{aligned}
$$

where $\xi_{\mathrm{i}}$ is defined as $\xi_{\mathrm{i}}=1 /\left(\delta \mu_{\mathrm{i}} \tau_{\mathrm{i}}\right)$.

The contribution of charge collection due to the collection of electrons uniformly generated in the unit i-layer of thickness $d$ is

$$
\begin{aligned}
\chi_{i} & =\int_{0}^{d} S\left(x_{0}\right) d x_{0}=\frac{-F_{m}}{\delta} \int_{R}^{1} S\left(R_{0}\right) d R_{0} \\
& =\left(\frac{F_{m}}{\delta}\right)^{2} \frac{1}{\left(1+\xi_{i}\right)}\left\{\frac{1-R^{\left(1-\xi_{i}\right)}}{1-\xi_{i}}+\frac{R^{2}-1}{2}\right\} \\
& =d^{2}\left(\frac{1}{R-1}\right)^{2}\left\{\frac{1-R\left(1-\xi_{i}\right)}{1-\xi_{i}^{2}}+\frac{R^{2}-1}{2\left(1+\xi_{i}\right)}\right\}
\end{aligned}
$$

(2) Ratio of the escaping electrons to the generated electrons in the i-layer

$$
\begin{aligned}
Z_{i} & =\int_{0}^{d} \varepsilon\left(T_{0}\right) d x_{0}=\int_{0}^{d} \exp \left(\frac{-T_{0}}{\tau_{i}}\right) d x_{0} \\
& =\frac{-F_{m}}{\delta} \int_{R}^{1} R_{0}^{\left(-1 / \delta \mu_{i} \tau_{i}\right)} d R_{0}=\frac{F_{m}}{\delta} \times \frac{R^{\left(1-\xi_{i}\right)}-1}{1-\xi_{i}} \\
& =\frac{d}{R-1} \times \frac{R^{\left(1-\xi_{i}\right)}-1}{1-\xi_{i}}
\end{aligned}
$$

(3) Escaping probability of electrons from $x=0$ to $x=d$ is

$$
P_{i}=\varepsilon(T)=\exp \left(\frac{-T}{\tau_{i}}\right)=R \cdot \xi_{i}
$$

(4) Effective travel distance of electrons from $x=0$ to $x=d$ is

$$
S_{i}=S\left(x_{0}=0\right)=\frac{d}{(1-R)\left(1+\xi_{i}\right)}\left\{R\left(-\xi_{i}\right)-R\right\}
$$




\section{B.3 Analysis in a Unit Buried p-layer}

The analysis of charge collection and transit in a unit p-layer can be done by simply

replacing $d, R, \xi_{i}$, with $s, 1 / R, \xi_{p}\left(\xi_{p}=-\beta /\left(\delta \mu_{p} \tau_{p}\right)\right)$.

(1) Collection of electrons uniformly generated in a unit buried p-layer

$$
\left.\chi_{p}=s^{2}\left(\frac{R}{R-1}\right)^{2} \mid \frac{\left.1-R^{\left(1-\xi_{p}\right.}\right)}{1-\xi_{p}^{2}}+\frac{R^{-2}-1}{2\left(1+\xi_{p}\right)}\right\}
$$

(2) Ratio of the escaping electrons to the generated electrons in the p-layer

$$
Z_{p}=\frac{s R}{R-1} \times \frac{1-R^{\left(\xi_{p}-1\right)}}{1-\xi_{p}}
$$

(3) Escaping probability of electrons from $x=0$ to $x=s$ is

$$
P_{p}=R \xi_{p}
$$

(4) Effective travel distance of electrons from $x=0$ to $x=s$ is

$$
S_{p}=S\left(x_{0}=0\right)=\frac{S R}{(R-1)\left(1+\xi_{p}\right)}\left\{R^{\left(\xi_{p}\right)}-R^{-1}\right\}
$$

\section{B.4 Electron Collection Efficiency in the Total Interaction Region}

The collection efficiency of electrons in the total interaction region is the sum of collection efficiencies from every $i$ - and p-layer.

(1) Collected charge due to electrons generated in the \#1 $\mathrm{i}$-layer is

$\chi_{\mathrm{i}-\mathrm{i}}=$ Contribution due to the transit in \#1 $\mathrm{i}$-layer

+ Contribution due to the transit in \#1 p-layer of survived electrons from $\mathrm{t} \# 1 \mathrm{i}$-layer

+ Contribution due to the transit in \#2 $\mathrm{i}$-layer of survived electrons from \#1 p-layer

$+\ldots . .$.

+ Contribution due to the transit in $\# \mathrm{~N}$ p-layer of survived electrons from $\# \mathrm{~N}$ i-layer

+ Contribution due to the transit in $\# \mathrm{~N}+1 \mathrm{i}$-layer of survived electrons from \#N i-layer

$$
\begin{aligned}
& =\chi_{i}+Z_{i} S_{p}+Z_{i} P_{p} S_{i}+\cdots+Z_{i} P_{p}^{N-1} P_{i}^{N-1} S_{p}+Z_{i} P_{p}^{N} P_{i}^{N-1} S_{i} \\
& =\chi_{i}+Z_{i} \sum_{i=0}^{N-1}\left(P_{i} P_{p}\right)^{i} \times S_{p}+Z_{i} P_{p} \sum_{i=0}^{N-1}\left(P_{i} P_{p}\right)^{i} \times S_{i} \\
& =\chi_{i}+Z_{i}\left(S_{p}+P_{p} S_{i}\right) \times \sum_{i=0}^{N-1}\left(P_{i} P_{p}\right)^{i}
\end{aligned}
$$


(2) Collected charge due to electrons generated in the \#1 p-layer is

$$
\chi_{1-p}=\chi_{p}+Z_{p} S_{i} \times \sum_{i=0}^{N-1}\left(P_{i} P_{p}\right)^{i}+Z_{p} P_{i} S_{p} \sum_{i=0}^{N-2 \geq 0}\left(P_{i} P_{p}\right)^{i}
$$

(3) Total collected charge due to all electrons in the interaction region

$$
\begin{aligned}
\chi_{\text {Lot }}= & \chi_{1-i}+\chi_{1-p}+\chi_{2-i}+\cdots+\chi_{N-p}+\chi_{N+1-i} \\
= & \sum_{K=1}^{N+1} \chi_{K-i}+\sum_{K=1}^{N} \chi_{K-p} \\
= & \sum_{K=1}^{N+1}\left[\chi_{i}+Z_{i}\left(S_{p}+P_{p} S_{i}\right) \times \sum_{i=0}^{K-1}\left(P_{i} P_{p}\right)^{i}\right] \\
& +\sum_{K=1}^{N}\left[\chi_{p}+Z_{p} S_{i} \times \sum_{i=0}^{N-K}\left(P_{i} P_{p}\right)^{i}+Z_{p} P_{i} S_{p} \sum_{i=0}^{N-1-K \geq 0}\left(P_{i} P_{p}\right)^{j}\right] \\
= & (N+1) \chi_{i}+Z_{i}\left(S_{p}+P_{p} S_{i}\right)\left[\frac{N+1}{1-P_{i} P_{p}}-\frac{1-\left(P_{i} P_{p}\right)^{N+1}}{\left(1-P_{i} P_{p}\right)^{2}}\right] \\
& +N \chi_{p}+\frac{N Z_{p}\left(S_{i}+P_{i} S_{p}\right)}{1-P_{i} P_{p}}-Z_{p} P_{i}\left(P_{p} S_{i}+S_{p}\right) \frac{1-\left(P_{i} P_{p}\right)^{N}}{\left(1-P_{i} P_{p}\right)^{2}}
\end{aligned}
$$

(4) Normalized electron collection efficiency is

$$
\eta_{\mathrm{e}}=2 \times \frac{\chi_{\text {tot }}}{\mathrm{L}^{2}}
$$

where the factor two is due to the fact that $\eta_{\mathrm{e}}$ is normalized to the complete collection of electrons only. 


\section{Appendix C. A Summary of Thermal and Field-Enhanced Emission Rates}

\section{C.1 Dirac Well}

C.1.1 Thermal emission

1-dimensional thermal emission

$$
\begin{aligned}
e_{T H}= & \omega_{0} \exp \left[-E_{i} / k T\right] \\
& \text { where } E_{i}=E_{c}-E_{f}
\end{aligned}
$$

3-dimensional thermal emission

same as the above

\section{C.1.2 Tunneling}

1-dimensional tunneling

$$
\begin{aligned}
& e_{T U}= \frac{U}{3 \bar{h}}\left(\frac{U}{E_{i}}\right)^{1 / 2} \exp \left\{-\left(\frac{E_{i}}{U}\right)^{3 / 2}\right\} \\
& \text { where } \\
& U \equiv\left(\frac{3 q \bar{h}}{4 \sqrt{2 m_{*}}} \times F\right)^{2 / 3}
\end{aligned}
$$

3-dimensional tunneling

$$
\begin{aligned}
e_{T U}= & \frac{U}{6 \bar{h}}\left(\frac{U}{E_{i}}\right)^{2} \exp \left\{-\left(\frac{E_{i}}{U}\right)^{3 / 2}\right\} \\
& \text { for } E_{i} \gg U
\end{aligned}
$$

C.1.3 Phonon-assisted tunneling

1-dimensional phonon-assisted tunneling

$$
e_{P T}=\int_{\Delta E_{i}}^{E_{i}} e_{T H}(E) \times \exp \left[-\left(\frac{E}{U}\right)^{3 / 2}\right] \frac{d E}{k T}
$$

3-dimensional phonon-assisted tunneling

$$
e_{P T}=\int_{\Delta E_{i}}^{E_{i}} e_{T H}(E) \times\left(\frac{U}{E}\right)^{2 / 3} \times \exp \left[-\left(\frac{E}{U}\right)^{3 / 2}\right] \frac{d E}{k T}
$$




\section{C.2 Coulomb Well}

\section{C.2.1 Poole-Frenkel emission}

1-dimensional Poole-Frenkel emission

$$
\begin{aligned}
e_{\mathrm{PF}}= & \mathrm{e}_{\mathrm{TH}} \exp [\gamma] \\
& \text { where } \gamma=\Delta \mathrm{E} / \mathrm{kT}=\beta_{\mathrm{PF}} \sqrt{\mathrm{F}} / \mathrm{kT}
\end{aligned}
$$

3-dimensional Poole-Frenkel effect

Hartke model ; neglect the barrier increase in the opposite direction

$$
e_{\mathrm{PF}}=\mathrm{e}_{\mathrm{TH}}\left\{\gamma^{-2}[(\gamma-1) \times \exp (\gamma)+1]+0.5\right\}
$$

Hill model ; include the barrier increase in the opposite direction

$$
e_{P F}=e_{T H}\left\{2 \cosh (\gamma)-\frac{\sinh (\gamma)}{\gamma}\right\}
$$

\section{C.2.2 Tunneling}

1-dimensional tunneling

$$
\text { erU }=\frac{U}{3 \bar{h}}\left(\frac{U}{E_{i}}\right)^{1 / 2} \exp \left\{-\left(\frac{E_{i}}{U}\right)^{3 / 2}\left[1-\left(\frac{\Delta E}{E_{i}}\right)^{5 / 3}\right]\right\}
$$

3-dimensional tunneling

$$
e_{T U}=\frac{U}{6 \bar{h}}\left(\frac{U}{E_{i}}\right)^{2} \exp \left\{-\left(\frac{E_{i}}{U}\right)^{3 / 2}\left[1-\left(\frac{\Delta E}{E_{i}}\right)^{5 / 3}\right]\right\}
$$

\section{C.2.3 Phonon-assisted tunneling}

1-dimensional phonon-assisted tunneling

$$
e_{P T}=\int_{\Delta E_{i}}^{E_{i}} e_{T H}(E) \times \exp \left\{-\left(\frac{E}{U}\right)^{3 / 2}\left[1-\left(\frac{\Delta E}{E}\right)^{5 / 3}\right]\right\} \frac{d E}{k T}
$$

3-dimensional phonon-assisted tunneling

$$
e_{P T}=\int_{\Delta E_{i}}^{E_{i}} e_{T H}(E) \times\left(\frac{U}{E}\right)^{2 / 3} \times \exp \left\{-\left(\frac{E}{U}\right)^{3 / 2}\left[1-\left(\frac{\Delta E}{E}\right)^{5 / 3}\right]\right\} \frac{d E}{k T}
$$




\section{Appendix D. Derivations of Noise Power Spectra}

\section{D.1 Definition of Spectral Intensity}

Let $X(t)$ is a stationary random process for $0 \leq t \leq T$, using a Fourier expansion, $X(t)$ can be rewritten by

$$
X(t)=\sum_{n=-\infty}^{\infty} a_{n} \exp \left(j \omega_{n} t\right)
$$

where $\omega_{n}=2 \pi n / T$ and $n=0, \pm 1, \pm 2, \ldots$

The coefficient $a_{n}$ is

$$
a_{n}=\frac{1}{T} \int_{T}^{T} X(t) \exp \left(-j \omega_{n} t\right) d t
$$

The spectral intensity $S_{X}(f)$ is defined as

$$
S_{X}(f) \equiv \lim _{T \rightarrow \infty} 2 T \overline{a_{n} a_{n}^{*}}
$$

where $a_{n}{ }^{*}$ is the complex conjugate of $a_{n}$.

$S_{X}(f)$ also can be written from the Wiener-Khintchine theorem

$$
\begin{aligned}
S_{X}(f) & =2 \int_{-\infty}^{\infty} \overline{X(t) X(t+s)} \cos (\omega s) d s \\
& =4 \int_{0}^{\infty} \overline{X(t) X(t+s)} \cos (\omega s) d s
\end{aligned}
$$

\section{D.2 Shot Noise}

Let $I_{O}$ is the average flow of current through some region for a time $T$, then the average number of charges crossing the region boundary is

$$
N=\frac{I_{0}}{q} T
$$

where $q$ is the electron charge. 
Assuming the Poisson statistics, the fluctuation in $\mathrm{N}$ becomes

$$
\Delta \mathrm{N}=\sqrt{\mathrm{N}}
$$

The charge associated due to the fluctuation is

$$
\Delta Q=q \operatorname{Qi} N=\mathrm{q} \sqrt{\mathrm{N}}
$$

and the fluctuation in the observed current is

$$
\Delta \mathrm{I}_{\mathrm{o}}=\frac{\Delta \mathrm{Q}}{\mathrm{T}}=\frac{\mathrm{q} \sqrt{\mathrm{N}}}{\mathrm{T}}=\sqrt{\frac{\mathrm{qI}}{\mathrm{T}}}
$$

If $\Delta \mathrm{l}(\mathrm{t})$ is a stationary random variable having the average $\Delta \mathrm{I}_{\mathrm{O}}$ over an time interval $\mathrm{T}$ i.e., then the low frequency spectrum is

$$
S_{\Delta I}(0) \equiv \lim _{T \rightarrow \infty} 2 T \overline{\Delta I_{T}^{2}}=\lim _{T \rightarrow \infty} 2 T \Delta I_{0}^{2}=2 q I_{0}
$$

\section{D.3 Generation-Recombination Noise}

Let's consider recombination centers having a decaying characteristic time constant $\tau$. If $\mathrm{N}$ is the average number of electrons in the recombination center at steady state and $\Delta \mathrm{N}$ is the fluctuation of $\mathrm{N}$ at $\mathrm{t}=0$ then $\Delta \mathrm{N}(\mathrm{t})$ at a time $\mathrm{t}$ is

$$
\Delta N(t)=\Delta N \times \exp [-t \tau]
$$

The auto correlation function is

$$
\overline{\Delta N(t) \Delta N(t+s)}=\overline{\Delta N^{2}} \exp (-s / \tau)
$$

Then using the Wiener-Khintchine theorem the spectral intensity is

$$
\begin{aligned}
S_{\Delta N}(f) & =2 \int_{-\infty}^{\infty} \overline{\Delta N(t) \Delta N(t+s)} \cos (\omega s) d s \\
& =4 \overline{\Delta N^{2}} \int_{0}^{\infty} \exp (-s / \tau) \cos (\omega s) d s \\
& =4 \overline{\Delta N^{2}} \frac{\tau}{1+\omega^{2} \tau^{2}}
\end{aligned}
$$




\section{D.4 Thermal Noise in a Resistor}

Consider an RC network with a thermal noise source $V_{R}(t)$ associated with the resistance $R$ as shown in the Fig. D.1 where $R$ is a noiseless resistance. $C$ is an ideal capacitor of which impedance is $1 / j \omega C$. Then the magnitude of $\mathrm{ms}$ voltage across $\mathrm{C}$ is given by

$$
V_{C}^{2}(f)=\frac{V_{R}^{2}(f)}{1+\omega^{2} R^{2} C^{2}}
$$

The total rms voltage across the capacitor is then

$$
V_{\text {ms }}^{2}=\int_{0}^{\infty} V_{C}^{2}(f) d f=\int_{0}^{\infty} \frac{V_{R}^{2}(f)}{1+\omega^{2} R^{2} C^{2}} d f
$$

This integral is only converging when $V_{R}(f)$ is independent of $f$ therefore if we assume that, then

$$
\begin{aligned}
V_{\text {Ims }}^{2} & =V_{R}^{2} \int_{0}^{\infty} \frac{1}{1+\omega^{2} R^{2} C^{2}} d f=\frac{V_{R}^{2}}{2 \pi R C}\left[\tan ^{-1}(\infty)-\tan ^{-1}(0)\right] \\
& =\frac{V_{R}^{2}}{2 \pi R C}\left[\frac{\pi}{2}-0\right]=\frac{V_{R}^{2}}{4 R C}
\end{aligned}
$$

Now consider the total energy associated with a voltage $V$ across a capacitor which is $\mathrm{C}^{2} \mathrm{~V} / 2$. Fluctuations in this energy is calculated from the equipartion of energy given by

$$
\frac{1}{2} \mathrm{CV}_{\mathrm{rms}}^{2}=\frac{1}{2} \mathrm{kT}
$$

therefore

$$
V_{R}^{2}=4 k T R
$$
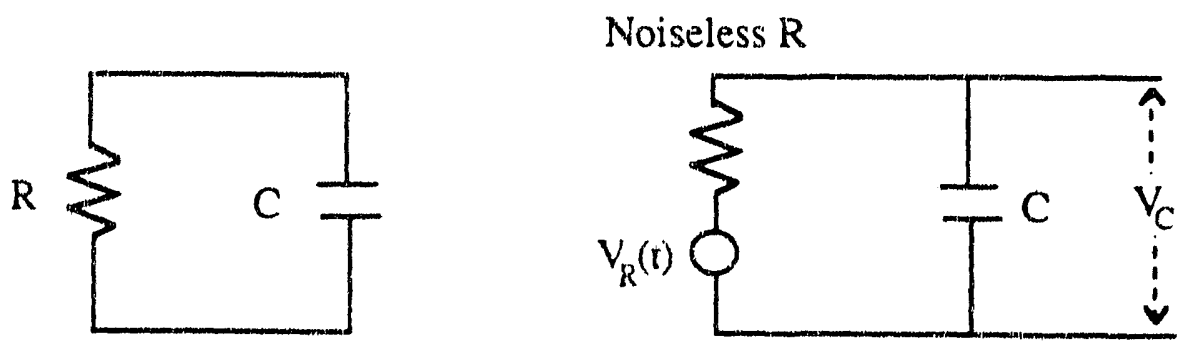

Fig. D.1 RC network and equivalent circuit for a thermal resistive-noise analysis. 


\section{Appendix E. Evaluation of ENC for a CR $(R C)^{n}$ Filter}

\section{E.1 Gain of CR-(RC) $)^{\text {n Filter }}$}

For $C R$ differential circuit, $V_{\text {in }}(t)$ and $V_{\text {out }}(t)$ has a relation

$$
\frac{d V_{\text {out }}(t)}{d t}+\frac{1}{R C} V_{\text {out }}(t)=\frac{d V_{\text {in }}(t)}{d t}
$$

Laplace transform is

$$
s \widetilde{V}_{\text {out }}(s)+a \tilde{V}_{\text {out }}(s)=s \widetilde{V}_{\text {in }}(s)
$$

where $\mathrm{a}=1 / \mathrm{RC}$.

Therefore the transfer function is

$$
G_{C R}(s) \equiv \frac{\widetilde{V}_{\text {our }}(s)}{\widetilde{V}_{\text {in }}(s)}=\frac{s}{s+a}
$$

For $R C$ integral circuit, $V_{\text {in }}(t)$ and $V_{\text {out }}(t)$ has a relation

$$
\frac{d V_{\text {out }}(t)}{d t}+\frac{1}{R C} V_{\text {out }}(t)=\frac{1}{R C} V_{\text {in }}(t)
$$

Laplace transform is

$$
s \widetilde{V}_{\text {out }}(s)+a \widetilde{V}_{\text {out }}(s)=a \widetilde{V}_{\text {in }}(s)
$$

Therefore the transfer function is

$$
G_{C R}(s) \equiv \frac{\widetilde{V}_{\text {oul }}(s)}{\widetilde{V}_{\text {in }}(s)}=\frac{a}{s+a}
$$

The net transfer function of $C R-(R C)^{n}$ filter (one $C R$ and $n$ RC circuit) is

$$
G_{n}(s) \equiv \frac{\widetilde{V}_{\text {our }}(s)}{\widetilde{V}_{\text {in }}(s)}=\frac{s a^{n}}{(s+a)^{n+1}}
$$

Output to a step input is

$$
\tilde{V}_{\text {out }}(s)=G_{n}(s) \times \widetilde{V}_{\text {in }}(s)=G_{n}(s) \times \frac{1}{s}=\frac{a^{n}}{(s+a)^{n+1}}
$$

Therefore output in time domain is 


$$
\mathrm{V}_{\text {out }}(\mathrm{t})=L^{-1}\left(\widetilde{\mathrm{V}}_{\text {out }}(\mathrm{s})\right)=\frac{(\mathrm{a} t)^{\mathrm{n}} \mathrm{e}^{-\mathrm{at}}}{\mathrm{n} !}
$$

The peak of the output pulse is obtained by

$$
\frac{d \mathrm{~V}_{\text {out }}(\mathrm{t})}{\mathrm{dt}}=0 \text { at } \mathrm{t}=\tau
$$

then $\tau=\mathrm{n} / \mathrm{a}=\mathrm{nRC}$ and is called the peaking time or the shaping time.

The peak of the output pulse to a unit step input is a gain by definition and is given by

$$
A_{n} \equiv V_{\text {out }}(\tau)=\frac{(a \tau)^{n} e^{-a \tau}}{n !}=\frac{(n)^{n} e^{-n}}{n !}
$$

\section{E.2 Transfer Function in Frequency Domain}

The transfer function of CR differential circuit in the frequency domain is

$$
\mathrm{G}_{\mathrm{CR}}^{2}(\omega)=\left|\frac{Z_{\mathrm{R}}}{\mid \mathrm{Z}_{\mathrm{tot}}}\right|^{2}=\left|\frac{\mathrm{R}}{\mathrm{R}+\frac{1}{j \omega \mathrm{C}}}\right|^{2}=\frac{\omega^{2}}{\mathrm{a}^{2}+\omega^{2}}
$$

where $\mathrm{Z}$ is the impedance, $\omega=2 \pi f$, and $a=1 / R C$.

The transfer function of $\mathrm{RC}$ integral circuit is

$$
G_{R C}^{2}(\omega)=\left|\frac{Z_{C}}{Z_{t o t}}\right|^{2}=\left|\frac{\frac{1}{j \omega C}}{R+\frac{1}{j \omega C}}\right|^{2}=\frac{a^{2}}{a^{2}+\omega^{2}}
$$

Therefore the net transfer function of $C R-(R C)^{n}$ filter is

$$
G_{n}^{2}(\omega)=\frac{a^{2 n} \omega^{2}}{\left(a^{2}+\omega^{2}\right)^{n+1}}
$$

\section{E.3 Input Equivalent Noise Charge}

If the noise input power has a form

$$
\frac{\left\langle v_{\text {in }}^{2}>\right.}{\Delta \mathrm{f}}=\frac{K_{m}}{\omega^{m}}
$$

where $\mathrm{K}_{\mathrm{m}}$ is a constant and $\mathrm{m}$ is an integer then the noise voltag : output is

$$
\left\langle v_{\text {oul }}^{2}\right\rangle=\int_{0}^{\infty}\left\langle v_{\text {in }}^{2}\right\rangle G_{n}^{2}(\omega) d f=\int_{0}^{\infty} \frac{K_{m}}{\omega^{m}} \times \frac{a^{2 n} \omega^{2}}{\left(a^{2}+\omega^{2}\right)^{n+1}} \frac{d \omega}{2 \pi}
$$


The input equivalent noise charge $N$ with a total input capacitance $C_{\text {tot }}$ of a preamplifier is

$$
\mathrm{N}^{2}=\frac{\mathrm{C}_{\mathrm{lol}}^{2}<\mathrm{v}_{\text {out }}^{2}>}{\mathrm{q}^{2} \mathrm{~A}_{\mathrm{n}}^{2}}
$$

where $q$ is an electronic charge and $A_{n}$ is the gain obtained in the above.

Then

$$
N^{2}=\frac{K_{m} C_{l o t}^{2}}{2 \pi q^{2}} \times \frac{a^{2 n} e^{2 n}(n !)^{2}}{n^{2 n}} \int_{0}^{\infty} \frac{\omega^{2-m}}{\left(a^{2}+\omega^{2}\right)^{n+1}} d \omega
$$

For $m=0$ (thermal noise of TFT and series resistance)

$$
\int_{0}^{\infty} \frac{\omega^{2}}{\left(a^{2}+\omega^{2}\right)^{n+1}} d \omega=\frac{\pi(2 n) !}{(2 n-1) 2^{2 n+1}(n !)^{2} a^{(2 n-1)}}
$$

For $m=1(1 / \mathrm{f}$ noise of TFT $)$

$$
\int_{0}^{\infty} \frac{\omega}{\left(a^{2}+\omega^{2}\right)^{n+1}} d \omega=\frac{1}{2 n a^{(2 n)}}
$$

For $m=2$ (shot noise of detector)

$$
\int_{0}^{\infty} \frac{1}{\left(a^{2}+\omega^{2}\right)^{n+1}} d \omega=\frac{\pi(2 n) !}{22^{2 n}(n !)^{2} a^{(2 n+1)}}
$$

For $m=3(1 / \mathrm{f}$ noise of detector $)$

$$
\int \frac{1}{\omega\left(a^{2}+\omega^{2}\right)^{n+1}} d \omega=\frac{1}{2 a^{(2 n+2)}} \times\left[\sum_{i=1}^{n} \frac{a^{2 i}}{i\left(a^{2}+\omega^{2}\right)^{2}}-\ln \left(\frac{a^{2}+\omega^{2}}{\omega^{2}}\right)\right]
$$

The last term in the bracket goes to $\infty$ when $\omega$ approaches zero. To avoid the divergence, we will use $\omega_{\min }$ instead of zero. Then approximately

$$
\int_{0}^{\infty} \frac{1}{\omega\left(a^{2}+\omega^{2}\right)^{n+1}} d \omega \approx \frac{1}{2 a(2 n+2)} \times\left[2 \ln \left(\frac{a}{\omega_{\min }}\right)-\sum_{i=1}^{n} \frac{1}{i}\right] \approx \frac{1}{a^{(2 n+2)}} \times \ln \left(\frac{a}{\omega_{\min }}\right)
$$

where $\omega$ is found from the relation

$$
\omega_{\min }=2 \pi f_{\min }=\omega_{0} \exp \left[\left(E_{\mathrm{f}}-E_{c}\right) / k T\right]
$$




$$
\approx 10^{12} \exp (-0.9 / 0.025) \approx 2.32 \times 10^{-4}
$$

For a reasonable range of $a=n / \tau$ corresponding to a typical shaping time $\tau$, the summation in the bracket is negligible compared to logarithmic term,

Therefore the input equivalent noise is

$$
\begin{aligned}
& N_{0}^{2}=\frac{K_{0} C_{\text {lot }}^{2}}{2 \pi q^{2}} \times\left(\frac{\pi n(2 n) ! e^{2 n}}{2(2 n-1) 2^{2 n} n^{2 n}}\right) \times \frac{1}{\tau} \\
& N_{1}^{2}=\frac{K_{1} C_{l o t}^{2}}{2 \pi q^{2}} \times\left(\frac{(n !)^{2} e^{2 n}}{2 n n^{2 n}}\right) \\
& N_{2}^{2}=\frac{K_{2} C_{\text {lot }}^{2}}{2 \pi q^{2}} \times\left(\frac{\pi(2 n) ! e^{2 n}}{2 n 2^{2 n} n^{2 n}}\right) \times \tau \\
& N_{3}^{2}=\frac{K_{3} C_{\text {tot }}^{2}}{2 \pi q^{2}} \times\left(\frac{(n !)^{2} e^{2 n}}{n^{2} n^{2 n}} \times \ln \left(\frac{n}{\tau \omega_{\text {min }}}\right)\right) \times \tau^{2}
\end{aligned}
$$

Generally we can write

$$
N_{m, n}^{2}=\frac{K_{m} C_{t o l}^{2}}{2 \pi q^{2}} \times F_{m, n} \times \tau^{m-1}
$$

and the values of $\mathrm{F}_{\mathrm{m}, \mathrm{n}}$ for $\mathrm{CR}-\mathrm{RC}$ and $\mathrm{CR}-(\mathrm{RC})^{4}$ are listed in the table below.

\begin{tabular}{|c||c|c|}
\hline & $n=1$ & $n=4$ \\
\hline$m=0 \quad$ (thermal noise) & $2 \pi \times 0.9236$ & $2 \pi \times 1.023$ \\
\hline$m=1 \quad$ (TFT $1 / \mathrm{f}$ noise) & 3.695 & 3.275 \\
\hline$m=2 \quad$ (shot noise) & $2 \pi \times 0.9236$ & $2 \pi \times 0.4478$ \\
\hline$m=3$ (detector $1 / \mathrm{f}$ noise) & $7.389 \times \ln \left(1 / \tau \omega_{m}\right)$ & $1.637 \times \ln \left(4 / \tau \omega_{m}\right)$ \\
\hline
\end{tabular}




\section{Appendix F. Time Domain nalysis of a Gated-Integrator Filter}

The time domain analysis of step noise and delta noise for a gated-integrator filter will be given here. If unit-amplitude noise steps occur randomly but with an average rate $\mathrm{n}_{\mathrm{S}} / \mathrm{sec}$ then the average number $\mathrm{N}$ of noise steps in $\mathrm{dt}$ is $\mathrm{N}=\mathrm{n}_{\mathrm{S}} \mathrm{dt}$. The mean square fluctuation in $\mathrm{N}$ is also equal to $\mathrm{n}_{\mathrm{S}} \mathrm{dt}$ because of Poisson statistics. Noise step occurring at a time $t$ (measured backward) before a msasuring time has an effect on the base level of the signal at the measuring time, defined as $R(t)$. Then the mean square effect on the signal due to $\mathrm{N}$ noise steps in $\mathrm{dt}$ is equal to:

$$
\mathrm{n}_{\mathrm{s}} \mathrm{R}(\mathrm{t})^{2} \mathrm{dt}
$$

Then all noise steps having occurred before the measuring time are integrated together to find the mean square fluctuations in the noise at the measuring time $T_{m}$.

$$
\text { Step Noise Index }=\left(\frac{1}{A}\right)^{2} \int_{0}^{\infty} R(t)^{2} d t
$$

where $A$ is the output amplitude due to an input step with an unity magnitude.

Similarly a noise index can be defined for delta noise. Delta noise can be considered as a step noise of with a magnitude proportional to $1 / \Delta t$ followed $\Delta t$ later by a negative step function with the same magnitude. Therefore its noise effect will be

$$
\frac{1}{\Delta t} R(t)-\frac{1}{\Delta t} R(t-\Delta t)
$$

For $\Delta t$ approaches 0 , this function is a differential of $R(t)$. Delta noise residual function is $R^{\prime}(t)$.

Delta Noise Index $=\left(\frac{1}{\mathrm{~A}}\right)^{2} \int_{0}^{\infty} \mathrm{R}^{\prime}(\mathrm{t})^{2} \mathrm{dt}$

If a square current input pulse with the height $\mathrm{V}$ and a duration time $\mathrm{T}$ is fed into a gated integrator filter with an integration time $T_{I}$ then the output voltage pulse would be a trapezoidal shape with a rising time $T_{r}=T$ and a flat-top duration $T_{f}=T_{I}-T$ as shown in the figure below. The time at which we measure the signal is called a measuring time $T_{m}$. 
The step-noise residual function $R(t)$ is obtained by a convolution of the the rectangular pulse of width $T$ with another rectangular function of width $T_{I}$. Once $R(t)$ is obtained then $R^{\prime}(t)$ also can be found by a differentiation. The output signal shape, $R(t)$, $R^{\prime}(t), R(t)^{2}$ and $R^{\prime}(t)^{2}$. are shown in the figure below. Now using the noise index equations for step noise and delta noise and assuming $A=1$, both noise indices are

$$
\left\langle\mathrm{N}_{\mathrm{S}}{ }^{2}\right\rangle=\mathrm{T}-\mathrm{T}_{\mathrm{I}} / 3
$$

and

$$
\left\langle N_{\Delta}^{2}\right\rangle=2 / T
$$
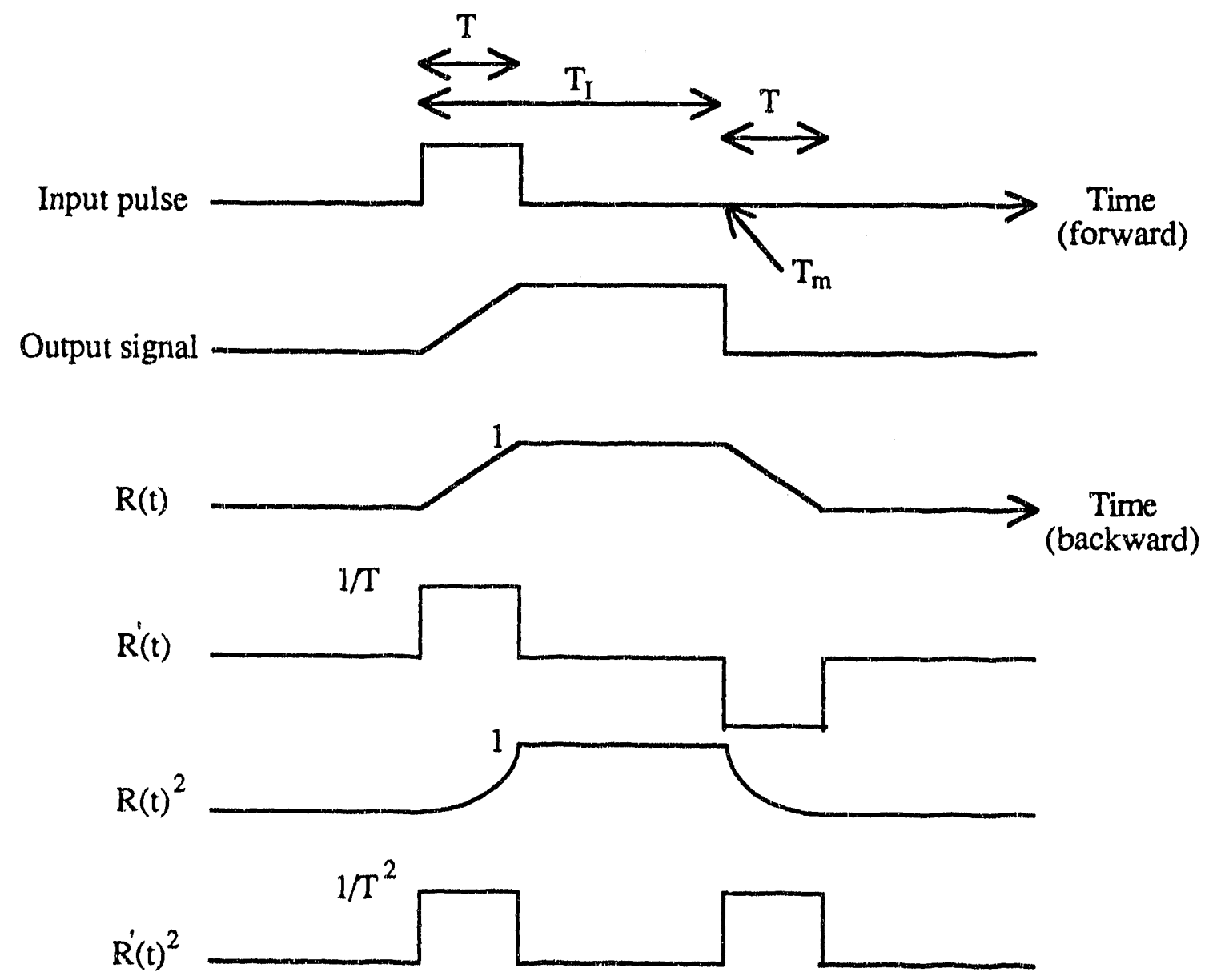

Fig. F.1 Timing diagram of a gated integrator showing input pulse, output signal, $R(t)$, $R^{\prime}(t), R(t)^{2}$ and $R^{\prime}(t)^{2}$. (Ref. F, S. Goulding, "Pulse-Shaping in Low-Noise Nuclear Amplifiers: A Physical Approach to Noise Analysis," Nuc. Instr. Meth., 100, 493 (1972).) 

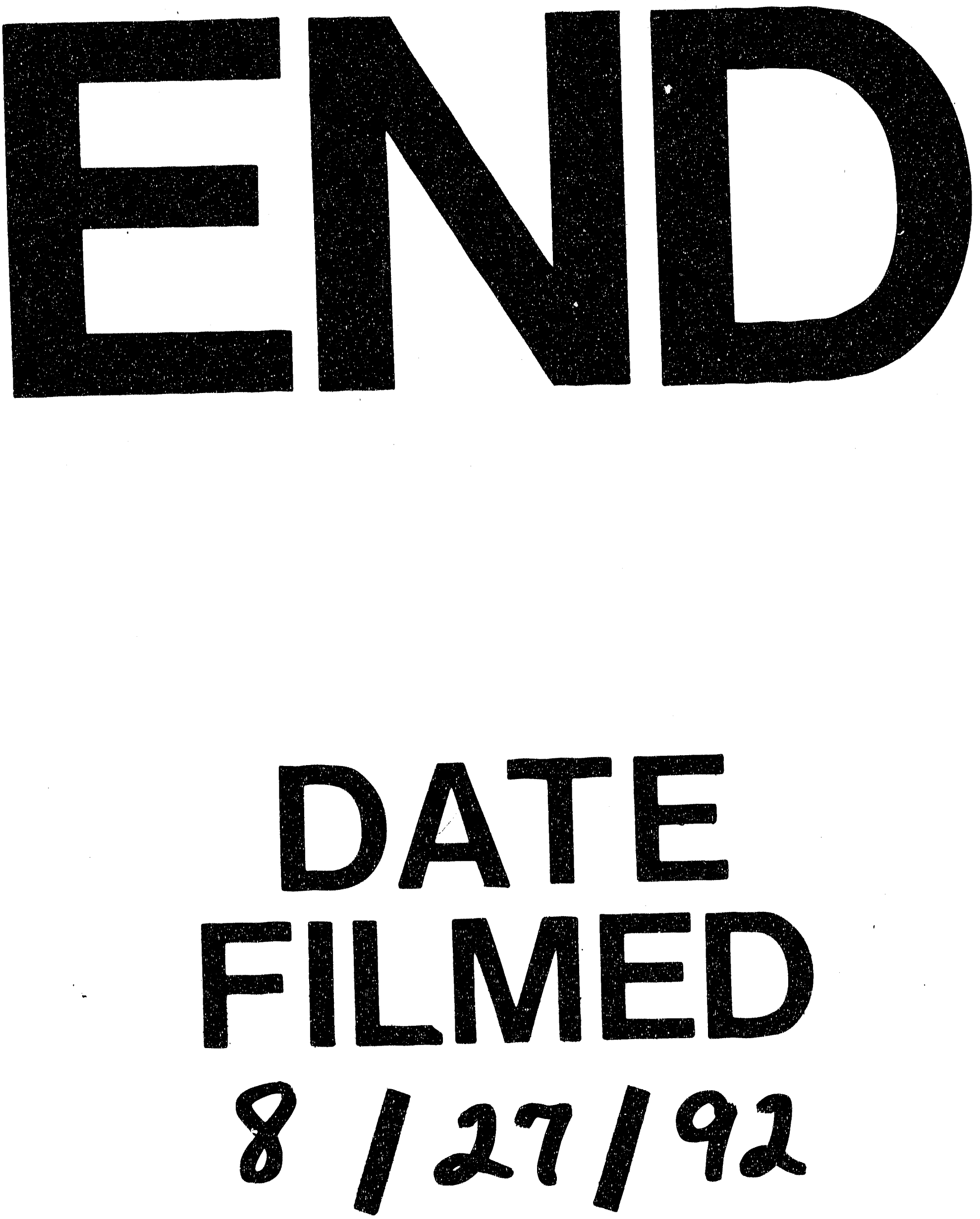

I 
1 